\title{
WHISKERS, BARRELS, AND CORTICAL EFFERENT PATHWAYS IN GAP CROSSING BY RATS
}

\author{
Edward Walter Jenkinson
}

Submitted For The Degree Of Doctor Of Philosiphy

Department Of Anatomy \& Developmental Biology

University College London 
ProQuest Number: 10611117

All rights reserved

INFORMATION TO ALL USERS

The quality of this reproduction is dependent upon the quality of the copy submitted.

In the unlikely event that the author did not send a complete manuscript and there are missing pages, these will be noted. Also, if material had to be removed, a note will indicate the deletion.

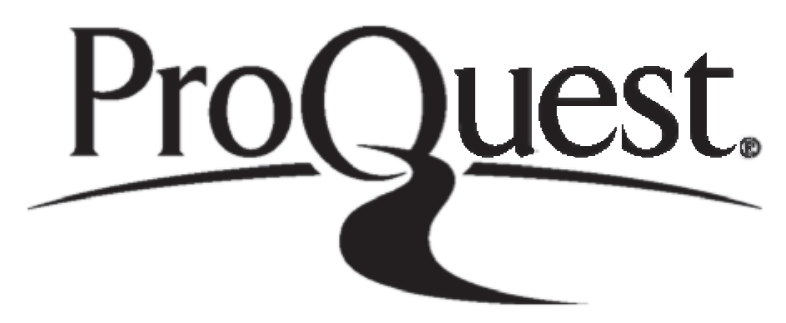

ProQuest 10611117

Published by ProQuest LLC (2017). Copyright of the Dissertation is held by the Author.

All rights reserved.

This work is protected against unauthorized copying under Title 17, United States Code Microform Edition (C) ProQuest LLC.

ProQuest LLC.

789 East Eisenhower Parkway

P.O. Box 1346

Ann Arbor, Ml $48106-1346$ 


\section{Abstract}

\section{WHISKERS, BARRELS, AND CORTICAL EFFERENT PATHWAYS IN GAP CROSSING BY RATS}

Rats can readily be trained to jump a gap of around $30 \mathrm{~cm}$ in the light and $16 \mathrm{~cm}$ in the dark for a food reward. In the light they use vision to estimate the distance to be jumped. In the dark they use their vibrissae at the farthest distances. Bilateral whisker shaving or barrel field lesions reduce the gap crossed in the dark by about $2 \mathrm{~cm}$ (Hutson and Masterton, 1986). Information from the barrel fields reaches motor areas via the cortico-cortical, basal ganglia, or cerebellar pathways. The cells of origin of the pontocerebellar pathway are segregated in layer $\mathrm{Vb}$ of the barrel field (Mercier et al., 1990). Efferent axons of $\mathrm{Vb}$ cells occupy a central position within the basis pedunculi, and terminate on cells in the pontine nuclei (Glickstein et al., 1992). Pontine cells, in turn, project to the cerebellar cortex as mossy fibres. We trained normal rats to cross a gap in the light and in a dark alley that was illuminated with an infra-red source. When the performance was stable we made unilateral lesions in the central region of the basis pedunculi which interrupted connections from the barrel field to the pons whilst leaving cortico-cortical and basal ganglia pathways intact. Whisking was not affected on either side by the lesion and the rats with unilateral peduncle lesions crossed gaps of the same distance as they did pre-operatively. Shaving the whiskers in the side of the face that retains its input to the pontine nuclei reduced the maximal gap jumped in the dark by the same amount as bilateral whisker shaving. Performance in the light was not affected. Re-growth of the shaved whiskers was associated with the recovery of the maximum distance crossed in the dark. In control cases, shaving the whiskers on the other side of the face did not reduce the distance jumped in the dark or in the light. These results suggests that the cerebellum must receive whisker information from the barrel fields from the barrel fields for whisker-guided jumps. 


\section{Acknowledgements}

First and foremost I must thank Professor Mitch Glickstein, my mentor throughout my time at University College, and supervisor of this $\mathrm{PhD}$. Without his guidance, tenacious patience and wisdom it is doubtful this thesis would have ever been completed. Credit must also be given to Ines Hans and Catherine Swales, who are simply the finest colleagues one could wish for. Also, I thank Dan Adams and Kostas Moutoussis for their camaraderie through the many highs and very few lows of undergraduate and postgraduate neuroscience at UCL. Finally, thanks to Professor Ford Ebner and his staff, especially Robert Sachdev, at the Institute For Developmental Neuroscience at Vanderbilt University, Nashville, for allowing me to work in their lab during the completion of this thesis. 


\section{$\underline{\text { Table Of Contents }}$}

\section{INTRODUCTION 10}

What Are Vibrissae? 11

The Mystacial Vibrissae 12

Structure Of The Vibrissae And Follicle 13

Neural Innervation 15

Deep-Vibrissal Nerves 15

The Superficial-Vibrissal Nerve 16

Receptor Types And Distribution 16

The Trigeminal System 21

The Trigeminal Nerve And Ganglion 21

Response Properties In The Trigeminal Ganglion 22

Somatotopy In The Trigeminal Ganglion 23

The Trigeminal-Nuclear Complex 24

The Principle-Sensory Nucleus 25

The Spinal Trigeminal Subnucleus Oralis 26

The Spinal Trigeminal Subnucleus Interpolaris 27

The Spinal Trigeminal Subnucleus Caudalis 29

Somatotopy In The Trigeminal-Nuclear Complex 29

Targets Of The Trigeminal-Nuclear Complex 31

Trigeminal-Nuclear Complex Efferents To The Superior Colliculus 31

Trigeminal-Nuclear complex Efferents To The Cerebellum 33

Trigeminal-Nuclear complex Efferents To The Thalamus 34 
The Thalamo-Cortical Projection 37

Ventroposteromedial Nucleus (Vpm) 37

Posterior Nucleus (PO) 39

Somatosensory Cortex In The Rat And Mouse 44

Cortical Barrels 44

Barrel Field Afferents From The Thalamus 47

Response Properties In The PMBSF 48

What Animals Have Mystacial Vibrissae? 53

What Is whisking? 55

What Do Animals Use Their Whiskers For? 61

How Sensitive Are The Whiskers? 67

Sensory Guidance Of Movement 69

Visually-Guided Jumping As A Model Of The Sensory Guidance Of Movement 69

Whisker-Guided Jumping As A Model Of The Sensory Guidance Of Movement 71

Possible Pathways Underlying The Sensory Guidance Of Movement 74

The Discovery Of Functional Specialisation In The Brain 74

Cortico-Cortical Connections And The Guidance Of Movement 75

Experiments Exploring The Function Of The Cortico-Cortical Fibres 76

The Split Brain 77

The Basal Ganglia 82

Motor Disorders Due To Basal Ganglia Dysfunction 83

Are The Basal Ganglia Involved In The Sensory Guidance Of Movement 84

The Cerebellum 85

The Cortico-Pontine Pathway 85 
Visual Responses In Pons 88

Somatosensory Cortical Efferents 90

Cortico-Thalamic Projections 90

Cortical Influence On The Thalamus. 91

Cortico-Pontine Projections 93

Cortico-Striate Projections 94

Cortico-Tectal Projections 95

Cortico-Trigeminal Projections 95

Cortico-Cortical Fibres 96

The Cortico-pontine Pathway 97

Organisation Of The Cortico-pontine Terminals In The Pontine Nuclei 99

Sensorimotor Input To Pons In The Rat 100

Pontine Efferents 101

Cerebellar Connections With Motor Areas Of The Brain 102

\section{MATERIALS AND METHODS 104}

Principles Of Experimental Techniques 105

The Wheat Germ Agglutinin - Horse Radish Peroxidase (WGA-HRP) 106

Biotinylated Dextran-Amines Method 108

Cytochrome Oxidase Method 109

Behavioural Studies 111

Subjects 111

Apparatus 111

The Testing Room 112

Dark Imaging Equipment 112 
Training 115

Surgical Procedures 120

Surgical Anaesthesia 120

Stereotaxic Procedures 120

Cerebral Peduncle Lesion 120

Injection Of Neuroanatomical Tracer WGA-HRP Into The Pontine Nuclei 122

Injection Of Neuroanatomical Tracers Into The PMBSF 123

Whisker Removal 124

Perfusion 125

Perfusion Of WGA-HRP Injected Animals 125

Perfusion Of BDA Injected Rat 126

Histological Procedures 127

Tissue Sectioning 127

Neuronal Tracer Histology 129

Treatment Of HRP Containing Tissues 129

TMB 129

DAB 130

Biotinylated Dextran Amines 131

Cell, Myelin And Enzyme Histology 131

Fibre Stain 131

Nissl Stain 132

Cytochrome Oxidase 133

Analysis Of Data 134

Behavioural Data 134

Anatomical Data 134 
Injection Sites 135

Lesion Sites 136

Cellular And Terminal Label 136

\section{RESULTS 138}

Case 1139

Case II 145

Case III 154

Case IV 162

Case V 171

Case VI 180

Case VII 192

Case VIII 195

\section{DISCUSSION 198}

Experimental Results 201

Other Possible Reasons For The Deficit 207

Differences Between Vibrissal And Visually Guided Jumping 210

APPENDIX 1219

Surgical Protocol 220

Individual Peduncle Lesion Co-ordinates 220

Individual Pons Injection Co-ordinates 220

Individual PMBSF Injection Co-ordinates 221

Histological Protocol 222

Horse-Radish Peroxidase Histology 222

Fixation 222 
Section Processing 223

Tetramethylbenzidine (TMB) Chromogen 223

3,3' - Diaminobenzidine (DAB) Chromogen 226

Biotinylated Dextran Amine (BDA) Histology 227

Fixation 227

Section Processing 227

Reaction 227

Fibre Stains 228

Nissl Stains 229

Cytochrome Oxidase 230

APPENDIX $\| 232$

Other Work Carried Out During the Writing Of This Thesis 233

Response Properties Of Barrel Field Neurons In The Awake Behaving Rat 233

Short-Term Enriched Experience Potentiates Whisker-Pairing plasticity of Barrel Cortex

Neurones In Adult Rats 236

REFERENCE LIST 239 
INTRODUCTION 


\section{What Are Vibrissae?}

"So large and particular distribution of an exquisitely sensible nerve, it is reasonable to suppose, must be for the purpose of some sensible function." - James Broughton (1823)

Many classes of mammals possess large sensory whiskers distributed over several locations on their body surface (Lyne, 1959; Pocock, 1914). In rats these whiskers are known as sinus hairs or vibrissae. They are complex mechanoreceptors for vibro-tactile stimuli.

The rat has numerous sensory whiskers. The largest and most obvious of these being an array of around 30 vibrissae in the mystacial pad on the upper lip of the muzzle. These whiskers form a well organised sensory organ and are known as the mystacial (moustache like) vibrissae. Other sensory vibrissae exist, either in isolation or in groups of two or three at other locations on the body. These include the supraorbital, postorbital, lateral cervical, median cervical and submental vibrissae, as well as on the carpel joint of the forelimb (Pocock, 1914; Vincent, 1913). These specialised sensory hairs can be anatomically disassociated from non-specialised, non-sensory, "fur" hairs that cover most of the body of the rat in several ways.

- Size and structure of hair shaft.

- Size and structure of the hair follicle.

- Neural innervation of the hair follicle.

- Blood supply to the hair follicle.

In addition to these qualities the mystacial vibrissae of the rat have some additional, unique features separating them from the other large sensory vibrissae. 
- The whiskers are mobile and under voluntary motor control.

- Each whisker has a one to one somatotopic representations within the central nervous system.

\section{The Mystacial Vibrissae}

Figure 1 shows the mystacial vibrissae of the rat. The mystacial vibrissae are remarkable for their size and by their clear organisational pattern. They are found in the mystacial pad on the upper lip of the rat. Horizontal rows of vibrissae run parallel to the lip on either side of the snout. Vincent (Vincent, 1913) observed six rows of whiskers with five to nine vibrissae follicles in each row. The sixth row lies along the inferior boundary of the mystacial pad, Vincent located the sixth row by following the neural innervation to the follicle of this row, but the associated whiskers can be small in number and size making them hard to identify. In the modern literature five rows are normally described, with four to seven whiskers in each row. The rows of whiskers have been classified as rows A E. Row E being most ventral and row A most dorsal. Individual whiskers are numbered according to their position, whisker 1 being the most caudal, and the whisker number increasing rostrally (Zucker and Welker, 1969). There are also four large vibrissae located immediately caudal to rows $A, B, C$, and $D$. These whiskers do not belong to any of the rows, the whiskers being slightly offset in a ventral direction so that they sit between the rows (Danforth, 1925). Because of their position the whiskers are called straddlers and have been classified as whiskers $\alpha, \beta, \gamma$, and $\delta$. The $\alpha$ straddler whisker is the most dorsal, the $\delta$ straddler the most caudal (Van der Loos and Woolsey, 1973). 
The whisker follicles of the rat are seen on a gross level very early in development. The adult layout of the follicles on the mystacial pad is obvious as early as El6 (Beaudoin, 1980). The anatomy and morphology of vibrissae and mystacial pad of both rats and mice have been intensively studied, though much of the work on the development of the whiskers has been performed solely on mice. There are no major differences in the organisation of the whiskers, or mystacial pad between these species.

\section{Structure Of The Vibrissae And Follicle}

The base of the shaft of each mystacial vibrissae is buried in a complexly innervated, spindle-shaped structure. The structure is made up of an epidermally derived follicle core with a surrounding dermally derived, encapsulated blood vascular sinus (Vincent, 1913). The blood sinus gives vibrissae their alternative name of sinus hair (Andres, 1966). Due to the complicated anatomy of this structure that differentiates it from common hair follicles it has been called a follicle-sinus complex (F-SC) (Rice et al., 1986)

A follicle-sinus complex is shown in Figure 2. The epidermally-derived portion of the F-SC is comprised of two sheaths. The internal-root sheath (IRS) that surrounds the vibrissae shaft and an external-root sheath (ERS) that envelops the IRS. The ERS is separated from the dermally derived blood sinus of the F-SC by a basement membrane called the glassy membrane (Rice et al., 1986; Vincent, 1913). The crater created by the entry of the vibrissae into the follicle at the surface of the skin is referred to as the rete ridge collar and contains Merkel cells (Halata and Munger, 1980; Munger and Halata, 1983). Merkel cells are specialised sensory receptors. They are found in conjunction with 
Merkel endings or discs, which are expanded nerve terminals. Merkel cells are slowly adapting mechanoreceptors and have small receptive fields.

A sheath of loose connective tissue surrounds the dermally-derived portion of the F-SC. The sheath is called the mesenchymal sheath. The entire F-SC structure is separated from the surrounding loose connective tissue and contained by a compact capsule of collagenous tissue. The mesenchymal sheath and capsule fuse together at the bottom of the F-SC below the vibrissae shaft to seal up the deep end of the F-SC. At the deep end of the F-SC is the bulb of the developing vibrissae that contains some dermal components, the dermal papilla. The mesenchymal sheath and the capsule rejoin at the neck of the hair shaft to seal the superficial end of the sinus. The cone like structure created by this joining is referred to as the conus. The mesenchymal portion being the inner conical body and the capsular the outer conical body (Andres, 1966; Renehan and Munger, 1986; Vincent, 1913).

The sinus receives its blood supply from an artery that enters the F-SC at about the lower third of the capsule. The sinus is split into two parts. The deeper portion of the F-SC contains a matrix of trabeculae, this is called the cavernous sinus. The superficial part is an open space and is referred to as the ring sinus. At this point the ERS and glassy membrane surrounding the vibrissae shaft are thick and have a thick collar of connective tissue called the ringwulst circling them suspended on the mesenchymal sheath. Blood drains from the F-SC via a plexus of small veins near the conus. The size of the mystacial vibrissae and their associated F-SC increase rostro-caudally on the whisker pad (Vincent, 1913). 


\section{Neural Innervation}

Sensory endings are located at six positions in the F-SC: i) the epidermal rete ridge collar, ii) the inner-conical body, iii) the mesenchymal sheath at the level of the ring sinus, iv) the ERS at the level of the ring sinus, v) the mesenchymal sheath and the trabeculae at the level of the cavernous sinus, and vi) the dermal papilla of the bulb. The last of these sites has been shown to receive only a small innervation in the rat. The innervation of the cavernous and ring sinus comes from the large-deep (or follicular) nerve. The innervation of the rete ridge and inner-conical body comes from several small-superficial vibrissal (or conus) nerves (Renehan and Munger, 1986; Rice et al., 1986).

\section{Deep-Vibrissal Nerves}

A single deep-vibrissal nerve enters the postero-dorsal aspect of each F-SC with the associated arteriole at the level of the cavernous sinus. These nerves are a branch of a major fascicle of the infraorbital nerve. These fascicles are called row nerves and they lie in the dermis between the rows of F-SCs below the level of the vibrissal papillae. Each row of F-SCs receive their deep vibrissal nerve from the row nerve immediately dorsal to it (Dorfl, 1985; Vincent, 1913).

The deep-vibrissal nerve ascends the follicle breaking into smaller bundles as it traverses the cavernous sinus. These bundles disperse around the follicle (Vincent, 1913). Some of these bundles innervate the cavernous sinus but most continue to the level of the ring 
sinus. At the ring sinus the bundles separate into axons and distribute around the mesenchymal sheath as sensory endings (Renehan and Munger, 1986; Rice et al., 1986).

The Superficial-Vibrissal Nerve

The row nerves also provide many small nerves to the skin. Originally known as the conus nerve these ascend close to the F-SCs dorsally and ventrally (Andres, 1966; Tello, 1923). These skin nerves divide just below the epidermis and supply the superficial vibrissal nerves to the F-SCs as well as the plexus of nerves that supply the innervation to the inter-vibrissal fur (Dorfl, 1985; Rice et al., 1986). Up to six superficial vibrissal nerves innervate each F-SC, each nerve contains 5-15 axons. Superficial vibrissal nerves can innervate more than one F-SC in the same row but they never innervate F-SCs in other rows. The superficial-vibrissal nerve supplies ascending branches to the rete ridge collar and descending branches to the inner-conical body (Rice et al., 1986).

\section{Receptor Types And Distribution}

Ascending branches of the superficial vibrissal nerve innervate the rete ridge collars of sinus hairs. Merkel endings from this innervation completely encompass the mouth of the vibrissal follicle (Halata and Munger, 1980; Munger and Halata, 1983; Rice et al., 1986). 
The INNER-CONICAL BODY is almost entirely innervated by descending fibres from the superficial-vibrissal nerve. Sporadically fibres are seen to cross from the ring sinus to the inner-conical body but on the whole the innervation of these two structures are separate (Rice et al., 1986). Some studies do not find any cross over between the innervation between these two structures (Renehan and Munger, 1986). the innerconical body of the rat contains a dense plexus of axons and neuronal terminals. These fibres circle the follicle forming a hollow column containing the hair shaft throughout the extent of the inner-conical body. The fibres terminate in lanceolate and blebbed endings. Lanceolate endings are restricted to hair follicles. They are fine, flattened endings of myelinated fibres. They are found in the follicles of down, guard and vibrissal hair. The blebbed endings belong to unmyelinated fibres. The fibres terminate in a series of abrupt cytoplasmic swellings (Mosconi et al., 1993).

The deep-vibrissal nerve supplies innervation to the lower part of the F-SC, below the level of the inner-conical body. This nerve supplies the densest innervation in the F-SC, found in the ring sinus. The mesenchymal sheath between ringwulst and the conus is innervated by lanceolate endings. These individual flattened-blade-like endings are arranged in parallel to the vibrissae shaft. As a group they circle the shaft in the manner of the pickets of a fence (Rice et al., 1986).

Merkel endings innervate the external-root sheath throughout the level of the ring sinus. These cross the glassy membrane and terminate amongst the Merkel cells that are found on the external-root sheath between the ringwulst and the conus. The axons that supply the endings run round the follicle's circumference, and are therefore orientated perpendicularly to the lanceolate endings (Rice et al., 1986). 
The cavernous sinus is sparsely innervated. Some fibres from the deep-vibrissal nerve contribute to a sparse plexus of axons and terminals that are distributed in the mesenchymal layer and throughout the connective tissue trabeculae that traverse the sinus. The majority of axons terminate as free-nerve endings, these axons are finecalibre fibres, some Ruffini endings have been reported. In the rat corpuscular endings are seen in the cavernous sinus (Rice et al., 1986).

Information gathered by the whiskers in the periphery is carried to the central nervous system within the trigeminal nerve, the fifth cranial nerve (Vincent, 1913). 

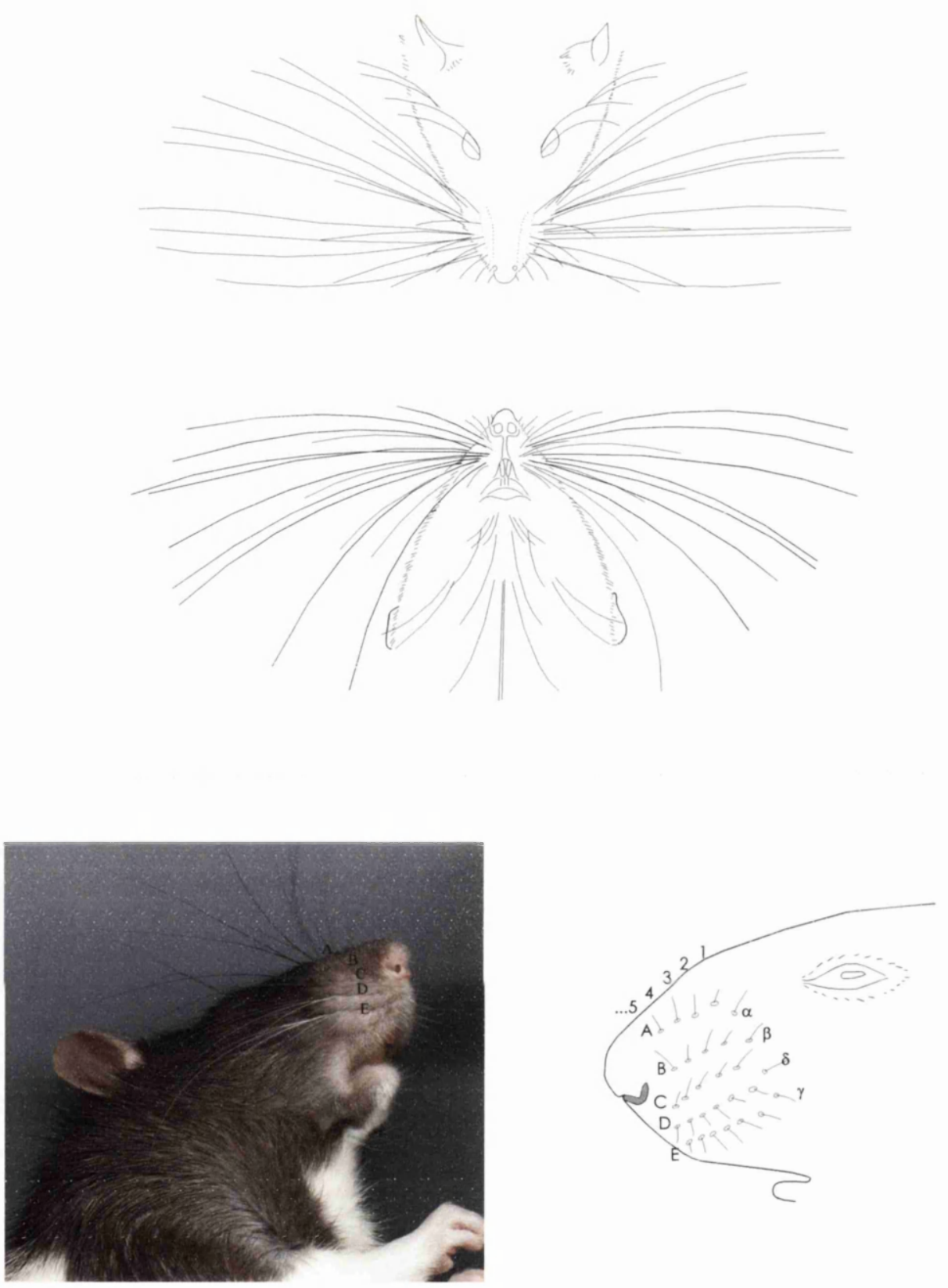

Figure 1:

Organisation of the vibrissae on the face of the rat, as seen from above (top) and below (middle).

Schematic of the organisation and nomenclature of the vibrissae on the mystacial pad (bottom). 


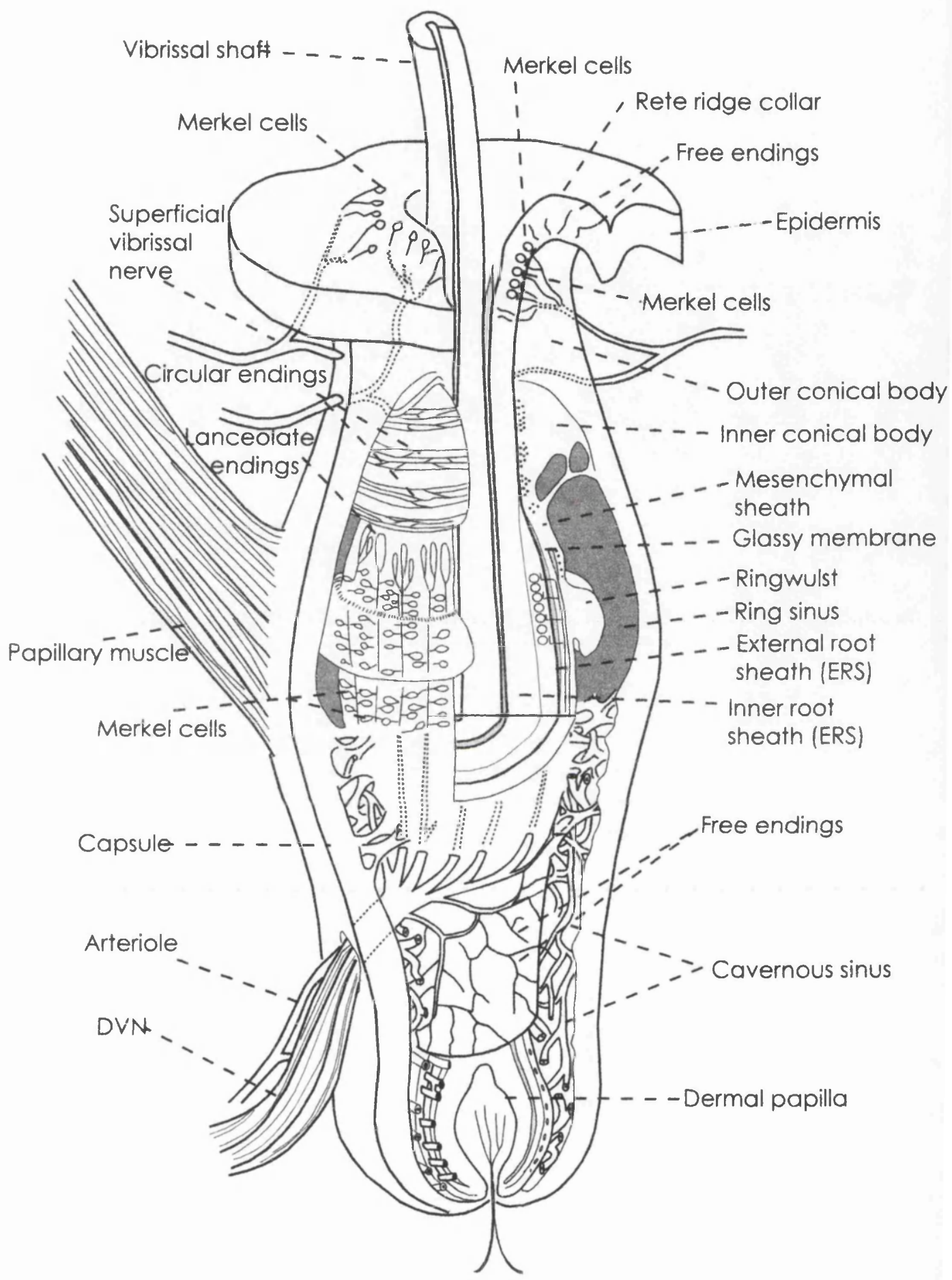

Figure 2:

A schematic, three dimensional view of a vibrissal follicle-sinus complex (FS-C) like that of a adult rat. Adapted from Rice et al., 1993. 


\section{The Trigeminal System}

\section{The Trigeminal Nerve And Ganglion}

The primary sensory afferents from a large portion of the face and head run in the sensory trigeminal nerve. The sensory trigeminal nerve is split into three divisions; ophthalmic, maxillary and mandibular. The dorsum of the head including the supraorbital vibrissa, the cornea and conjunctiva, the glabrous and hairy skin over the dorsum and tip of the nose and the intranasal mucosa are innervated by the ophthalmic division. The maxillary division supplies the upper lip, the postorbital skin, the lateral nose, the intraoral-upper-jaw mucosa and the upper teeth. The mandibular division supplies the temporomandibular joint, the skin over the mandible, the lower lip, the intraoral-lower-jaw mucosa, and the teeth and anterior tongue. The caudal head and pinna of the ear are supplied by $\mathrm{C} 2$ and $\mathrm{C} 3$ from the dorsal rami and cervical plexus [Waite, and Tracey, 1995).

The infraorbital nerve of the maxillary division of the trigeminal nerve supplies sensory innervation to the mystacial vibrissae. Vincent (Vincent, 1913) pointed out that the nerve is very large, containing an estimated $15000-20000$ fibres as it leaves the infraorbital foramen. Vincent's count is probably an underestimate of the total number of fibres as it would be unlikely that she could have resolved the unmyelinated fibres whose diameters are at the limits of the resolution of light microscopy. The infraorbital nerve is, in fact, the largest peripheral nerve found in the mouse (Dorfl, 1985). Vincent (Vincent, 1913) remarked that animals with sensitive vibrissae and specialised facial sensory structures i.e. the tapir and the elephant share this marked enlargement of the 
infraorbital nerve of maxillary division of the fifth nerve. All of the fibres that innervate the F-SCs of the mystacial pad are found in this nerve, their cell bodies are found in the trigeminal (semilunar or Gasserian] ganglion. The trigeminal ganglion is found in the middle-cranial fossa of at the base of the skull (Vincent, 1913). The trigeminal ganglion contains all the cell bodies of the fibres that make up the trigeminal nerve, a total of about 50000 neurones, the maxillary and the ophthalmic divisions of the ganglion are indistinguishable, the mandibular division lies laterally (Forbes and Welt, 1981). Like dorsal root ganglia the neurones in the trigeminal ganglion are pseudounipolar and have their central terminations in the sensory trigeminal nuclei in the brain stem.

\section{Response Properties In The Trigeminal Ganglion}

Neurones in the trigeminal ganglion that innervate the mystacial vibrissae encode for the amplitude, velocity, duration, frequency and angular direction of vibrissal movement (Fitzgerald, 1940; Gibson and Welker, 1983a; Gibson and Welker, 1983b; Zucker and Welker, 1969). The remarkable sensitivity of the vibrissal system demonstrated by behavioural testing of rats (Carvell and Simons, 1990; Guic-Robles et al., 1989; Hutson and Masterton, 1986) is reflected in the sensitivity of some of the response properties found in first order sensory trigeminal neurones. Neurones have been found that respond to whisker deflections as small as $0.1^{\circ}$ (Gibson and Welker, 1983a), in the cat some first order sensory trigeminal neurones will discharge in phase with a vibrating stimuli at frequencies of up to $1500 \mathrm{~Hz}$ (Gottschaldt and Vahle-Hinz, 1981).

Cells of the trigeminal ganglion that respond to movement of the mystacial vibrissae do so to only one whisker. The cells do not display any spontaneous discharge (Zucker and 
Welker, 1969). Vibrissal-responsive cells of the trigeminal ganglion fall roughly into three categories. Many are slowly adapting neurones, although the estimated size of this population differs among studies. Gibson and Welker (1983a) have it at 45\%; Zucker and Welker (1969) at 60\%; and Lichtenstein et al. (1990) at 75\%. These slowly adapting neurones are likely to have a strong directional preference. Most of the remaining vibrissal sensitive first order trigeminal neurones have fast-adapting responses to whisker displacement. These fast-adapting cells show little directional selectivity. The smallest group (<10\%) require large deflections or deflections of high velocity to elicit a response (Gibson and Welker, 1983a; Gibson and Welker, 1983b; Lichtenstein et al., 1990; Zucker and Welker, 1969).

These populations of neurones with different response properties have been related to the receptor types in the vibrissa follicles. Merkel endings are thought to contribute to the slowly adapting directionally sensitive cells. Lanceolate endings are responsible for the rapidly adapting responses non-directional responses (Rice et al., 1986). It is interesting to note that vibrissal response properties of cells in the trigeminal ganglion are similar across a range of animal species (Fitzgerald, 1940; Pubols, Jr. et al., 1973; Zucker and Welker, 1969). Dykes (1975) even found that animals living in disparate environments such as the cat and the seal display similar trigeminal ganglion vibrissal response properties.

\section{Somatotopy In The Trigeminal Ganglion}

The cells in the trigeminal ganglion are approximately somatotopically organised. Neurones innervating ophthalmic skin lie anteromedially and those innervating 
mandibular skin are situated posterolaterally. Dorsal peripheral regions whose sensory axons are carried in the ophthalmic division of the trigeminal nerve are represented dorsally in the ganglion, ventral periphery innervated by the mandibular branch ventrally. The cell bodies of the maxillary branch are located in between (Arvidsson, 1977; Darian-Smith, 1973; Gregg and Dixon, 1973; Martin and Dolivo, 1983). Using electrophysiological mapping Zucker and Welker (1969) found that within this whole body somatotopy there is a somatotopy of cells whose fibres innervate individual vibrissae. Cells representing most ventral vibrissal row $E$ are found laterally in the ganglion, the most dorsal row $A$ is represented ventrally with rows $B, C$ and $D$ in the middle. The vibrissal somatotopy was later demonstrated anatomically by Arvidsson (1982). After applying HRP directly to the vibrissal nerves in the periphery Arvidsson (1982) found groups of labelled cells in the trigeminal ganglion corresponding to the somatotopy reported by Zucker and Welker (1969). All reports suggest that the somatotopy in the trigeminal ganglion is not precise and there is a considerable overlap between the representations.

\section{The Trigeminal-Nuclear Complex}

The central terminals of the trigeminal ganglion lie in the trigeminal-nuclear complex located in the brain stem. These are subdivided into three main nuclei: the mesencephalic nucleus (Me5), the main or principle-sensory nucleus (Pr5), and the spinal nucleus (Sp5). The trigeminal complex also includes the motor trigeminal nucleus (Mo5), the supratrigeminal nucleus (Su5), the intertrigeminal nucleus, and the paratrigeminal nucleus (Darian-Smith, 1973). 
The principal and spinal trigeminal nucleus receives vibrissal afferents from the trigeminal ganglion via the trigeminal sensory root. The spinal nucleus is itself subdivided into three subnuclei: subnucleus caudalis ( $\mathrm{Sp} 5 \mathrm{C}$ ], subnucleus interpolaris (Sp5I), and the subnucleus oralis (Sp5O). Intratrigeminal fibres interconnect all the subnuclei. These form fibre bundles that are characteristic of the spinal nuclei (Darian-Smith, 1973).

A diagram of the trigeminal-nuclear complex with a summary of the major outputs is given in Figure 3.

The Principle-Sensory Nucleus

The principle-sensory nucleus is the most rostral part of the trigeminal-nuclear complex. It lies in the lateral pons. In transverse section it is roughly kidney shaped. It begins at the same rostral pole as the more medial motor-trigeminal nucleus and extends caudally to the level of the facial nucleus where it is continuous with the rostral end of the subnucleus oralis of the spinal trigeminal nucleus (Bates and Killackey, 1985; DarianSmith, 1973; Fukushima and Kerr, 1979). The principle-sensory nucleus contains many small and medium-sized neurones, as well as a few large neurones, especially in the more dorsal portion of the nucleus (Bennett-Clarke ef al., 1992).

The main efferent target from the principle-sensory nucleus is the contralateral thalamus (Darian-Smith, 1973). The principle-sensory nucleus contributes by far the largest input to the thalamus from any division of the trigeminal system (Bruce et al., 1987; Chiaia et al., 1991a; Mantle-St.John and Tracey, 1987). The thalamic projection originates mainly from the small and medium-sized cells and is directed to ventroposteromedial (VPm) 
and posterior (PO) nuclei of the thalamus (Bruce et al., 1987; Chiaia et al., 1991b; Fukushima and Kerr, 1979; Peschanski, 1984). An ipsilateral projection from the principlesensory nucleus to the thalamus has been described in rats by some authors (Fukushima and Kerr, 1979; Patrick and Robinson, 1987) but when it is seen, is very small compared to that in other species i.e. cat and human, and is not reported at all by many investigators. Other efferents arising in principle-sensory nucleus terminate in the cerebellum (Huerta et al., 1983; Mantle-St.John and Tracey, 1987; Patrick and Robinson, 1987: Watson and Switzer, 1978), the superior colliculus (Bruce et al., 1987; Huerta et al., 1983), the inferior olivary nucleus (Huerta et al., 1983), the gracile nucleus, the zona incerta (Shammah-Lagnado ef al., 1985) and the hypoglossal nucleus (Aldes and Boone, 1985).

Trigeminothalamic neurones in the principle-sensory nucleus mainly respond to stimulation of a single vibrissae (Nord, 1968; Shipley, 1974). Jacquin and his colleagues (1988) found that the majority of cells in principle-sensory nucleus were responsive to vibrissae stimulation (69\%). Of these most (80\%) were sensitive to only one vibrissae, and a large number of these had a directional preference (40\%). The response properties found in the principle-sensory nucleus reflect those found in their primary afferents from the trigeminal ganglion.

\section{The Spinal Trigeminal Subnucleus Oralis}

Subnucleus oralis begins immediately caudal to the principle-sensory nucleus and is coextensive rostro-caudally with the facial nucleus (Darian-Smith, 1973; Fukushima and Kerr, 1979). Very large cells (30 - 50 microns) dominate the oralis subnucleus, with some 
medium and small cells. Cells in the subnucleus oralis tend to have large dendritic trees (Fukushima and Kerr, 1979; Jacquin and Rhoades, 1990).

Neurones in the oralis nucleus respond to vibrissae stimulation. The receptive field sizes of the cells ranges from single vibrissae to the whole mystacial pad. About half have punctate fields such as a single vibrissae /Gibson, 1987; Jacquin and Rhoades, 1990; Nord, 1968; Shipley, 1974).

The subnucleus oralis provides a large input to the facial nuclei (Erzurumlu and Killackey, 1979). Neurones also project to VPm of the thalamus, the cerebellum, the other trigeminal nuclei and the spinal cord (Jacquin and Rhoades, 1990), the superior colliculus (Bruce et al., 1987) as well as zona incerta (Shammah-Lagnado et al., 1985), and the anterior-pretectal nuclei (Yoshida et al., 1992).

\section{The Spinal Trigeminal Subnucleus Interpolaris}

Although the principle-trigeminal nucleus provides the main input to the thalamus, a projection to thalamus arises form the subnucleus interpolaris of the spinal-trigeminal nucleus. The subnucleus interpolaris continues from the caudal end of subnucleus oralis. It starts rostrally at the level of the caudal facial nucleus and continues to the level of the obex, where the substantia gelatinosa begins. At its caudal end it forms an oblique boundary with Subnucleus caudalis (Darian-Smith, 1973; Fukushima and Kerr, 1979; Phelan and Falls, 1989). The Subnucleus interpolaris mostly contains small and mediumsized neurones with a few large cells (Bates and Killackey, 1985) 
The Subnucleus interpolaris projects to the thalamus (Bruce et al., 1987; Chiaia et al., 1991a; Erzurumlu et al., 1980; Fukushima and Kerr, 1979; Mantle-St.John and Tracey, 1987; Peschanski, 1984). Like the principle nucleus the subnucleus interpolaris has a direct projection to the cerebellum and an indirect projection via the inferior olivary nucleus. The subnucleus interpolaris also projects to the superior colliculus (Bruce et al., 1987; Huerta et al., 1983; Mantle-St.John and Tracey, 1987; Watson and Switzer, 1978). The anterior-pretectal nuclei (Yoshida et al., 1992). The zona incerta (ShammahLagnado et al., 1985) also receives a projection from the interpolaris subnucleus. Some subnucleus interpolaris cells have bifurcating projections; to the superior colliculus and the thalamus (Bruce et al., 1987; Jacquin et al., 1986), the olivary nuclei and superior colliculus (Huerta et al., 1983), and the thalamus and the cerebellum (Mantle-St.John and Tracey, 1987; Patrick and Robinson, 1987).

Cells of the Subnucleus interpolaris subnucleus can be split into two categories, projection neurones and local-circuit neurones. Local-circuit neurones of the subnucleus interpolaris respond to several modalities, vibrissae, nociceptors, guard hairs and periodontia. About half of the local-circuit neurones respond to only vibrissal stimulation and virtually every one of these responds only to one vibrissae (Jacquin et al., 1989). Projection neurones are functionally and structurally distinct from local-circuit neurones. The majority of projection neurones respond to vibrissal stimulation and most of these have multi-vibrissal receptive fields. Thalamic projecting neurones have larger receptive fields than cerebellar projecting neurones. A small number of cerebellar projecting neurones display directional selectivity, thalamic-projecting cells never exhibit this quality. Collicular and olivary projecting neurones have multi-vibrissal receptive fields without velocity or directional sensitivity (Jacquin et al., 1989; Jacquin ef al., 1986; Woolston et al., 1982). 


\section{The Spinal Trigeminal Subnucleus Caudalis}

The Subnucleus caudalis starts at the level of the obex and continues caudally to the level of the upper cervical dorsal horn, with which it is continuous. Like the dorsal horn the Subnucleus caudalis is laminated. The layers are numbered $1-4$ dorso-ventrally, marginal, substantia gelatinosa, and the two magnocellular layers (layers 3 and 4) respectively (Darian-Smith, 1973; Fukushima and Kerr, 1979).

Layer 1 projects to many areas of the contralateral thalamus including VPm, the gelatinous (submedius] nucleus, the intralaminar nucleus and the posterior nuclei (Fukushima and Kerr, 1979; Yoshida ef al., 1991). Layers 1 and 2 project to the solitary tract and the zona incerta (Menétrey et al., 1992; Shammah-Lagnado et al., 1985). The magnocellular layer send fibres to the facial nuclei (Erzurumlu and Killackey, 1979) and to the other more rostrally placed trigeminal nuclei (Jacquin et al., 1990).

\section{Somatotopy In The Trigeminal-Nuclear Complex}

There is an overall somatotopy of the terminations of the first order neurones in the trigeminal-nuclear complex. In the principle nucleus and the interpolaris and oralis subdivisions of the spinal nuclei the mandibular afferents end dorsally and the ophthalmic ventrally. In the subnucleus caudalis mandibular endings are found more dorsomedially than the ophthalmic which are more ventrolateral. In all cases the 
maxillary endings are located between the other two representations (Darian-Smith and Mayday, 1960; Grant and Arvidsson, 1975; Marfurt, 1981; Nord, 1967). There is also a medial-lateral somatotopy. In the principle sensory nucleus, the subnucleus oralis and subnucleus interpolaris representation of caudal skin are found laterally and representations of the nose and rostral face are found medially (Nord, 1967; Waite and Cragg, 1982). The pattern is different in Subnucleus caudalis where the nose and rostral face terminate rostrally and the more caudal regions terminate in more caudal parts of the nucleus (Arvidsson, 1982).

The most obvious somatotopic representation in the trigeminal-nuclear complex is found for the central terminations of vibrissal afferents from the trigeminal ganglion for individual F-SCs and is located in the spinal subnuclei and principle nuclei of the trigeminal-nuclear complex (Arvidsson, 1982; Nord, 1967) as shown in Figure 4. In transverse section the vibrissa are represented in a pattern of primary-trigeminal afferents that mimic the arrangement of the vibrissae in the periphery. The whisker representations are especially easy to visualise in young animals, and are best localised with metabolic stains such as succinic dehydrogenase or cytochrome oxidase. Each vibrissa is represented by a group of cells called a barrelette. These representations are found in the principle sensory nucleus, the subnucleus interpolaris and the subnucleus caudalis. Subnucleus oralis receives vibrissal input but barrelettes are not found (Belford and Killackey, 1979; Ma, 1991; Ma and Woolsey, 1984). The relationship between a single vibrissae and its representation in the trigeminal-nuclear complex of an adult rat was strikingly demonstrated by Arvidsson (1982). After applying horse-radish peroxidase conjugated with wheat-germ agglutinin (WGA-HRP) directly to the vibrissal nerves Arvidsson found that the tracer was transported transganglionically to reveal the discrete patch of primary trigeminal terminals representing individual vibrissae. 
The representation for a single vibrissae is continuous in the rostro-caudal axis. So the primary-trigeminal afferents of a single whisker terminate in a column running throughout the nuclei (Bates and Killackey, 1985; Ma, 1991). Jacquin and his colleagues (Jacquin et al., 1993) demonstrated this columnar vibrissal representation by allowing a hamster to behave freely whilst exposed to the metabolic label 2 deoxy-glucose after removing all but one whisker on either side of the snout, thus revealing the representation of a single whisker running throughout the trigeminal-nuclear complex. Staining did not occur in the oralis subnucleus.

\section{Targets Of The Trigeminal-Nuclear Complex}

As described above, the trigeminal-nuclear complex projects to many targets (see Figure 3). The major targets are the cerebellum, the superior colliculus, and the cortex (via the thalamus). Each of these structures is considered to have very different functional rôles. Are there any differences in the populations of neurones that project to them?

\section{Trigeminal-Nuclear Complex Efferents To The Superior Colliculus}

The superior colliculus receives the majority of its vibrissal projections from the contralateral subnucleus interpolaris. The superior colliculus also receives a smaller projection from the subnucleus oralis and an even smaller projection from the principle- 
sensory nucleus and subnucleus caudalis. These projections mainly terminate in the intermediate and deep layers of the superior colliculus (Bruce et al., 1987; Huerta et al., 1983; Killackey and Erzurumlu, 1981). The projection from the principle nucleus is bilateral whilst the projection from the interpolaris subnucleus is unilateral. The vibrissal representation in the deep layers of the colliculus has been shown to be in register with the part of the retinotopic visual field representation in the superficial layers which the whiskers cross, when they pass in front of the eyes (Dräger, 1975a; Dräger and Hubel, 1975b). These superimposed intermodal fields are thought to be important for orientation responses, a behaviour in which the superior colliculus plays an important rôle. After injections of a retrograde tracer into the superior colliculus there is a bias of the distribution of labelled cells to the ventro-lateral aspect of the trigeminal-nuclear complex (Bruce ef al., 1987). These areas have been shown to contain the vibrissal representation (Belford and Killackey, 1979; Ma, 1991).

Bruce et al. (Bruce et al., 1987) showed that trigeminal cells that project to the superior colliculus constitute a morphologically distinct sub population of cells regardless of the subdivision of the trigeminal-nuclear complex in which they are found. They are typically amongst the largest cells, and have multipolar dendritic fields. These features are most prominent in the subnucleus oralis but are observable in all sub-divisions. After Bruce et al. (Bruce et al., 1987) made paired injections of fluorescent tracers into thalamus and superior colliculus a sprinkling of double labelled cells are found throughout the trigeminal-nuclear complex. These cells have the same morphological features as the trigeminotectal cells. Therefore, it appears that there is a small population of cells that project to the superior colliculus that also send collaterals to the thalamus. These cells are most prevalent in the ventrolateral subnucleus interpolaris. 
The superior colliculus receives a considerably smaller projection from the trigeminalnuclear complex than the thalamus. Even in the interpolaris subnucleus that provides the largest trigeminal nuclear input to the superior colliculus the number of thalamicprojection cells is about five times as large as the population of cells that project to the superior colliculus (Bruce et al., 1987).

Cells in the superior colliculus that are activated by the vibrissae usually respond to several whiskers and are rapidly adapting (McHaffie and Stein, 1981; Tiao and Blakemore, 1976). They are poorly qualified to encode for displacement, velocity or direction (McHaffie and Stein, 1981). These qualities reflect those of cells in subnucleus interpolaris, the main afferent population of vibrissal-trigeminal cells that project to the superior colliculus (Jacquin et al., 1986; Shipley, 1974).

\section{Trigeminal-Nuclear complex Efferents To The Cerebellum}

The cerebellum receives a moderate projection from the trigeminal-nuclear complex (Gould, 1980). The majority of this projection arises in the principle nucleus with a smaller but substantial projection from the interpolaris subnucleus (Steindler, 1977; Watson and Switzer, 1978). After injections of Fast Blue into the cerebellum and free horse-radish peroxidase (HRP) into the contralateral ventrobasal thalamus of ten rats, Mantle-St.John and Tracey (Mantle-St.John and Tracey, 1987) found a single double-labelled cell in the interpolaris subnucleus in the corresponding trigeminal-nuclear complex.

Although it is unwise to compare the number of retrogradely labelled cells from two structures after they have been injected with different tracers, data suggests that the 
thalamic-projection arising in the principle nucleus massively overshadows the projection from the same nucleus to the cerebellum, and the projections from any of the other sensory-trigeminal nuclei to either the thalamus or the cerebellum (MantleSt.John and Tracey, 1987).

\section{Trigeminal-Nuclear complex Efferents To The Thalamus}

By far the largest target of the trigeminal-nuclear complex is the contralateral thalamus. Trigeminal input to the thalamus arise from all parts of the trigeminal-nuclear complex. The main trigeminal input to the thalamus is from the principle nucleus, with a significant contribution from the subnucleus interpolaris. There is a very small trigeminothalamic projection from subnuclei caudalis, and oralis (Bruce et al., 1987; Mantle-St.John and Tracey, 1987). The latter was not seen in many early HRP tracing studies (Erzurumlu ef al., 1980; Fukushima and Kerr, 1979).

It would therefore appear that largely separate populations of neurones within the trigeminal-nuclear complex relay the sensory information gathered by the whiskers and encoded in the trigeminal ganglion, to the thalamus, cerebellum and the superior colliculus. These populations each respond differently to vibrissal stimulation. 

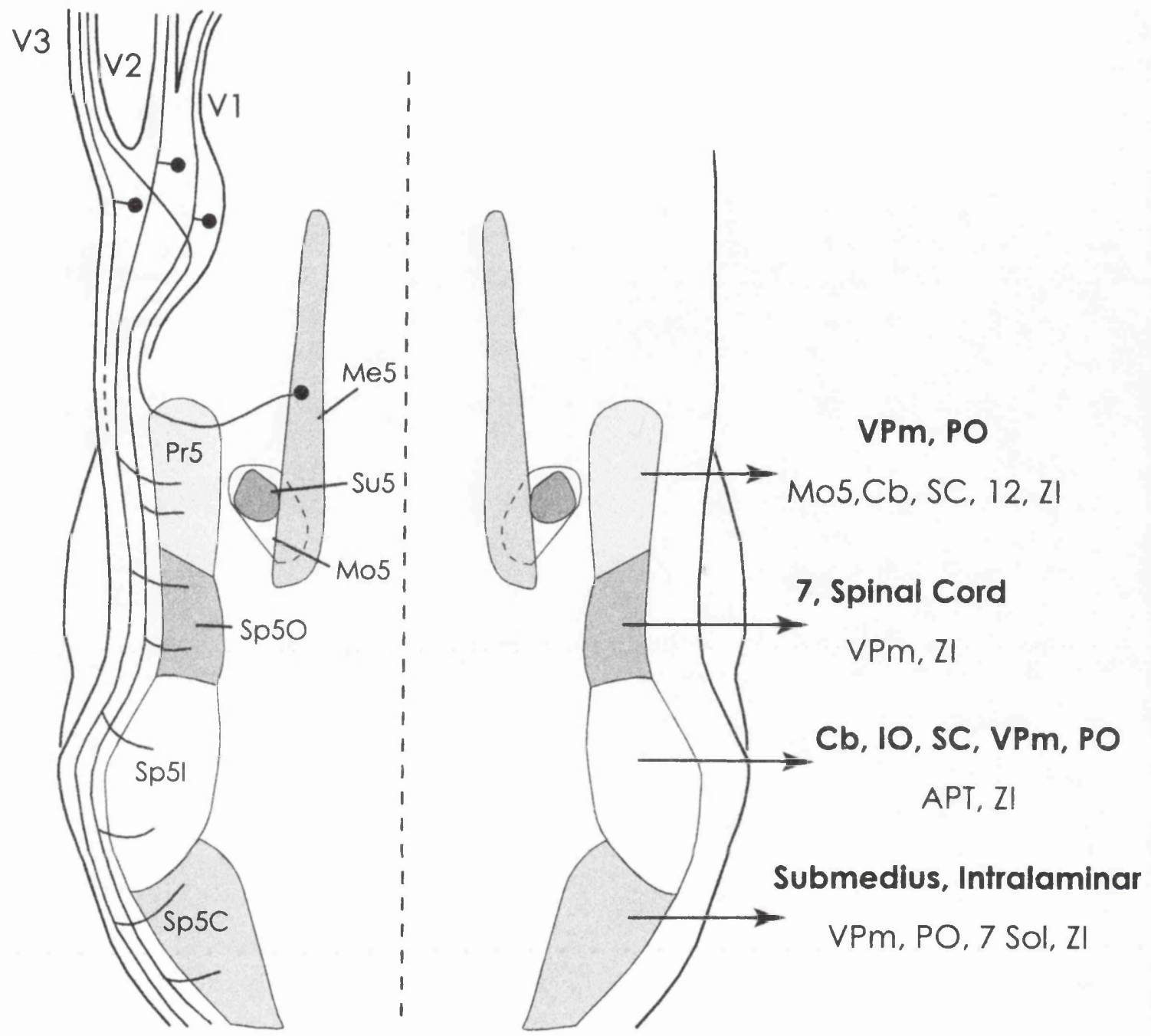

Figure 3:

Diagrammatic representation of the trigeminal brain stem nclear complex as seen from above. Inputs from the opthalmic (V1), maxillary (V2), and mandibular (V3) divisions are shown entering the trigeminal ganglion. From the ganglion they give collaterals to the to the principle nucleus $(\operatorname{Pr} 5)$, and the spinal subnuclei oralis (Sp5O), interpolaris (Sp5I) and cadalis (Sp5C). The main outputs of each nuclei are shown on the right. VPM, ventroposteromedial nucleus; PO, posterior nucleus; Cb, cerebellum; SC, superior colliculus; 7, 10, and 12, facial, vagal, and hypoglossal nuclei; APT, anterior pretectal nucleus; Sol, nucleus of the solitary tract; ZI, zona incerta. Adapted from Waite and Tracey, 1995. 


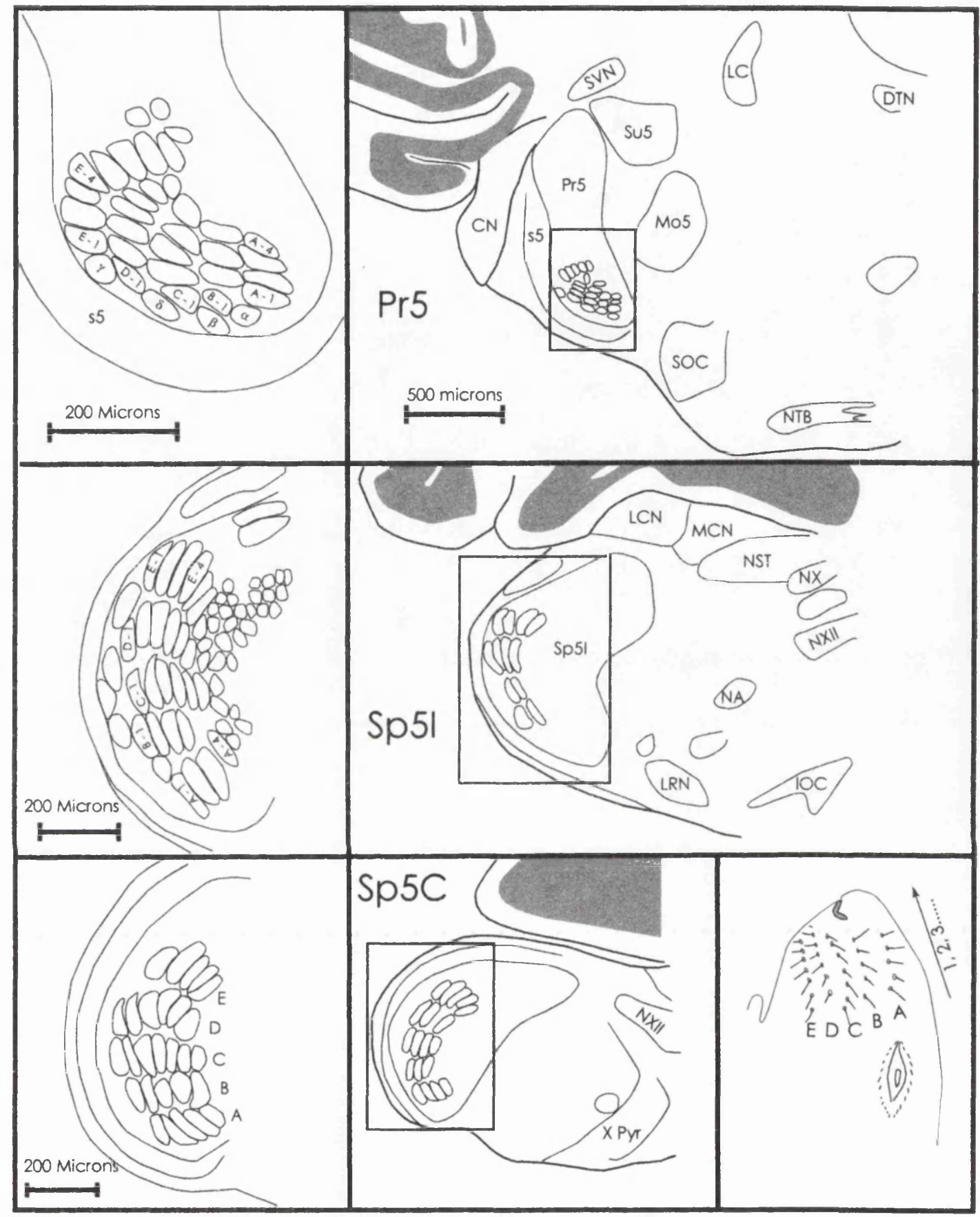

Figure 4:

Schematic drawing of representative coronal sections through the brainstem. Figures on the left show the location of the principle trigeminal nucleus (Pr5), the interpolaris (Sp5I) and caudalis ( $\mathrm{Sp5C}$ ) subnuclei of the spinal trigeminal nucleus, and the position of the barrelettes therein. The areas included in the box are shown enlarged on the left, these show the orientation of the barrelettes within each nuclei. Individual barrelettes correspond to individual whiskers in the face the position of which and nomenclature of are given in the diagram in the bottom left box. Adapted from Ma, 1991. 


\section{The Thalamo-Cortical Projection}

The main vibrissal pathway to the somatosensory cortex is through the thalamus, indeed all the subdivisions of the sensory trigeminal-nuclear complex project to the thalamus. Two thalamic areas relay vibrissal information to the somatosensory cortex the ventroposteromedial nucleus and a portion of the posterior nucleus.

\section{Ventroposteromedial Nucleus (Vpm)}

VPm lies dorsomedially to the lateral part of the ventroposterior complex of the thalamus (VPL), in transverse section it is peanut shaped. It contains thalamo-cortical relay cells, which are all medium-sized multipolar neurones. Unlike carnivores and primates, VPm of the rat contains no GABA-ergic cells or interneurones (Williams and Faull, 1987).

The sensory-afferent fibres from the trigeminal-nuclear complex are somatotopically organised within VPm. Caudal face being represented laterally and nose responses medially. As in the sensory trigeminal-nuclear complex there is an over representation of the vibrissae in this somatotopic arrangement (Land et al., 1995; Sugitani et al.. 1990; Van der Loos, 1976; Waite, 1973). Terminals of a single whisker form a discrete patch, which can be visualised with cytochrome oxidase, these have been termed "barreloids" (Van der Loos, 1976). The orientation of these barreloids within VPm is shown in Figure 5. VPm is the main target for the principle-sensory nucleus but it also has an input from the interpolaris subnucleus (Chiaia ef al., 1991a; Erzurumlu ef al., 1980; Peschanski, 1984). 
Chiaia et al. (1991a) used dimadino yellow and true blue to quantify the number of retrogradely labelled cells in the principle nucleus and interpolaris subnucleus following injections into VPm and PO. They deduced that the vast majority of cells (<90\%) in the principle nucleus project to VPm only. The majority of cells $(75 \%)$ in the interpolaris subnucleus were also found to project to VPm only. A small population of cells in the principle nucleus were double labelled $(1.4 \%)$ and therefore projected to VPm and PO. The interpolaris subnucleus providing a larger population of cells with bifurcating innervation of PO and VPm (7.4\%).

The distribution of terminals from the principle nucleus and the interpolaris subnucleus form a complementary pattern in VPm. The barreloids receive input from the principlesensory nucleus whereas the areas immediately surrounding the barreloids mainly receive a sparse input from subnucleus interpolaris (Erzurumlu et al., 1980; Smith, 1973).

Early studies of the receptive fields of vibrissae-sensitive cells in VPm found that vibrissaeresponsive cells in VPm respond mainly to only one whisker (Rhoades et al., 1987; Shosaku, 1985; Waite, 1973). However, Waite (1973) had described how receptive field sizes increased as anaesthetic levels decreased. Studies avoiding deep anaesthesia have revealed that the receptive fields of cells in VPm are larger that originally thought. A cell within VPm will respond to the deflection of a vibrissa that is not represented by the barreloid within which the cell is found (lto, 1988; Simons and Carvell, 1989). These receptive fields are organised into a central receptive field with an excitatory surround. Cells found within a barreloid in VPm respond more strongly and at a slightly shorter latency to the single whisker that the barreloid represents, the principle whisker, its central receptive field. Stimulation of a non-principle whisker in the surround receptive 
field on the other hand elicits a weaker response at a slightly longer latency (ArmstrongJames and Callahan, 1991a; Diamond ef al., 1992a).

To discover if the differing properties of the centre and surround responses to whisker stimulation found in VPm were a product of convergence of afferents from the trigeminal-nuclear complex Rhoades et al. (Rhoades et al., 1987) recorded from cells in the rat VPm following selective lesions of specific trigeminal nuclei. Injections of kainic acid into the principle nucleus of the trigeminal-nuclear complex initially caused cells in VPm to stop responding to whisker stimulation. Over a period of days the cells of VPm started to respond to whisker stimulation but with larger receptive fields. The response properties were "interpolaris like" in there characteristics. The unmasking of response properties in VPm driven by interpolaris input was confirmed when the responses were all but abolished by subsequent injections of kainic acid into the interpolaris subnucleus (Rhoades et al., 1987). The results suggest that the receptive fields found in VPm are the result of a convergence of these two inputs from the trigeminal-nuclear complex. These results were confirmed when Friedberg (1991) found that following a lesion of the principle nucleus cells in VPm responded to several whiskers at long latencies.

\section{Posterior Nucleus (PO)}

PO encloses VPm dorsomedially and caudally. The rostral dorsomedial portion of the nucleus receives topographically organised input from the whole surface of the body including the whiskers. Figure 6 shows that in transverse section the representation of the body surface in PO forms a roughly mirror image of the representation in VPm, reflected about the border between the two subdivisions (Diamond et al., 1992a; Fabri 
and Burton, 1991). Cells found in PO are multipolar, have a varied morphology but tend to have larger dendritic trees than cells found within VPm (Chiaia et al., 1991b).

PO receives a much smaller input from the trigeminal-nuclear complex than VPm. Both the principle nucleus and the interpolaris subnucleus possess populations of cells that project to PO only, and do not send collaterals to VPm. In both cases these populations are fairly small. In the study described above Chiaia et al. (1991a) found that following injections of retrograde tracers in to PO and VPm only $17.4 \%$ of the cells in the interpolaris subnucleus and $7 \%$ of the cells in the principle nucleus were found to project only to PO. With a small population in each nucleus sending projections to both PO and VPm (see above). The magnitude of the input to PO is dwarfed by the massive input that VPm receives from both these nuclei.

The response properties of cells in PO to vibrissal stimulation are different from those found in VPm in almost every modality. Cells in PO respond with less magnitude, at a longer latency, have a lower probability of response to, and are less able to follow a repeated train of stimulation than those in VPm (Diamond ef al., 1992a). It used to be considered that the receptive fields of PO cells were considerably larger than those of VPm (Chiaia et al., 1991b). Ironically, it now seems that when animals are kept under light anaesthesia this is the only response quality in which there is a similarity between cells in the two nuclei. VPm cells on average responding to 4.4 whiskers whereas PO cells typically have an average receptive field of 5.1 whiskers. PO receptive fields do not have a principle whisker or an excitatory-surround receptive field, they respond with near parity to any whisker within their receptive field (Diamond et al., 1992a). 
Although VPm and PO both carry vibrissal information, the nuclei receive afferents from separate populations of cells, receive their main functional input from different trigeminal nuclei, project in different ways in the somatosensory cortex, and carry different qualities of vibrissal information. 


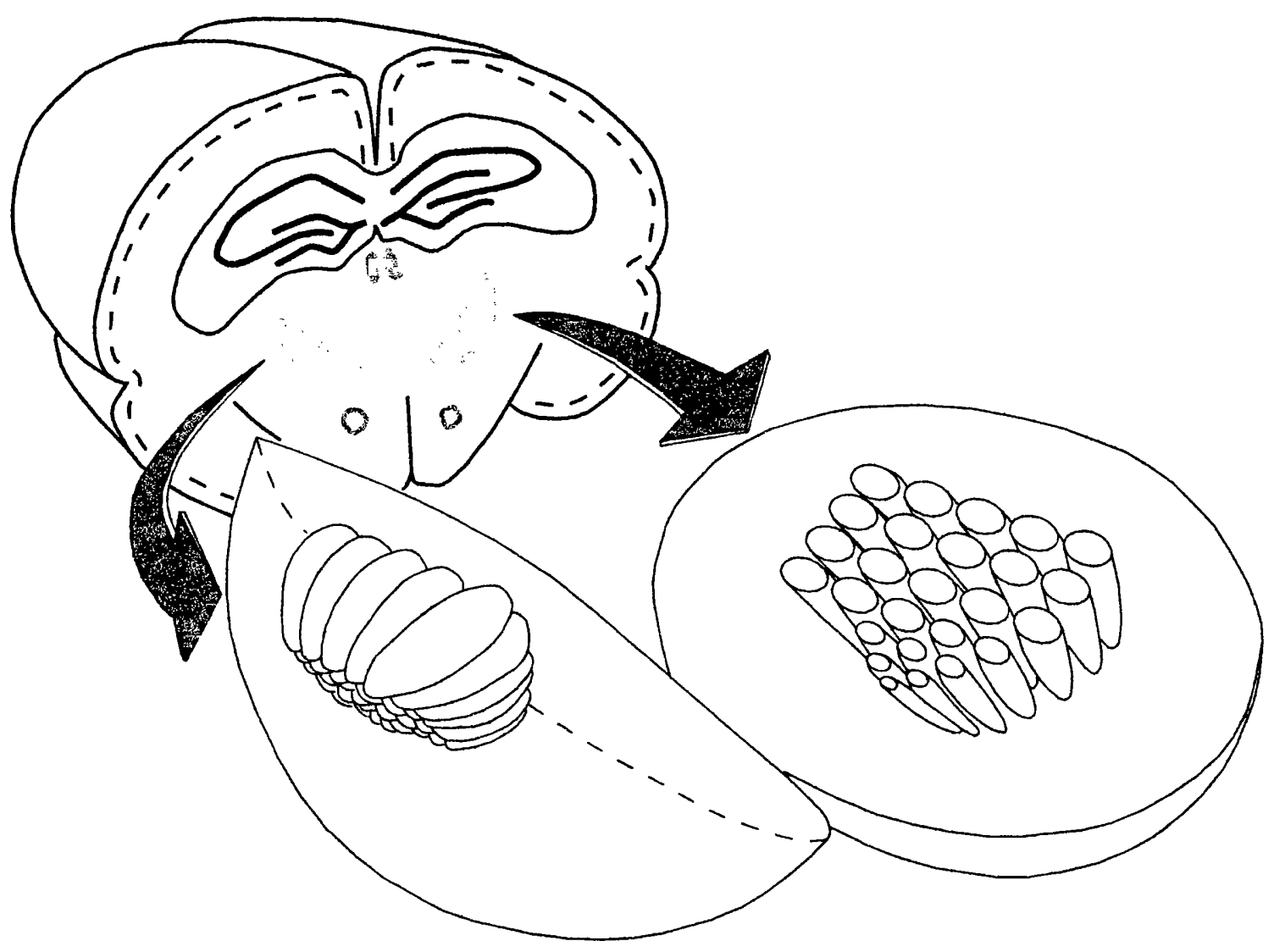

Figure 5:

Diagram demonstrating the three-dimensional relationship of cytochrome oxidase stained barreloids in VPm. Upper diagram shows a rats brain cut coronally at the level of VP thalamic nucleus (shaded semi-circles). The expanded diagrams show the orientation and organisation of the barreloids within the VPm thalamic nucleus. Adapted from Land et al., 1995. 


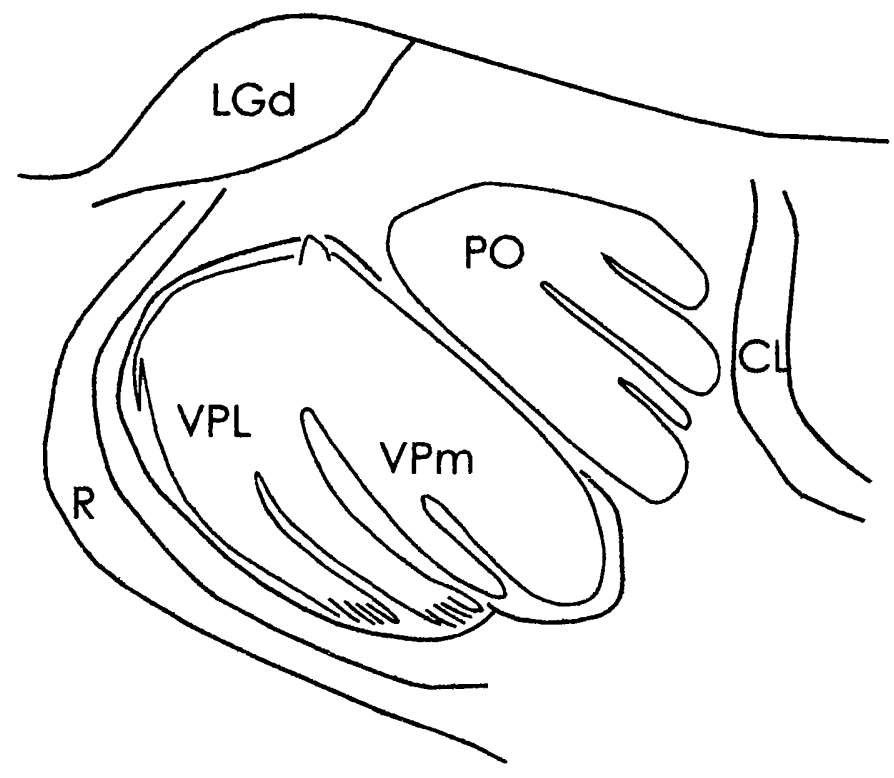

Figure 6:

Schematic drawing to illustrate the somatotopic organisation of Po and VPm. Notice that the two representations are a mirror image of each other if reflected in the border between the two nuclei. Adapted from Fabri and Burton, 1991. 


\section{Somatosensory Cortex In The Rat And Mouse}

In the first half of this century the rat and mouse cortex was the subject of several cytoarchitectonic investigations. Droogleever Fortuyn (1914) and Rose (1912) in the rat, and Lorente de No (Lorente de Nó, 1922) in the mouse identified an area of cortex in which layer IV was densely packed with granule cells. Because of its "koniocortical" appearance the area became known as a sensory-cortical area, although there was no direct evidence at the time that this was somatosensory cortex. Evoked potential mapping techniques allowed experimenters to identify areas of cortex according to the sensory modality which they were responsive to and the body area to which they were sensitive (Marshall et al., 1941). Woolsey (1947; Woolsey, 1952; Woolsey, 1958) used this technique in his exhaustive studies in which he examined the topographic organisation of the somatosensory cortex of a number of mammals including the rabbit, cat, monkey and rat. He found that there were somatotopically organised body maps of these animals in their primary somatosensory cortex (SI). Each of these maps orientated in a similar manner. The representation of the animal's head lying ventrolaterally and facing rostrally. The hind parts of the animal lying dorsomedially near the mid-sagittal fissure. Thus, the representation of the rat, or ratunculus in the primary somatosensory cortex, faces forward, nose, whiskers, perioral region and paws are located rostrally whilst the back and caudal head is found caudally (Figure 7).

\section{Cortical Barrels}


One of the most obvious features of the ratunculus is the massive over representation of the whiskers of the face. By mapping the receptive fields in $\mathrm{SI}$ of the rat Carol Welker (Welker, 1971) delineated the somatotopic organisation of the ratunculus within the primary somatosensory cortex. She found that the head of the rat was represented in $66 \%$ of the total of $\mathrm{Sl}$, almost a third of this head representation was taken up by the mystacial vibrissae. She found that the receptive fields of the neurones in the representation of the head usually consisted of either single mystacial vibrissae or the smaller-sinus hair on the rostral face (see Figure 7).

Woolsey and Van der Loos (1970) had already revealed that within layer IV of the somatosensory cortex in the mouse there were aggregates of cells which resembled barrels by their shape when viewed in tangential section. These barrels are also present in the rat (Welker and Woolsey, 1974). Lorente de Nó (Lorente de Nó, 1922) had observed these aggregates but had no way of identify them as central somatotopic sensory representations of the body surface. These barrels are especially prominent in the area of SI that represents the face. The spatial organisation of these barrels in the cortex mirrored exactly the layout of whisker follicles in the periphery with each whisker represented by a single barrel. This region has been described as the posteromedial barrel subfield (PMBSF) (Woolsey and Van der Loos, 1970)(Figure 7).

In the mouse each barrel is constructed from a cell-sparse centre contained by a celldense wall surrounded by a less dense matrix or septa containing intracortical fibres (Woolsey and Van der Loos, 1970). These typical "hollow barrel" structure is not found in layer IV of the PMBSF of the rat. Instead barrels in the rat are simply cell-dense aggregates or "solid barrels" extending through nearly the whole thickness of layer IV (Welker and Woolsey, 1974). There is a relationship between the size of the barrel and 
the size of the whisker it represents. Larger barrels represent the larger more caudal whiskers. There is also a roughly linear relationship between the number of cells within the barrel (1000-2600) to the number of myelinated fibres in the periphery (70-160) (Lee and Woolsey, 1975). Woolsey and Van der Loos (1970) described barrels after staining for cell bodies with a Nissl stain, but this unique organisation is especially well visualised with histochemical reactions for succinic dehydrogenase or cytochrome oxidase (Killackey et al., 1976; Land and Simons, 1985; Wong-Riley and Welt, 1980). This remarkable central representation of the whiskers in the primary somatosensory cortex has been functionally demonstrated with electrophysiological recording, optical imaging, functional magnetic resonance imaging and the technique for visualising localised metabolic activity, 2 deoxy glucose (Kossut and Hand, 1984; McCasland and Woolsey, 1988; Peterson and Goldreich, 1994; Welker, 1971; Yang et al., 1996).

Rats and mice are not the only animals that have been found to possess barrels. Woolsey and his co-workers (Woolsey et al., 1975) looked for barrels in the brains of 27 mammals. They found barrels only in three of the seven mammalian orders that they examined; rodents, lagomorphs and certain marsupials. They established four principles. i) The spatial organisation of barrels is governed by the organisation of the vibrissae in the periphery. A fact demonstrated very elegantly in neonate and developing mice (Van der Loos et al., 1984; Van der Loos and Woolsey, 1973). ii) Barrels are restricted to layer IV of the cortex. iii) Barrels are not associated with any unique type of behaviour. For example, animals with a well-developed visual system such as the grey squirrel possess barrels. Also, active movement of the whiskers is not essential for an animal to possess barrels. Rats move their whiskers robustly, Guinea pigs do not, and the African porcupine that moves it whiskers at a low frequency of $1 \mathrm{~Hz}$ all posses barrels. iv) Barrels are typically present in smaller mammals (Woolsey et al., 1975). 


\section{Barrel Field Afferents From The Thalamus}

The vibrissal barrels receive two anatomically discrete types of innervation from the two thalamic vibrissal areas (Figure 8). VPm projects most heavily into the barrel centres of layer IV (Killackey, 1973). VPm also projects in a topographic manner to layers above and below the barrels. Terminals from VPm are found most densely in layer IV, this termination extends into layer three, collaterals also terminate in layers $\mathrm{Vb}$ and $\mathrm{VI}$ (Chmielowska et al., 1989; Herkenham, 1980; Jensen and Killackey, 1987; LU and Lin, 1993; Wise and Jones, 1978). In this way the VPm input to the cortex forms columns of terminals. Very few axons from VPm terminate in more than one barrel (Jensen and Killackey, 1987).

PO projects to the barrel field, but the terminals of this projection avoid the barrel centres. Instead PO fibres innervate the septa between the barrels (Chmielowska et al., 1989; Koralek et al., 1988). In this way PO and VPm provide a complimentary projection to the whole barrel field. PO fibres terminate in the septa between the barrels, as well as layers I and Va (Herkenham, 1980; Koralek et al., 1988; Lu and Lin, 1993). No PO terminals are found within the barrel of layer IV (Lu and Lin, 1993).

Barrels contain both smooth and spiny-stellate cells. Both cell types are found more densely packed in the walls of the barrel than its centre. Though this feature is less marked in the rat than the mouse (Welker and Woolsey, 1974). The dendrites of these stellate cells are largely restricted to a single barrel. The dendrites of eccentrically located cells in the barrel tend to point toward its centre. A small number of the cells 
have dendrites that extend into neighbouring barrels (Woolsey et al., 1975). The projections to the barrel centres from VPm of the thalamus terminate on smooth-stellate interneurones and the apical dendrites of large-pyramidal-projection neurones (White, 1976). Layer $\vee$ neurones are arranged preferentially under the periphery of the barrels (Crandall et al., 1986). The apical dendrites of layer Vb neurones tend to pass mainly through the barrel walls (lto, 1992; White, 1976). The septa between the barrels consist mainly of fibres running vertically through the cortex with some similarly orientated dendrites (White, 1976; White and Peters, 1993).

\section{Response Properties in The PMBSF}

Like the cells within the barreloids of VPm, cells within the barrels of layer IV of the primary somatosensory cortex respond best to a single whisker. The whisker, to which a cell best responds is known as its principle whisker, this whisker makes up the cells central receptive field (CRF). The principle whisker of a cell found within a barrel is almost always the whisker which that barrel somatotopically represents, cells will respond to whiskers that surround the principle whisker but less reliably and at a longer latency (Armstrong-James and Fox, 1987). Rapidly and slowly adapting neurones are found in the barrels, and many units display directional selectivity (Simons, 1978). The size of the receptive fields of cells in the PMBSF like those in the thalamus tend to decrease with increasing depth of anaesthesia (Armstrong-James and George, 1988a; ArmstrongJames and George, 1988b). Cells in the supra and infragranular layers in register with a single layer IV barrel form a functional column. Each column has the same principle whisker throughout its depth though cells in different layers respond to the non-principle surround whiskers in different ways. The supragranular layers II \& II respond to movement 
of a few surrounding whiskers, the infragranular layers $V \&$ VI have weaker but larger surrounds (Armstrong-James and Fox, 1987). These functional columns spanning the six layers of cortex can also be visualised with the 2-DG technique (Kossut and Hand, 1984; McCasland and Woolsey, 1988).

Armstrong-James et al. (1991b) showed that the surround response of cells in an individual barrel to stimulation of a neighbouring whisker is generated within the cortex and are not based on collateral input from cells in the VPm which have strong, singlewhisker centre receptive fields. Armstrong-James and colleagues (1991b) made very small lesions in the barrel-field cortex either destroying or partially destroying a single barrel (D2) and its associated septal region. Following these lesions they demonstrated that there was a change in the responsiveness of the cells in the adjacent DI barrel to movement of the D2 whisker. Before the lesion of the D2, cells in the D1 barrel also responded to stimulation of the D2 whisker. After a lesion was placed in the D2 barrel the response of cells in the D1 barrel to the movement of the D2 whisker was reduced. The loss of responsiveness was seen to be directly proportional to the amount of damage done to the D2 barrel centre. The information about the D2 whisker in its surround receptive field cannot have been carried directly to the DI barrel from the thalamus or cells in the D1 barrel would respond to stimulation of the D2 whisker regardless of the integrity of its barrel (Armstrong-James et al., 1991b).

As would be expected from the anatomy of the connections from VPm to PMBSF the earliest cells in a barrel column to be activated by whisker stimulation are in the layer IV barrels. Armstrong-James and colleagues (1992) measured the response latencies of cells within other layers in one barrel column and of cells in surrounding barrel columns 
activated by whisker stimulation. They revealed a pattern of sequential excitation within and between barrel columns.

They found that activation within the column occurs in the following sequence. Cells within layer IV barrels respond first and almost synchronously, cells in layer Vb respond at about 8.5 ms after whisker stimulation. 2 ms later cells in layer III respond, this is followed by layers II and Va which respond 3.5 to $4.0 \mathrm{~ms}$ after the initial response in the layer IV barrel. Finally layer six responds some 4.5 to $5.0 \mathrm{~ms}$ after the cells in the layer IV barrel. Spread of activation to other barrels occurs marginally quicker in the infragranular layers, than in the supragranular layers (Armstrong-James et al., 1992). The strikingly fast response of cells in layer $\mathrm{Vb}$ is consistent with the existence of a direct input to layer $\mathrm{Vb}$ of collaterals from VPm cells projecting to the barrel centres (Keller et al., 1985).

The vibrissae of the rat are exquisitely sensitive vibro-tactile mechanoreceptors. The information they encode from the environment is represented in large areas of the brain. It is obvious from these anatomical and physiological observations that the whiskers are a very important, if not the most important sensory array possessed by the rat. In the next section we will review what is known about how the rat uses its whiskers and what the rat uses its whiskers for in a behavioural context. 


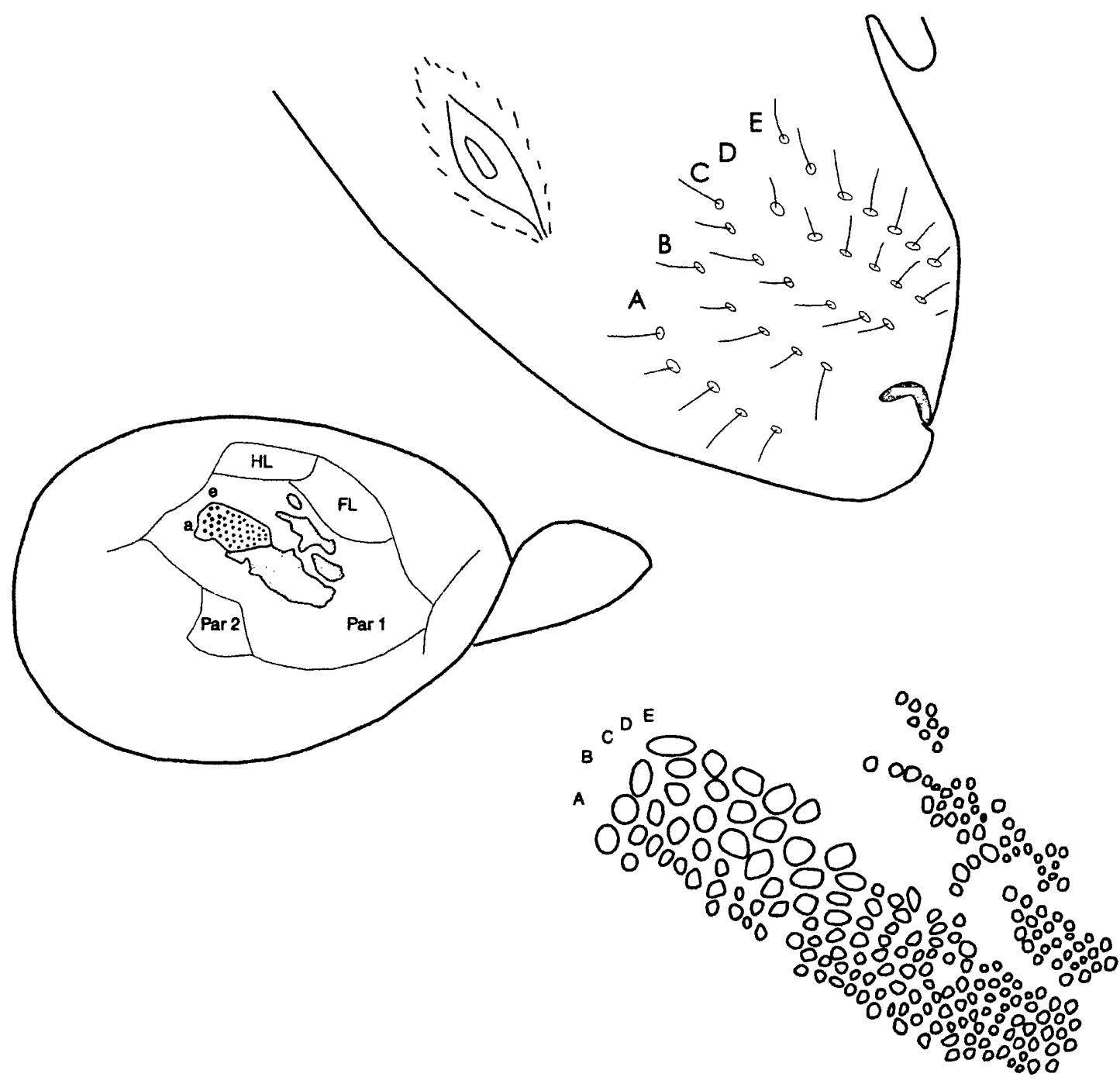

Figure 7:

Somatotopic representation of the mystacial vibrissae in the somatosensory cortex. The barrels of the somatosensory cortex (bottom panel) have a similar topography to the mystacial whiskers on the snout of the rat (top panel). The representation is called the posteromedial barrel subfield (PMBSF). The middle panel shows the position of the PMBSF (heavy dots) within the somatosensory cortex. FL, forelimb area of sensorimotor cortex; HL, hindlimb area of sensorimotor cortex; Par 1, primary somatosensory cortex; Par 2 secondary somatosensory cortex; $O B$, olfactory bulb. The smaller barrels in the bottom panel represent other sinus hairs on the snout, their location is shown by the shaded area in the middle panel. Adapted from Woolsey et al., 1975. 


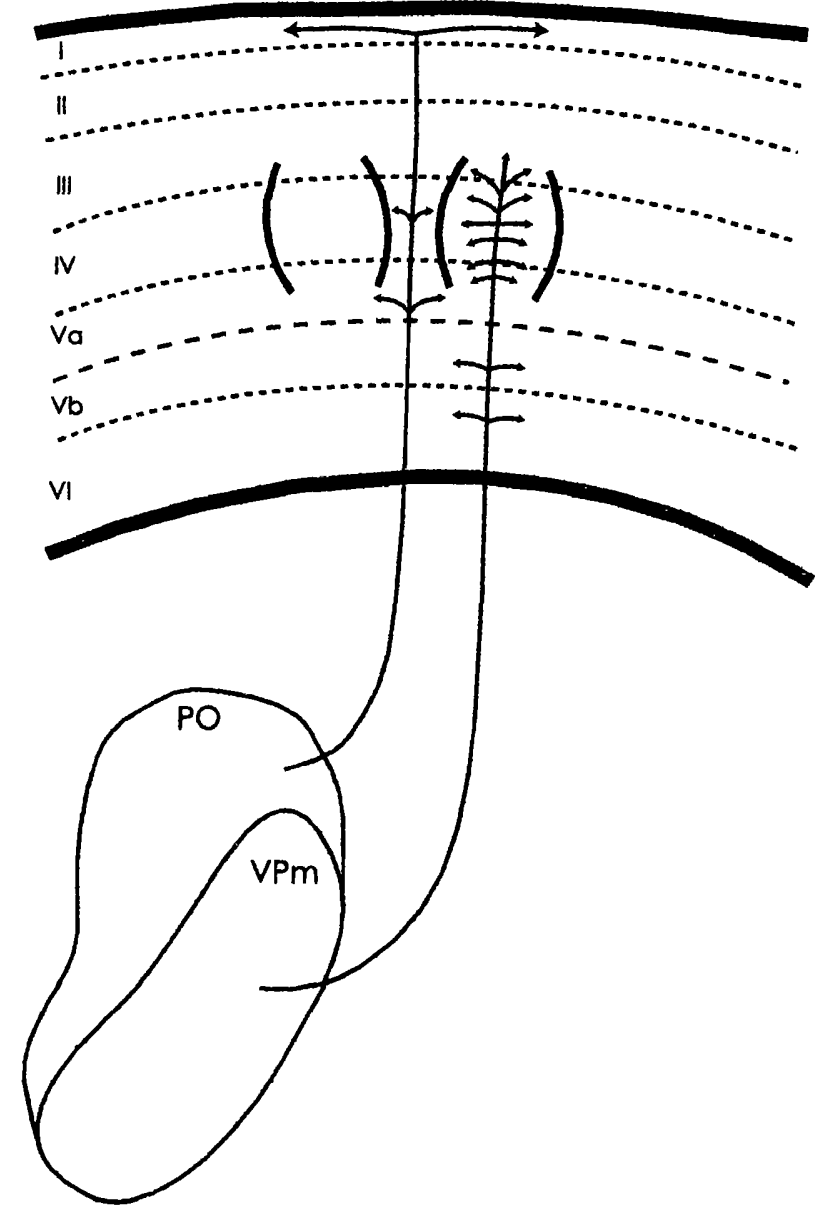

Figure 8:

Diagram showing the projections to the PMBSF form PO and VPm thalamic nuclei. Cortical efferents from VPm terminate in layers III, IV and $\mathrm{Vb}$, particularly in the barrel centres. Inputs from PO terminates I, IV and $\mathrm{Va}$; the projections to layer IV terminate between the barrels. Adapted from Waite and Tracey, 1995. 


\section{What Animals Have Mystacial Vibrissae?}

Animals that possess mystacial vibrissae live in a wide variety of environments and have varied lifestyles. The whiskers are used in a variety of behaviours from exploration to complex social interactions. There seems to be no single evolutionary factor or environmental niche that indicates whether an animal will possess sensory whiskers. Nocturnal and diurnal mammals have whiskers, as do aquatic and non-aquatic mammals.

It is obvious to a casual observer that some animals with mystacial whiskers move their whiskers and some do not. Wineski (Wineski, 1983) divided mammals with mystacial vibrissae into four groups according to amount of mobility displayed by their vibrissae.

- Stationary. Those mammals whose vibrissae only show passive movement. These animals typically have few, small, relatively less organised vibrissae (canids, higher primates, chiropterans, ungulates, edentates and cetaceans).

- Sporadic. Those mammals that do move their vibrissae through wide excurions, but only do so periodically and occasionally. These animals will typically use their whiskers in slow short duration scanning movements. They may have large whiskers but these are usually small relative to the animals overall body size. The vibrissae are normally well organised in a grid pattern (felids, most pinnipeds, certain aquatic fissipeds i.e. sea otters, lower primates, lagomorphs, and larger rodents).

- Rapid. The animals in this category represent typical whisking animals, the rat being one of them. Most are small-nocturnal animals that have moderate or long vibrissae 
in relation to their overall size. The vibrissae are normally found in a highly organised prominent position on the snout. Movement of the whiskers is quick, stereotyped and continuous over long periods of time (rat, gerbil, mouse and hamster).

- Uncertain. In some mammals movements of the vibrissae can be seen but it is unclear whether this movement is the product of specific and independent vibrissae activity or a by product of movement of other specialised, mobile parts of the face. For instance, members of the shrew family have large, elongated, highly mobile snouts. It is unclear whether or not the vibrissal movements observed in these animals are independent, combined with, or a side effect of the animals' whole snout movements. In this way the shrew Sorex palustris and the tree shrew (Tupaia) appear to move there whiskers by way of snout movement only, whereas the Elephant shrew (Elephantulus) seems to use both snout movements and "rodent scanning" (Andrew, 1963). Also the Walrus (Odebenus) the Manatee (Trichechus) and the Dugong (Dugong) all possess large prominent vibrissae. These are located on their large fleshy mobile lips and it is unclear whether the whiskers are moved independently of the lips or not.

It would seem likely that of these groups of mammals, the ones that appear to be most reliant on their whiskers as their major sensory input are those small, typically nocturnal rodents that move their whiskers rapidly. It is this group of animals that have had the mechanics of their whisker movements and their whisker related behaviour most intensively studied. 
What Is whisking?

Rats have very prominent facial whiskers, and characteristically spend a large proportion of their time "sniffing" around their environment. On closer examination the prominent vibrissae on either side of the snout are seen to be in constant motion, protracting and retracting in an anterior-posterior direction. This behaviour was referred to as sweeping by Larsson and Komisaruk (1972) and whisking by Zucker and Welker (1969). Whisking involves very quick movements of a large group of long-fine hairs. To an observer they are discernible only as a blur. These qualities make their movements difficult to study.

Welker (1964) conducted the first empirical study of the movement of the whiskers during exploratory behaviour. Using high speed cameras he filmed the whiskers of rats and reduced the whisking movement during exploration to a repetitive cycle of four components; i) polypnea, ii) protraction and retraction of the mystacial vibrissae, iii) head movements and fixations and iv) protraction and retraction of the tip of the nose. He stated that whisker movements occurred at a rate of between 5 - 11 times per second (Welker, 1964).

Welker investigated the effects of olfactory-bulb ablation, peripheral-nerve section and forebrain removal as well as the effect of depressant and excitant drugs on whisking behaviour. He also studied the emergence of the whisking behaviour in neonate rats.

Depriving the rats of their sense of smell had no effect on whisking behaviour. Sectioning the optic nerve had no effect on the whisking of the animals. The animals in fact increased their exploratory activity after optic nerve section because the brightly lit 
conditions used by Welker for filming had been shown to be aversive to sighted rats (Welker, 1959).

Animals with peripheral-nerve section that removed the somatic-snout afferents including those of the whiskers still continued to whisk. Although they whisked more slowly, the rate was still within the range of the whisking of normal rats. Though these rats exhibited whisking behaviour, they could not locate objects such as food rewards with their whiskers, only when they contacted it with the ventral aspect of their lower jaws or their paws. In rats with bilateral neocortex lesions:

"...clear discrete sniffing movements were seldom seen." (Welker, 1964)

The whisking when present was in short bursts and was of reduced amplitude. It has since been shown that there are no cells in the motor cortex that discharge in phase with the whisking pattern of the vibrissae. Neurones within the motor cortex instead participate in the initiation of whisking bouts (Carvell et al., 1996).

Normal animals treated with an excitant drug (ritalin) showed, on average, an elevated rate of whisking. Those treated with a depressant drug displayed a slightly lower rate. Both groups however remained within the range of whisking for normal rats (Welker, 1964).

Welker's results demonstrate that whisking is a robust behaviour, and not driven by vision or smell. The whiskers do not even have to be sensitive to touch for the rat to produce the behaviour. 
Welker (Welker, 1964) also points out that the whisking seen in 18 day old rats is essentially identical to that seen in adults. Such early emergence and stability suggests that whisking is an innate behaviour not dependent on experience or exploratory behaviour, as rats at 18 days of age have not yet begun to exhibit exploratory behaviour.

Wineski (Wineski, 1983) used a similar method as Welker (Welker, 1964) to examine the movements of the head and whiskers of Golden Hamsters during exploratory behaviour. He identified and examined the movements of three groups of facial vibrissae; i) Mystacial vibrissae of the mystacial pad, ii) supraorbital vibrissae situated above the eyes and iii) the genal vibrissae situated slightly caudal and dorsal to the eye on the cheek. Only the mystacial vibrissae where seen to go through the stereotypical whisking movements. The genal whisker shows very little movement. The supraorbital whisker only moving from a rest position to one of full erection when the animal was alert.

Wineski (1983) observed that the hamsters displayed three types of vibrissal behaviour i) rest ii) alert and iii) active investigation. At rest an animal was seen to be still with its whiskers held motionless at an angle alongside its snout pointing caudally. An alert animal will hold its whiskers erect and away from their face, but the whiskers move little. During active investigation the animal is mobile with its whiskers sweeping through a large arc alongside and in front of its face.

Wineski (1983) noted that during whisking behaviour the hamsters changed the gross position of their whisker by altering the shape of the mystacial pad in which the whiskers are seated. When the whisker are erected in an alert animal the mystacial pad was 
seen to be an even dome in shape. During protraction the pad formed a forwardlyaccented dome, during retraction the doming of the pad was biased towards the rear of the pad. Wineski (1983) stated that during whisking the position of a whisker was the product of the shape of the pad and the tilt of individual vibrissae relative to the pad.

Carvell and Simons (1990) video taped rats whilst they palpated a discriminandum with there whisker tips and described the mechanics of whisker movement in great detail. Carvell and Simons noted that the rats had a dominant whisking frequency of $8 \mathrm{~Hz}$, with a less prominent but consistent peak of $12 \mathrm{~Hz}$. These frequencies were prevalent whether or not the animal was in contact with a target. Whiskers on both sides of the face typically moved in the same direction. Only in $11 \%$ of the video frames in which they studied moving whiskers did they find whiskers on opposite sides of the face moving in different directions. In only $3 \%$ of the frames did they see whiskers on the same side of the face moving in opposite directions. They noted, as had Wineski (1983) with the hamster, that protractions took place at a slower velocity than retractions. Contact with a target decreased the distance the whiskers moved and slowed the whisker movements over the stimuli. The change in velocity was particularly noticeable during retraction, which was halved in speed. The closer the contact was made to the whisker base the greater the decrease. These changes in velocity were the same when the animal contacted a rough or smooth discriminandum (Carvell and Simons, 1990).

They pointed out that the ability to make tactile discrimination in primates with their fingers is enhanced by motion between the fingertip and the discriminandum (DarianSmith, 1984). They proposed that whisking was a strategy to obtain on-going motion between the whisker and discriminandum to enhance the extraction of tactile information (Carvell and Simons, 1990). 
Dorfl (1982) and Wineski (1985) had previously shown in the mouse and hamster respectively that the majority of the follicles of the mystacial vibrissae were contained by a muscle sling. The loop of the sling encompassing a FS-C rostrally and the open end attached caudally. The muscle sling surrounds the lower two thirds of a FS-C so its contraction pulls the base of the vibrissae caudally causing the whisker to pivot about the inner-conical body which is located more superficially. The whisker is therefore caused to protract forward by the contraction of its associated muscle sling. Retractions of the whisker are caused by the natural elastic qualities of the connective tissue that surrounds the follicles. These qualities are consistent with the fact that when an animal is at rest or anaesthetised the whiskers are seen in a "resting", retracted position (Dorfl, 1982). That the velocity and amplitude of whisker retraction is more affected by contact with a surface than protractions also suggests that retraction of the whiskers is a more passive mechanism than protraction which is under a greater degree of muscular control (Carvell and Simons, 1990). Dorfl (1982) commented:

"[the connective tissue] become stretched during protraction of vibrissae; they would contract immediately after the termination of the shortening of the muscle slings: an economical way of executing a stereotyped, fast to-and-fro movement."

To Carvell and Simons (1990) the evidence suggested that a quick, regular, retraction of the whiskers was a "re-setting" movement to prime the whiskers for a forward information gathering sweep, in this way the animal could have almost continuous moving contact with the whisking target, They likened whisker retraction to the saccadic component of the optokinetic reflex.

When the vibrissae passed over the discriminandum they were seen to bend. The bending of the whisker was such that the portion of the whisker contacting the surface 
was not the tip of the whisker but the shaft of the hair. Even relatively straight hairs were seen to contact the stimuli not only at the tip (Carvell and Simons, 1990). They concluded:

"Nethertheless, the data clearly demonstrate that the whiskers do not act as simple rigid levers."

Carvell and Simons (1990) suggested a functional difference between the largercaudal whiskers on the mystacial pad and the smaller more rostral whiskers. Using anatomical and behavioural evidence they distinguished between these two groups of vibrissae on the mystacial pad. As Wineski (1983) and Welker (Welker, 1964) had previously described, they concurred that the larger caudal whiskers moved together as a unit. Two types of muscles are found associated with the mystacial vibrissae, one type of muscle moves the whole mystacial pad the other moves individual F-SCs (described above). The very rostral whiskers of the more ventral rows lack the type of muscle that can move individual vibrissae (Dorfl, 1982). Their observations confirmed Wineski's (Wineski, 1983) report that the large caudal whiskers moved through a large arc whilst the smaller more rostral whiskers remained relatively still. They suggested that as rats palpate an object the smaller rostral whiskers remain relatively still and protracted while the larger caudal whiskers sweep over the discriminandum. They proposed that the animals used their rostral most whiskers to gauge the location of an object and the caudal ones to palpate it (Carvell and Simons, 1990). 


\section{What Do Animals Use Their Whiskers For?}

Whisking is a very complex mechanical behaviour, by which the rat can extract information about Its environment. The type of information that can be discriminated by animals using whiskers has long interested scientists.

Early evidence of the importance of whiskers in cats came in 1823. Broughton (Broughton, 1823) built a maze to resemble the streets of a town out of books. He described how a kitten with eyelids sutured shut would navigate the maze by lowering its head and feeling the floor. In this way the kitten could find its way out of a maze. When Broughton cut off the kitten's whiskers and tested it again he commented:

"The kitten now showed evident signs of having lost the only remaining means of guiding itself. It struck its head repeatedly against the sides of the walls, ran against all the corners, and tumbled over steps placed in its way, instead of avoiding all these as before the removal of the whiskers."

The value of an animal's whisker for navigating in its environment has not only been noted by scientists. In a children's book, "Whiskers", Enid Blyton describes a handsome black cat who after cutting off his whiskers to demonstrate that he will be equally as handsome without them, begins to mis-judge openings and repeatedly gets his head stuck.

Watson (Watson, 1907) found that rats that had been trained to navigate a maze in the light were equally adept at solving the problem in the dark, if not better. The rats were so good at solving the maze in the dark that he worried that rats may have possessed a "supra-human" power of adaptation to the darkness. Tests on blinded rats however, 
demonstrated that they were equally adept at solving the maze as sighted rats. He commented of the blinded rats:

"So far as we could judge, their behaviour was absolutely normal."

In a monograph which is full of lengthy descriptions of rats solving the maze problem, Watson felt that a description of a blind rat in the maze would be so indistinguishable from that of a normal sighted rat that he referred the reader to a previous description of a normal rat.

In investigating the rôle of the vibrissae in the ability of the rats to solve the maze he found that rats trained in navigating a maze became temporarily disorientated and displayed odd motor behaviours if their whiskers were cut. For example, anosmic, normal and blinded animals would walk into walls when their vibrissae were removed. Suprisingly, when the whiskers of normal animals were cut the rats would cling to the floor and would not move. Watson felt that these deficits were transitory, and when he shaved the whiskers of some deaf and normal rats, and left them for 48 hours before testing them in the maze, the animals showed no deficit. He also demonstrated that normal rats without whiskers could solve the maze in the light. Suprisingly he never tested a trained blind animal in the maze after a 48 hour delay following vibrissae cutting. Nor did he have a naïve blind rat without whiskers attempt to solve the maze task. Such experiments would have helped to confirm the rôle of the vibrissae in the navigation of the maze by blind rats.

Richardson (1909) followed up Watson's (Watson, 1907) work by testing blind, anosmic and normal rats on a barrage of behavioural tests. One telling description is of attempting to get a blind rat to jump from one raised platform to another: 
'This rat would not allow his forefeet to leave the platform unless his vibrissae reported some contact with some object. When the platform was beyond the reach of the animal the experimenter touched their tips with a pencil, whereupon he put out his forefeet to step over."

These tests on blind rats reflect a very basic function of the vibrissae; judging distances of objects from the end of the snout.

The most thorough early investigation into the behavioural importance of the vibrissae of the rat was carried out by Stella Vincent (1912) in her monograph "The Functions of the Vibrissae in the Behaviour of the White Rat". In her first experiment she modified Watson's maze by removing the sides and raising the floor. This "elevated runway" was designed to present a more critical task for vibrissal guidance than the walls of a normal maze. On this maze she tested normal rats without vibrissae, normal rats that had vibrissae cut unilaterally, anosmic rats with and without vibrissae, rats with their fifth nerve cut, and blind rats with and without vibrissae.

She found that normal rats with all their mystacial vibrissae cut from one side of their snout followed the edge of the maze that they could feel with the remaining set of whiskers. Ironically this led to rats finishing the maze more rapidly than rats with a complete set of vibrissae that explored the maze in a more random manner. She also performed the experiment that Watson (Watson, 1907) had omitted and tested blind rats with and without vibrissae on the maze without walls. She commented that the blind rats with whiskers:

"seemed to run more freely at first than rats with vision." 
Despite extensive training, over ten times the number of trials that had been required by normal rats to learn the task and five times as many as blind rats with whiskers, blind rats without vibrissae still could not learn to solve the maze. She found that the blind rats reacted very strongly to the removal of their vibrissae:

'They were sluggish, had to be driven to work by blows. When put upon the maze they flattened themselves out, clung sometimes by all four feet to the edges of the walk and refused to budge. It required several sharp raps with a long pencil to start them off and then they went most unwillingly."

Vincent (1912) noted that animals that had all their whiskers cut off or their fifth nerve cut were much more likely to fall off the sides of the maze regardless of the state of their vision than those with their whiskers intact.

After demonstrating the critical rôle of whiskers in blind rats in maze learning, she turned her attention to investigating the rôle of the vibrissae in tactile discrimination. She used a testing box designed so that the rat had a choice of three alleys. The alleys had interchangeable linings, two were smooth and the other corrugated. The animal had to choose the corrugated alley in order to receive a food reward. All the alleys had a copper plate in front of the food box, in the two smooth alleys these plates would deliver an electric shock. The alley linings were changed so that the corrugated alley was never the same for more than two trials. Vincent (1912) demonstrated that vibrissae removal seriously affected the rats ability to learn this discrimination test.

When Vincent (1912) tried to train a rat with cut whiskers on the apparatus it took on average 30 trials more to learn the task. The average time taken was found to be five times as long. The problem with Vincent's (1912) tasks, as would be the problem with the tests of many following investigators, was that the task required of the rats was never 
purely based on vibrissal information. Other senses could always be employed to solve the task. In this case tactile information could be gathered by probing the surroundings with, for instance, the sensitive nose ending. Vincent (1912) cut the infra-orbital branch of the fifth nerve and then tested the animals to show that the whiskers and nose were insensitive. After this the animals were unable to learn the discrimination even after four months of testing, at which point tests indicated that sensation was returning to the affected area and training was discontinued. In her conclusion Vincent (1912) states of the whiskers:

"These hairs then as the whole work shows are delicate tactile organs, which function in equilibrium, locomotion, and the discrimination of surfaces in distinct ways which have been described and the inference is drawn that the great innervation and the fine mobility to which the increased sensitivity of these organs in rodents is due has arisen in compensation for an exceedingly poor vision."

Probably due to the motor adaptations resulting from the removal of the mystacial vibrissae which have already been described, and the general disturbance to an animal of the removal of a major sensory input rats exhibit a general reduction in general activity after whisker removal (Griffiths, 1960; Richter, 1957).

Vibrissal information can be strong enough to drive a rat's behaviour so that it overrides visual information. Sciffman et al. (1970) showed that animals with intact whiskers did not show aversion to the appearance of a "visual" drop, whereas rats with shaved whiskers avoided such stimuli. They hypothesised:

"On visual cliff apparatus the rat's response is mediated primarily by tactile information associated with vibrissae stimulation."

Vibrissal removal has been shown to produce some surprising behavioural consequences. Richter (Richter, 1957) and Griffiths (Griffiths, 1960) stated that when 
they removed the whiskers of domesticated and wild rats many swam less effectively and in some cases drowned. Richter (Richter, 1957) describes one rat:

"[the rat] swam around excitedly on the surface for a very short time, then dove to the bottom, where it began to swim around nosing its way along the glass wall. Without coming to the surface a single time, it died two minutes after entering the tank."

From this description it seems that the removal of the whiskers in this case does not affect the animals ability to swim. Rather the animal is massively disorientated by the loss of its major sensory organ and this leads to this bizarre and fatal behaviour.

William James described that humans with damage to the balance organs of the inner ear feared to swim underwater because of their inability to orientate themselves once they had lost visual cues. Because these people, once underwater, could no longer tell up from down they feared they would not be able to find the water's surface and drown. The disorientation in swimming humans after loss of a major sense organ could be seen as analogous to the cases of rats that drown following whisker removal.

Other more complicated behaviours are affected by whisker removal. Fighting amongst rats can be induced by a footshock. This effect is reduced in sighted or blinded rats if their whiskers are removed (Bugbee and Eichelman, 1972; Flory et al., 1965) or if the whiskers are numbed with lidocaine (Thor, 1975; Thor and Ghiselli, 1973). Whisker removal has been shown to have no effect on spontaneous muricide in rats (Bugbee and Eichelman, 1972; Karli, 1956), though cutting the infraorbital nerve has more recently been reported to reduce muricide significantly /Gregoire and Smith, 1975). 
How Sensitive Are The Whiskers?

Guic-Robles et. al. (1989) pointed out that although certain behaviours i.e. guidance, surface discrimination and other exploratory and social behaviours described above had been shown to be affected by the removal of whiskers or the transection of the trigeminal nerve, none of the tests used to derive these conclusions were pure vibrissal discrimination tests. She noted that the exception were the experiments of Hutson and Masterton (1986) that will be discussed later.

Using a modified Sutherland box (Sutherland and Mackintosh, 1971) Guic-Robles and her colleagues trained rats with eye lids sutured shut to lean across a gap to discriminate between two textures of sandpaper, smooth (200 grains $\mathrm{cm}^{-2}$ ] and rough (25 grains $\mathrm{cm}^{-2}$ ) which they could touch only with their whiskers. They associated the smoother sandpaper with a food reward that the rats could jump across to receive in a goal box. All the rats easily reached the criterion level of $85 \%$ correct choices per session. They then cut the rats whiskers. The rat could be left on the apparatus for up to 45 minutes and it would make no attempt to reach the food reward beyond the landing platform, which was now out of reach of the rat's nose.

In the same study that Carvell and Simons (1990) had examined vibrissal movements during whisking they also used a task similar to that of Guic-Robles et al. (1989) to test the absolute limits of the vibrissal system to distinguish between discriminandum of different roughness. Using plastic cylinders milled with fine grooves they found that the rats could distinguish between a smooth surface and a "rough" surface that had $30 \mu \mathrm{m}$ deep grooves milled $90 \mu \mathrm{m}$ apart for a food reward. They noted that this quality of 
discrimination was comparable to the abilities of primates to discriminate textures with their fingers:

"The discrimination capability of these animals is quite remarkable inasmuch as blindfolded rats are able to perform reliably rough-smooth discriminations that we ourselves found challenging with our fingertips"

They pointed out that because the whiskers in the mystacial pad are continuously regenerating, the whiskers are not always of the same size or dimensions, indeed some follicles will sometimes have two whiskers growing out of them, which would effect the geometry of the whisker field and the stiffness of the whiskers themselves. A number of whiskers will touch an object at any one time for different durations. The evidence suggests that the whiskers are not a system of absolute references, with the whiskers utilised as "feelers".

"It may be useful, therefore, to regard the whisker field in its function [their italics] as a continuous receptive sheet, more akin to glabrous or hairy skin that the punctate anatomical characteristics of the vibrissa system might offhandedly suggest"

They go on to say:

"We are struck not only by the exquisite tactile sensitivity of the rat vibrissal system, but also by the dynamics of whisking behaviour. As described above, the compliment of mystacial vibrissae forms an almost fluid-like mosaic of mechanical sensors extending laterally and rostrally as much as $50 \mathrm{~mm}$ around the animal's head. Whisker movements are rhythmical and rapid, so much so that, viewed in real time, the whisker field appears only as a blur. Viewed in slow motion, it is clear, that within a fraction of a second rats repeatedly scan a surface with their vibrissae and, in so doing, derive information about its texture."

It is clear that the vibrissal system is a complex, highly sensitive sensory system. 


\section{Sensory Guidance Of Movement}

Visually-Guided Jumping As A Model Of The Sensory Guidance Of Movement

Study of visually guided jumping is a useful experimental paradigm to explore how animals use sensory information to guide their movements. An animal in which jumping is a natural behaviour can be easily trained to jump under laboratory conditions. Small laboratory animals such as rats (Russell, 1932) and gerbils (Ellard et al., 1984) will jump accurately over a wide range of distances. Animals typically jump the gap plus a small additional constant. Observations of intact animals whilst manipulating the experimental conditions can tell us what cues are being used to gauge distances. Lesion experiments may tell us what neural substrates underlie the ability to extract these particular qualities from the environment. Jump/no jump decisions by the animals may tell us if the animal can utilise the sensory information being fed into the "system" to produce a coherent motor output.

Small rodents use both dynamic and static cues to accurately estimate jumping distances. Motion cues are one of the most important dynamic cues used in this situation. Rats self generate motion cues by producing a series of vertical head bobs just before they jump. One of the motion cues used by rats to gauge jumping distances is motion parallax. When the rat moves its head, closer objects move over a greater angular distance than objects further away (Ellard et al., 1984). However, motion parallax is not the only motion cue used, as rats make head bobs and can use motion cues to jump accurately over gaps in the absence of parallax cues (Legg and Lambert, 
1990). Head movements are more likely to occur as the gap distance that the animal has to judge increases (Ellard et al., 1984). Such movements are seen in many animals that must make accurate distance judgements. Flying squirrels bob their heads before gliding long distances from tree to tree on enlarged flaps of skin stretched between their front and back legs (Walker, 1968). Before jumping, locusts move their heads from side to side in a behaviour known as peering. They use this movement to extract motion-parallax cues to gauge distance (Sobel, 1990; Wallace, 1959). The fact that locusts display this behaviour suggests that self-induced motion cues are an ancient strategy for solving the problem of extracting distance cues from the environment.

A gerbil with a large lesion of visual cortex $(\mathrm{Ocl})$ will still produce head bobs when preparing to jump. They perform much more variably than sham operated animals in the accuracy of their jumps. The distance by which they clear the gap varies much more and the animals fail to clear the gap in nearly half their trials (Ellard et al., 1986). Although accuracy was severely impaired, the distances jumped by these animals still shows an average relationship to the size of the gap that has to be jumped. Thus, although impaired, the animals still extract visual cues pertaining to the distance to the intended landing point. In addition to motion cues one such cue is retinal image size.

Goodale and his colleagues (1990) trained gerbils on a standard jumping apparatus, they then inserted smaller or larger landing platform in the apparatus. In this way they demonstrated that gerbils use retinal image size cues to determine distances. When they inserted a smaller platform, it's smaller retinal image size made the platform appear to be further away than it really was. In these conditions the gerbil would consistently over jump the gap. With a larger landing platform, the animal would under jump and often miss the platform leading edge. Interestingly, when the smaller landing platform 
was inserted the animal was likely to increase the number of head bobs that it produced as compared to the normal platform (Goodale et al., 1990). The animal produced fewer head bobs for the larger platform. Animals with large Ocl lesions displayed more variable landing positions than sham operated animals. But like normal and sham operated animals, animals with large Ocl lesions were still sensitive to the insertion of larger or smaller "probe" platforms (Carey et al., 1987).

\section{Whisker-Guided Jumping As A Model Of The Sensory Guidance Of Movement}

In an attempt to elucidate the rôle of the barrel cortex in the normal function of the vibrissae of the rat, Hutson and Masterton (Hutson and Masterton, 1986) performed a series of three ablation-behaviour experiments. In their last experiment they presented blind rats with a gap-crossing task. The task is essentially the same as a visual-jumping task, though the animals were prevented from using vision to detect the landing platform by bilateral enucleation. In this experiment the blinded rats had all but the $\mathrm{Cl}$ vibrissae shaved on either side of the snout. The animals were trained on a raised runway split by a gap in the middle. The animals were trained to approach the gap at which point they would have to lean out across the gap to gauge the distance to the other side with there remaining whiskers. At this point the rats would make a jump versus no jump decision to cross to the far platform to gain a food reward, depending on their ability to detect the far platform. Hutson and Masterton (1986) tested animals with ablations of the barrel fields, these animals were tested with both $\mathrm{Cl}$ vibrissae intact, with a single $\mathrm{Cl}$ vibrissae, and with no vibrissae.

They found that blinded but otherwise normal animals with a $\mathrm{Cl}$ vibrissae on both side of the muzzle and blind animals with only one $\mathrm{Cl}$ vibrissae remaining have no trouble 
crossing the gap after detecting the far platform with only their vibrissae. Animals with bilateral barrel field lesions could not cross to the far platform, no matter the state of their whiskers unless the animal could reach to the far side with its nose or paw. The findings that the barrel fields are essential to the animal's ability in the task were reinforced by the results from animals with unilateral barrel field ablations. Such animals with both $\mathrm{Cl}$ whiskers but only one barrel field intact behaved in the same way as normal animals with both $\mathrm{Cl}$ whiskers intact in the task. Animals with only a single $\mathrm{Cl}$ whisker ipsilateral to the barrel field lesion also behaved as normal. However, when animals with unilateral barrel field lesions had the ipsilateral $\mathrm{Cl}$ whisker cut, leaving only the $\mathrm{Cl}$ whisker that had its corresponding barrel destroyed, the animal could only cross the gap if it could reach the far side with their paw or nose. Therefore an animal was capable of the task only if it retained at least one complete vibrissa-barrel system. If the vibrissa-barrel systems were damaged bilaterally then the animal could not complete the task. Interruption either in the periphery, by the cutting of the $\mathrm{Cl}$ whiskers, or centrally by barrel field ablation had the same effect.

They concluded that the ablation of a barrel field was the equivalent to the removal of the corresponding contralateral whisker in the gap-crossing task. The result of the gapcrossing experiment was all the more interesting when one considers the findings of Hutson and Masterton's (Hutson and Masterton, 1986) other experiments reported in the same paper. Using a lick-suppression task they demonstrated that the animals were able to detect the passive movement of their whiskers. Animals with a unilateral lesion encompassing the $\mathrm{Cl}$ barrel field retained normal sensitivity to a passive movement of the contralateral $\mathrm{Cl}$ whisker by a sinusoidally modulated air stream. Rats with total ablation of both barrel cortices also retained a normal sensitivity to the passive movement of their $\mathrm{Cl}$ vibrissae. 
They went on to show that an intact barrel field was not only unnecessary for the simple detection of passive movement of the corresponding vibrissae but also for the detection of a change in the quality of the passive movement of the whisker. In this case a rat was able to detect a change in the frequency of a sinusoidal movement of the $\mathrm{Cl}$ vibrissae after the corresponding barrel field had been destroyed to the same threshold as a normal rat. The results suggest that the barrel cortex is not required to make subtle discriminations of whisker movement, nor for the simple detection of the gross movement of a whisker.

In their discussion Hutson and Masterton (1986) hypothesise that the deficit in the gap crossing task may be due to the fact that the motor response required in each task may be underpinned by different sensory-motor systems. If the gap crossing deficit is the result of a specific "sensory-motor disconnection" the question has to be asked; what possible anatomical pathways exist that link sensory to motor areas of the brain? 


\section{Possible Pathways Underlying The Sensory Guidance Of Movement}

\section{The Discovery Of Functional Specialisation In The Brain}

In the latter part of the nineteenth century there was a revolution in the way scientists thought about the localisation of function in the brain. In their seminal experiment Fritsch and Hitzig (Fritsch and Hitzig, 1870) showed that weak electrical stimulation of a small location in the frontal cortex of the dog caused movements on the contralateral side of the body. They also showed that small lesions in a part of the frontal cortex that had produced limb movement on stimulation would result in the animal being clumsy in the use of that limb. The evidence was not immediately accepted as proof of the existence of a specialised motor area. It was postulated that the deficit might have been caused by loss of sensory mechanisms that were required to guide the movement (Munk, 1896). By the end of the century however, after Schäfer (Schäfer, 1898) had pointed out that an animal with a motor cortex lesion retained feeling in its paralysed limb, the idea of a specialised motor cortex came to be widely accepted.

Munk (Munk, 1881) reported that the unilateral removal of the occipital lobe rendered a monkey hemianopic, i.e. permanently blinded in one visual field. Bilateral removal produced complete blindness. Munk's (Munk, 1881) proposition that the seat of vision in the brain was in the occipital lobe was strengthened when damage in the brains of humans who had become hemianopic following disease or head injury was found to be localised in the calcarine fissure after post mortem examination (Henschen, 1890). It was suggested that the visual area was coextensive with the histologically unique striate 
cortex (Henschen, 1890). The pattern of mapping of the visual fields onto the human brain was started by a Japanese physician Tatsuji Inouye (1909). He mapped the visual field defects of soldiers who had come to have small precise brain lesions of the striate cortex due to wounds from newly introduced high velocity small-calibre rifles in the Russo-Japanese war (Glickstein and Whitteridge, 1987). Inouye (1909) compared patient's visual field defects with the site of damage within the brain and produced a basic plan of how the visual fields are mapped onto the human brain. This work was completed by Holmes and Lister (Holmes and Lister, 1916) in the Great War by analysing soldiers with similar brain injuries.

Once the brain was seen to be made up of functionally specialised compartments, rather than some mysterious whole, it became evident that individual areas must have to communicate with each other in order that the brain function as a unit. Thus, it became essential in order to understand how these areas interacted, to discover the anatomical pathways that connect sensory and motor areas. These anatomical links would be essential in order for any organism to guide its movements using incoming sensory information.

\section{Cortico-Cortical Connections And The Guidance Of Movement}

Early investigators all seemed to accept without questioning that motor and sensory areas of the brain were principally connected by a series of cortico-cortical fibres. It had been known since the times of Hippocrates (460-300 B.C.) that the right side of the brain controlled the left side of the body and vice versa (Thomas, 1910). It therefore seemed obvious that the massive fibre tract between the hemispheres, the corpus 
callosum, mediated communication between the two sides of the brain. These connections were seen as necessary for behaviour such as bi-manual co-ordination, which requires information transfer between the left and right sides of the brain. Therefore it was only natural to believe that the sensory-motor connections within each hemisphere were the cortical $U$ fibres and long association bundles that were easily traceable by the early anatomists in a simple dissection of the brain.

Modern anatomical evidence however, suggests that cortico-cortical fibres do not represent the rapid, direct pathway connecting sensory to motor areas required for rapid, accurate, sensory guidance of movement. For example, anatomical experiments carried out in the mid 1960s demonstrated that the cortical connections between the visual areas and motor areas in primates are very indirect. To connect visual to motor areas the pathway would probably go from striate cortex, through a series of prestriate areas, then to the brain's highest level of visual-motion analysis, the posterior parietal cortex (PPC), area 7. From PPC the pathway continues in the superior longitudinal bundle to the frontal eye fields (area 8 ). From area $8 \mathrm{U}$-fibres run to the premotor cortex (area 6), from there to the motor cortex (area 4) (Kuypers et al., 1965; Pandya and Kuypers, 1969). Such a circuitous route, with so many relays seems counter-intuitive for a system requiring constant, dynamic control.

\section{Experiments Exploring The Function Of The Cortico-Cortical Fibres}

The earliest investigations into the functional nature of the cortico-cortical fibres proved inconclusive. Exner and Paneth (Exner and Paneth, 1889) attempted to isolate the motor cortex by making a deep encircling cut around the cortical area to interrupt any 
cortico-cortical fibres. It was reported that this procedure resulted in animals with motor impairment as severe as a direct motor cortex lesion. These results however, were later difficult to replicate, and the cut they used to isolate the cortex was rather deep and would have probably cut many fibres linking the motor cortex to subcortical structures.

\section{The Split Brain}

The corpus callosum is almost entirely made up of cortico-cortical fibres. The section of this pathway would thus provide an indirect test of the functional rôle of the corticocortical pathway in sensorimotor guidance. Very early studies suggested that cutting the corpus callosum of dogs produced no obvious deficits or symptoms (von Koranyi, 1890). It was still seen the to be the case by later experimenters (Bremer, 1956) provided that no damage is done to any surrounding structures. Hartmann and Trendelenberg (Hartmann and Trendelenberg, 1927) tested monkeys on a variety of behavioural task that require bi-manual co-ordination and found they showed no impairment after callosal section.

In the 1950s and 1960s there was an explosion of knowledge about the transfer of information between the hemispheres and the nature of this information. The majority of this evidence came from the laboratory of Roger Sperry, and for this work he was awarded the Nobel Prize in 1981. Much of this evidence came from behavioural studies of monkeys in which the corpus callosum and optic chiasm had been cut. In such a preparation visual Information received in one cerebral hemisphere could not be transferred to the other hemisphere directly via the callosum. 
Early evidence suggested that the callosum was necessary for the transfer of sensory information between the hemispheres. Myers (1956) demonstrated that a cat with a midline section of the optic chiasm trained monocularly on a visual form discrimination task displayed a high level of performance when the animal was tested using the untrained eye, this is known as interocular transfer. Myers found that section of the corpus callosum prior to training prevents the interocular transfer of the learned task. A similar effect was shown in monkeys (Downer, 1958; Sperry, 1958).

Studies of animals' poor performance after brain lesions are often criticised as being indeterminate in their cause. To get around such objections Glickstein and Sperry (1960) tested for intermanual transfer using reversal training. Normal and callosalsectioned monkeys were trained to select one of two discriminanda on the basis of touch alone. Both groups of animals learned these tasks readily. When they had reached criterion performance they were tested with the same pair of discriminandum using the untrained hand. The formerly positive stimulus now was negative. Normal monkeys showed transfer, initially performing at near $0 \%$ correct. Callosal sectioned monkeys performed at chance. When tested with the originally trained hand with the original positive discriminandum callosum sectioned monkeys performed near $100 \%$ correct normal monkeys at $0 \%$ correct.

The experiment shows that the ability of an animal to transfer visual and tactile information between hemispheres to learn a discrimination task is abolished by callosal section. If such sensory information is carried in the callosum for the learning and memory tasks described above, does callosal section affect the successful transfer of sensory information to motor areas of the other side of the brain in order to guide a successful movement? 
Downer (1959) cut the optic chiasm and corpus callosum in monkeys, thus restricting visual information in each hemisphere to the nasal hemifields. He claimed that if an animal were prevented from using one eye that animal would show a strong preference for the hand contralateral to that occluded eye. The result suggested that the visual information could only be used to guide a movement originating in the motor cortex of the same hemisphere.

The hand preference found by Downer (1959) is however not absolute. Glickstein and Sperry (1963) trained a monkey to pick small food rewards from a rotating disk with either hand. Visual information was then restricted to one hemisphere by cutting the optic tract unilaterally. After optic tract section the animal could use either hand to retrieve the food. The corpus callosum and anterior commisure were then cut. For a few days after surgery the animals were mildly impaired in food retrieval when using the hand ipsilateral to the remaining optic tract. The animal however quickly recovered to preoperative levels in both performance and agility with either hand. If the animal was reliant solely on callosal and commissural fibres to link motor and visual areas on opposite sides of the brain, cutting the callosum and anterior commisure in these monkeys should have rendered an arm "blind" to the contralateral visual field.

Ettlinger and Morton (1963) like Hartmann and Trendelenberg (Hartmann and Trendelenberg, 1927) before, showed that monkeys trained to perform tasks that required bi-manual co-ordination showed no deficit after the forebrain commisures were sectioned. Mark and Sperry (1968) later demonstrated that if the midbrain commisures were cut as well the monkey would display a deficit in bimanual coordination but this deficit also recovered with time. The result suggests that both sides of 
the brain have independent control over both limbs and do not need the callosum to interact with each other. If the brain does not require the cortico-cortical fibres of the collosum for sensory-motor communication between the hemispheres, are the corticocortical fibres necessary for communication within a hemisphere?

Lawrence and Kuypers (1968a; Lawrence and Kuypers, 1968b) demonstrated that severing the pyramidal tract, the main descending pathway from the motor cortex to the spinal cord, in a monkey caused a deficit in independent and apposable finger use in the hand opposite the hemisphere from which the severed tract originated. Kuypers and his colleagues pointed out that although a split brain monkey could use an ipsilateral eye to guide a reaching movement of the arm and hand controlled by a "blind" hemisphere, the animal would never fully regain full finger use. Kuypers and his colleagues suggested that the "blind" hemisphere might guide the ipsilateral limb using descending ipsilateral projections to brainstem motor areas. Brinkman and Kuypers (1972) proposed that each hemisphere controlled proximal and complex movements of the ipsilateral side and also the contralateral extremity. If this were the case then it would be possible for the sensory and motor cortices to communicate by corticocortical connections and guide movements from the ipsilateral motor cortex.

Savaki and colleagues (Savaki et al., 1993; Savaki et al., 1996) had split brain monkeys perform tasks that involved visually guided reaching movements, utilising visual information presented to one eye. The animals had to use the arm contralateral to the "blind" cortex. Whilst the animals performed the tasks they were exposed to 2 deoxyglucose, a metabolic marker that labels areas of high glucose metabolism. If Brinkman and Kuyper's (1972) proposal is correct the motor cortex ipsilateral to the arm used for the reaching movements should be labelled. In contrast the motor cortex contralateral 
to the arm used was labelled. According to Brinkman and Kuypers (1972) this cortex should have effectively been "blind" to the information in the opposite cortex once the corpus collosum and other commisures have been cut. This evidence combined with the earlier findings of Myers and his colleagues (Myers et al., 1962) strongly suggest that it is not the ipsilateral hemisphere that controls the "blind" arm of a split brain monkey in tasks requiring the use of unilateral visual input.

Myers et al. (1962) examined the rôle of the cortico-cortical fibres within the hemispheres in visually guided movement. They made deep cuts in the parietal lobe white matter severing the fibres between the occipital and frontal cortex of monkeys. The cuts interrupted all the possible fibres that could travel from the visual cortex to the motor areas in the frontal lobe. Even after these large fibre lesions, monkeys still retained the ability to direct their limbs skilfully under visual guidance. Moreover, destroying the main target of the occipitoparietal visual association fibres, the periarcuate cortex, does not prevent monkeys from making visually guided movements of their contralateral arm, hand or fingers (Halsband and Passingham, 1982).

Haaxma and Kuypers (Haaxma and Kuypers, 1975) performed a similar experiment by training monkeys to pick food from a disk containing six equally spaced radial slots. They then placed lesions in the white matter of the parietal lobe, in an attempt to sever the cortico-cortical connections between the visual and motor cortices. After the lesion the animals specifically lost the ability to use vision to orientate the wrist and fingers to take food from the slots. It is possible that this deficit is underpinned by the loss of cortico-cortical connections between the visual and sensory cortices. The lesion may have damanged subcortical pathways. The lesions would have interupted fibres between the primary visual cortex and the parietal cortex, from where there are many 
descending fibres to lower motor areas especially to the pontine nuclei. One of the monkeys that had a lesion placed more rostrally in the brain, sparing these connections recovered from its deficits.

The evidence suggests that cortico-cortical fibres between or within the cortical hemispheres are not necessary for an animal to make fast, accurate sensory guided movements. How then does the brain link incoming sensory information with brain structures that control movement? There are two major sub-cortical pathways that do connect sensory cortex to motor areas of the brain. One via the basal ganglia, the other by way of the cerebellum.

\section{The Basal Ganglia}

The basal ganglia in primates are made up of 5 interconnected subcortical nuclei. The striatum, made up of the caudate and putamen which receive the majority of the basal ganglia's afferent connections and the substantia nigra, the globus pallidus and the subthalamic nuclei. The globus pallidus and a division of the substantia nigra (pars reticularis) make up the output nuclei of the basal ganglia.

The primary input to the basal ganglia is from the cerebral cortex and its major output, via the thalamus, is back to those same areas of cortex. Areas that send and receive projections from the basal ganglia are prefrontal, premotor, supplementary motor and motor cortex. Virtually the entire frontal lobe sends and receives projections from the basal ganglia. It appears that there are a series of segregated functional circuits that link the cortex, basal ganglia and thalamus. The circuits are thought to be separate, 
activating distinct areas of basal ganglia and thalamus. Current information suggests that there are at least five separate circuits. Each circuit is thought to be functionally separate receiving projection from, and projecting to related areas of cortex. For example, the "motor" circuit is associated with the precentral motor areas, the "oculomotor" circuit with the frontal and supplementary eyefields, the two "prefrontal" circuits with the dorsolateral prefrontal and orbitofrontal cortex respectively, and the "limbic" circuit on the anterior cingulate and medial orbitofrontal cortex.

Most motor structures of the brain either receive direct input from the spinal cord or send direct connections to it, or both. The basal ganglia have no direct control over movements in this way. It has neither direct efferents nor afferents to the spinal cord. Its motor function is therefore mediated via the frontal cortex.

\section{Motor Disorders Due To Basal Ganglia Dysfunction}

That the basal ganglia are involved in motor control was first noticed in post mortem examination of patients with various motor disorders. The degenerative diseases of the basal ganglia Huntington's and Parkinson's cause severe motor disorders. Vascular accidents that cause damage to the basal ganglia also cause motor dysfunction.

The characteristics of movement disorders related to basal ganglia dysfunction are represented at one extreme as severe hypokinetic disorder, the best known example being Parkinson's disease. At the other end of the spectrum are hyperkinetic disorders such as hemiballismus and Huntington's disease. 
Hypokinetic disorders are often associated with a loss of the ability to initiate a movement (akinesia), and movements that are reduced in velocity and amplitude (bradykinesia). Hypokinetic disorders are also characterised by a rigidity of the muscles and a resting tremor. Conversely hyperkinetic disorders are typified by increased motor activity, mainly in the form of involuntary movements, and reduced muscle resistance to passive movement (hypotonia).

\section{Are The Basal Ganglia Involved In The Sensory Guidance Of Movement}

Although the basal ganglia play a critical rôle in the control of movement, it is unlikely that they play a direct rôle in its sensory guidance. The rather indirect access to the motor pathways described above do not suggest that the basal ganglia are in a position for the ongoing guidance of fast, accurate and dynamic movements using sensory input.

Evidence that the basal ganglia are not essential to the sensory guidance of movement also arises from humans with damaged basal ganglia. Parkinsonian patients have a paucity of normal movement. They are rigid, suffer from a resting tremor, and move with difficulty. However, despite these motor disturbances patients can occasionally produce sudden, rapid and accurate movements (Glickstein and Stein, 1991). Anecdotal evidence suggests that under certain circumstances Parkinsonian patients can produce complex motor behaviours: riding a bike, climbing stairs, catching a ball, jogging, or fielding a football. The common denominator amongst these paradoxical movements is that they appear to be under visual control. Animal studies provide evidence that monkeys can improve their movement performance when their basal 
ganglia has been inactivated if the movements are under visual guidance (Hore et al., 1977; Hore and Vilis, 1980)

\section{The Cerebellum}

One of the largest pathways linking the sensory and motor areas of the mammalian brain is via the cerebellum. The cerebellum receives the great majority of its input in the form of mossy fibres from the pontine nuclei. The pontine nuclei are probably the largest subcortical target of the cerebral hemispheres (Mercier et al., 1990; Mihailoff et al., 1978; Wiesendanger and Wiesendanger, 1982b; Wise and Jones, 1977a). In the monkey the population of neurones in the visual areas that project to the pons is probably greater than the populations that project of the basal ganglia or directly to the frontal cortex (Glickstein et al., 1985).

\section{The Cortico-Pontine Pathway}

There is a difference between species as to which areas of cortex project to the pontine nuclei. In the rat all areas of the cortex project to the pontine nuclei (Legg et al., 1989). If the pontine nuclei of a monkey are unilaterally filled with WGA-HRP about half the cortex is seen to contain labelled cells. Areas 4, 6 and the supplementary motor areas contain many retrogradely labelled cells. Area 8 and the frontal eye fields, cingulate cortex, some somatosensory, visual and auditory areas in the parietal and temporal lobes supply a moderate projection to the pons. Few or no labelled cells are found in 
the striate cortex, inferior temporal cortex, or much of the frontal association cortex. Within the visual cortex of the monkey it seems there is a specific subset of visual areas that project to the pontine nuclei (Glickstein et al., 1980; Glickstein et al., 1985).

The extrastriate cortical visual areas of the monkey have been divided into two streams on the basis of their connectivity, the effect of lesions, and their electrophysiological properties (Ungerleider and Mishkin, 1982). Both streams begin in the primary visual cortex but probably have their origin in separate populations of cells, which in furn receive separate inputs from the retina via the $P$ and $M$ layers of the lateral geniculate nucleus. From the striate cortex one stream projects to the lunate and inferior occipital cortex, from there it continues to the inferior temporal sulcus. Because of its position in the brain this stream has become known as the ventral stream. The other stream is directed to the more dorsal and medial extrastriate visual areas and has been called the dorsal stream. The dorsal stream includes cortex on the prelunate gyrus, both banks of the superior temporal sulcus, adjacent regions of parietal lobe including area 7A, and cortex on the lateral bank of the intraparietal fissure.

The alternative names for the ventral and dorsal streams are the "what" stream and "where" stream respectively. These names were largely derived from the very different effects of lesions in cortical areas associated with each stream on the behaviour of experimental animals. Lesions of the inferior temporal cortex, within the ventral stream, are associated with severe deficits in a monkey's ability in a wide variety of visual discrimination tasks. Pattern and object discrimination are impaired, as well as the ability to discriminate between familiar and novel objects (Dean, 1976; Lansdell, 1968; Milner, 1958). Conversely lesions in visual areas associated with the dorsal stream have very different effects on a monkey's behaviour. A lesion in the posterior parietal cortex 
does not effect a monkey's ability in object discrimination; it will however severely effect the animal's visuo-spatial discrimination. The animal cannot for instance, judge the closer of two identical objects to a visual landmark, and has a deficit in visually-guided movement (Buchbinder et al., 1980; Milner et al., 1977; Pohl, 1973; Ungerleider and Brody, 1977). A similar differential effect of lesions in the dorsal and ventral visual streams is present in humans (Newcombe and Russell, 1969).

Physiological recordings from areas within the dorsal and ventral streams also suggest a dissociation of function. Cells in the inferior temporal cortex are found to be very sensitive to the shape, texture, colour and the stereoscopic depth of a stimulus (Adams, 1997; Desimone et al., 1984; Gross et al., 1972; Zeki, 1978). Cells in the parietal cortex do not possess these properties. Neurones in the parietal cortex are particularly sensitive to the direction of stimulus motion and eye movement (Newsome and Wurtz, 1982; Robinson et al., 1978; Sakata et al., 1983; Zeki, 1974; Zeki, 1980).

It seems that the dorsal or "where" stream is concerned with the dynamics and spatial relationships of objects in the visual field, whereas the ventral or "what" stream is involved in pattern and form recognition. In the monkey the pontine nuclei receives nearly all of its visual input from cells within areas belonging to the dorsal, "where" stream. The ventral, "what" stream appears to send virtually no projections to the pons.

The rat cortex does not display such a degree of specialisation. All of the rat cortex projects to the pontine nuclei (Legg et al., 1989). There is however a specific population of cells in the cortex that project to the pons. The pontine nuclei receives afferents from the PMBSF from cells in layer Vb (Legg et al., 1989; Mercier et al., 1990). These layer Vb cells in the PMBSF respond at very short latencies to whisker stimulation (Armstrong- 
James et al., 1987). This rapid corticopontine pathway could be seen to be analogous to the more complex cortico-pontine pathway from the dorsal stream visual areas of the monkey.

\section{Visual Responses In Pons}

The response properties of the pontine visual cells reflect the type of visual input that they receive. In the rat and monkey dorsolateral areas of pontine nuclei receive fibres from visual areas of cortex (Brodal, 1978; Glickstein et al., 1980; Wiesendanger and Wiesendanger, 1982a), in the cat these visual cortico-pontine terminals are found in a more central location (Brodal, 1972a; Brodal, 1972b; Glickstein et al., 1972). In the cat visually responsive pontine cells have large, directionally selective receptive fields (Baker et al., 1976; Glickstein et al., 1972). They code for a wide range of velocities and directions, but they are relatively unresponsive to the shape or orientation of stimuli.

Gibson and his colleagues (1978) identified cortico-pontine cells in the visual cortex (area 18] of the cat by antidromic stimulation. They demonstrated that the cells that project to the pontine nuclei have properties that are similar to responses of cells in the pontine nuclei. Visually responsive cells in the superficial laminae of the same cortical area that do not project to the pontine nuclei differ from those that do project to the pontine nucleus. The cortico-pontine cells therefore represent a specific population of cells with a characteristic set of response properties (Gibson et al., 1978). Similar visual responses have been reported in the dorsolateral pons of monkeys (Suzuki and Keller, 1984; Thier et al., 1988). 
Interestingly, many of the cells in the dorsolateral pontine nuclei also fire in relation to eye movements. Thus the velocity of a moving target can be coded for by cells in the pontine nuclei responding to the velocity of the target across a stationary eye, or cells in the same locale coding for the velocity of the eye if the target is being followed (Suzuki and Keller, 1984). 


\section{Somatosensory Cortical Efferents}

A specific sub-population of visual cortical cells project to the cerebellum via the pontine nuclei, are there analogous cells in the barrel field of the rat?

The barrel field projects to several subcortical targets as well as to other areas of cortex. These projections arise from pyramidal cells which are distributed throughout layers II through VI (Koralek et al., 1990; Wise and Jones, 1977a). As with other areas of cortex, barrel field neurones that project to different structures within the brain are located in specific layers and sublaminae of the cortex (Mercier ef al., 1990). Neurones that project to subcortical targets exclusively have their cell bodies in the infragranular layers (Wise and Jones, 1977a), whereas cells that project to other cortical areas are found mainly in the supragranular layers II and III as well as the infragranular sublamina Va (Koralek et al., 1990).

\section{Cortico-Thalamic Projections}

VPm and PO receive their main reciprocal projection from the barrel field from pyramidal cells located in layer $\mathrm{VI}$ with an additional input from deep layer $\mathrm{V}$ (Chmielowska et al., 1989; Wise and Jones, 1977a). However, small injections restricted entirely to VPm result in retrogradely labelled cells exclusively located in upper layer VI, the cells of layer Vb projecting only to PO (Good and Killackey, 1991). Efferents to PO and VPm from the barrel field terminate in a somatotopic manner (Fabri and Burton, 1991). In the mouse efferents to and from a single vibrissal barrel column have been 
described as terminating in VPm in an arc in that covers the barreloid representations of an arc of whiskers (Hoogland et al., 1987). More recently however, it has been shown that if injections of tracer are restricted to a single barrel in the PMBSF of a rat retrograde and orthograde label in VPm are restricted exclusively to the corresponding barreloid (Land et al., 1995).

\section{Cortical Influence On The Thalamus.}

The descending projections from the barrel fields to the thalamus have a modulatory affect on both VPm and PO. The amount of influence the cortex holds over each subdivision is dramatically different.

Diamond and his colleagues (1992b) studied the response qualities of cells in VPm and PO to whisker stimulation in great detail. They pointed out that the response latencies of cells in VPm had an interquartile range of $4-6 \mathrm{~ms}$. In the cortical barrels it is $7-10 \mathrm{~ms}$, while in PO it was found to be 12-22 ms. Given these latencies it seemed unlikely that PO was directly involved in triggering the initial responses in the cortical whisker barrels.

PO receives a dense projection from the primary somatosensory cortex (Fabri and Burton, 1991; Hoogland et al., 1987). Quantitative studies of the size of the direct input to PO from the trigeminal complex (Chiaia ef al., 1991a) suggest that it is probably smaller in size than the input it receives from the primary somatosensory cortex. Also, the descending fibres from the cortex to PO form very large glomeruli in which the cortical terminals make repeated contact with the thalamic cells, such synaptic formations are not present in VPm (Hoogland et al., 1987). 
The differences in response latencies combined with the anatomical strength of the connections descending from the cortex to PO brought Diamond and his colleagues (Diamond et al., 1992a) to propose that the vibrissal barrel field cortex is a major influence on the properties of PO. In an experiment in which they inactivated the barrel cortex by either cooling the cortex or application of $\mathrm{Mg}^{2+}$ they found that the cells in PO stopped responding to whisker stimulation following cortical inactivation, while cells in VPm remained unaffected (Diamond et al., 1992a).

They concluded that the rôle of PO was not to influence directly the initial response of somatosensory cortical neurones. Rather, the neurones of PO may influence the horizontal spread of activity between the barrels of the somatosensory cortex. They presented anatomical and electrophysiological evidence in support of this conclusion. First they pointed out that PO projects not directly to the barrels, but above, below and in-between them, an appropriate pattern if the rôle of $P O$ is to influence communication between barrel columns. Secondly they pointed out that the latency of excitation of the cells in the septa between the barrels to whisker stimulation was such that the response of these cells probably reflect a spread of excitation from the barrels themselves, as well as the arrival of stimulation from cells in PO. They suggested that the strength of this simultaneous input from PO would influence the passage of excitation which passes through a chain of intracortical neurones between barrel columns (Diamond et al., 1992a; Diamond et al., 1992b).

If $\mathrm{PO}$ is influential in the spread of excitation through the barrel field, yet is influenced predominantly not by ascending projections from the trigeminal system but by descending fibres from the cortex, there must exist a rapid feedback pathway from the 
cortex. Such a fast feedback from cortex to PO is seen in the response properties of the cortical cells that project to PO. The layer $\mathrm{Vb}$ cells that project to PO respond only slightly after cells in the layer IV barrels due to collaterals from the VPm projection to barrels centres (Armstrong-James and Fox, 1987).

As it does with PO, the barrel field does exert a degree of descending modulation over the response properties of cells in VPm. The modulatory effect is complex (Yuan et al., 1985) and is not the main driving force behind the properties of the receptive fields found in VPm. These are of course a combination of inputs from principle sensory nucleus, and subnucleus interpolaris of the spinal nucleus of the trigeminal-nuclear complex (see above)

\section{Cortico-Pontine Projections}

Neurones of the barrel field that project to the pontine nuclei have their somatas in sublaminae $\mathrm{Vb}$. Wise and Jones (1977a) originally reported that the largest cells found in layer $\mathrm{Vb}$ were the origin of all the descending fibre to the midbrain, medulla, spinal cord and the pontine nuclei. They reported that after injections of free horse-radish peroxidase (HRP) into basilar pons about $10 \%$ of cells in the somatosensory cortex were labelled. They also stated that the labelled cells observed were found in clusters alternating with gaps containing unlabelled cells.

Since this early description Legg et al. (1989) using the more sensitive tracer, horse-radish peroxidase conjugated with wheat-germ agglutinin (WGA-HRP), made large injections which filled the pontine nuclei. After an injection of WGA-HRP into the pontine nuclei 
the retrogradely labelled cells were confined exclusively to sublaminae Vb. Labelled cells filled sublaminae $\mathrm{Vb}$ from its upper to lower border throughout the PMBSF. After an injection that fills the pontine nuclei it is seen that in many sections counterstained with neutral red every identifiable pyramid cell in sublaminae Vb of the PMBSF appeared to be retrogradely labelled (Mercier et al., 1990). These labelled cells were evenly distributed throughout somatosensory layer $\mathrm{Vb}$ without any sign of the clustering described by Wise and Jones (1977a).

The size of the cortical projection to the pons suggest it is an important route for information leaving the cerebral hemispheres. The fact that virtually all the cells of layer $\mathrm{Vb}$ are retrogradely labelled following an injection of WGA-HRP that fills the pons suggests that the other structure that receive innervation from layer $\mathrm{Vb}$ (see above and below) must do so from collateral fibres from neurones sending descending projections to the pons.

Cells in layer $\mathrm{Vb}$ respond at a short latency to whisker movement (Armstrong-James et al., 1987) and project to the pons. The properties of the pathway is similar in character to the rapidly responding, fast conducting pathway found between layer $V$ cells in visual area 18 and the pons of the cat (Gibson et al., 1978), which are probably involved in the visual guidance of movement (Stein and Glickstein, 1992)

\section{Cortico-Striate Projections}

Cortico-striatal cells occur mainly in layer Va (Hersch and White, 1982; Wise and Jones, 1977a). With a smaller population of cells located ventrally, extending into layer $\mathrm{Vb}$ 
(Mercier et al., 1990). Because of the extent of retrogradely labelled cells in layer Vb after a pontine injection, Mercier et al. (1990) suggested that the narrow tier of cells within layer $\mathrm{Vb}$ labelled after an injection in the striatum had to represent a population of cells that projected to both the pontine nuclei and the striatum, this possibility was also indicated by a double labelled study (Mercier, 1989). Donoghue and Kitai (1981) found a small population of cells that do project to both the striatum and the brain stem. Cells in layer Va that project to the striatum are activated at a considerably longer latency to vibrissal movement compared to cells in the barrels proper (layer IV) and cells of $\mathrm{Vb}$ of the barrel columns (Armstrong-James et al., 1992).

\section{Cortico-Tectal Projections}

Cortico-tectal neurones are found mostly in superficial part of sublaminae $\mathrm{Vb}$, although a few cells are found in the deep part of layer $\mathrm{Vb}$, or the deeper parts of sublaminae Va (Kassel, 1982; Mercier, 1989; Wise and Jones, 1977b). Fluorescent double label studies demonstrate that many but not all cells in layer Vb have bifurcating fibres which send projections to both superior colliculus and the pontine nuclei (Mercier, 1989).

\section{Cortico-Trigeminal Projections}

Cortico-trigeminal fibres from the primary somatosensory cortex also arise from pyramidal cells in layer Vb (Wise and Jones, 1977a). These fibres terminate throughout the contralateral trigeminal nuclei especially in the caudal subnucleus of the spinal 
sensory nucleus. The densest areas of termination in the trigeminal-nuclear complex are in the septa that separate the representations of single whiskers (Jacquin and Rhoades, 1990). The populations of cells that project to each individual subnucleus are largely separate, though some cortico-trigeminal neurones project to more than one subnucleus (Killackey et al., 1989). Projections from the primary somatosensory cortex modulate the information being carried between the trigeminal-nuclear complex and sensory thalamus(Woolston et al., 1983).

\section{Cortico-Cortical Fibres}

Cortico-cortical pyramidal neurones project to ipsilateral secondary somatosensory cortex, motor cortex, and to the contralateral cortical hemisphere. These neurones are found in cortical layers II, III, and V (Chmielowska et al., 1989; Welker et al., 1988; White and DeAmicis, 1977) 


\section{The Cortico-pontine Pathway}

Almost every cell in sublaminae $\mathrm{Vb}$ of the somatosensory cortex projects to the pontine nuclei (Legg et al., 1989; Mercier et al., 1990) some directly, some via collaterals of the cortico-spinal, cortico-tectal or cortico-bulbar tract (Baker et al., 1983; Cajal, 1909; Keizer et al., 1987; Ugolini and Kuypers, 1986). The fibres arising from these cells run in the basis pedunculi.

The organisation of the fibres within the cerebral peduncle has interested scientists for over a century. At the turn of the century Déjérine (Déjérine, 1901) studied the brains of humans post mortem who had received previous of injury to the cerebral cortex or its efferent fibres. He ascertained that the fibres within the basis pedunculi originated in the cerebral cortex, that the largest contributor of fibres to the peduncle are cortical areas adjacent to or within the central sulcus, and that fibres from the precentral cortex travel in the medial region of the peduncle. Later studies (Beck, 1950; Marin and Angevine, 1962) revealed a relationship between the position of cells in the cortex with the location of descending fibres in the peduncle in greater detail. Fibres from the prefrontal cortex travel in the most medial portion of the cerebral peduncle and fibres originating in the parietal and temporal lobes travel in the most lateral part of the peduncle.

This basic pattern of organisation is repeated in primates, and seems to be generally true for all mammals, although the literature is not as extensive. Studies have been carried out to identify descending fibres in the peduncle from various areas of the cortex in the ferret (Verhaart and Mechelse, 1954), goat (Haarsten and Verhaart, 1967) and rabbit (Drooglever Fortuijn, 1938). Krieg (Krieg, 1947) used a Marchi stain in the rat 
to follow degenerating fibre within the peduncle following lesion in the cortex. He noted that lesions in different cortical areas produce fibre degeneration within discrete, segregated portions of the peduncle.

Glickstein and his colleagues (1992) used WGA-HRP as an anterograde tracer to identify the relationship between the location of cells in the cortex and the position of their descending fibres within the peduncle of the rat. They made small injections of WGAHRP into a number of areas of cortex. After sectioning the brain in a transverse plane they were able to observe labelled fibres within the cerebral peduncles. They concentrated on a region of the peduncle that is about the midpoint of its rostrocaudal extent in the midbrain, about half way between the junction with the diencephalon and the level of the pontine nuclei. Cortico-fugal fibres at this level within the basis pedunculi of the rat are spatially organised. Fibres originating in the frontal cortex travel in the most ventro-medial portion of the peduncle.

Fibres from the primary somatosensory cortex including the barrels run in a more dorsolateral position in the peduncle than the fibres from the frontal cortex (see Figure 9). Fibres from the more caudal areas of the cortex, occipital, temporal, perirhinal and retrosplenial cortex all run in the most dorsolateral part of the peduncle. They saw no overlap between frontal and parietal cortex, some overlap between the parietal and occipital cortex, and considerable overlap between the occipital and temporal cortex (Glickstein et al., 1992).

The general scheme of the organisation of the fibres within the peduncle is the same in the rat as it is in man and higher primates. Fibres from the frontal lobe travel in the most 
ventromedial portion of the peduncle, fibres from the occipital and temporal lobes run in the most dorsolateral portion, with fibres from the parietal lobe found in-between.

\section{Organisation Of The Cortico-pontine Terminals In The Pontine Nuclei}

The pontine nuclei of the rat are positioned between the levels of the trapezoid body caudally and the interpeduncular nucleus rostrally. In the rat the pontine nuclei receive nearly all its input from neurones in layer Vb spread throughout the cortex (Legg et al., 1989). Although layer $\mathrm{Vb}$ cells throughout the cortex are seen to project to the pontine nucleus there is a bias toward the number of fibres originating in the sensory, motor and visual cortices (Glickstein et al., 1985; Legg et al., 1989; Wiesendanger and Wiesendanger, 1982a). The cortex projects to discrete columns of cells running rostrocaudally within the pontine nuclei (Brodal, 1978). In transverse sections these columns take on the appearance of discrete multiple patches spread through the nuclei (Burne et al., 1978; Mihailoff et al., 1985; Wiesendanger and Wiesendanger, 1982b)

The first study of the termination of cortico-pontine projections was by Nyby and Jansen (1951) in the monkey. They subdivided the pontine nuclei into eight divisions according to their position and cytoarchitecture. They then made lesions in the cortex and using a Marchi stain they followed the tract of the degenerating fibres and identified the areas in the pontine nuclei which contained the degenerated fibre terminals. Although the Marchi technique favours the identification of large diameter fibres, the results they produced are similar to results of more recent experiments using more modern and sensitive techniques (Wiesendanger et al., 1979). There is a rough organisational pattern to the projection from cortex. Rostral areas of cortex project to more ventral and 
medial areas of the pontine nuclei and more caudal areas of cortex project to the more dorsal and lateral areas. So, motor cortex projects to medial pons whilst visual areas of cortex in the occipital lobe project to more lateral areas. Like the organisation of the fibres in the peduncle, cells in intermediate areas of cortex project more centrally in the pons. These general principles of organisation are also true in the rat (Legg et al., 1989; Mihailoff et al., 1985; Wiesendanger and Wiesendanger, 1982a).

\section{Sensorimotor Input To Pons In The Rat}

In the rat areas of cortex that represent the face hind or forelimbs project to a number of discrete longitudinally arranged pontine nuclear clusters. Hindlimb and forelimb sensorimotor cortex project to five longitudinally organised zones. The relationship of projections from motor and sensory representations of the limbs is complicated due to the overlap of these representations in the cortex. The hindlimb fibres terminating more caudally than those from the forelimb (Mihailoff et al., 1978). Areas of the motor cortex representing the face project to areas of rostral pons that also receive limb input, with the exception of a small projection field in ventro-medial peduncular region of rostral pontine nuclei. Conversely the area of somatosensory cortex that represents the face, and includes the whisker barrels projects to a separate group of pontine nuclear clusters. Only slight overlap occurs between sensory face projection sites with any of the projection sites representing terminals from the hindlimb, forelimb sensorimotor cortex or the face motor cortex (Mihailoff et al., 1985). The organisation of the corticopontine projection system is such that a small patch of cerebral cortex can project to several patches in the pons. 


\section{Pontine Efferents}

The pontine nucleus provides the major input to the cerebellum through the middle cerebellar peduncle. Ponto-cerebellar fibres terminate as mossy fibres in the granule cell layer of the cerebellum. Our understanding of ponto-cerebellar system is far from complete and there are differences in the organisation of the system between species. The densest projection for visual cortico-ponto-cerebellar fibres in the monkey is to the dorsal paraflocculus. Fibres also terminate in rostral uvula, paramedian lobule and crus II. The projection is bilateral with a strong bias toward the contralateral hemisphere (Glickstein et al., 1994). The paraflocculus is also a major target of visual-pontocerebellar fibres in the rat (Burne et al., 1978).

Ponto-cerebellar projections display a large degree of convergence and divergence. Convergence is seen when small injections of tracer are made into cerebellar cortex. These injections will produce labelled cells in a number of sites of retrogradely labelled cells in the pontine nuclei (Azizi et al., 1981; Mihailoff et al., 1981). Divergence is seen after small injections of orthograde tracers are made in the pontine nuclei. Anterogradely labelled terminals are found bilaterally at a number of sites in the cerebellum. Studies using double labels have shown that single cells may project to two or more sites within a single hemisphere, or even to both hemispheres (Mihailoff, 1983; Rosina et al., 1980).

In this way sensory information descending from the cortex can cross the midline at both the level of the pons, and again when the pons projects to the cerebellum, regardless of the state of the fore and midbrain commisures. 


\section{Cerebellar Connections With Motor Areas Of The Brain}

The cerebellum projects to the rest of the brain via the cerebellar nuclei. The cerebellar nuclei send both ascending and descending projections. The ascending fibres project to the ventralis latralis (VL) of the thalamus, the red nucleus and the superior colliculus (Asanuma et al., 1983; Stanton, 1980). The main target of the cerebellar projection through VL is the precentral motor cortex (area 4] and a cortical area on the banks of the arcuate sulcus (Area 8) (Strick, 1985). The descending cerebellar projections terminate in the pons, nucleus reticularis tegmentis pontis, inferior olive the midbrain reticular formation and the spinal cord (Batton et al., 1977; Kievit and Kuypers, 1972; Sugimoto et al., 1982).

Thus the cerebellum establishes a link with the motor cortex on both sides of the brain via the thalamus. These connections give the cerebellum direct access to the cells of origin of the corticospinal tract. Connections with the midbrain bring the cerebellum into contact with the cells of origin of many of the brain stem motor pathways, the rubrospinal, tectospinal, the vestibulospinal and the reticulospinal tracts. In this way the cerebellum could exert influence on the many descending motor pathways that guide the head body and limbs

The receptive field qualities and directness of the pathways linking sensory to motor areas of the brain are consistent with the hypothesis that this pathway plays a central rôle in the guidance of movement. 

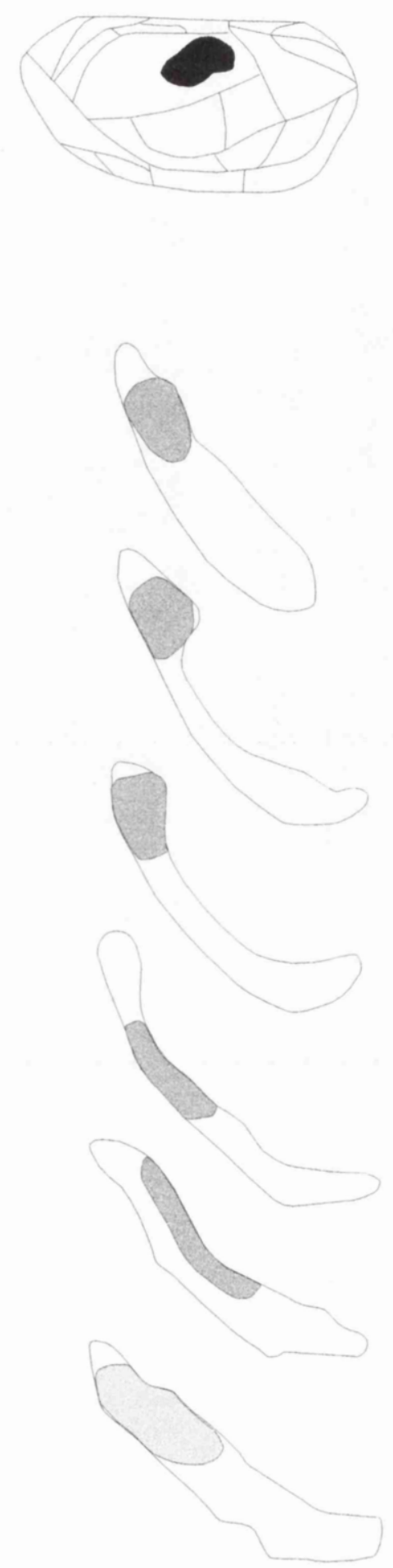

Figure 9:

The lower diagram show the position of labelled fibres in the basis pedunculi following an injection of WGA-HRP into the PMBSF (top panell). Adapted from Glickstein et al. 1992. 
MATERIALS AND METHODS 


\section{Principles Of Experimental Techniques}

Understanding how populations of neurones make connections within the brain is vital to our understanding of how the brain operates. An early method for tracing the connectivity of the brain exploited the degeneration seen in neurones and their axons following damage to the central nervous system. In these degeneration studies a lesion would be made in an area of interest in the central nervous system. Following the lesion the axons of cells that had been located in the area that had been lesioned would die. In this way the degenerated path of the axons would indicate efferent pathways from the lesioned area. These methods were enhanced by the introduction of stains that would specifically label degenerating myelin, although these stains could have the disadvantage of preferentially identifying large, myelinated axons, over smaller nonmyelinated fibres. While the first stains for degenerating axons (Marchi and Algeri, 1885) labelled the breakdown products of myelin, later methods (Nauta and Gygax, 1954) allowed staining the degenerating axons themselves. Retrograde changes in the appearance or death of the cell bodies would identify neurones that had efferent projections that terminated in or had fibres that passed through the lesioned site.

Neurones actively transport substances within the length of their axons in both a retrograde and antrograde direction. Scientists harnessed the cellular mechanism that transport amino acids in an antrograde direction with amino acids labelled with the radioactive element tritium, a neuronal tracing technique known as autoradiograpy. Such labelled amino acids can be injected into the nervous system at a site of interest. At this site the amino acids would be taken up into neuronal somas manufactured into proteins and transported along the axon to the cells axonal terminals. Sections are cut dipped in silver solution and processed like photographic film to reveal the location of the 
labelled cells and fibres. In this way the location of such labelled amino acids within neuronal terminals would indicate the efferent connections of neurones within the site where the labelled amino acids had been injected. Autoradiography proved to be a very sensitive technique. It was in practise however, a difficult and time consuming process to localise the neuronal terminals labelled in this way

Some organic molecules are transported in both a retrograde and antrograde direction and hence they can be used as bi-directional neuronal tracers. Horse-radish peroxidase (HRP) is transported in both an anterograde and retrograde direction and has chemical qualities that allow the molecule to be localised after transport with simple histological procedures. Others organic molecules such as dextran amines and wheat-germ agglutinin (WGA) are transported in both directions but cannot be visualised. In these cases the molecule can be conjugated to another molecule that can be easily visualised, such as HRP, or biotin. In this study two examples of this last kind of tracer have been used, and will be discussed in detail.

The Wheat Germ Agglutinin - Horse Radish Peroxidase (WGA-HRP)

Horse-radish peroxidase (HRP) is a plant glycohaemoprotein, and like many large organic molecules if injected into neural tissue it will be taken into axon terminals by endocytosis and included into cellular vesicles. These vesicles are then transported back to the cell body of the neurone (Kristensson and Olsson, 1971). HRP can be conjugated to the plant lectin wheat-germ agglutinin (WGA). The wheat-germ agglutinin horse-radish peroxidase (WGA-HRP) complex is more likely to be taken into the neurones during what has been termed absorptive endocytosis /Gonatas et al., 
1979). The increased uptake is due to the fact that WGA has a specific affinity for the neuronal membrane molecules $n$-acetyl-D-glucosamine and $\mathrm{N}$-acetylneuraminic acid residues. In this way the WGA-HRP complex has a tendency to bind to the cell membrane thus increasing the likelihood of the molecule being included into an endocytotic vesicle during the process of invagination.

In addition to the increased level of uptake WGA-HRP has several distinct advantages over unconjugated HRP. Free HRP has been shown to be taken up at a greater rate by active neurones compared to neurones displaying lower levels of activity (Singer et al., 1977). WGA-HRP is less influenced by neuronal activity since it is bound specifically to the terminal membrane and transported in a different manner, less affected by activity levels, than the transport of free molecules (Stöckel et al., 1978).

Uptake of WGA-HRP has been shown to occur at low concentrations, due to its ability to bind to the neuronal membrane. Free HRP is taken up actively, but the amount of free HRP that can be taken up by a neurone is proportional to the amount available to the cell. It is therefore regulated by the concentration of HRP in the extracellular space. As WGA-HRP binds specifically to the cell membrane this concentration is much less critical.

WGA-HRP is not taken up by fibres that pass through the injection site, but is taken up by fibres of passage that have become damaged during the injection (Brodal et al., 1983). Conjugated WGA-HRP is less diffusable than free HRP, as this results in the limited spread of tracer from the injection site, making smaller more precisely located injections feasible (Staines et al., 1980) 
HRP is visualised using a histochemical process. When HRP is combined with hydrogen peroxide $\left(\mathrm{H}_{2} \mathrm{O}_{2}\right)$ it forms a HRP- $\mathrm{H}_{2} \mathrm{O}_{2}$ complex. The complex has the ability to oxidise other compounds, such as tetramethylbenzidine (TMB). When TMB is oxidised by the $\mathrm{HRP}-\mathrm{H}_{2} \mathrm{O}_{2}$ complex it produces a dense, dark precipitate. Such a compound that produces a visible redox product is called a chromagen. TMB is very easily oxidised and so is a very sensitive chromogen. A less sensitive chromogen is 3,3'-diaminobenzidine (DAB). In this study $T M B$ is used to visualise transported WGA-HRP, and DAB is used to visualise the extent of WGA-HRP at the injection site. Evidence suggest that DAB gives a more realistic representation of the area of uptake of WGA-HRP (see below).

\section{Biotinylated Dextran-Amines Method}

Dextrans are hydrophilic polysaccharides. They have medium to high molecular weights, are soluble in water and display low toxicity. Dextrans are biologically inert because they possess poly-( $\alpha$-D-1,6-glucose) linkages. These linkages are resistant to cleavage by most endogenous cellular glycosidases. Therefore, dextrans are not easily broken down by catabolic cellular processes. Dextran amines are readily taken up into neurones by endocytosis and are actively transported in both an anterograde and retrograde direction. Dextrans with a molecular weight of up to 70000 daltons have been utilised as neuronal tracers in a wide variety of species.

In this study biotinylated-dextran amines (BDA) with a molecular weight of 10000 daltons were used. Unlike HRP dextrans do not possess inherent chemical characteristics that allow them to be easily localised after they taken up into neuronal tissue. For this reason, the dextrans were conjugated to biotin. Biotin is a molecule that can be localised 
following transport. Biotin has a high affinity to the egg white protein avidin. After fixation the tissue is reacted with avidin that has been conjugated to HRP. The resulting biotinavidin-HRP complex is visualised with the chromogen DAB, in the same redox reaction that is utilised for WGA-HRP. The dextran amines used in this study were covalently linked to lysine residues so that the tracer would bind to surrounding biomolecules during aldehyde fixation.

\section{Cytochrome Oxidase Method}

Cytochrome c oxidase is a transmembrane protein of the inner mitochondrial membrane that is present in all eukaryotes. It is a crucial metabolic enzyme in the electron transfer chain, vital for production of adenosene tri-phosphate (ATP) in the coupled process of oxidative phosphorylation. ATP is metabolised in the brain to fuel fast axonal transport, the construction of macromolecules, and active ion pumping across the cell membrane to maintain resting membrane potential. Enhanced neuronal activity therefore requires an increase in the production of ATP. In this way it is thought that areas of the brain that contain high levels of cytochrome oxidase are "geared up" for levels of prolonged elevated activity (Wong-Riley, 1989). Relative levels of cytochrome oxidase can be visualised in the brain using histochemical methods. Cytochrome oxidase catalyses the oxidation of the chromogen DAB by cytochrome $c$ into an insoluble brown indamine polymer that is detectable under the light microscope. Therefore areas containing high concentrations of cytochrome oxidase will stain darker than areas containing lower concentrations when tissue is incubated in a suitable reaction medium with DAB. 
In this way the vibrissae barrels in the PMBSF previously identified by their cytoarchitecture can be identified with this endogenous metabolic marker. There are however some differences in the patterns of barrel revealed by these methods. Cytochrome oxidase staining does not evenly fill the whisker barrels of PMBSF in the rat. The activity is associated more with the barrel core, as a consequence the pattern of individual barrels revealed appears less squashed together than the pattern that is associated with their cytoarchitecture. Some other features of the cortex are revealed with the cytochrome oxidase method that are not apparent from its cytoarchitecture. Cytochrome oxidase is seen to be in higher concentration in layer $\mathrm{Vb}$ in register with the cytoarchitectonically identified barrels in layer IV and III above it.

The cytochrome oxidase method reveals barrels in subcortical structures which are not apparent from their cytoarchitecture. Barrelettes are found in at least three of the four trigeminal sensory nuclei (Belford and Killackey, 1979), and barreloids are located in VPM of the thalamus using cytochrome oxidase histochemistry (Van der Loos, 1976). 


\section{Behavioural Studies}

\section{$\underline{\text { Subjects }}$}

8 male hooded rats weighing between 375 and 415 grams were used.

During testing and training each animals was placed on a restricted diet. The weight of the animals was not allowed to go below $80 \%$ of their previously determined ad lib feeding weight. Food was given once a day after testing and water was available ad lib. The animals were housed individually in a colony room, on a 12:12h light-dark schedule.

\section{Apparatus}

The apparatus consisted of two tabletops seated on a metal frame table $100 \mathrm{~cm}$ high 30 $\mathrm{cm}$ deep and $125 \mathrm{~cm}$ long. Both tabletops were built so they fitted flush onto the top of the metal frame table. The tabletops were constructed of $2 \mathrm{~cm}$ thick wood, and could be separated by a gap in the middle. The tabletop at one end was fastened securely to the metal frame table. Down the centre of this tabletop an alley was constructed $51 \mathrm{~cm}$ long and $11 \mathrm{~cm}$ wide, the far end of the alley was a dead end. The alley was constructed of $0.5 \mathrm{~cm}$ thick black perspex, and was $18 \mathrm{~cm}$ high. At the dead end of the alley there was a small $3 \mathrm{~cm}$ by $3 \mathrm{~cm}$ concave metal shelf situated $4 \mathrm{~cm}$ above the floor. A $1 \mathrm{~cm}$ hole was drilled in the wall directly above the shelf. Through this hole a food pellet was dropped when the switch of an automatic food hopper was manually closed. 
The other tabletop was not fastened onto the metal frame base but allowed to slide lengthways to allow the gap between the tabletops to be varied. The sliding tabletop was prevented from moving laterally by metal retaining plates built up from the side of the metal frame table. An alley was also constructed down the middle of this table top. The alleys were the same height, but at $19 \mathrm{~cm}$ this alley was wider than the alley on the fixed tabletop and at $71 \mathrm{~cm}$ was also longer. At the far end of the sliding tabletop was the starting box $23 \mathrm{~cm}$ by $10 \mathrm{~cm}$ by $18 \mathrm{~cm}$ which had a hinged roof. A housing in the roof contained a small torch bulb facing into the box separated from the interior of by a glass

port hole. The box fitted neatly into the alley by some flanges extending from the sides of the box. The box was not fixed to the alley and could be moved. A spring loaded door gave access to the runway, on the release of a lever the door would spring upwards into its housing allowing the animal into the alley.

\section{The Testing Room}

The testing was performed in either in a small quiet basement room, or a small soundproofed testing room.

\section{Dark Imaging Equipment}

During testing in the dark all the animals were observed using "night vision". infra red equipment. The testing room was illuminated with infra-red light using a infra-red diode array flood light (GTE). 
It has long been known that the great majority of photoreceptors on the rats retina are rods, although a few scattered cones can be found (Wall, 1934). The population of cones is very small making an estimated $0.85 \%$ of the total number of photoreceptors (Szel and Rohlich, 1992). Despite the paucity of cones, the rat's retina is capable of functioning under both photopic and scotopic conditions (Cicerone, 1976).

Early studies utilising electroretinograms (ERG) and behavioural techniques suggested the existence of a single phototopic spectral mechanism that had a peak sensitivity around $510 \mathrm{~nm}$ (Neitz and Jacobs, 1986). Later, using antibodies specific to cones Szel and Rohlich described two types of cones in the rat retina. This second population was minute, comprising only $7 \%$ of all cones, and therefore only $0.05 \%$ of all photoreceptors. The discrepancy between the single spectral peak and number of cones types was solved when Jacobs and his co-workers using ERGs and behavioural techniques described a second photopic peak in the spectral sensitivity of the rat's retina in the ultraviolet range at around $370 \mathrm{~nm}$ (Jacobs et al., 1991b).

Thus, the rat has a dichromatic retina with peak sensitivities around $370 \mathrm{~nm}$ and $510 \mathrm{~nm}$. In our experiment the rat was held in a brightly lit box before being released onto a runway illuminated with infra-red light $(880 \mathrm{~nm}+/-25 \mathrm{~nm})$. Light adaptation would have taken place before the animal started each trial, and so was using its photopic visual system. At a wavelength of around $880 \mathrm{~nm}$ the illumination was well outside the spectral sensitivity of the longer end of the rat's photopic system, which becomes insensitive as the wavelength increases above $600 \mathrm{~nm}$ (Jacobs et al., 1991a; Jacobs et al., 1991b). 
Neither I nor the rat could see the apparatus without an infra-red viewer. Even after long periods of dark adaptation whilst working with the animals for extensive periods of time in the darkened training room I was never able to see anything without using the infra-red imager. If it was not possible for a thoroughly dark-adapted human to see in the training room it would be very unlikely that a light adapted rat would be able to see under the infra-red illumination. 


\section{Training}

Rats chosen for training were handled and hand fed food pellets of the type to be used as a food reward during training (50 mg precision dust-free pellets, Camden Instruments). The animals were handled regularly for several weeks before any attempt to train them was made. The purpose of the training was to get the rats to jump across a gap using vision in the light to guide their movements, and to cross a gap in the dark using only their whiskers.

The rats were introduced to the apparatus before they were put onto a restricted diet. The first day after the introduction of the restricted diet the animals were placed in the apparatus. The gap between the platforms was closed. A number of reward food pellets were scattered onto the platform on which the animal would be fed after successfully crossing so the rat would associate that end of the apparatus with the food reward. Over the following few days the pellets were placed increasingly closer to the reward shelf, until the animal was feeding from the shelf only.

When the rat was taking food regularly from the reward shelf it was introduced into the starting box. Whilst the rat remained in the starting box the light in the box roof was switched on. In this way the animal's eyes would be prevented from adapting to the dark conditions during the dark trials. The box light was turned off a moment before the box door was opened to allow the animal access to the runway. Once the animal was habitually leaving the start box and going straight to the food reward a gap of $2 \mathrm{~cm}$ was opened between the two platforms. If the rat left the start box and crossed the gap to the reward on three successive trials the gap was increased a further $2 \mathrm{~cm}$. If the animal left the start box but did not cross the gap on two successive turns the gap was 
decreased by $4 \mathrm{~cm}$, or closed completely if the gap was already at $4 \mathrm{~cm}$ or less. Using this sequence the rat soon learned to jump accurately across gaps well beyond the reach of its whiskers, using vision to gauge the distance to jump.

It was important to first train the rats to jump long distances in the light before introducing them to the task of crossing in the dark. If they began their training in the dark they would tend to rely solely on their whiskers to judge the gap distance in both the light and dark. If this happened it became very difficult to encourage the rat to jump further than it could feel with its whiskers, perhaps a suggestion of the overriding reliance the rat has on their whiskers for guiding movement.

During the training in dark the rat was watched using an infra-red night scope. Watching the animals in the dark was important in two respects. To ensure the animals were only gauging the distance to the landing platform with their whiskers and not using their nose, or reaching with their paw. Also occasionally an animal would "maxi-jump". When this occurred the rat would reach the gap and then simply hurl itself as far as it could in an effort to reach the landing platform.

Animals were regularly trained by gradually increasing the length of the gap to cross 16 $\mathrm{cm}$ in the dark, a distance the could only reach with their whiskers. One animal was trained to $17 \mathrm{~cm}$ in the dark, and one to $15 \mathrm{~cm}$, but $16 \mathrm{~cm}$ was the normal distance for an adult rat. In the light the animals would jump up to $34 \mathrm{~cm}$, this was the maximum distance the gap could be opened to due to the size of the testing room.

When an animal became proficient at crossing in the light and dark the animals would be considered ready for scoring. Each trial day the rat would receive a sequence of 50 
individual gap crossing tests, 25 in the light, 25 in the dark. The light, dark sequence of the trials was alternated each day. The starting distance in the dark was $4 \mathrm{~cm}$ below the maximum distance crossed in the dark condition the previous day, the light starting distance was $18 \mathrm{~cm}$, a gap larger than any rat could reach across using its whiskers. The same sequence of $2 \mathrm{~cm}$ increases for three successful crosses and a $4 \mathrm{~cm}$ decrease for two failures was used.

The animals were trained to perform in a stable manner. Performance was considered to be stable if the rat had scored the same record for three days in a row. The animal had to be using only its whiskers to judge the distance in the dark, normally $16 \mathrm{~cm}$, and typically jumping across a considerably larger gap in the light.

When performance first stabilised the rat was ready for a cerebral peduncle lesion. After surgery the rats were allowed to recover on ad lib food until considered well enough for training (usually less than 14 days). After recovery training commenced in a similar manner as before until the rats achieved its pre-operative stable level. The rats were then considered ready for whisker cutting. Two normal-control animals that had not undergone any surgical procedures were trained to the same stable criterion and then underwent similar whisker cutting as the experimental animals.

As a precaution that the surgery performed on the rats had not caused their vibrissae to become insensate a simple behavioural test was performed on some of the rats. Small food pellets as used in the other behavioural testing were held in a pair of forceps. In the dark the forceps were brought into contact with parts of the body surface and the whiskers. The test is normally used to test orientation-localisation behaviours and are normally subjectively scored for their accuracy and speed of response (see Glassman, 
1994). The small reward pellets were eaten very quickly, which allowed me to present several trials in rapid succession. In this way it was easy to make comparisons of the animals response to stimulation of the whiskers and other body parts, and importantly alternating testing between the whiskers on the right and left sides.

As the task was simply designed to ascertain whether the animals could respond to passive stimulation of the whiskers we did not score the tests. The animals were simply observed pre and post-operatively to establish if the animals had similar responses under both conditions. Most conclusively the animals were observed in their responses to quick stimulation to the left and right sides of their bodies post-operatively. As the lesions were always placed on the right side of the brain any effect would be seen as a unilateral loss of the ability to respond to the stimulus, which would be obvious when comparing between the "normal" and "lesioned" sides. 

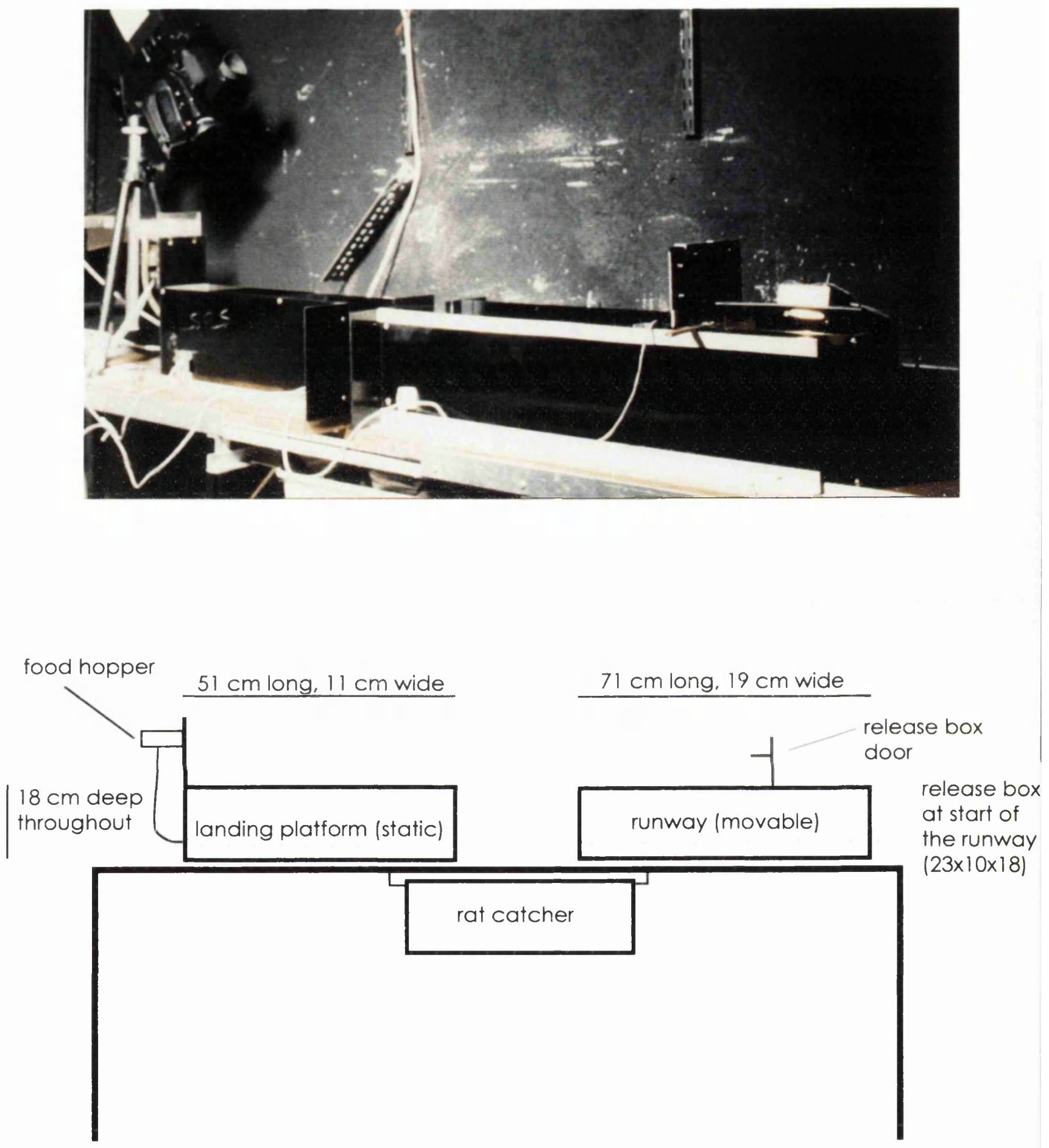

Figure 10:

Top. Photograph of the training apparatus in the training room.

Bottom. Schematic diagram of the training apparatus. 


\section{Surgical Procedures}

\section{$\underline{\text { Surgical Anaesthesia }}$}

Surgical anaesthesia was induced and maintained using a fluothane (ICI) - oxygen mix (2 L min-1, $1.5-2.5 \%$ fluothane) in all cases.

\section{Stereotaxic Procedures}

The stereotaxic co-ordinates for the cerebral peduncle lesion were calculated from Paxinos and Watson's stereotaxic atlas (Paxinos and Watson, 1982). The co-ordinates for the pontine nuclei injections were calculated from Pellegrino et al.'s stereotaxic atlas (Pellegrino et al., 1979). All co-ordinates were made using ear-bar zero as a reference

point. Therefore before all stereotaxic procedures using the lesion probe or injection pipette the instrument tip was referenced to an ear-bar zero from which the stereotaxic measurements would be made.

\section{Cerebral Peduncle Lesion}

The cerebral peduncle lesions were made with a radio-frequency probe (Radionics, Type TCZ) linked to a radio-frequency generator (Radionics, model RFG-4A). The generator 
applies a controlled radio-frequency to heat the tip of the probe. The probe monitors the temperature at the tip which is displayed on the lesion generator.

Six rats received cerebral peduncle lesions. All rats received lesions on the right-hand side. The lesions were made at pre-defined stereotaxic co-ordinates. The animal were placed in a Kopf small animal stereotaxic apparatus (Kopf Surgical Instruments) with its head in a flat-skull position, the incisor bar $3.3 \mathrm{~mm}$ below the interaural line (Paxinos and Watson, 1982). An incision was made in the skin on the midline on the dorsal surface of the skull. The edges of the incision held back with a retractor and the soft tissue scraped laterally to expose the bone of the skull. The skull markers were measured and the skull midline found using the apex of the beta skull marker. The skull midline was compared to the ear bar zero reference midline to assure that they were in register.

The probe was positioned at the appropriate co-ordinates over the skull and the skull marked with a pencil in that position. The skull was thinned at this point using a smalldental drill and bit. The remaining bone was picked off with a dental probe to reveal the surface of the dura. The lesion probe was lowered in a stereotaxic plane until it touched the brains surface. The dorsoventral stereotaxic co-ordinate at the position where the probe touched the brain surface was noted. The probe was retracted and a small cut was made in the dura to allow the electrode to be lowered into the brain. When the electrode was in position the temperature at the tip of the probe was raised to an appropriate point to cause a lesion.

The exact stereotaxic co-ordinate for each case are given in the appendix (page 219), along with the duration and temperature used to create the lesion. 
Injection Of Neuroanatomical Tracer WGA-HRP Into The Pontine Nuclei

In certain cases in order to determine the cortical origin of the cortico-pontine fibres destroyed in the cerebral peduncle, WGA-HRP was injected in sufficient quantity to fill the entire pontine nuclei. To avoid accidentally depositing WGA-HRP via the pipette tract in an area that would result in retrogradely-labelled cells in the cortex that had not originated in the pons, the injection cannula was angled towards the pons through the cerebellum at an angle of $55^{\circ}$ from the vertical. The animals head was angled upwards, the incisor bar $5 \mathrm{~mm}$ above the interaural line to match the Pellagrino et al. atlas from which the co-ordinates were calculated (Pellegrino et al., 1979)

An incision was made in the skin over the caudal skull and neck. The soft tissue was retracted and the muscle covering the caudal skull cut in the midline and retracted. The bone of the skull was then removed either by ronguering up from the level of the foramen magnum or by drilling a hole at the midline and expanding the opening until the required size was achieved. Due to the large number of widely spaced injections the opening of the skull caudal to the cerebellum was approximately about $4 \mathrm{~mm}$ by $4 \mathrm{~mm}$.

Eight separate injections of WGA-HRP were used to fill the pons completely. These injections were made at two levels in the antero-postero axis, at $1.0 \mathrm{~mm}$ and $1.5 \mathrm{~mm}$ anterior to ear bar zero. At each of these levels two injections were made on either side of the midline. One $0.8 \mathrm{~mm}$ the other $1.8 \mathrm{~mm}$ from the midline. All the injections were made at a position $0.3 \mathrm{~mm}$ below ear bar zero. 
The tracer was pressure injected through a glass pipette with its tip pulled into a fine point. A picospritzer (General Valve Company) was used to apply timed bursts of pressure to the open end of the pipette. The quantity of tracer injected was monitored by observing the movement of the meniscus of the WGA-HRP within the pipette barrel through a telescope with a calibrated graticule (Gibson et al., 1984). $50 \mathrm{nl}$ was injected at each of the eight injection sites. If the pipette became blocked during an injection it was removed from the brain and the tip rinsed in saline, during rinsing pulses of pressure were applied to the pipette to blow out any substance blocking the pipette tip. When the tip unblocked WGA-HRP could be seen leaving the tip under an operating microscope. To minimalise the tracer spreading back up the injection track the pipette was left in place at the injection site for at least five minutes after an injection was finished before it was removed. Surgery was completed in a similar fashion to that used for the cerebral peduncle surgery. The volume and co-ordinates of the injections are given in the appendix (page 219).

\section{Injection Of Neuroanatomical Tracers Into The PMBSF}

The neuronal tracers BDA and WGA-HRP were injected into the vibrissal barrel fields of one animal in each case. The injections were made at co-ordinates calculated from the Paxinos and Watson (1982) sterotaxic atlas. Both tracers were injected under pressure using the same method as used to inject WGA-HRP into the pontine nuclei. The animals were held in the stereotaxic equipment in a flat plane skull position (Paxinos and Watson, 1982). The skull was exposed as for the cerebral peduncle lesions. The injection pipette was placed at the required co-ordinates over the skull, the skull was marked. The skull was then thinned at this position, the remaining bone removed with a dental probe to expose 
the brain. The dura was then cut to allow the entry of the injection pipette. The injections were made $1 \mathrm{~mm}$ under the surface of the brain. The surgery was finished in a similar procedure to that used in surgery of the cerebral peduncle. The volume and coordinates of the injections are given in the appendix (page 220).

\section{Whisker Removal}

The animals were lightly anaesthetised and the whiskers were cut close to the base with fine scissors. The follicles were never damaged during this procedure. In some cases the whiskers ipsilateral to the peduncle lesion were cut; in other cases it was the contralateral whiskers. In order to confirm the rôle of whiskers in gauging the gap they were shaved unilaterally and bilaterally in 2 normal rats that had been previously trained to criterion on the apparatus. Cutting the whiskers was used rather than plucking as the later may cause damage to the delicately innervated F-SC. Whiskers will sometimes grow back

withered and corkscrewed after plucking (personal observation; personal communication, Ebner 1998) which does not occur following cutting the whiskers. 


\section{Perfusion}

\section{Perfusion Of WGA-HRP Injected Animals}

Following a survival time of 48 hours the WGA-HRP injected animals were deeply anaesthetised and then killed by asphyxia with $\mathrm{CO}_{2}$. When the animals had stopped breathing the thorax was opened and a perfusion needle was placed into the left ventricle or directly into the aorta. The right atrium was then cut. Blood was initially washed out with $1000 \mathrm{ml}$ of $0.9 \% \mathrm{NaCl}$, containing 1000 units of heparin. To fix the tissue the animal was then perfused with $1000 \mathrm{ml}$ of $4 \%$ paraformaldehyde in $0.1 \mathrm{M}$ phosphate buffer solution at a pH of 7.2 - 7.4. Both of these fluids were passed at a rate of $40 \mathrm{ml} \mathrm{min-1}$ at room temperature. As peroxidase activity is decreased by exposure to aldehydes it was imperative to rinse away all the fixation fluids. For this purpose $500 \mathrm{ml}$ of $10 \%$ sucrose in $0.1 \mathrm{M}$ phosphate buffer solution, followed by $500 \mathrm{ml}$ of a $20 \%$ sucrose in $0.1 \mathrm{M}$ phosphate buffer solution were perfused through the animal at a rate of $60 \mathrm{ml} \mathrm{min-1}$ at a temperature of $4^{\circ} \mathrm{C}$. All perfusion fluids were passed using a peristaltic perfusion pump (Harvard Apparatus).

Exposure to aldehydes has been shown to decrease the enzymatic activity of HRP (Courville and Saint-Cyr, 1978; Mesulam, 1982). Therefore, in our perfusions of HRP material aldehyde exposure never exceeded 30 minutes. A $4 \%$ paraformaldehyde solution was used for fixation, rather than a glutaraldehyde/paraformaldehyde mix as the former solution has been shown to produce a more consistent fixation throughout the brain when short fixative exposures are used as they are for WGA-HRP containing tissue (Mercier, 1989). Fixation with paraformaldehyde without glutaraldehyde has also been 
shown to result in less background artefact (Gibson et al., 1984). These procedures ensured a good, consistent fixation of tissue and minimal degradation of the HRP catalytic activity. Protocols for all solutions are given in the Appendix (page 221).

\section{Perfusion Of BDA Injected Rat}

After a survival time of 7 day the animal was lightly anaesthetised, and then killed by asphyxiation with $\mathrm{CO}_{2}$. The animal was prepared for perfusion as with the WGA-HRP animals. Blood was rinsed from the animal using $1000 \mathrm{ml}$ of a $0.9 \%$ saline solution containing 1000 units of heparin. Fixation was produced by passing $1000 \mathrm{ml}$ of a mixture of $4 \%$ paraformaldehyde with $0.05 \%$ glutaraldehyde in 0.1 phosphate buffer solution through the animal. All the solutions were passed at a rate of $40 \mathrm{ml} \mathrm{min}^{-1}$ through a peristaltic pump at room temperature. Following perfusion the brain was removed and stored in $20 \%$ sucrose in $0.1 \mathrm{M}$ phosphate buffer solution at $4^{\circ} \mathrm{C}$. Protocols for all solutions are given in the Appendix (page 226). 


\section{Histological Procedures}

\section{Tissue Sectioning}

Following the appropriate perfusion if the brain was to be cut coronally, the animal's head was replaced in the stereotaxic frame in a flat skull plane, incisor bar $3.3 \mathrm{~mm}$ below ear bar zero (Paxinos and Watson, 1982). The skull was opened and the brain was cut coronally towards the rostral end normal to the antero-postero axis. In this way the cut end of the brain became a flat surface on which it could be placed on the microtome stage so sections could be cut in a true coronal plane, normal to the flat skull plane. The brain was then removed from the skull and stored in $20 \%$ sucrose in $0.1 \mathrm{M}$ phosphate buffer solution. When the brain had sunk in the solution it was deemed to be impregnated with the sucrose solution and the tissue was considered cryoprotected and ready for cutting. Sinking the brain either took place overnight in a refrigerator at $4^{\circ} \mathrm{C}$ or the process could be accelerated by placing the brain in sucrose solution on an orbital shaker.

In some cases the cerebral cortex was removed and flattened. In this procedure the cerebral hemispheres were gently separated, and the corpus collosum cut with a scalpel, the cortex was then teased away from the underlying white matter. The cortex would then be placed in a petri dish containing $20 \%$ sucrose in $0.1 \mathrm{M}$ phosphate buffer solution, flattened, covered with a glass-microscope slide, weighted down with a $40 \mathrm{~g}$ weight and kept overnight at $4^{\circ} \mathrm{C}$. The flattened cortex was placed on the microtome stage, held in place with an embedding medium for frozen-tissue specimens (Tissue Tek, O.C.T. compound). The brain was then cut in a plane parallel to the flattened pial surface, 
starting from the surface. In these cases the midbrain and brainstem were cut as above in a coronal plane.

In all cases frozen sections were cut on a microtome (Leica polycut) at a thickness of 50 $\mu \mathrm{m}$. Some brains were cut throughout in a coronal plane, a marker was cut into the dorsal cortex and ventral brainstem in order identify the right left orientation of the sections. The brains were placed on a layer of ice on the microtome stage and held in place with water frozen around the base of the brain.

During cutting the brains were kept frozen by the application of solid carbon dioxide. Sections were removed from the microtome blade with a camel-hair brush. and placed into a plastic box with 25 bins containing a suitable storage medium. Three sections were placed in each bin, so each box held 75 sections. 
Neuronal Tracer Histology

Treatment Of HRP Containing Tissues

Sections cut from brains that contained WGA-HRP were stored in $0.1 \mathrm{M}$ phosphate buffer solution at $4^{\circ} \mathrm{C}$ prior to histological processing

$\underline{T M B}$

Every third section from the brain (i.e. every $150 \mu \mathrm{m}$ ) was taken for processing with the sensitive chromagen TMB. The TMB histology is a modification of the Mesulam (Mesulam, 1982) and Gibson et al. (1984) techniques.

Phosphate buffer solution was rinsed from the tissue with several washes in distilled water at $4^{\circ} \mathrm{C}$. During processing with $T M B$ care is taken that the reaction fluids and the reaction takes place at a temperature of $4^{\circ} \mathrm{C}$ buffered to a pH of around 3.3 with $0.025 \mathrm{M}$ acetate buffer. The tissue was reacted free floating in wells drilled into a perspex tray with plastic mesh covering the bottom of the wells. The system allowed for many sections to be processed simultaneously and the solutions to be changed quickly and easily.

After removing phosphate buffer solution from the tissue, the sections were placed in the reaction medium containing TMB for 40 minutes at $4^{\circ} \mathrm{C}$ to allow the tissue to become fully impregnated with the reaction solutions. The reaction was then initiated by adding $1 \%$ $\mathrm{H}_{2} \mathrm{O}_{2}$. The reaction with $T M B$ took place for 40 minutes at $4^{\circ} \mathrm{C}$ with agitation. Sodium nitro 
ferracyanate is used as a reagent to stabilise the HRP-TMB reaction product (Goodpasture, 1919). However, precipitation of sodium nitro ferracyanate crystals from the reaction solution can result in artefact in the tissue. To reduce the possibility of this artefact the reagent solutions were kept chilled, changed at 10 minute intervals and the concentration of sodium nitro ferracyanate in solution was kept relatively low $190 \mathrm{mg}$ $\left.1000 \mathrm{ml}^{-1}\right)$. These modifications produced TMB reacted HRP material with high sensitivity and specificity, with a low incidence of background artefact.

After the reaction the sections were rinsed with $0.025 \mathrm{M}$ acetate buffer and then mounted onto subbed slides from the same solution. The sections were air dried until ready for coverslipping. Care had to be taken during dehydration of material prior to coverslipping due to the solubility of the TMB reaction product in alcohol. Sections were minimally exposed to acetate-buffered alcohols, around 10 seconds in each alcohol of increasing concentration, cleared in xylene and coverslipped using DPX mounting medium. Using these procedures there were no problems with under dehydrated TMB reacted material. Detailed TMB protocols are given in the appendix (page 221).

\section{$\underline{D A B}$}

A set of sections at every $300 \mu \mathrm{m}$ were taken to correspond with the injection site in the pontine nuclei and processed with the chromagen DAB. Sections were processed free floating in the $\mathrm{DAB}$ reaction solution buffered to around a $\mathrm{pH}$ of 7.3 with $0.1 \mathrm{M}$ phosphate buffer solution in perspex bins for half an hour, rinsed thoroughly with, and mounted onto subbed slides from $0.1 \mathrm{M}$ phosphate buffer solution. After air drying the sections were dehydrated in increasing concentration alcohols, cleared in xylene and coverslipped 
using DPX mounting medium. The DAB reaction product is not soluble in alcohol so normal (around 3 minutes) exposure to solutions of alcohols of increasing strengths were used to ensure total dehydration. Detailed DAB protocols are given in the appendix (page 225).

\section{Biotinylated Dextran Amines}

After cutting, sections containing BDA were stored in $0.9 \%$ saline in 0.1 phosphate buffer solution. Prior to the reaction sections were rinsed in $0.9 \%$ saline in 0.1 phosphate buffer solution with $0.3 \%$ of the detergent triton added. Sections were reacted using a avidin-

biotinylated HRP (ABC, Vectastain) procedure slightly modified from the methods of Veenman and Honig (/d (Veenman et al., 1992) and Wouterlood and Jorritsma-Byham (1993). The sections were then rinsed in $0.1 \mathrm{M}$ phosphate buffer solution and the resultant HRP-avidin-biotin complex visualised by reaction with the chromogen DAB with hydrogen peroxide in $0.1 \mathrm{M}$ phosphate buffer solution. The sections were then mounted onto subbed slides from 0.1 phosphate buffer solution, air dried, dehydrated and coverslipped in a similar way as the DAB reacted HRP material. A detailed protocol will be given in the appendix (page 226).

Cell, Myelin And Enzyme Histology

Fibre Stain 
To identify the site of the lesion a set of sections was taken at an interval of either $150 \mu \mathrm{m}$ or $300 \mu \mathrm{m}$ throughout the level of the basis pedunculi and stained for myelin. I used a modification of the Heidenhain procedure (Heidenhain, 1892; Hutchins and Weber, 1983)) which allowed us to stain free floating sections. The method produced fibre stains of a less blotchy, and of a more consistent appearance than those produced with previous Heidenhain methods that stained sections already mounted on glass slides. Blotchy staining on sections that have been previously mounted onto glass slides can be caused by trapped hematoxylin between the tissue and the slide, in the method used the sections were stained free floating in bins, thus eliminating this particular problem. Inconsistent staining can be caused by uneven hydration of tissue before staining. The rapid hydration of the section utilised ensured consistent hydration and therefore a more even stain. A detailed protocol for fibre staining is given in the appendix.

\section{Nissl Stain}

A series of sections was always taken through the entire brain at intervals of either $150 \mu \mathrm{m}$ or $300 \mu \mathrm{m}$, these sections were reacted with TMB (as above). These sections were then counterstained with the Nissl stain, cresyl violet. These nuclear stained sections allowed us to identify accurately the location of the injection site in the pons, the laminar location of retrogradely labelled cells in the cortex, and were helpful in reconstructing the lesion sites. In some cases in which the TMB reaction product in the area of the injection was so dense as to obscure the underlying structures a corresponding set of sections that had not been reacted with a HRP chromagen were taken through the brain stem and stained with cresyl violet to aid reconstruction of the injection site in the pontine nuclei. 


\section{Cytochrome Oxidase}

To identify the position of the barrels in both coronal sections and sections of flattened cortex tissue was incubated in a reaction medium containing cytochrome c, catalase, $\mathrm{DAB}$, and hydrogen peroxide in $0.1 \mathrm{M}$ phosphate buffer solution. In this way the barrels were visualised as areas containing relatively high concentrations of cytochrome oxidase. The reaction took place over a period of hours at room temperature, or overnight at $4^{\circ} \mathrm{C}$, either procedure produced similar high quality histological sections. A detailed protocol for the cytochrome oxidase reaction is included in the appendix. 


\section{Analysis Of Data}

\section{Behavioural Data}

The performance of an animal at each gap distance was measured as a percentage of successful crosses against attempts to cross. Therefore, if the animal crossed every time he left the box at a given distance, his success at that distance was $100 \%$. Information of this type was calculated for the largest stable distance before and after whisker shave, and in some cases as the whiskers re-grew. The information was then plotted as a percentage success at the maximum pre-whisker cut distance against trial day. In this way I could observe the success of an animal at a given gap size of over time.

Another graphical method of displaying the success of the animal was also used. Using the same method as Hutson and Masterton (Hutson and Masterton, 1986) I simply displayed the percentage success of the animal pre and post-whisker cut plotted aganst a given distance.

Notes from observing the behaviour of the animal during the entire training and testing process were continually taken in an attempt to observe any subtle behavioural changes, or changes in strategy for completing the task.

\section{Anatomical Data}




\section{Injection Sites}

There is no universally agreed criterion to determine the area of effective uptake of WGAHRP at an injection site (Jones, 1975). Sections containing the injection site in the pontine nuclei were reacted with two chromagens, $D A B$ and the more sensitive TMB. Inspection of the injection site in sections reacted with TMB showed an inner dense area of staining around the injection site surrounded by a paler "halo". Comparisons of these sections with corresponding sections reacted with $D A B$ indicated that the dark brown precipitate produced in the DAB reaction matched the extent of the inner dense TMB stain.

There is evidence for and against uptake from the area encompassed by the TMB "halo" (Bernard, 1987; Dursteler et al., 1977). Sections including the injection site were traced using a stereomicroscope with a camera lucida drawing tube attached (Wild W8). In all cases the limits of the spread of WGA-HRP at the injection site were drawn from sections reacted with DAB (Jones and Hartman, 1978). A similar procedure was used in the cases were the injection site was in the cortex regardless of the whether the tracer was WGAHRP or BDA.

Drawings were scanned as templates into the vector based illustration package (Adobe Illustrator v.7) from a flatbed scanner (Sharp JX-330P). Using these templates it was possible to produce accurate representations of the injection site throughout the pontine nuclei. The drawings were then presented as serial sections through the area of interest. An accompanying photomicrograph of one of the drawn sections allowed for comparison with the graphical representations. 
Injections sites within the cortex were drawn over a reconstruction of the barrel fields taken from serial sections reacted for cytochrome oxidase. Serial sections were traced onto tracing paper and aligned using the barrels, blood vessels, the section outline and markers placed in the section with a fine, hollow plastic needle before they were flattened. Once aligned the barrels were drawn on a single composite of the entire barrel field which then had the injection site superimposed on it. The resulting drawing was scanned, and reproduced on the computer for presentation.

\section{Lesion Sites}

Using sections stained for myelin and with cresyl violet lesions of the cerebral peduncle were located and traced using the same procedure used for the injection site. The drawings were then reproduced on the computer and presented as serial sections through the lesion. A parallel photomicrograph of one of the sections allowed comparison between photographic and graphical representations of the lesion.

\section{Cellular And Terminal Label}

Coronal sections containing cells labelled with retrogradely transported WGA-HRP were viewed and photographed at low and medium power using either a Nikon E800M microscope with a Spot II digital camera attached (Diagnostic instruments), or on an Olympus $\mathrm{BH}-2$ microscope with a conventional $35 \mathrm{~mm}$ camera attached /Olympus C35AD-4 camera with Olympus exposure control unit). Corresponding sections which had 
been reacted for cytochrome oxidase were then either drawn and reproduced as whole sections on the computer, or photographed at the same power in order to demonstrate the regions within which the barrels were found corresponding to the retrograde label. The sections were presented as serial sections through the brain.

For flattened cortices that contained retrogradely labelled cells a composite drawing of the barrel field was constructed as has been described. Onto this a composite photograph of the sections containing labelled cells placed in alignment with markers. The finished composite diagram indicated the barrels and the areas in which labelled cells were present.

Neuronal terminals in the pontine nuclei following injections of HRP-WGA into the barrel field were drawn with a camera lucida drawing tube, transferred onto computer and reconstructed in a manner similar to that utilised for the reconstruction of pontine nuclei injection sites described above. 
RESULTS 


\section{Case 1}

The lesion of the cerebral peduncle is shown in Figure 11. The lesion was placed centrally in the peduncle's rostro-caudal axis, and slightly above the dorso-ventral middle. In this position it would have cut many of the efferent fibres that are labelled in the peduncle after injections of anterograde tracer into the vibrissal barrel field (Glickstein et al., 1992).

The damage was largely restricted to the medial side of the cerebral peduncle, though in many sections the damage is almost complete leaving only a small number of peduncle fibres on the very lateral edge intact (Figure 11, section 77-81).

The animal was initially trained to jump the gap at a stable distance; first in the light and then dark, it took about four weeks achieve stable performance under both conditions. Once stable the animal had all the whiskers on the on the right side of the face cut to their bases, and immediately tested to see whether this manipulation had any effect. After testing the animals whiskers were allowed to re-grow, it took the whiskers just over two months to re-gain their normal length. The animal then underwent surgery to interrupt the central fibres of the right cerebral peduncle. Re-training commenced four days after surgery. It took four, five day training blocks for the rat to become stable again at pre-operative distances. Once stable the vibrissae on the right side of the face were again cut to there bases, and the animal tested. As the whiskers re-grew the animal was kept in training, this comprised of 42 training days spread over approximately three months as the whiskers grew back to their normal size. At this point the whiskers on the left side of the face were cut to their bases and the animal tested to see if it the rats behaviour. This cut and tests marked the end of this rats behavioural 
testing. From beginning to end the training and testing took just over seven months, which is typical for extended behavioural testing.

Figure 12 and 13 show the sequence of behavioural results from these manipulations. Starting with Figure 12 (i) which shows that with both peduncles and both sets of whiskers intact the animal consistently and stably crossed a gap of $15 \mathrm{~cm}$ in the dark. At this distance the animal used only its whiskers to gauge the distance to the far edge of the gap. At smaller distances the animal used a combination of vibrissae, nose and paw to gauge the distance. At gaps over $15 \mathrm{~cm}$ in the dark the animal could not touch the far side with any part of its body including its vibrissae, at these distances the animal never crossed.

The whiskers on the right side of the rat's face were cut to their bases. Figure 12 (ii) shows that leaving only the left whiskers with both peduncles intact has no effect on the animal's levels of performance. The rat used the left whisker successfully to gauge the distance to cross.

Whilst the animal's whisker were re-growing it underwent surgery to cut the appropriate fibres in the right cerebral peduncle. After the animal had recovered from surgery and the whiskers had regained their original length it was re-tested. Figure 12 (iii) shows that with a lesion in the right peduncle and both sets of whiskers intact the animal performed at the same levels as it had before any surgery.

The whiskers were again allowed to re-grow, and the animal was tested and shown to still be able to cross a gap of $15 \mathrm{~cm}$ in the dark to stable criterion, at which point the whiskers on the right side of the face were cut to their bases. Figure 12 (iv) shows that 
cutting the right vibrissae produced a marked change in the animal's performance. After the right whiskers were cut the animal now refused to cross a gap of $15 \mathrm{~cm}$ in the dark, even though the remaining whiskers on the left side of the face could be seen to make contact with the far edge of the gap. The animal stabilised at crossing a gap of $13 \mathrm{~cm}$ in the dark. At this distance the animal would invariably make contact with his nose or paw before crossing. The rat was tested after its whiskers had re-grown, after which performance returned to normal as the whiskers re-grew.

Once at their original length the animal tested to show it would still stably cross at a gap of $15 \mathrm{~cm}$ in the dark. The whiskers on the left side of the face were then cut to their bases. Figure $12(\mathrm{v})$ shows that with only the vibrissae of the right side of the face intact the animal would still cross a gap of $15 \mathrm{~cm}$ in the dark. At this distance the animal would always be seen to make contact with the far side of the gap with its remaining right whiskers before crossing.

During all the testing in the dark the rat was tested in the light and would consistently crossed a gap of $34 \mathrm{~cm}$ regardless of the condition of the right peduncle or the vibrissae.

We could not verify the position of the peduncle lesion by considering the distribution of retrogradely labelled cells in the cortex as the animal died during surgery to fill the pontine nuclei with WGA-HRP. 
Figure 11.

Case I.

Peduncle Lesion.

76

77

78

79

80

81

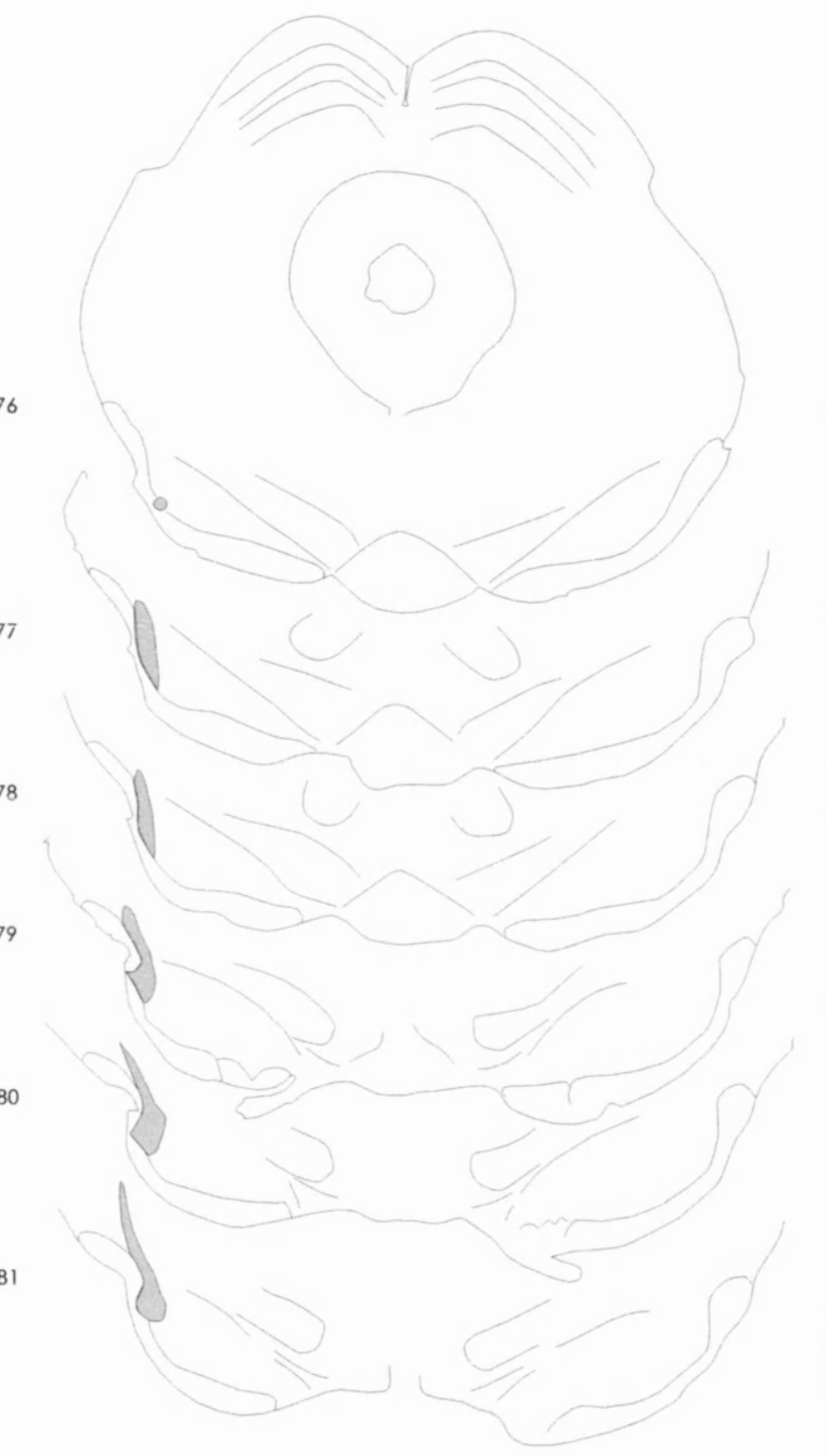

82

83

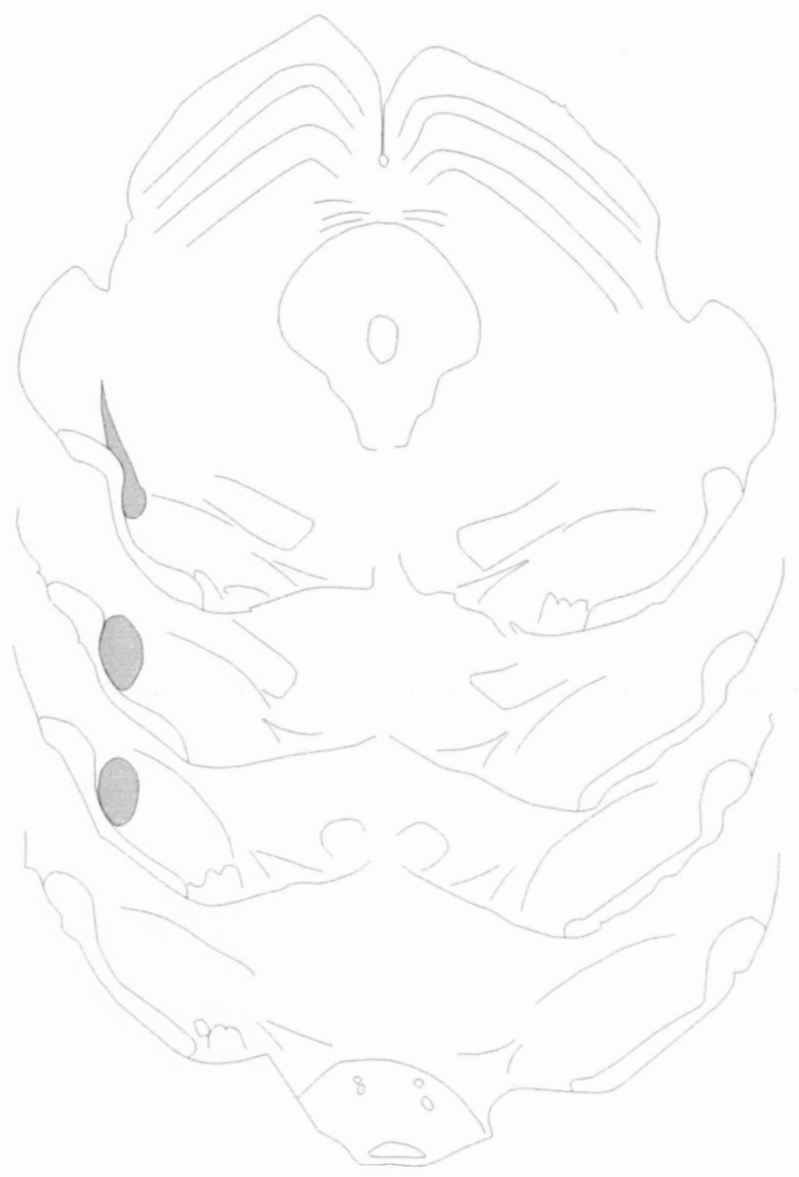

$1 \mathrm{~mm}$

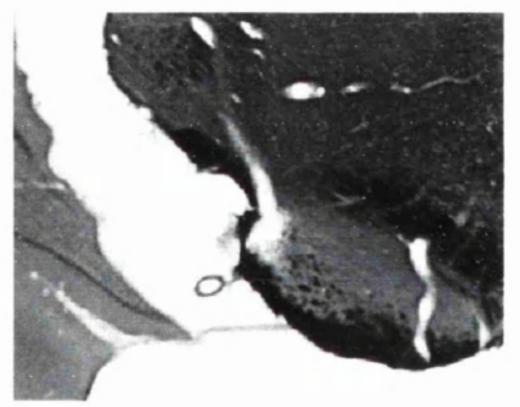

Above:

Lesion in the cerebral peduncle of Case I.

Sections are 150 microns apart.

Left:

Photomicrograph of lesion. Section 80. 
Figure 12. Case I.

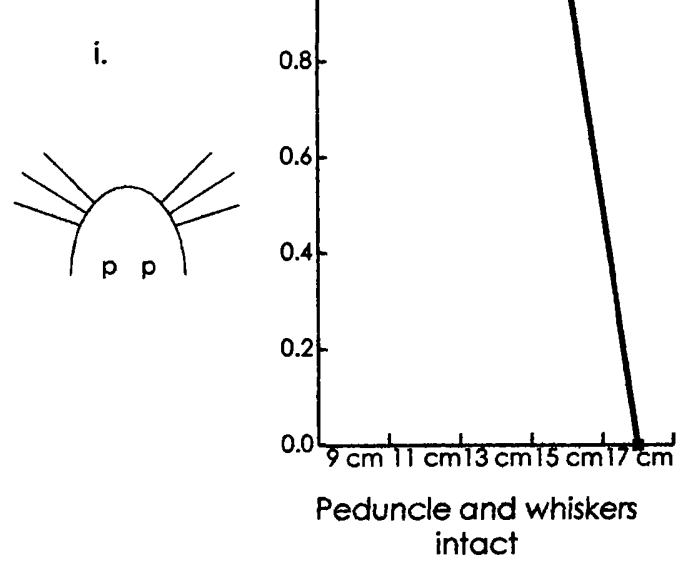

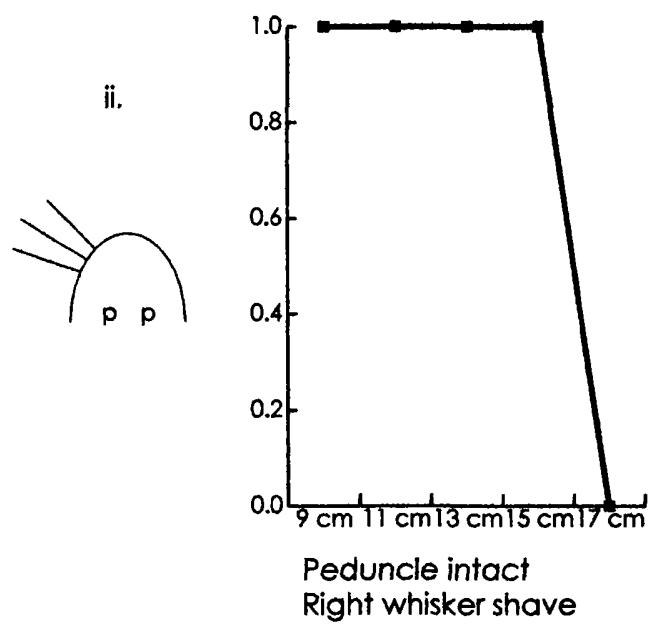

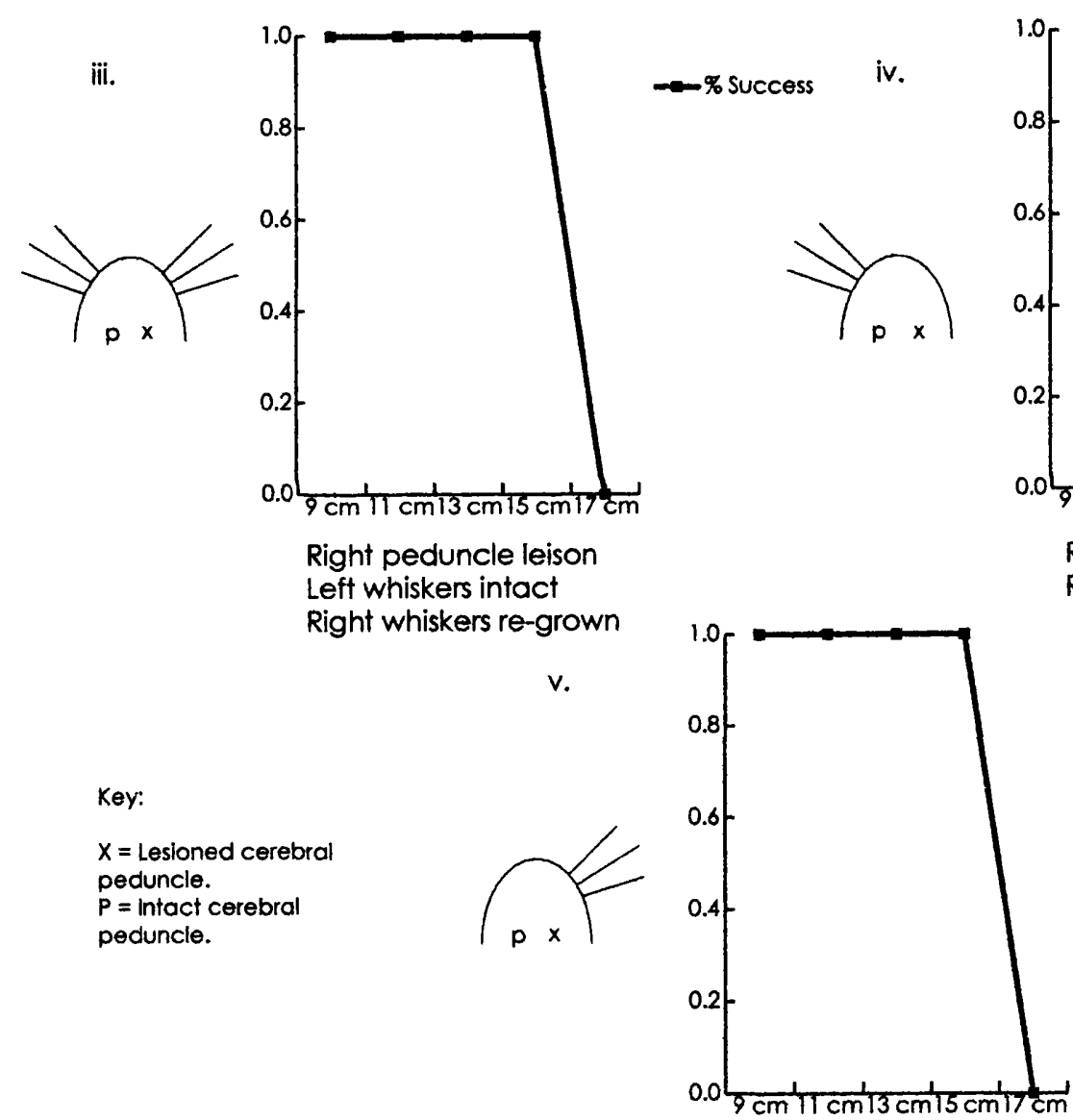

Right peduncle lesion; right whiskers regrown left whisker shave

Graphs showing the effect of different combinations of whisker shaves and peduncle lesions on the maximum distance the animal would cross in the dark. 
Figure
casel.

$$
\% \text { success at } 15 \mathrm{~cm}
$$

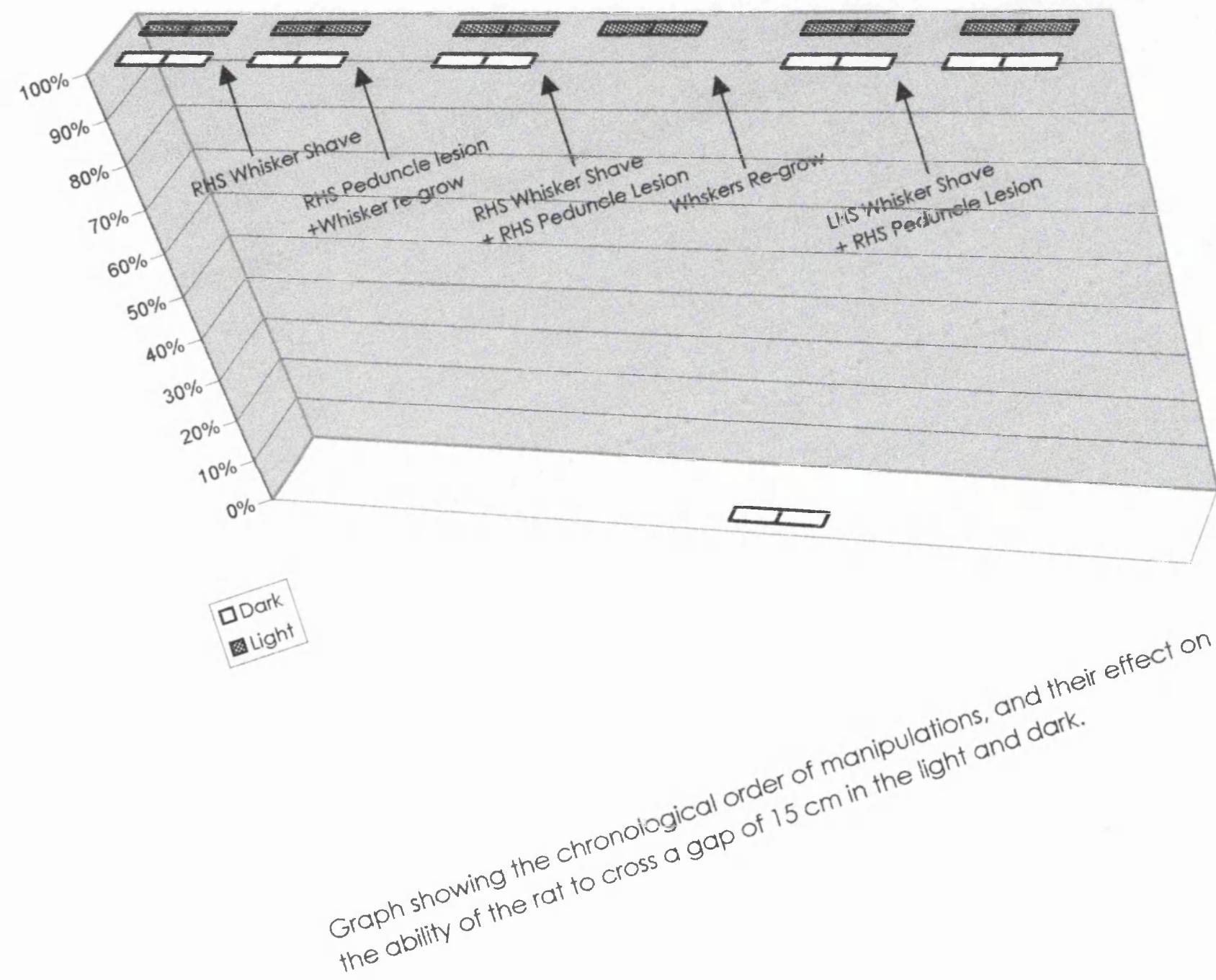




\section{Case II}

The cerebral peduncle lesion of this case is shown in Figure 14. The lesion is very large and centred just above the dorso-ventral middle of the peduncle well placed to cut efferent fibres from the vibrissal-barrel field. The lesion would have also destroyed fibres from posterior areas of cortex i.e. visual areas of cortex that run in the dorso-lateral region of the peduncle (Glickstein et al., 1992). The lesion also encroached into the dorso-lateral part of the substantia nigra pars reticularis.

Behaviourally the animal was tested first as normal, then with intact whiskers following the lesion in the right peduncle. Finally, the rat was tested with the lesion in the right peduncle and the right vibrissae shaved.

Figure 15 shows the effect of the different manipulations on the maximum distance that the animal would cross in the dark. Figure 16 shows the chronological order of the manipulations, and their effect on the animal's success at crossing a gap of $16 \mathrm{~cm}$ in the light and dark.

Figure 15 (i) and 16 show that the rat was originally trained to consistently cross a $16 \mathrm{~cm}$ gap in the dark. At this distance in the dark the animal could be seen to make contact with the far edge of the gap with only its whiskers, the rat invariably made contact in this manner before crossing. At smaller gaps the animal would use vibrissae, nose and paw to judge the distance to the far edge.

Once performance was stable the animal underwent surgery to cut the fibres of the cerebral peduncle that carry efferent fibres from the vibrissal barrel cortex. After 
recovery from surgery the animal was re-tested. Figure 15 (ii) shows that the unilateral peduncle lesion had no effect on the maximum distance the animal would cross in the dark, nor the consistency of its performance (Figure 16).

The whiskers on the right side of the face were then cut to their bases. Cutting the vibrissae on the right produced a marked change in the animal performance and behaviour. Figure 15 (iii) shows that the maximum distance that the rat would cross in the dark was reduced by $2 \mathrm{~cm}$ to $14 \mathrm{~cm}$. The rat never crossed at a gap of $16 \mathrm{~cm}$, the previous maximum distance in the dark (Figure 16), although the remaining vibrissae on the left side of the face could be seen to make contact with the far edge of the gap. The rat would only cross a gap which it could reach across with its nose or paw.

Throughout training and testing the rat was given a similar task in which it had to use vision to gauge its jump. Throughout training and testing the animal consistently crossed a gap of $24 \mathrm{~cm}$ in the light using vision to gauge its jump.

The interruption of cortico-pontine fibres was verified by the position of terminal label in the pontine nuclei following injections of similar amounts of $10000 \mathrm{M} . \mathrm{W}$. BDA into each vibrissal barrel field. Figure 17 shows that the injection into the right PMBSF was centred over the E3 barrel. Figure 18 shows that the left cortex injection was centred slightly more medially, over the D3 barrel. Though the injections were of a similar volume the spread of the injection in the right hemisphere (Figure 17) is less than that seen in the left hemisphere (Figure 18).

Fibres labelled after injection of BDA into the PMBSF are seen in an area of the peduncle contralateral to the lesion that mirrors the position of the fibres destroyed by the lesion. 
Terminal label within the pontine nuclei contralateral to the cerebral peduncle lesion was distributed in a manner consistent with that of earlier studies describing pontine label following injections of WGA-HRP into the facial sensory area of the primary somatosensory cortex (Mihailoff et al., 1985)

Distribution of terminal label on the pontine nuclei are shown in Figure 19. Label in the side of the pontine nuclei corresponding to the side of the lesioned peduncle was much sparser. In some areas the label was only seen contralateral to the lesion (Figure 19, sections $58 \& 62)$. In others the label was seen in areas similar to those on the contralateral side though the labelled area was smaller and less dense (Figure 19, sections $56,57,59 \& 60$ ). In only one section was label seen only on the side ipsilateral to the peduncle lesion (Figure 19, section 61).

The injection site in the left PMBSF is larger than the injection in the right PMBSF, it would be possible to argue that this is the cause of the obvious bias of label toward the left side of the pontine nuclei. However obviously labelled fibres are seen in symmetrical positions in both peduncles, and this label is dramatically decreased on in the right peduncle below the level of the lesion. I would suggest that the extent of preferential bias of label in the pontine nuclei toward the left side is the result of the destruction of many of the descending fibres from the PMBSF that would have terminated in the pontine nuclei. 
Figure 14.

Case ll.

Peduncle lesion.
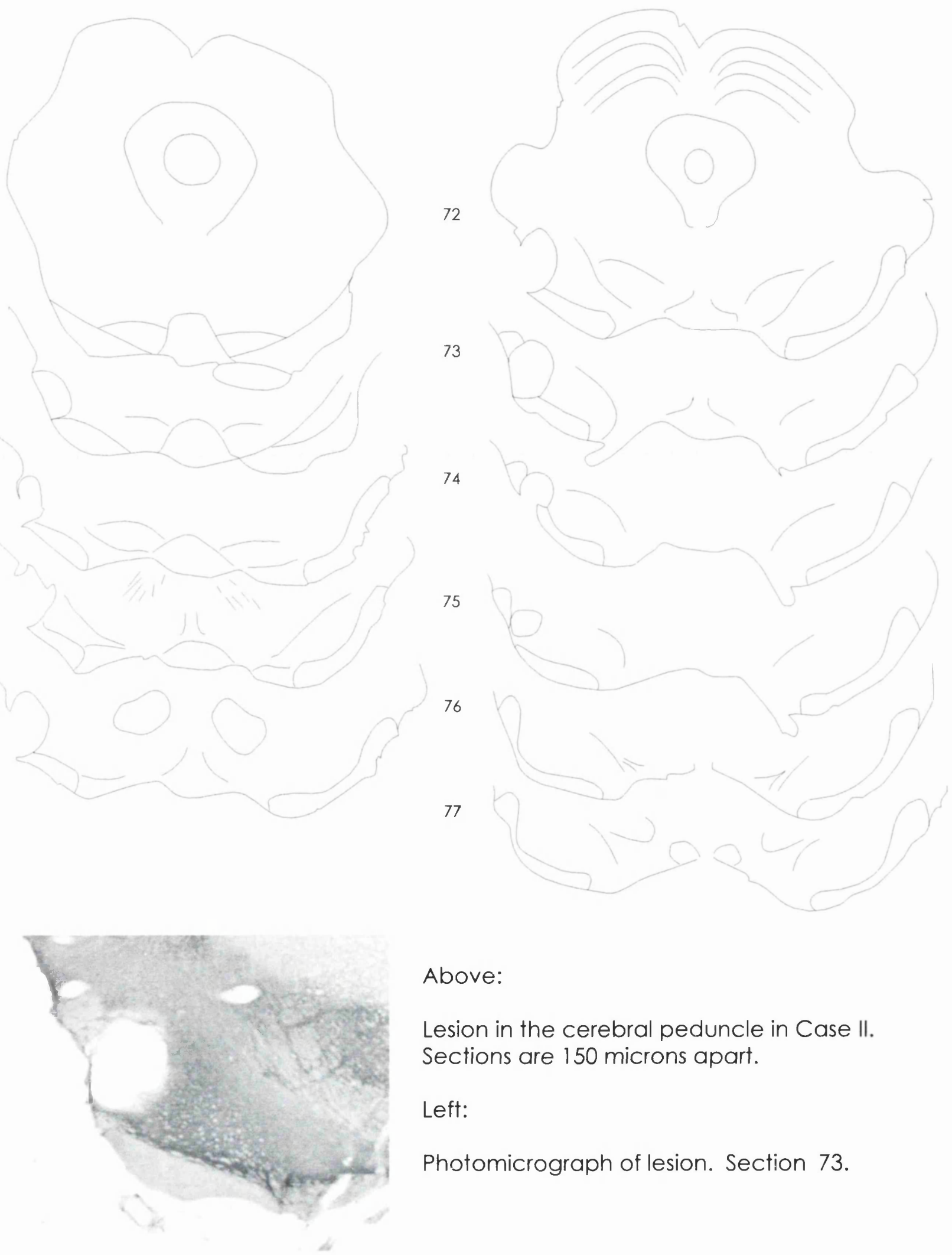

Above:

Lesion in the cerebral peduncle in Case II. Sections are 150 microns apart.

Left:

Photomicrograph of lesion. Section 73. 
Figure 15.

Case II.
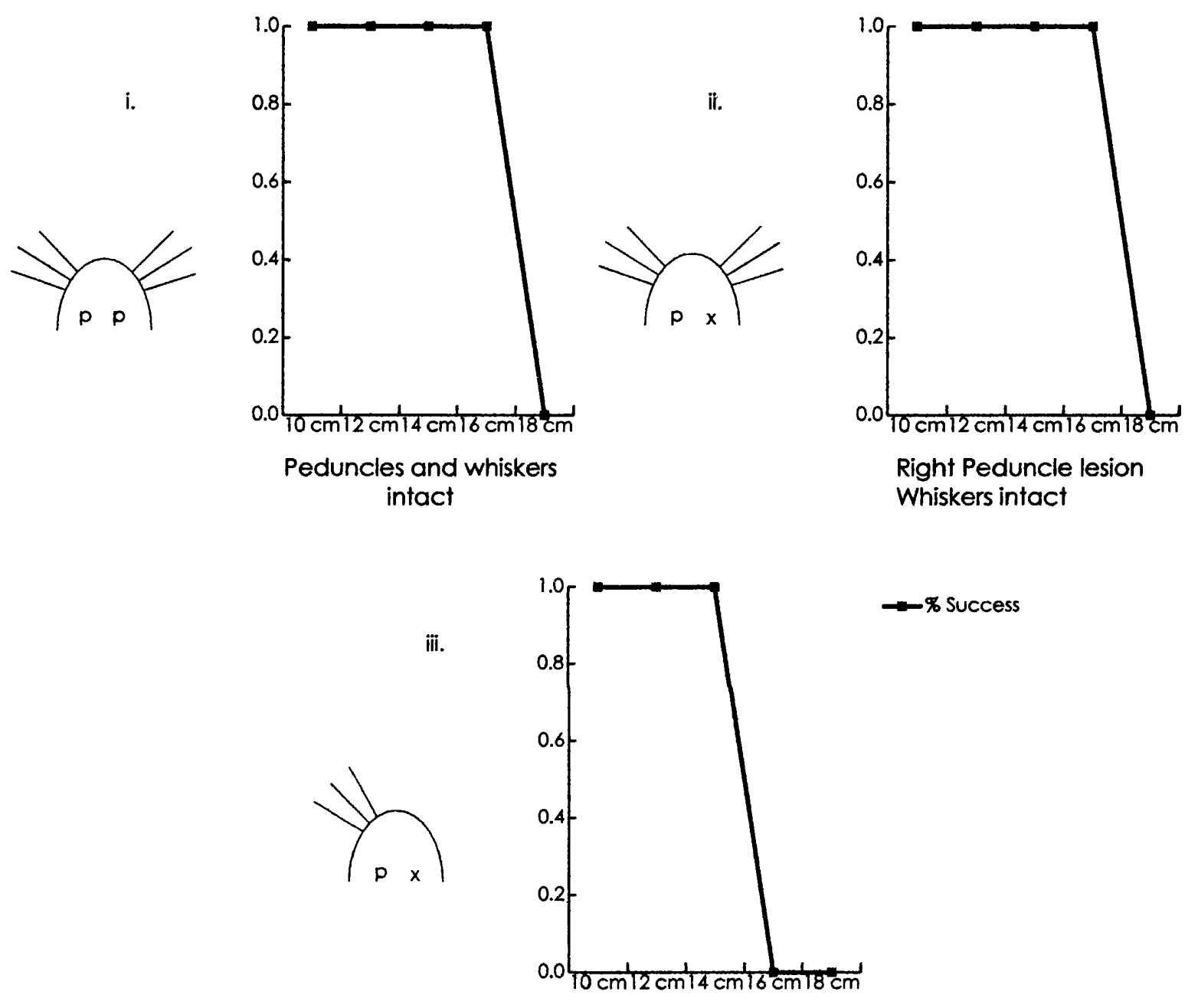

Right peduncle lesion Right whisker shave

Graphs showing the probability of the rat crossing at differing gap distances in its normal state (i), with a lesion in the right peduncle and both sets of whiskers (ii), and with a lesion in the right peduncle and only the left whiskers (iii). 


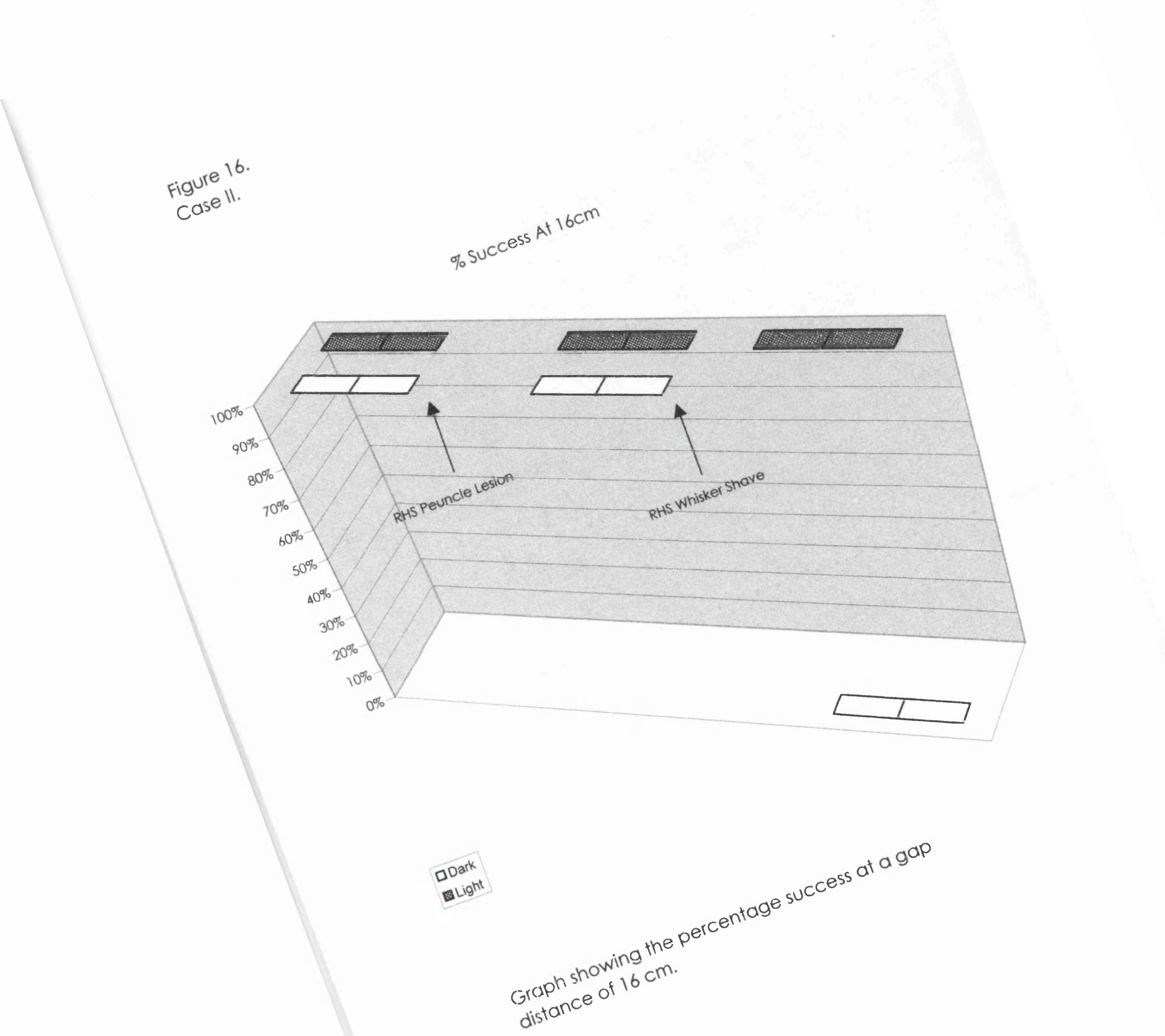


Figure 17.

Case ll.

B.D.A. Injection Site Right.

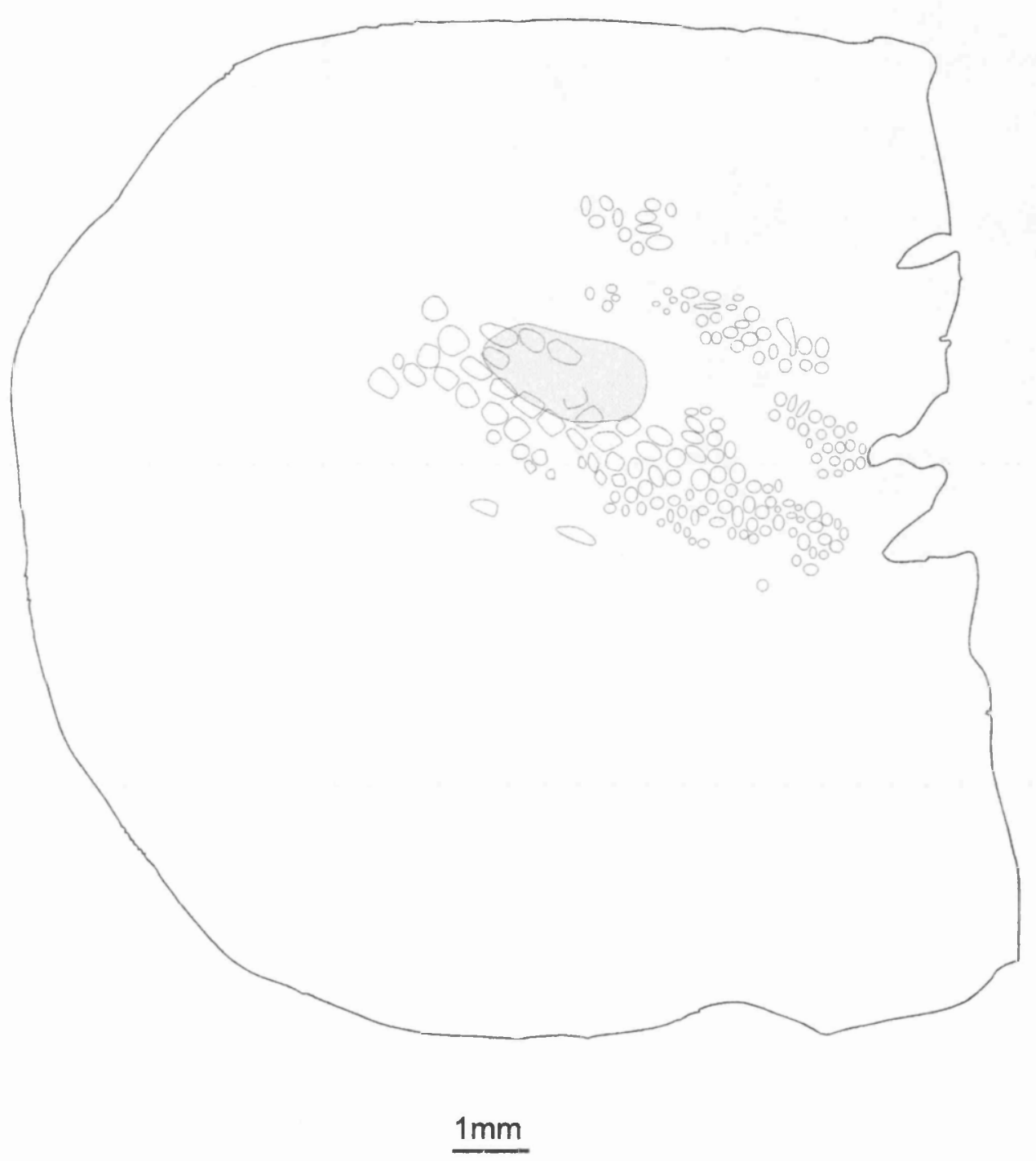

Flattened cortex of the right hemisphere. The shaded area represents the extent of the B.D.A. injection site. 
Figure 18.

Case 11.

B.D.A. Injection Site Left.

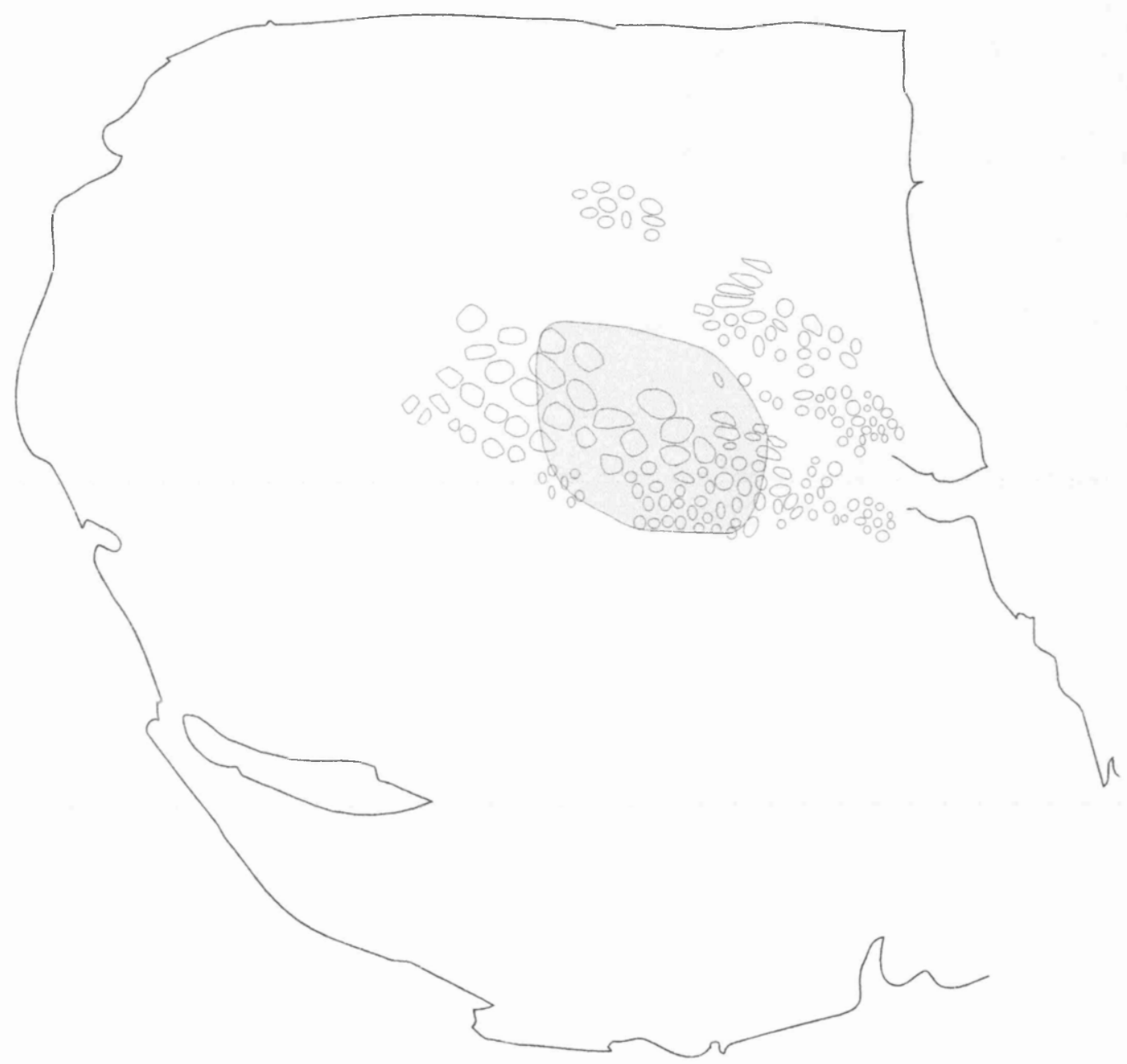

Flattened cortex of the left hemisphere. The shaded area represents the extent of the B.D.A. injection site. 
Figure 19.

Case II.

Pontine Nuclei Label.

52

53

54

55

56

57

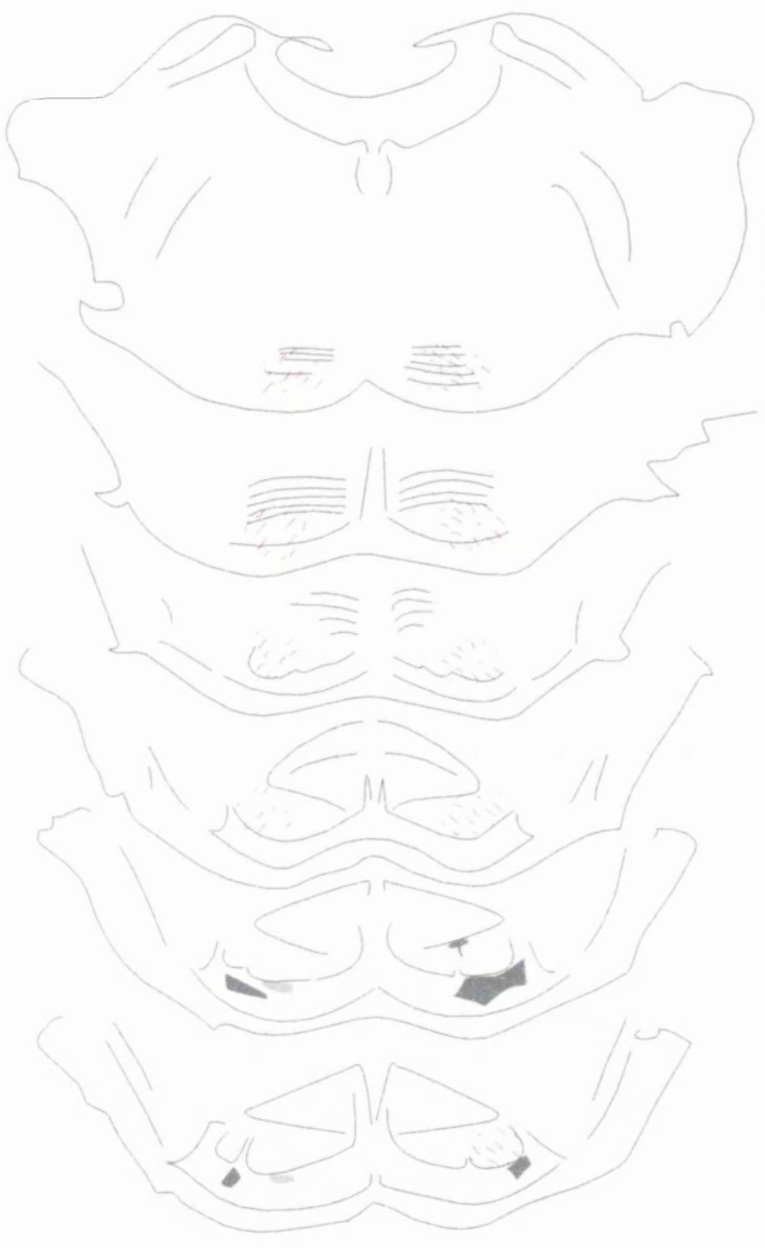

58

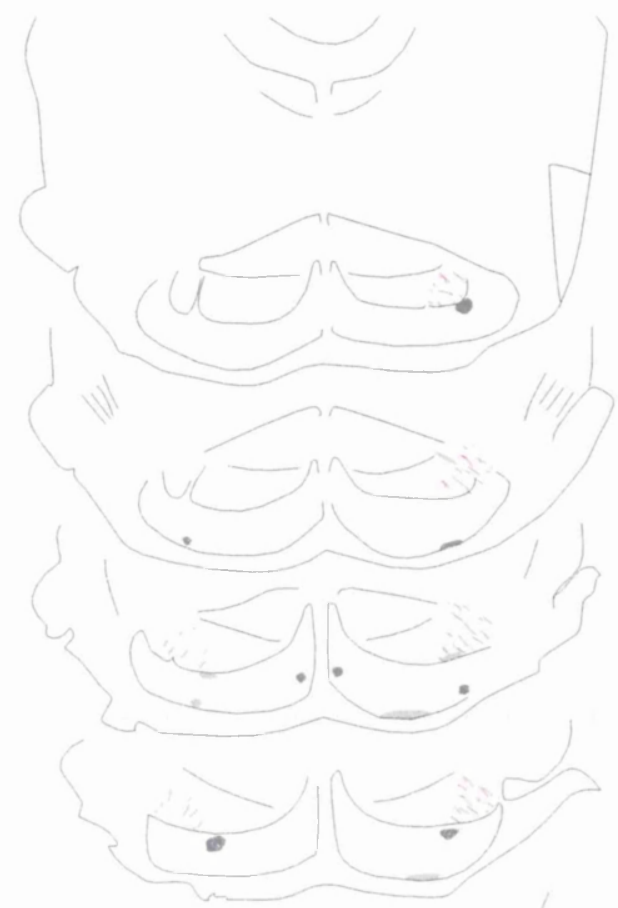

62

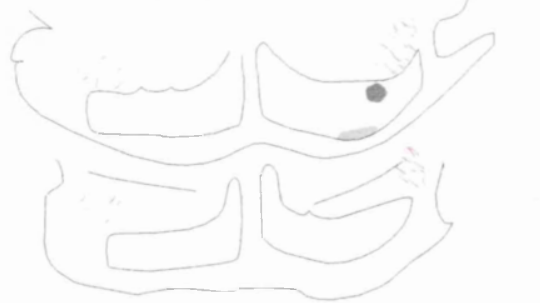

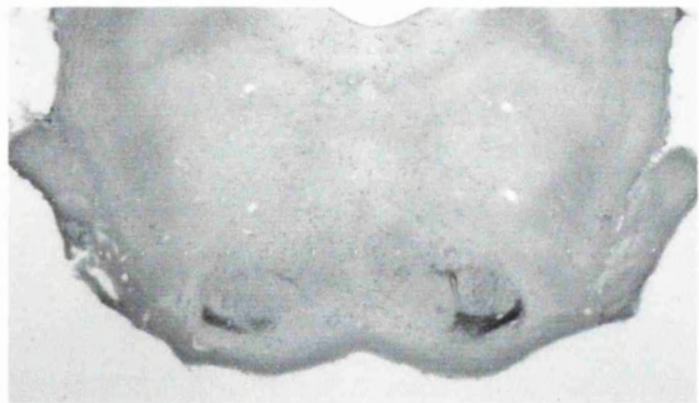

Above:

Terminal label in the pontine nuclei after bilateral injections of B.D.A. into the P.M.B.S.F. (Figures 17 \& 18). Red hatching represents labelled fibres. Grey fill represents terminal label, the density of the grey indicates the density of the terminal labelling.

Left: Photomicrograph of section 56. 


\section{Case III}

The histological reconstruction of the lesion in the rat Case III is shown in Figure 20. The lesion is centrally placed in the rostro-caudal aspect of the peduncle at the level of the rostral end of the interpeduncular nucleus. The lesion would have interrupted many of the cortico-pontine fibres that arise in the PMBSF of the primary somatosensory cortex where they run just dorsal to the middle of the peduncle in its dorso-ventral aspect (Glickstein et al., 1992). The lesion also encroached somewhat on the dorso-lateral part of the substantia nigra pars reticularis.

Behaviourally the animal was first tested as a normal with both peduncles intact and a full complement of whiskers. Then the animal was re-tested with a lesion in the right cerebral peduncle. The rat was finally tested with the lesion in the right cerebral peduncle and only the whiskers on the left side of the face.

Figure 21 (i) and 22 show the rat would consistently cross a gap of $17 \mathrm{~cm}$ in the dark. At this distance the rat would always make contact with the far side of the gap with only its whiskers before crossing. At gaps smaller than $17 \mathrm{~cm}$ the animal would make contact first with paw, nose or vibrissae before crossing.

When performance was stable, surgery was performed on the animal to interrupt fibres in the central part of the right cerebral peduncle. After recovery from surgery the animal was re-tested. Figure 21 (ii) and 22 shows that the lesion itself did not affect the maximum distance of $17 \mathrm{~cm}$ that the animal would cross in the dark. As before, the animal used only its whiskers to gauge the gap at $17 \mathrm{~cm}$, but a combination of nose, paw and vibrissae at lesser distances. 
All the major whiskers on the right side of the face were then cut to their bases. Figure 21 (iii) shows that with only the left whiskers to gauge the gap the rat refused to cross its previous maximum of $17 \mathrm{~cm}$ in the dark. Although the remaining left whiskers could be seen contacting the far edge the animal never crossed a gap of $17 \mathrm{~cm}$ in the dark (Figure 22). The animal stabilised at crossing a maximum gap of $15 \mathrm{~cm}$, at this distance the animal would make contact with the far edge of the gap with its nose and paw before crossing.

The animal was also trained and tested in the light before and after each procedure. Under these conditions the rat consistently crossed gaps of $24 / 26 \mathrm{~cm}$ regardless of the condition of the whiskers or peduncles.

The origin of the cut fibres was confirmed by the distribution of retrogradely labelled cells in the cortex after the pontine nuclei had been filled with WGA-HRP. The extent of the injection site in the pontine nuclei is shown in Figure 22. The fill was total in the caudal $60 \%$ of the pontine nuclei (Figure 23, section $40-46$ ). In the very rostral part of the pontine nuclei and at the level of the transverse fibres of the pons the injection site was only contralateral to the lesion (Figure 22, section $50-52$ ). Between these sites the pons was fully labelled on the side contralateral to the lesion.

Ipsilateral to the lesion the injection site encompassed the central and lateral pons, areas which at this level receive efferent fibres from the vibrissal barrel field. The small medial area that receives input from the PMBSF at this level would have been partially labelled ipsilateral to the lesioned peduncle (Mihailoff et al., 1985). Label extends into the pontine reticular nuclei, the superior olive and the trapezoid body. 
Retrograde filling of the projection cells of layer $\mathrm{Vb}$ throughout the cortex would have been expected following such an extensive pontine nuclei fill with WGA-HRP (Legg et al., 1989). The distribution of HRP containing cells revealed after TMB histological processing is shown in Figure 23. In the left cortical hemisphere, contralateral to the cut peduncle, HRP labeled cells are seen throughout the whole of layer Vb. Ipsilateral to the lesioned peduncle very few cells were seen in layer $\mathrm{Vb}$ in the region of the barrel field. Areas medial to the barrel field contained the expected complement of labeled cells. These areas were identified as sensory hind and forelimb areas and frontal cortex.

The anatomical position of the lesion in the peduncle and the distribution of retrogradely labeled cells in layer $\mathrm{Vb}$ indicate that the lesion had interrupted most of the efferent fibres linking the PMBSF with the pontine nuclei. 
Figure 20.

Case III.

Cerebral Peduncle Lesion.

58

59

60

61

62

62
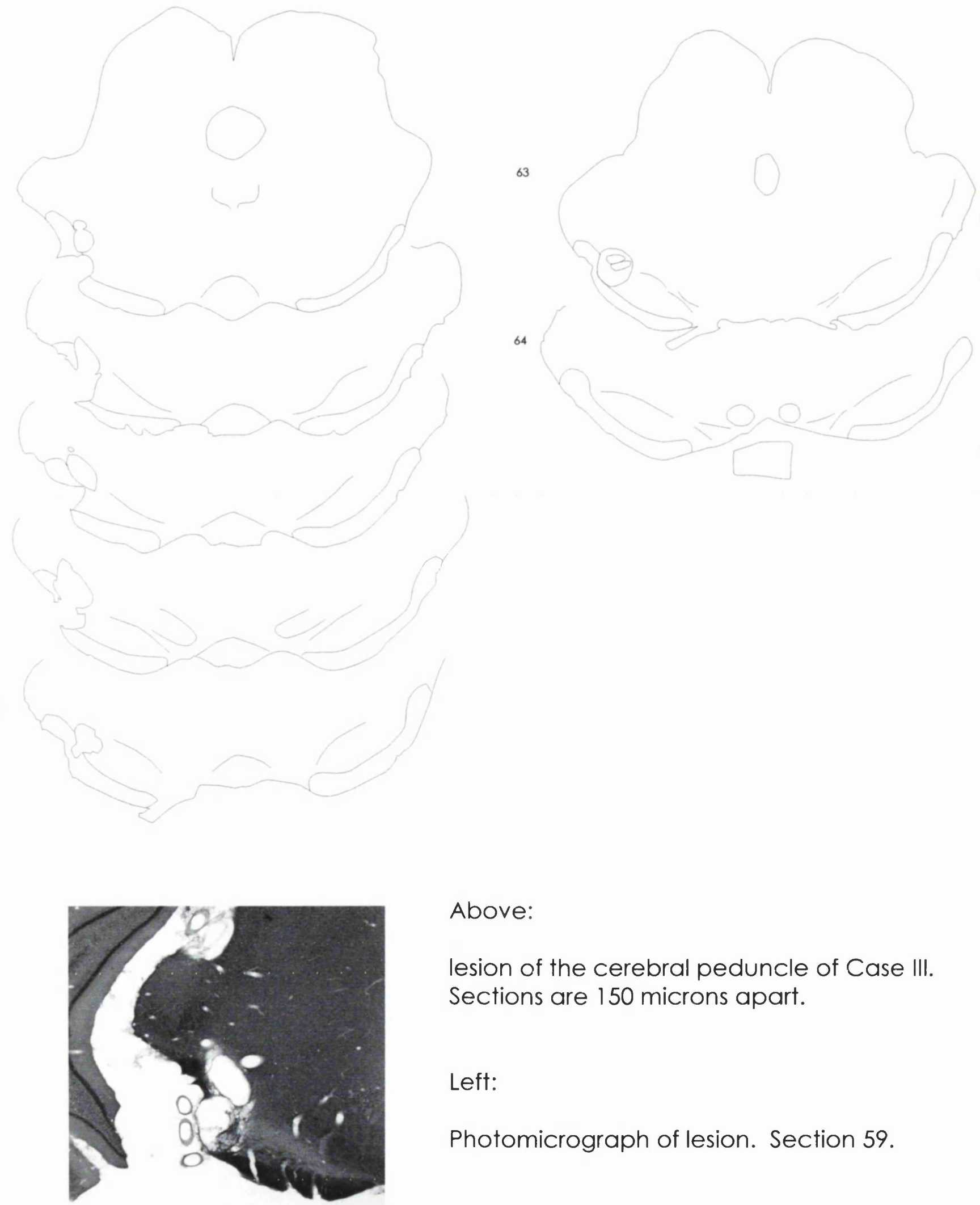

Above:

lesion of the cerebral peduncle of Case III. Sections are 150 microns apart.

Left:

Photomicrograph of lesion. Section 59. 
Figure 21.

Case III.
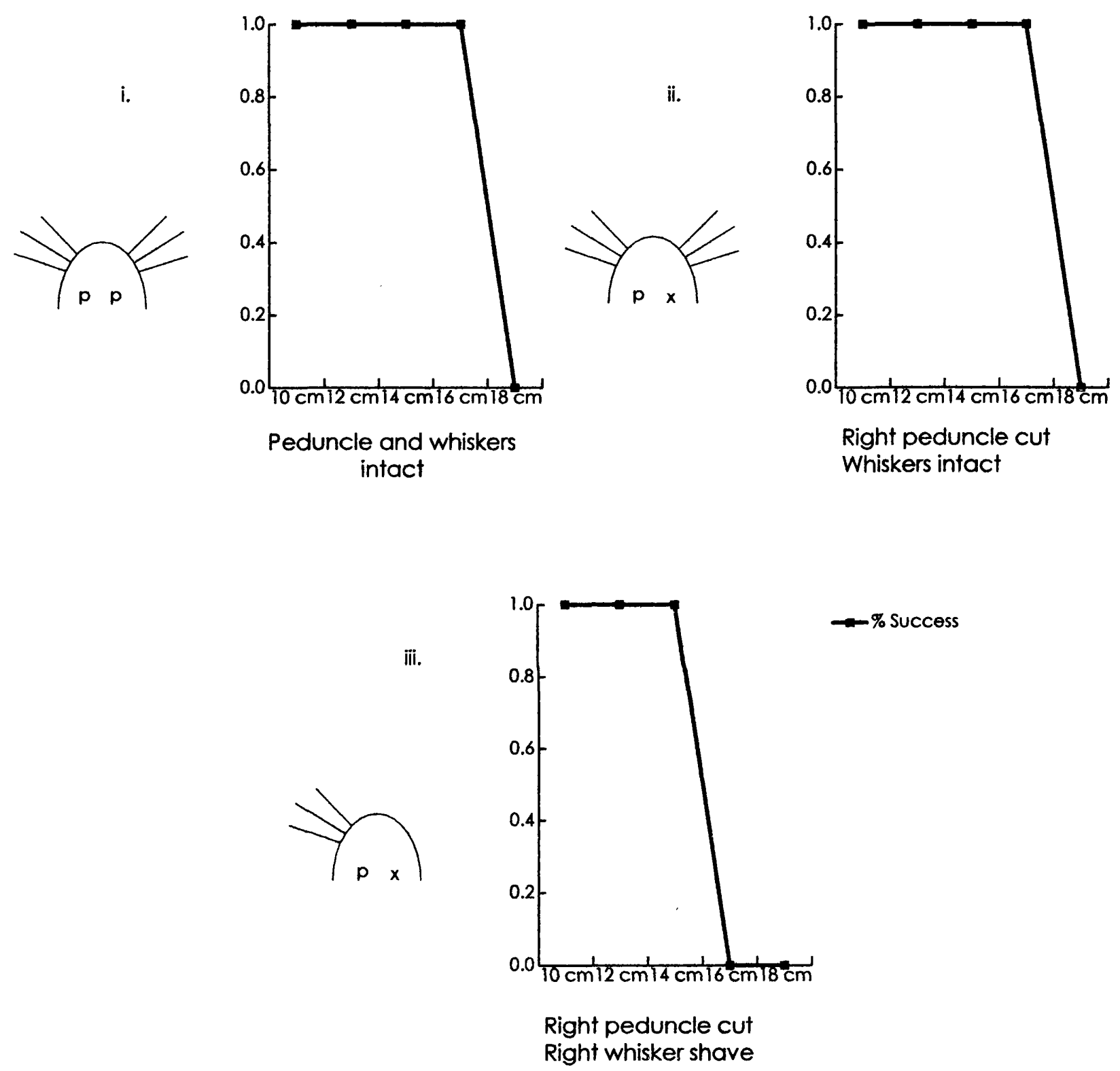

Graphs showing the probability of the rat crossing at differing gap distances in its normal state (i), with a lesion in the right peduncle and both sets of whiskers (ii), and with a lesion in the right peduncle and only the left whiskers (iii). 
Figure 22.

case III.

$\%$ Success At $17 \mathrm{~cm}$

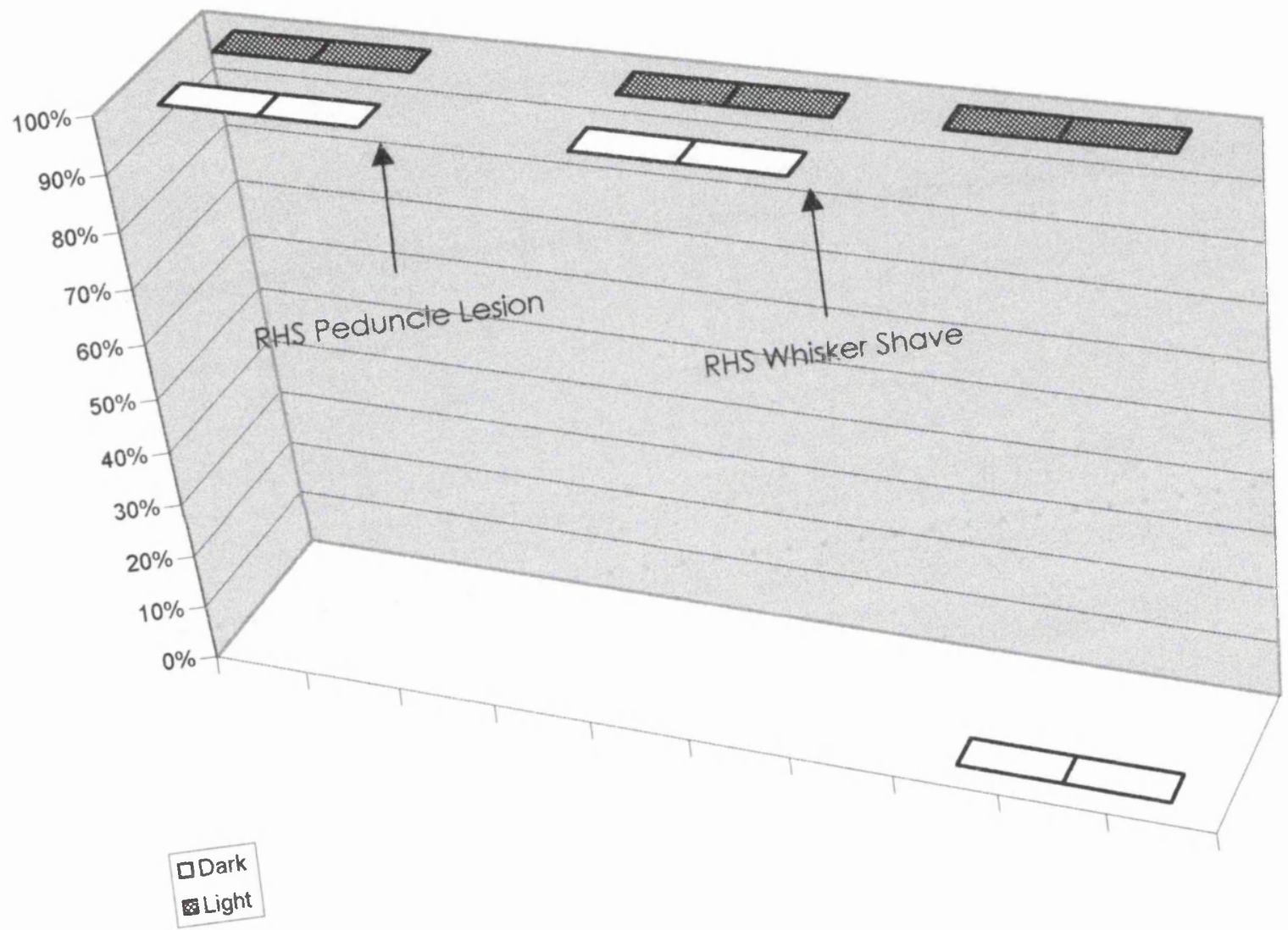

Graph showing the percentage success of the rat at a gap distance of $17 \mathrm{~cm}$. After the riskers. by cutting the right $w$ 
Fig. 23.

Case III.

Pontine Nuclei Fill.

40

42
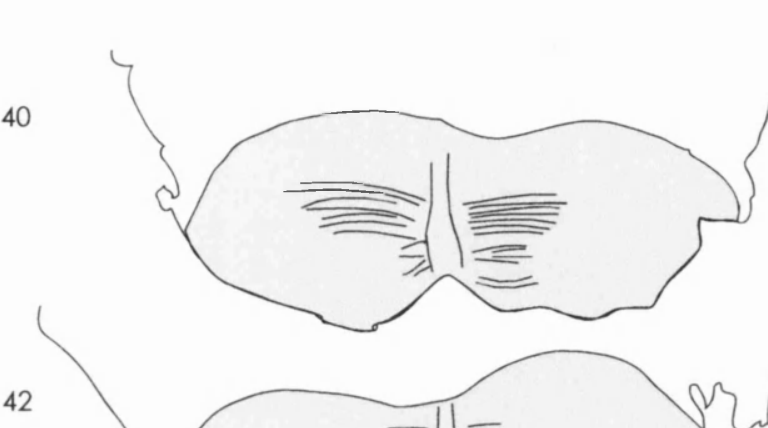

44

48

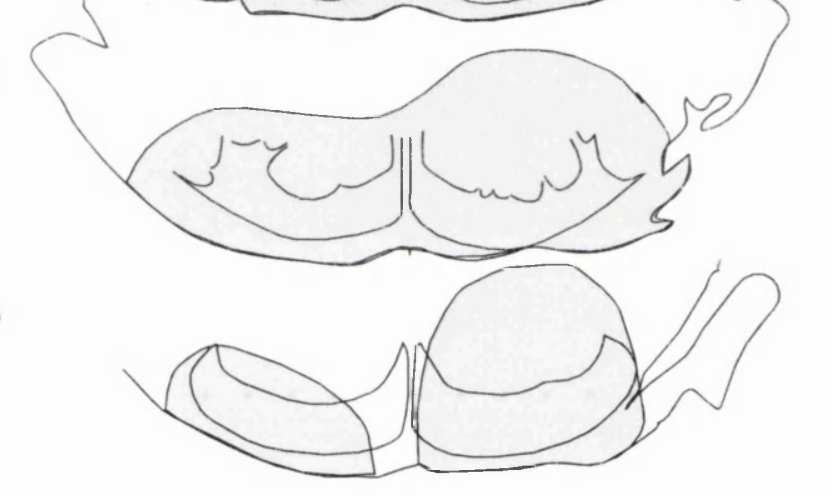

50

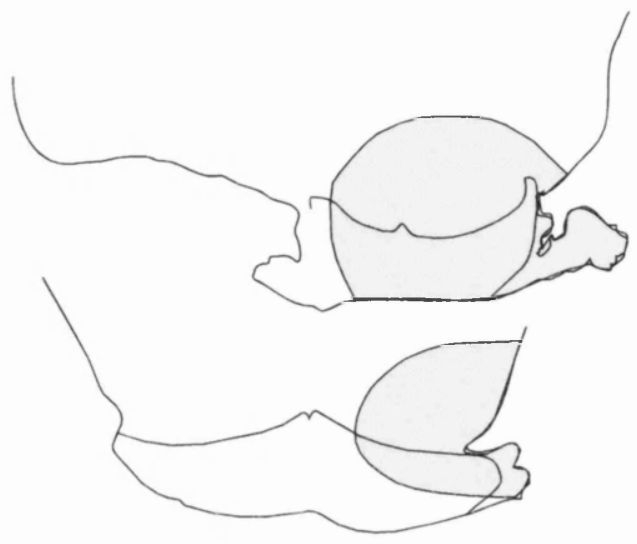

$1 \mathrm{~mm}$

Above:

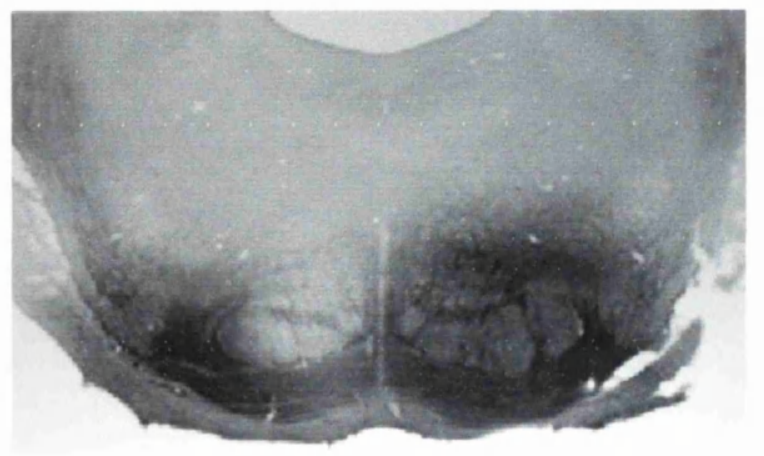

Extent of pontine nuclei injection site.

Visualised with teh chromagen D.A.B. Section 300 microns apart.

Left:

Photomicrograph of injection site. Section 46. 
Figure 24 .

Case III.

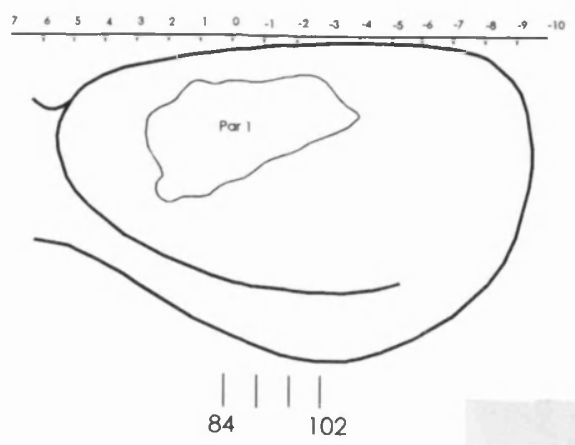

84

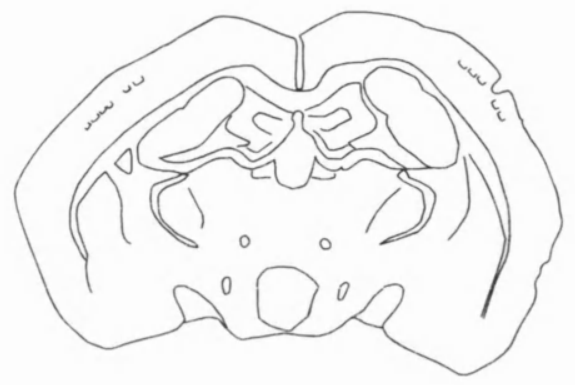

85

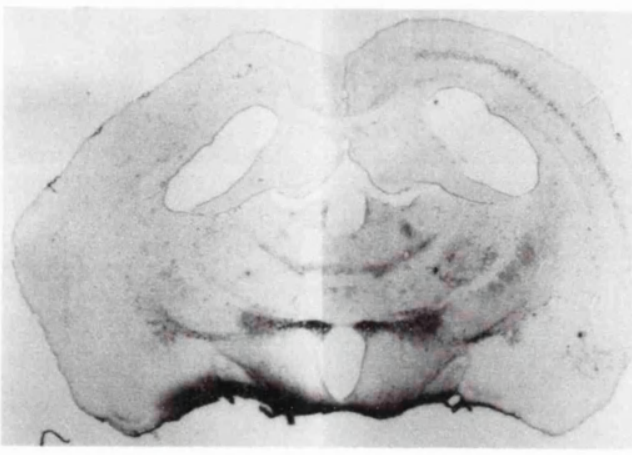

90

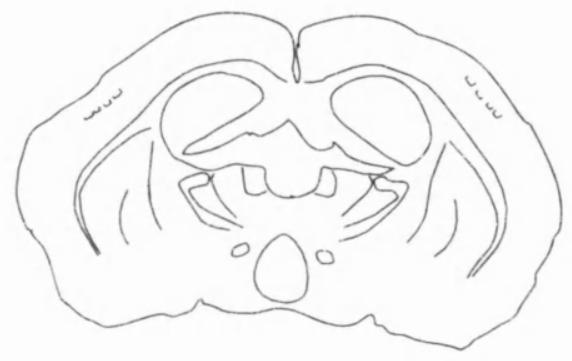

91

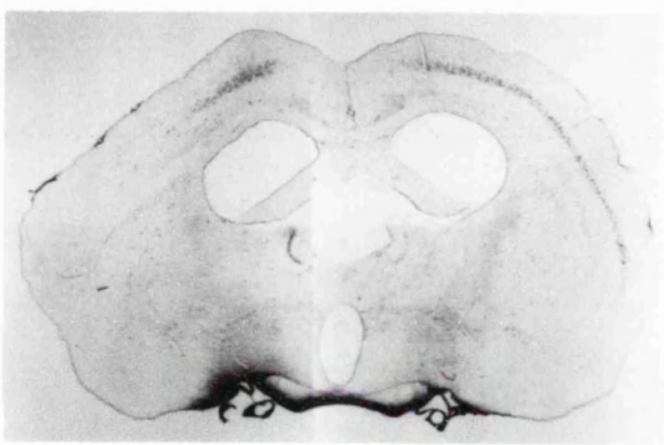

96

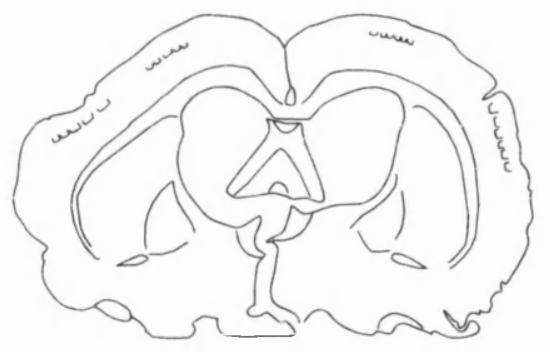

97

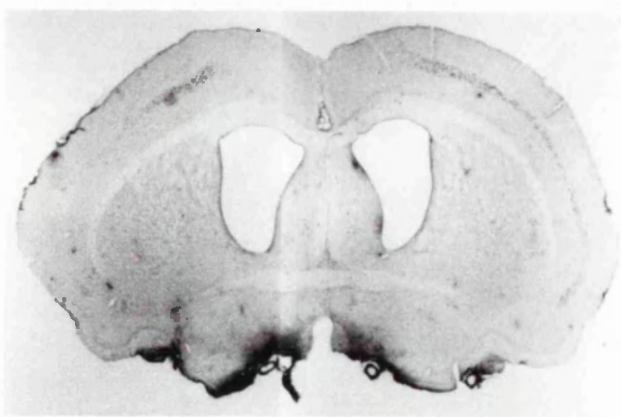

102

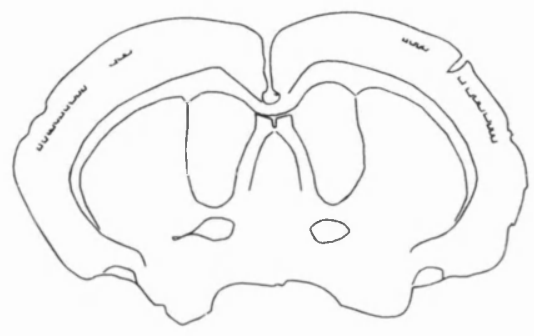

103

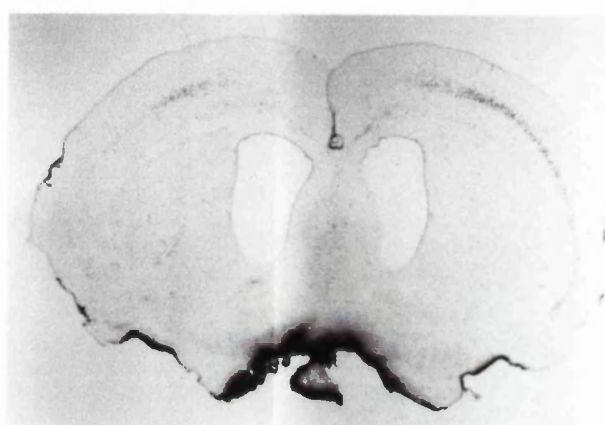




\section{Case IV}

The location of the lesion in the cerebral peduncle is shown in Figure 25. The centre of the lesion is located just rostral to the rostro-caudal middle of the peduncle, at a point level with the rostral end of the interpeduncular nucleus (Figure 25, section 39). A lesion in this position would have severed most of the efferent fibres from the PMBSF that connect the barrel fields to the pontine nucleus (Glickstein et al., 1992). The central part of the peduncle was seen to be very thin caudal to the cut fibres due to the degeneration of the fibres in this part of the peduncle. The lesion also extended into the lateral part of the substantia nigra pars reticularis.

The rat was initially trained and tested with both peduncles intact and a full complement of whiskers. The animal was then tested after the lesion had been placed in the right cerebral peduncle. Then again with a lesion in the right peduncle with only the left whiskers remaining.

Figure 26 (i) and 27 show that the rat was initially trained to jump consistently a gap of $16 \mathrm{~cm}$ in the dark using only its whiskers to gauge the distance to the far edge of the gap. At distances less than $16 \mathrm{~cm}$ the animal would use its nose, paw and vibrissae to judge the distance to the far edge.

Surgery was performed on the animal to interrupt fibres in the centre of the centre of the right cerebral peduncle. After recovery the animal was re-trained. The animal quickly regained pre-operative levels of performance. Figure 26 (ii) shows that following the lesion the animal would still cross a maximum distance of $16 \mathrm{~cm}$ in the dark 
consistently (Figure 27). The rat could be seen making contact with the far edge of the gap with its whiskers before crossing.

All the major whiskers on the right side of the face were then cut to their bases. Figure 26 (iii) shows that with only the left whiskers remaining the animal would no longer cross a gap of $16 \mathrm{~cm}$ in the dark. Figure 27 shows that the rat never crossed a gap of $16 \mathrm{~cm}$ using only its left whiskers

The distance crossed stabilised at $14 \mathrm{~cm}$ in the dark, at which distance the animal could reach across the gap with his nose.

In addition to testing in the dark, the animal was tested in the light. In the light the animal consistently jumped gaps of over $24 \mathrm{~cm}$. The ability to cross larger gaps in the light was not effected by damage to the peduncle or by cutting the right whiskers.

At the conclusion of all behavioural testing, a series of injections were made to fill the entire pontine nuclei with WGA-HRP. The injection site is shown in Figure 28. Label was found throughout the rostro-caudal extent the pontine nuclei, Although it failed to totally fill the most caudal end of the pontine nuclei (Figure 28, sections $16-20$ ). At this level the terminals of fibres from the PMBSF lie just dorsal to the fibres of the medial lemniscus (Mihailoff et al., 1985). Therefore the terminals would have been included bilaterally in the injection site in all but the very caudal end of the pons (section 16), in which case the terminals on the right side would have been included. In the rostral half of the pontine nuclei the fill was total and included all parts of the peduncle that received efferent terminals from the PMBSF (Figure 28, section $22-28$ ). 
In this case the cortex was removed, flattened and then cut parallel to the pial surface. At the level in which the sections cut through layer $\mathrm{Vb}$, retrogradely labelled cells were seen. When the left and right cortices are compared, it is obvious that the density of labelled cells is much greater in the left side of the brain (Figure $29 \& 30$ ), indicating that the had interrupted most of the fibres that connect the PMBSF to the pontine nuclei. 
Figure 25.

CaselV.

Cerebral Peduncle Lesion.

35

36

37

38

39

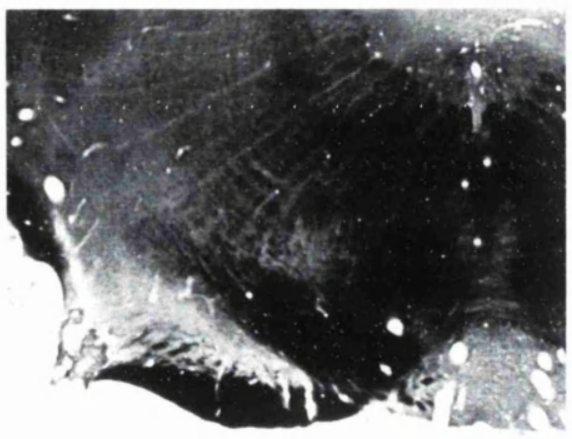

40

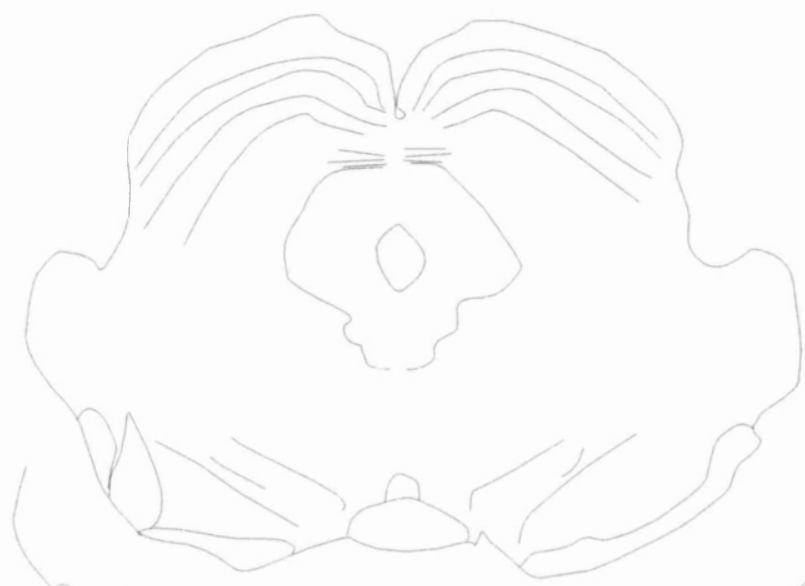

42

43

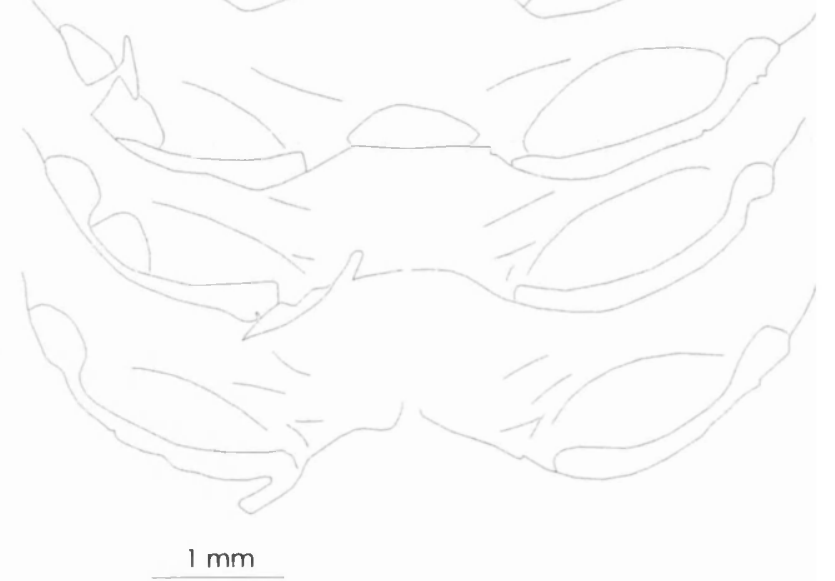

Above:

Lesion in the cerebral peduncle. Sections 150 microns apart.

Left:

Photomicrograph of lesion. Section 37 
Figure 26.

Case IV.
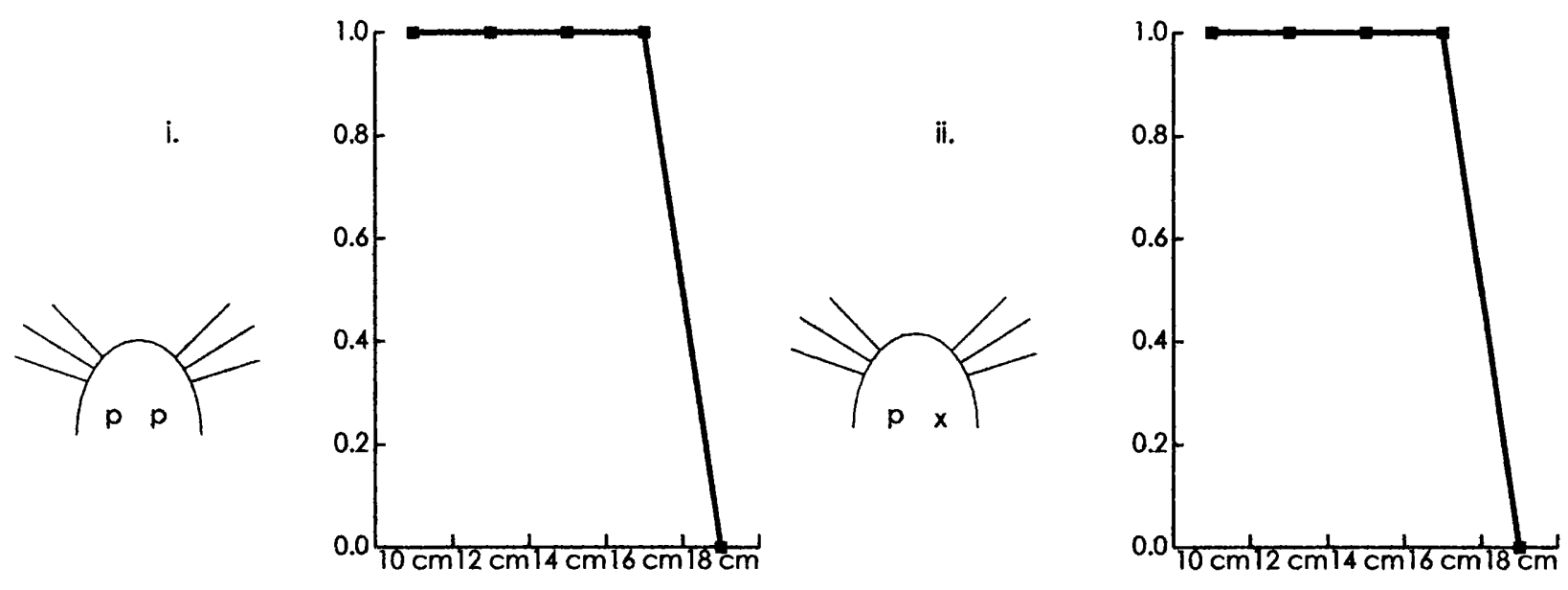

\section{Peduncles and whiskers intact}

Right peduncle cut Whiskers intact
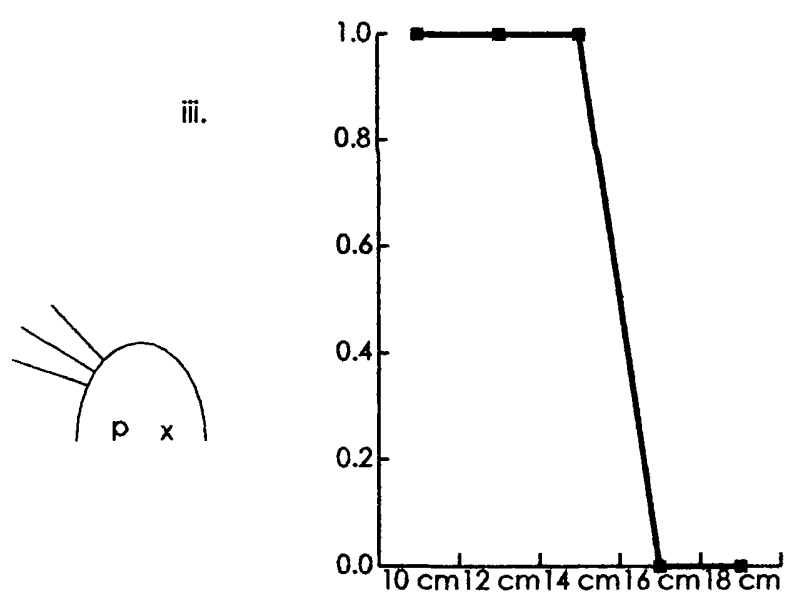

Right peduncle lesion

Right whisker shave

Graphs showing the probability of the rat crossing at differing gap distances in its normal state (i), with a lesion in the right peduncle and both sets of whiskers (ii), and with a lesion in the right peduncle and only the left whiskers (iii). 
Figure 27.

Casse IV.

\section{$\%$ Success At $16 \mathrm{~cm}$}

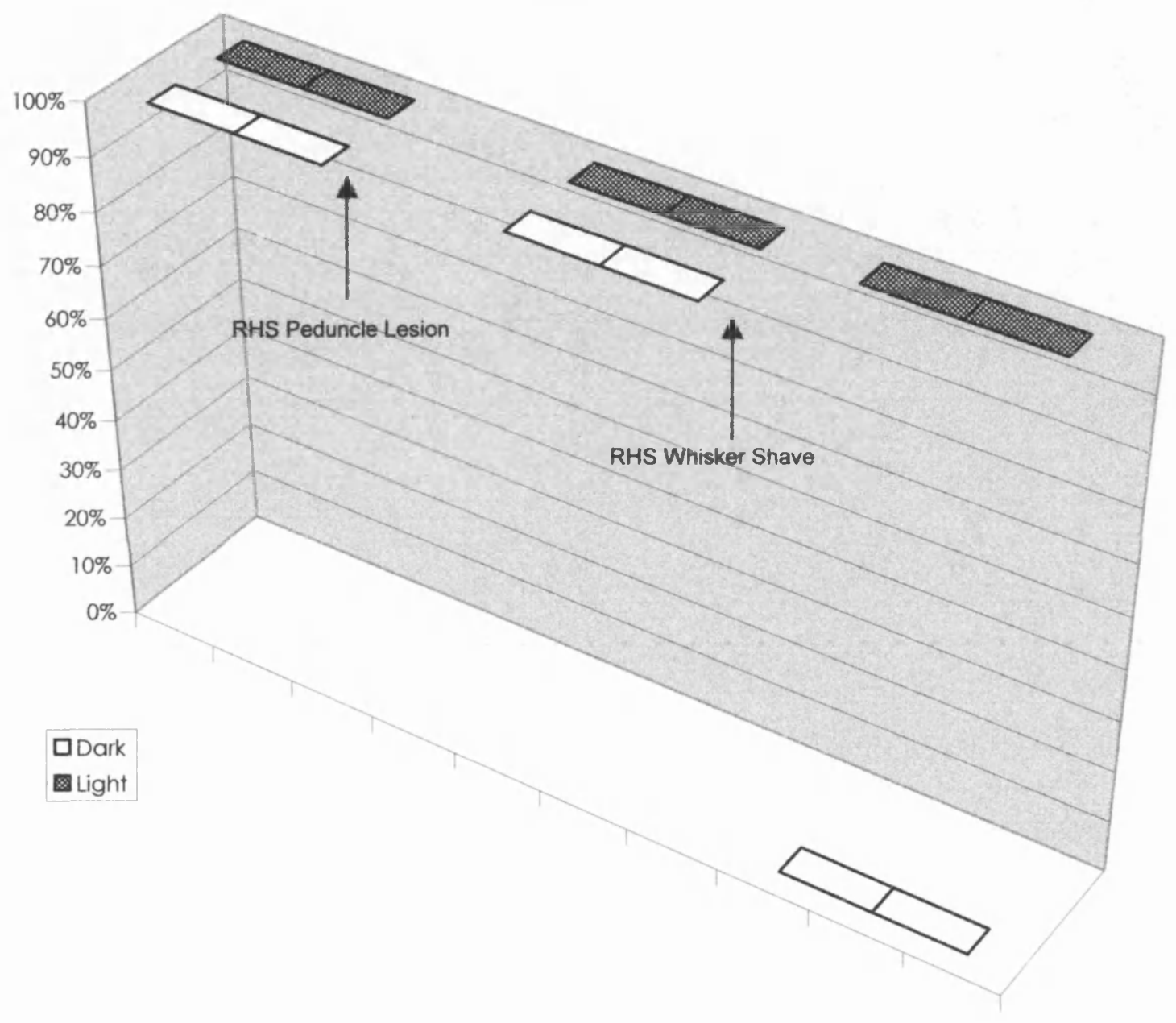

Graph showing the percentage success by the rat at a gap distance of $16 \mathrm{~cm}$, after a right peduncle lesion followed by cutting the right whiskers. 
Figure 28.

Case IV.

Pontine Nuclei Fill.

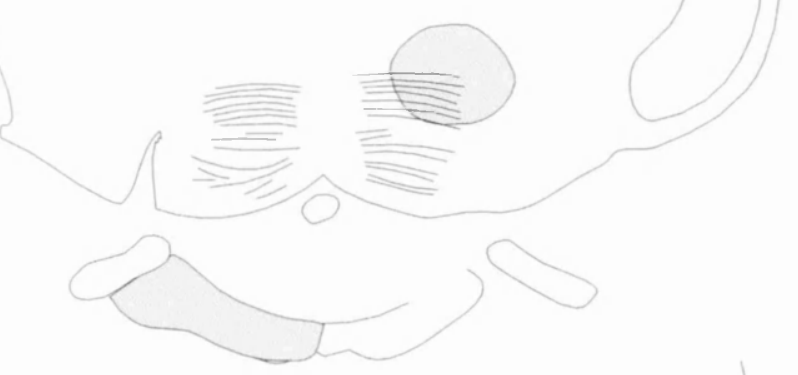

22

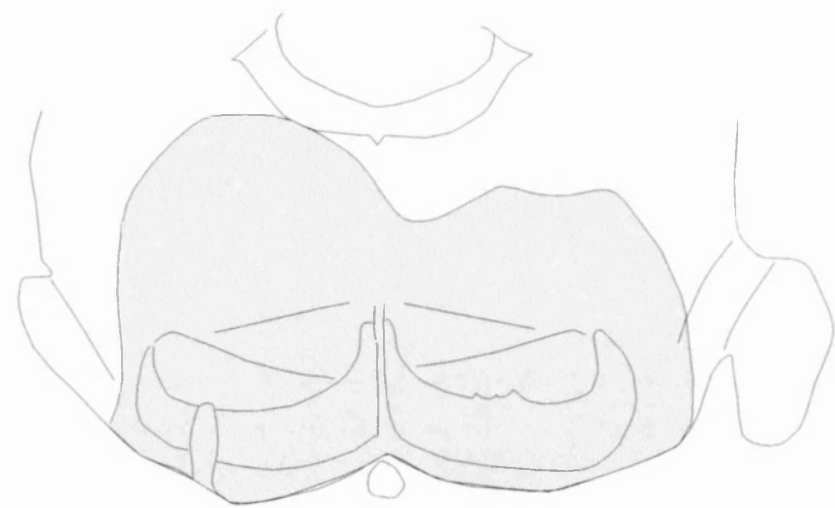

24

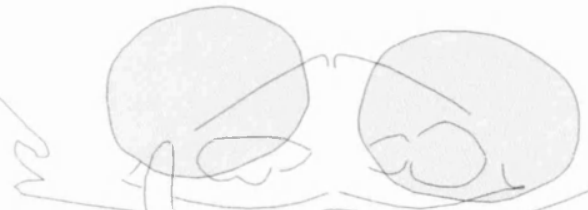

26
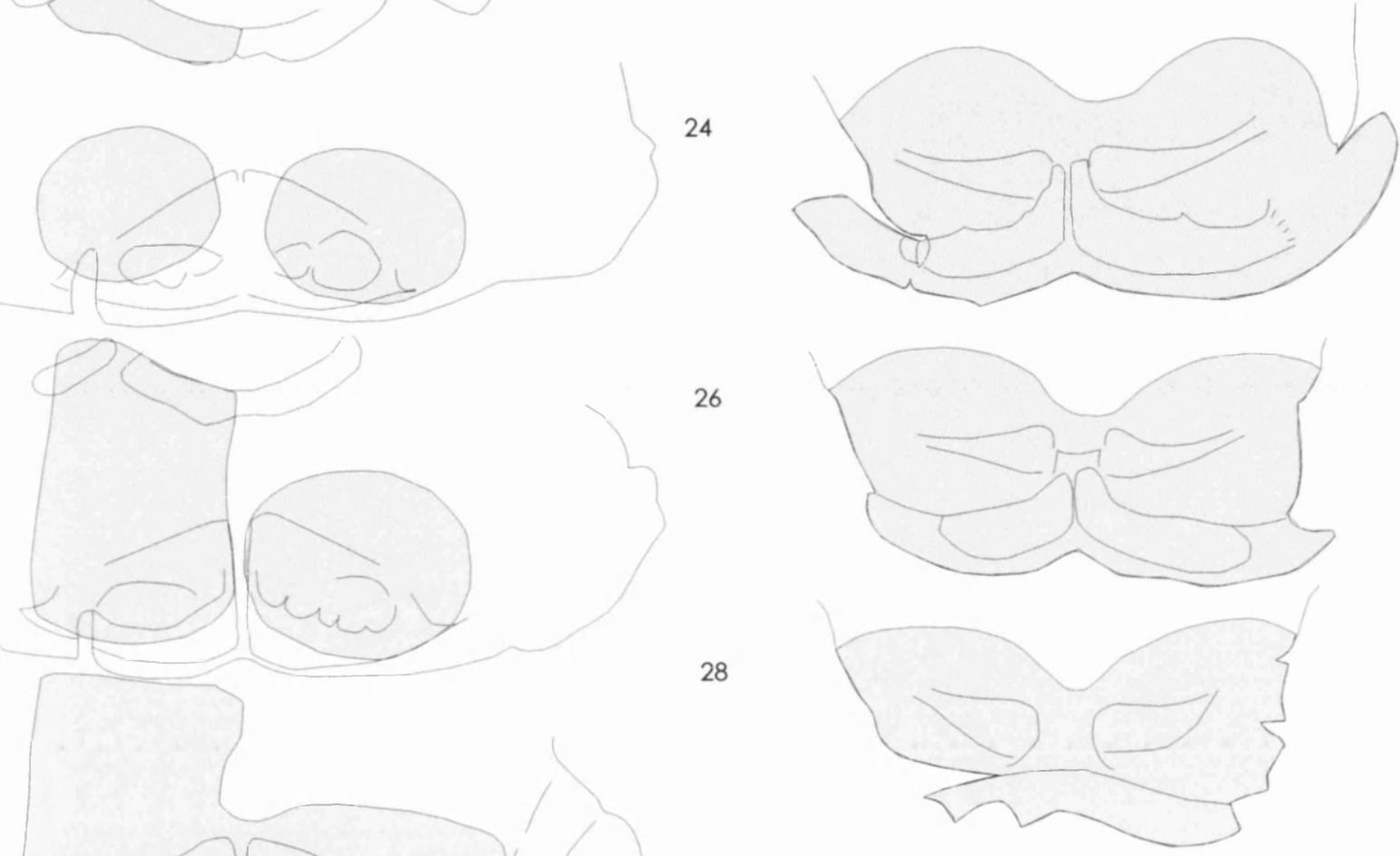

$1 \mathrm{~mm}$

Above:

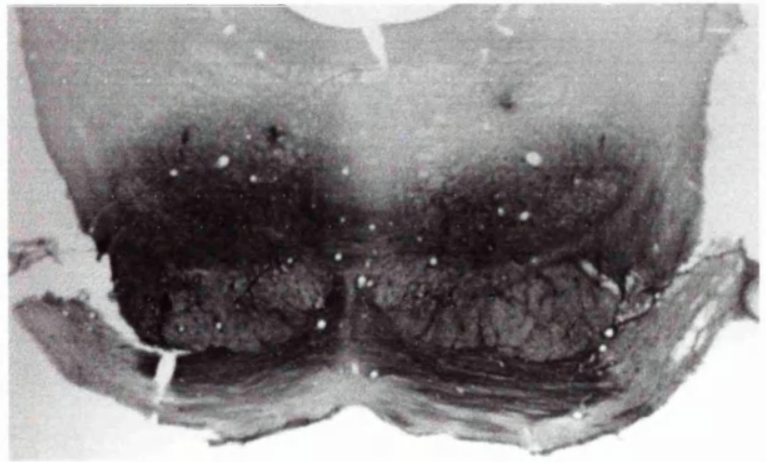

Injection site in the pontine rivclei reacted with $D A B$. Sections are 300 microns apart.

Left:

Photomicrograph of injection site. Section 24. 
Figure 29.

Case IV.

L.H.S.
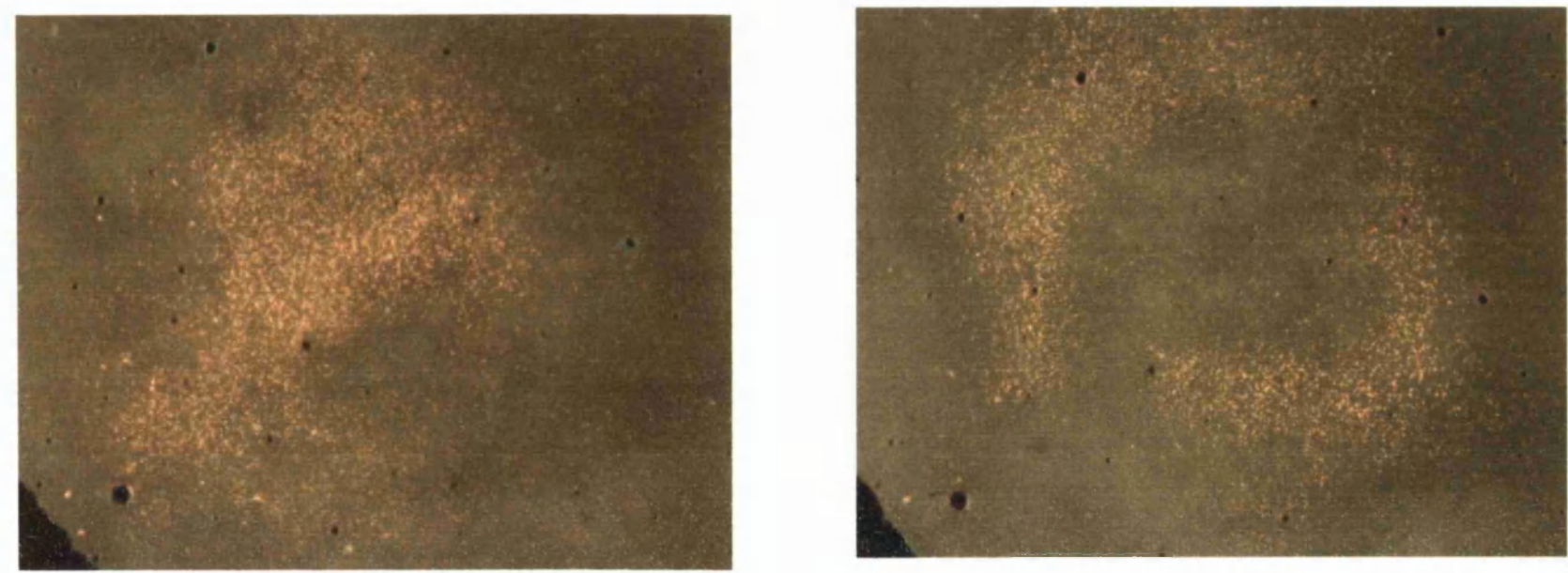

Above: Consecutive sections through layer $\mathrm{Vb}$ of the left flattened P.M.B.S.F. of Case IV showing H.R.P. labelled cell bodies. Stars indicate the vessels used as markers to line up the sections for the montaged figure (below).

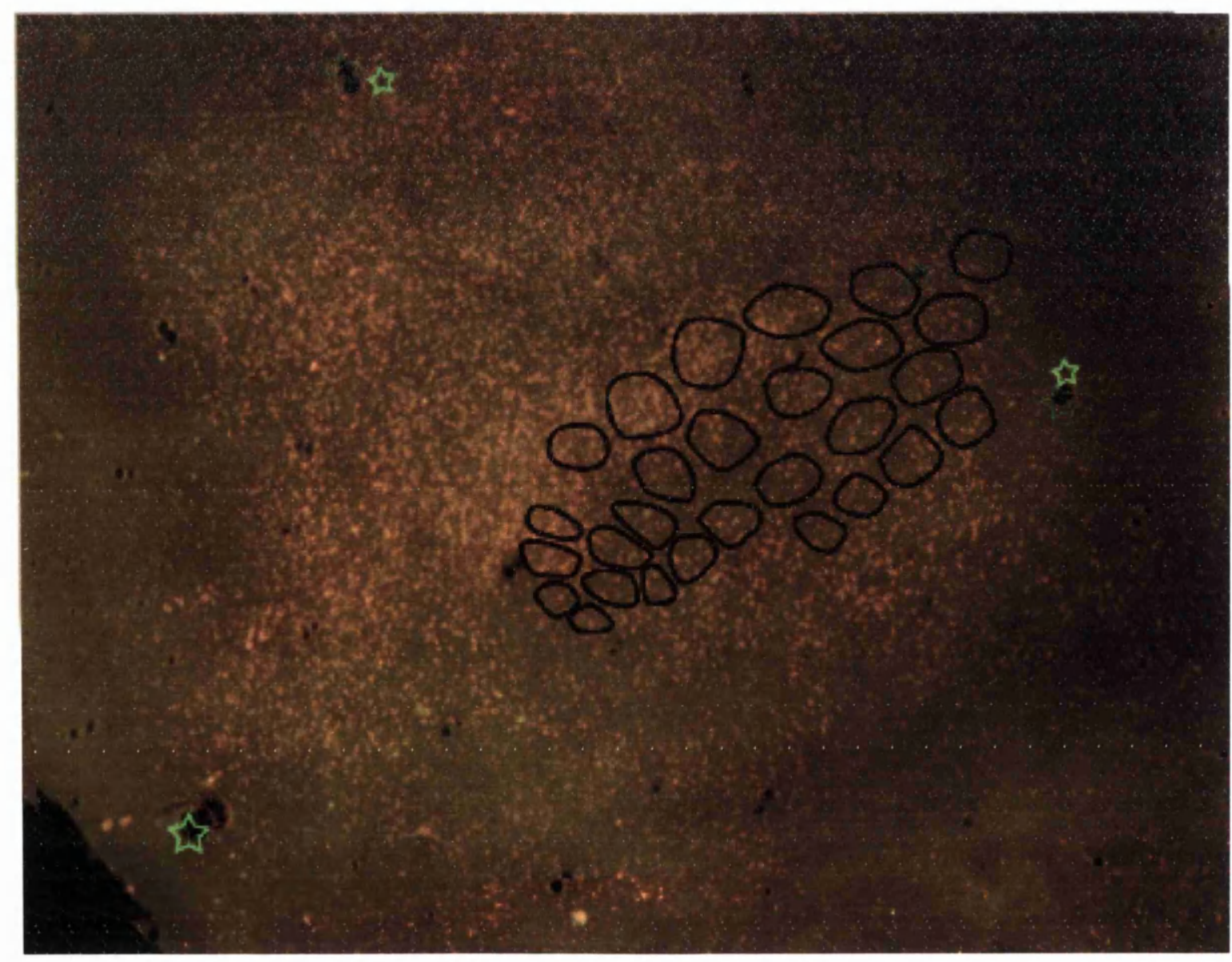

Above: a composite of the top photographs. Superimposed, is a drawing of the P.M.B.S.F created from flattened sections through layer IV stained to reveal cytochrome oxidase. Stars represent the vessels in used to align the individual sections. 

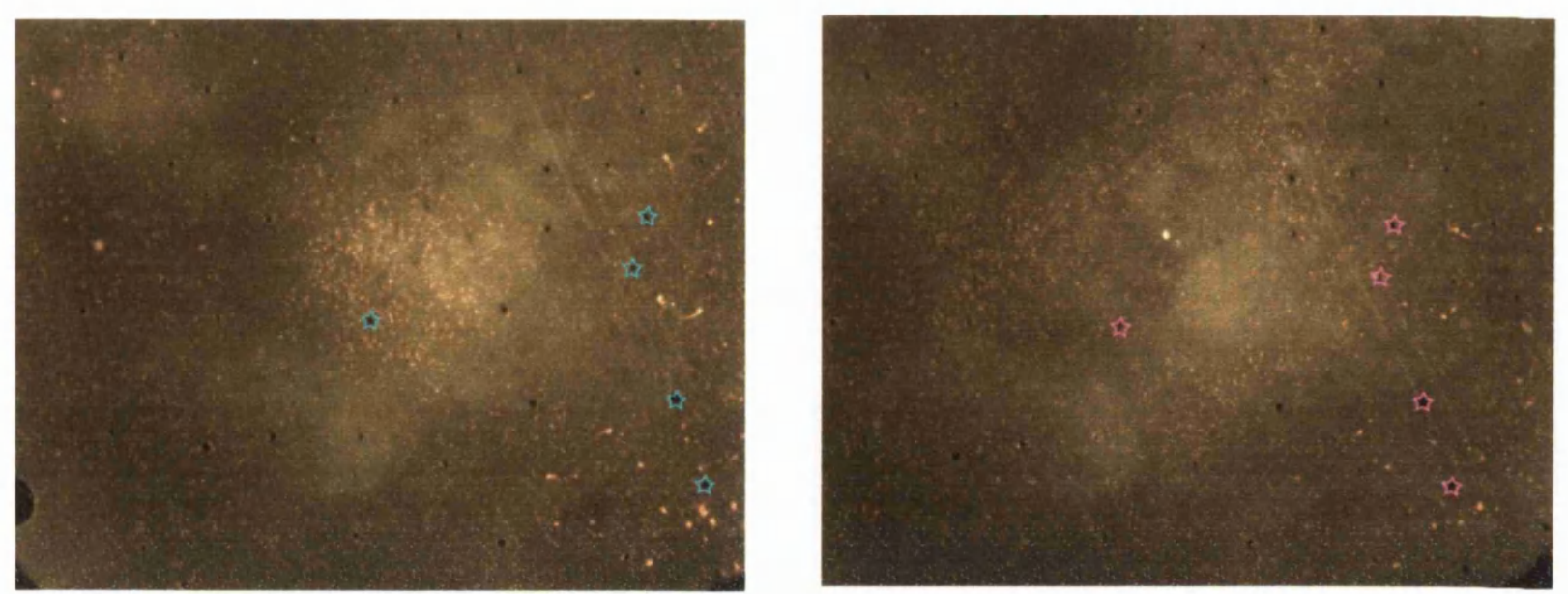

Above: Consecutive sections through layer Vb of the right flattened P.M.B.S.F. of Case IV showing H.R.P. labelled cell bodies. Stars indicate the vessels used as markers to line up the sections for the montaged figure (below).

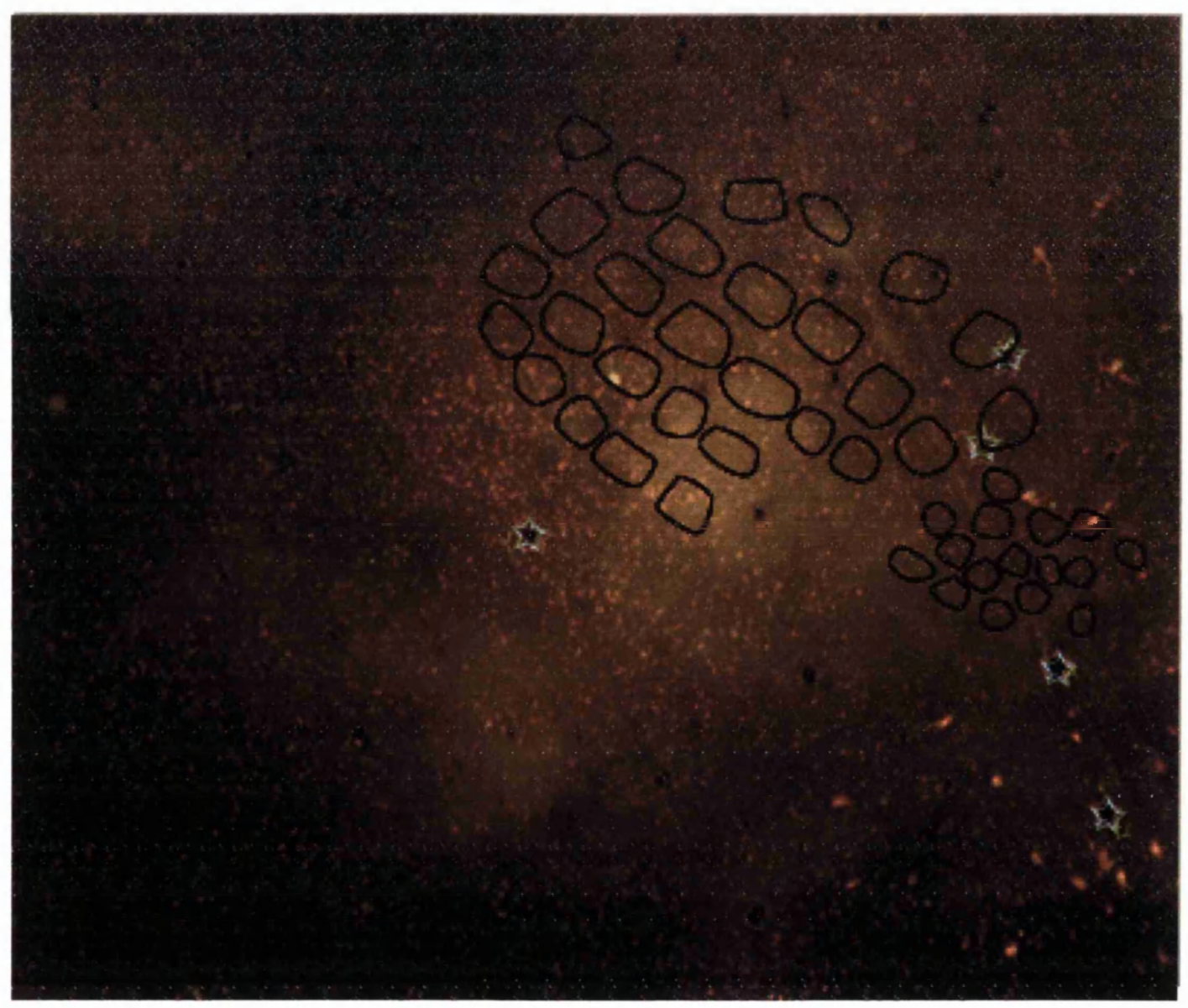

Above: a composite of the top photographs. Superimposed, is a drawing of the P.M.B.S.F created from flattened sections through layer IV stained to reveal cytochrome oxidase. Stars represent the vessels in used to align the individual sections. 


\section{Case V}

The lesion to the right cerebral peduncle is shown in Figure 31. The lesion is placed just caudal to the middle of the peduncle in its rostro-caudal extent, and just dorsal to its centre. In sections through the peduncle taken above the lesion (Figure 31, sections 7678) the cerebral peduncle is seen to be missing degenerated fibres In the middle section. These fibres correspond to the position of those shown to contain labelled fibres following an injection of WGA-HRP into the PMBSF (Glickstein ef al., 1992). The lesion encroached into the lateral part of the substantia nigra pars reticularis.

The animal was first trained and tested as normal with full sets of whiskers and both peduncles intact. Then with all whiskers and a lesion in the right peduncle. Then finally with the lesion in the right peduncle and only the left set of whiskers.

Figure 32 (i) and 33 show that the animal was originally trained to consistently cross a gap of $16 \mathrm{~cm}$ in the dark. At $16 \mathrm{~cm}$ the animal could only reach the far side of the gap with its whiskers to judge the distance to jump. At gaps of $14 \mathrm{~cm}$ and below the animal used a mixture of vibrissae, paw and nose to judge the distance.

When performance was stable surgery was performed to cut the central fibres of the right cerebral peduncle. After recovery from the surgery the animal was re-tested. The animal quickly re-gained preoperative levels of performance (Figure 32, ii). With both sets of whiskers intact the lesion had no effect on the animals ability to judge the gap distance to cross in the dark. 
At this point all the whiskers on the right side of the face were cut to their bases. Figure 32 (iii) shows that the maximum distance the rat would cross in the dark using only its left whiskers was reduced by $2 \mathrm{~cm}$ to $14 \mathrm{~cm}$. Figure 33 shows that the rat did not once cross a gap of $16 \mathrm{~cm}$, although the remaining left whiskers could be seen touching the far edge of the gap. The rat used its nose and paw to judge gap distances of $14 \mathrm{~cm}$ and below before crossing.

During all the testing in the dark the rat was tested in the light and would consistently crossed a gap of $26 \mathrm{~cm}$ regardless of the condition of the right peduncle or the vibrissae.

The fibres cut by the lesion in the cerebral peduncle were confirmed by the distribution of terminal label in the pontine nuclei following bilateral injections of WGA-HRP into the vibrissal-barrel fields. Similar quantities of WGA-HRP were injected into both vibrissalbarrel fields. The injections were centred in roughly the same part of the PMBSF in either hemisphere, though the injection in the right hemisphere had a larger spread than that in the left (Figure $34 \& 35$ ).

Although the injection in the right PMBSF was larger than that in the left, the amount of terminal label in the right side of the pontine nuclei is considerably less than the amount of label seen in the left (Figure 36).

The position of the lesion, the bias of the terminal label toward the side of the pontine nuclei which was connected to the cortex with an intact cerebral peduncle suggests that most, if not all of the fibres linking the PMBSF to the pontine nuclei on the right side of the brain had been cut. 
The animal was tested for orientation responses both before and after the lesion in the cerebral peduncle. There was no apparent difference in the animal's behaviour after the lesion. 
Peduncle Lesion.
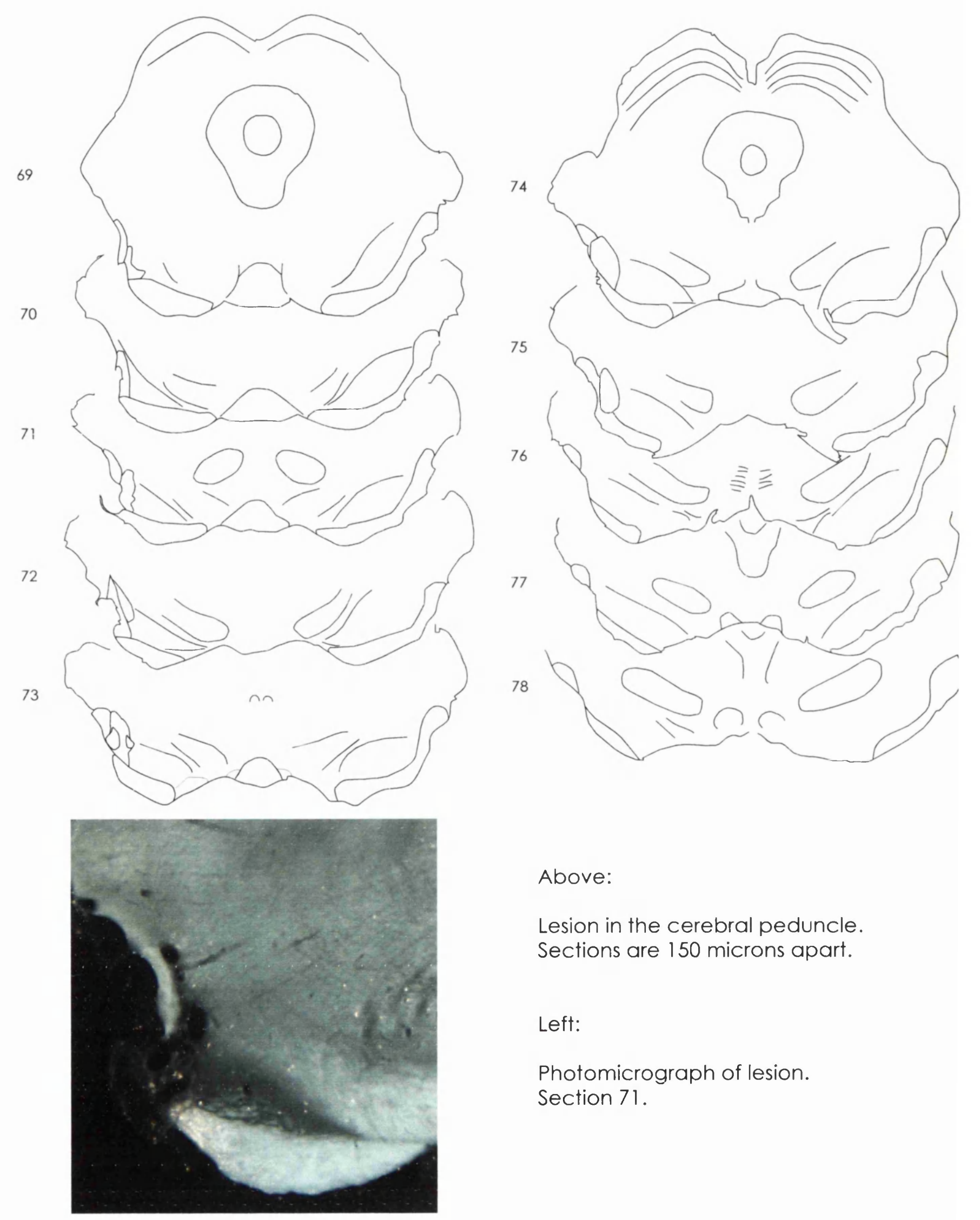

Above:

Lesion in the cerebral peduncle. Sections are 150 microns apart.

Left:

Photomicrograph of lesion. Section 71. 
Figure 32 .

Case V.
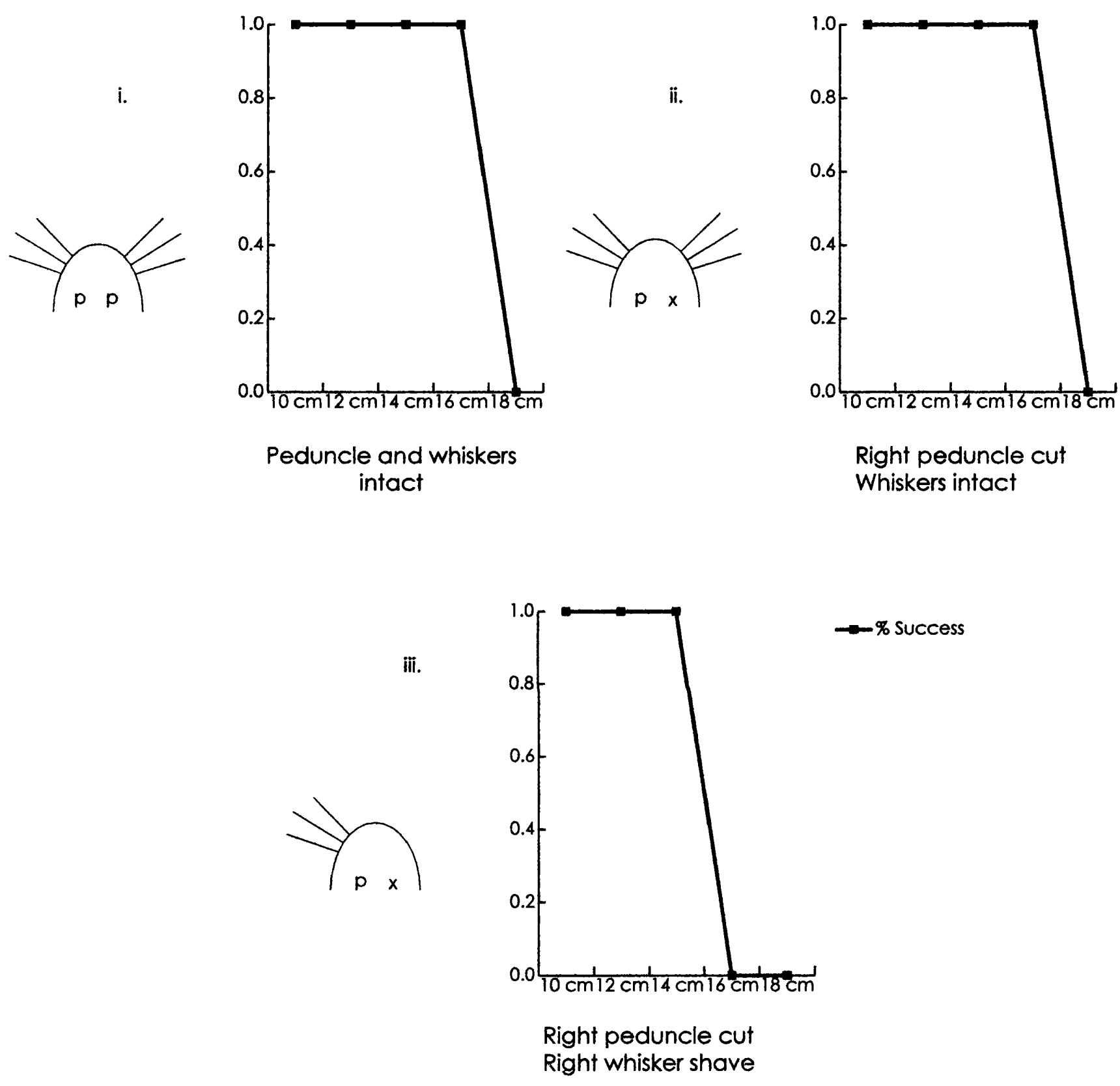

Graphs showing the probability of the rat crossing at differing gap distances in its normal state (i), with a lesion in the right peduncle and both sets of whiskers (ii), and with a lesion in the right peduncle and only the left whiskers (iii). 

Figure 34.

Case V.

WGA-HRP injection.

RHS.

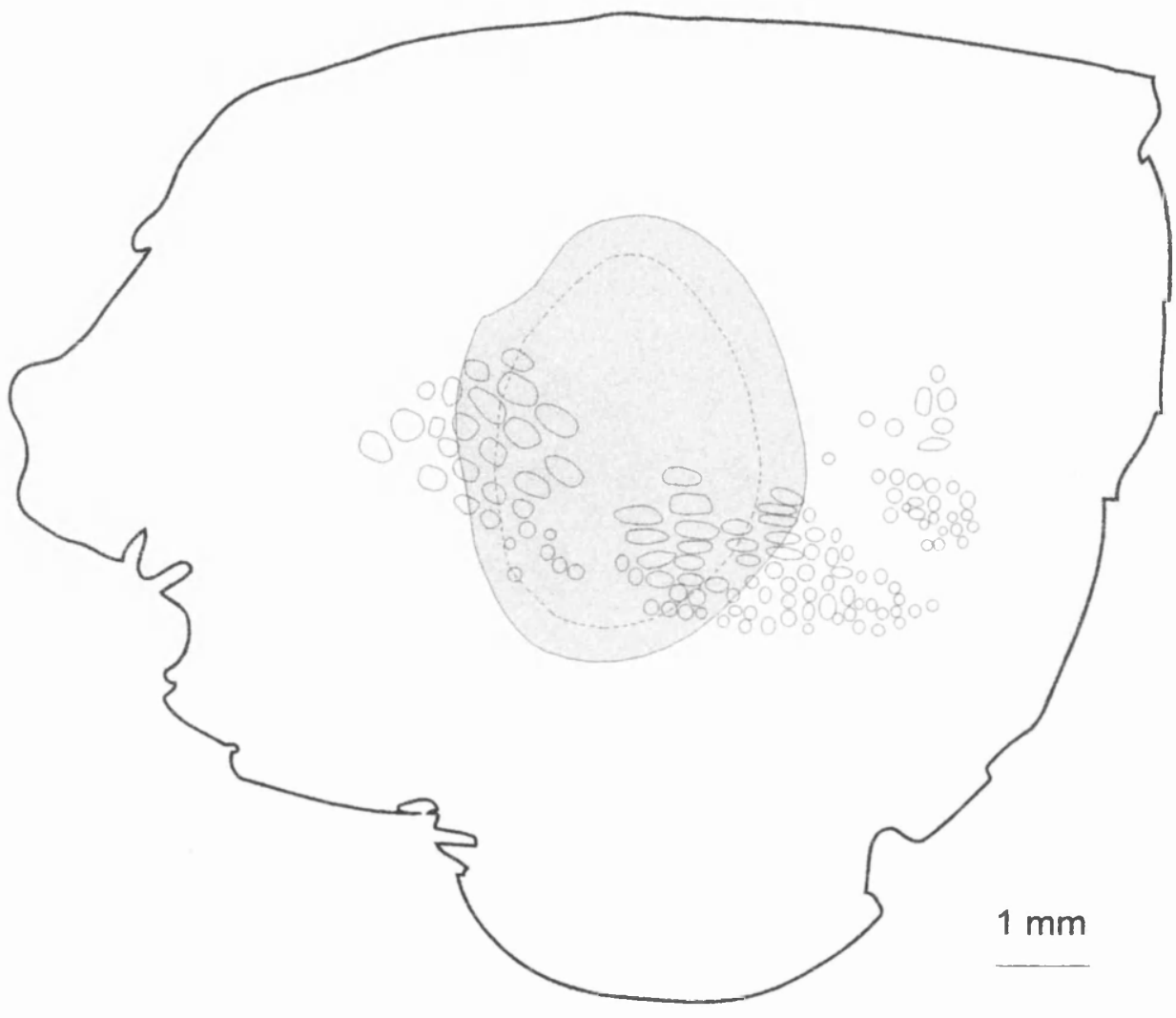

Flatened cortex through the PMBSF of the right hemisphere. The shaded area represents the extent of the WGA-HRP injection site. 
Figure 35.

Case V.

WGA-HRP Injection.

LHS.

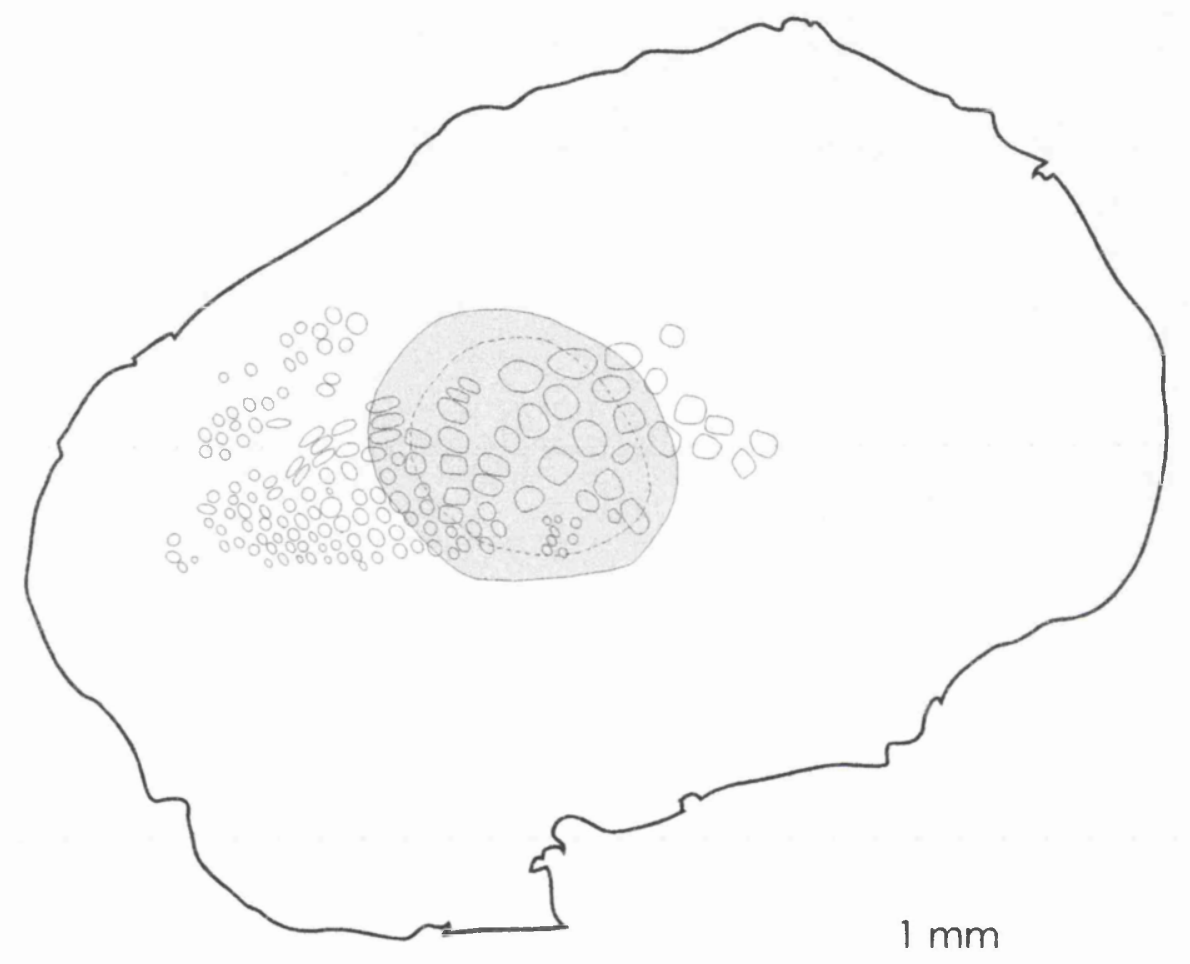

Flatened cortex through the PMBSF of the the left hemisphere. The shaded area represents the extent of the WGA-HRP injection site. 
Figure 36.

Case V.

Pontine Nuclei Label.

52

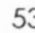

54

55

56

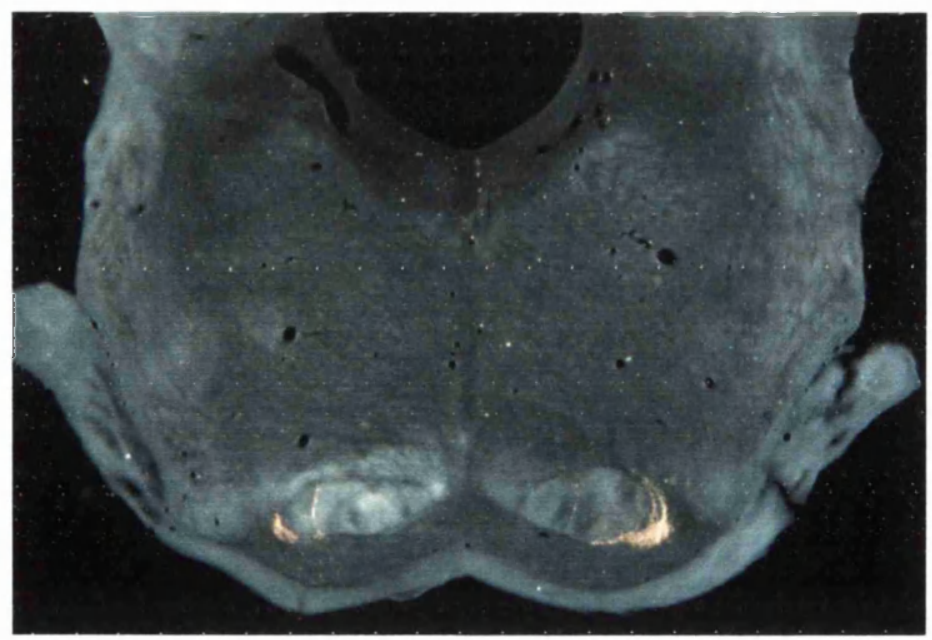

57

58

59

60

61

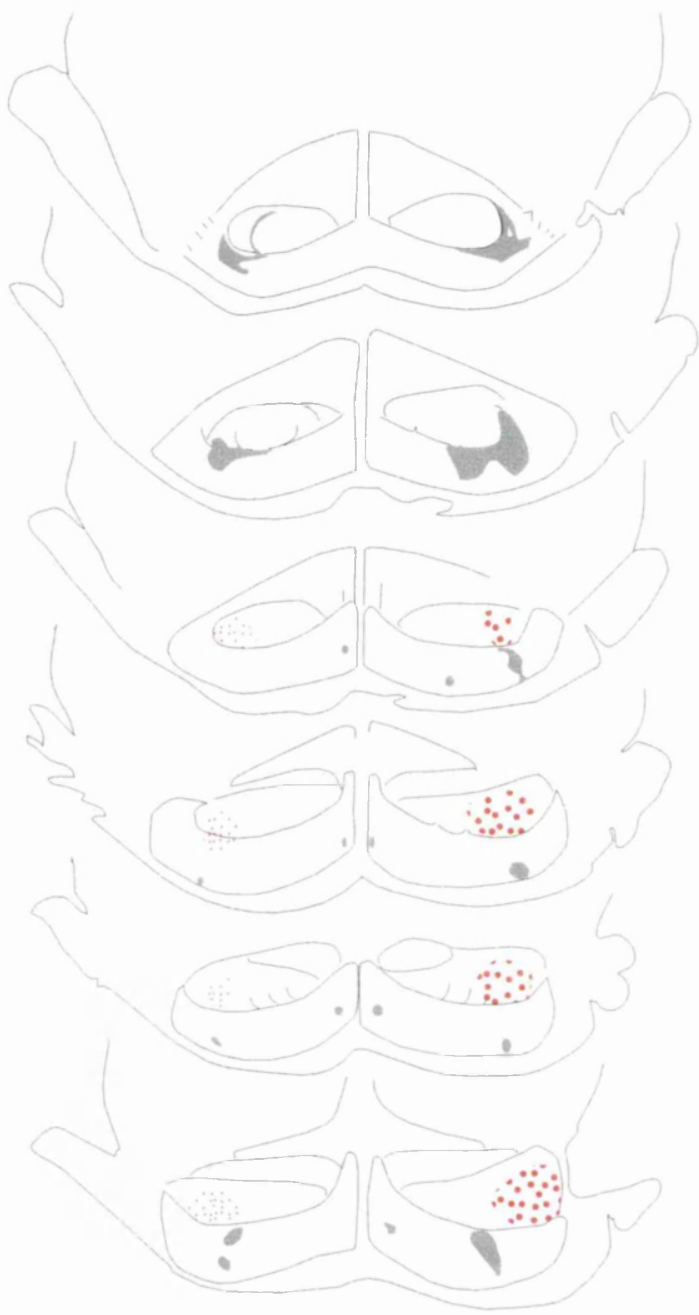

Above:

Pontine label after injection of WGA-HRP bilaterally into the

PMBSF.

Sections 150 microns apart.

Left:

Photomicrograph of pontine nuclei. Section 57. 


\section{Case VI}

The peduncle lesion in this case is shown in Figure 37. The lesion is centrally placed in both the rostro-caudal and the dorso-ventral axes of the peduncle. In this position it will have destroyed many of the descending efferent fibres from the vibrissal-barrel field (Glickstein et al., 1992). With a small encroachment into the lateral part of the substantia nigra pars reticularis.

The animal was tested first pre-operatively with its full complement of cerebral peduncles and whiskers. It was re-tested with all whiskers following a lesion in the right peduncle, then with the right peduncle lesion and the left whiskers shaved. Finally the left whiskers were allowed to re-grow and then the right whiskers were shaved.

Figure 37 shows the behavioural results in sequence. With the peduncles and whiskers intact the animal was trained to jump across the gap at a stable criterion distance of 16 $\mathrm{cm}$ in the dark (Figure 38, i). At this distance the rat used only its whiskers to gauge the length of the gap. At gaps of $14 \mathrm{~cm}$ and less the animal was able to use its whiskers, nose or paw to gauge the gap. At gaps larger than $16 \mathrm{~cm}$ the animal could not reach the far side with any part of its body and always refused to cross this distance in the dark.

After performance stabilised the central part of the right cerebral peduncle was ablated. After recovery the animal was re-tested. At this point the animal had both sets of whiskers but only the left cerebral peduncle intact. 
Figure 38 (ii) shows that the animal would still invariably cross gaps of distances up to and including $16 \mathrm{~cm}$, using his whisker to reach the far edge at a gap of $16 \mathrm{~cm}$, and a combination of vibrissae paw and nose at smaller gaps. With both sets of whiskers intact, the unilateral peduncle lesion alone had no affect on the animal's performance in this task.

A this point the whiskers on the left side of the face were shaved to their bases. Figure 38 (iii) shows that with a lesion in the right peduncle and the ipsilateral whiskers the animal behaved as it had done when it had a full complement of whiskers, and used the remaining whiskers to judge the gap distance. Once the animals behaviour was shown to be stable testing was stopped and the animal rested after its whiskers had regrown.

When the animal had full sets of whiskers the animal was re-tested. Performance remained at the same levels as seen previously.

The right whiskers were then cut to their bases. Figure 38 (iv) and 39 shows that this produced an abrupt change in the levels of performance. The rat immediately stopped crossing the gap of $16 \mathrm{~cm}$ in the dark, even thought the remaining left whiskers could be seen to touch the far side of the gap. The rat initially would only cross a gap of $12 \mathrm{~cm}$ in the dark, but stabilised over the next seven trials at a distance of $14 \mathrm{~cm}, 2$ $\mathrm{cm}$ less than he could cross previously. At this distance the animal gauged the gap width using the tip of his nose or his paw before crossing. 
Throughout all the testing in the dark the animal was also tested in the light. The animal consistently crossed gaps of $32 \mathrm{~cm}$ in the light regardless of the state of either the vibrissae or the cerebral peduncles.

At the conclusion of all of the behavioural testing, verification that the lesion had destroyed fibres connecting the PMBSF to the pontine nuclei was given by plotting retrogradely labelled cells following large injections of WGA-HRP to fill the entire pontine nuclei. Eight injections of WGA-HRP were made into the brainstem to fill the entire pontine nuclei. The injection site fills the entire pontine nuclei entirely and is shown in Figure 40.

At the level of the vibrissal barrel field retrograde label was heavily biased toward the side of the brain in which the cerebral peduncle had not been lesioned (Figures 41, 42, 43, $44 \& 45)$. On the side of the brain with an unlesioned peduncle at the level of the PMBSF retrogradely labelled cells can be seen in their normal distribution throughout layer $\mathrm{Vb}$. On the side of the lesioned peduncle only a few labelled cells were present in layer $\mathrm{Vb}$. In cortical areas outside of the PMBSF, such as the hind, forelimb and secondary sensory areas as well as frontal cortex there was the expected distribution of cells bilaterally.

The rat's orientation responses were tested before and after it had received a lesion in the cerebral peduncle. There was no difference in the animals behaviour following surgery. 
Figure 37.

Case VI.

Peduncle Lesion.
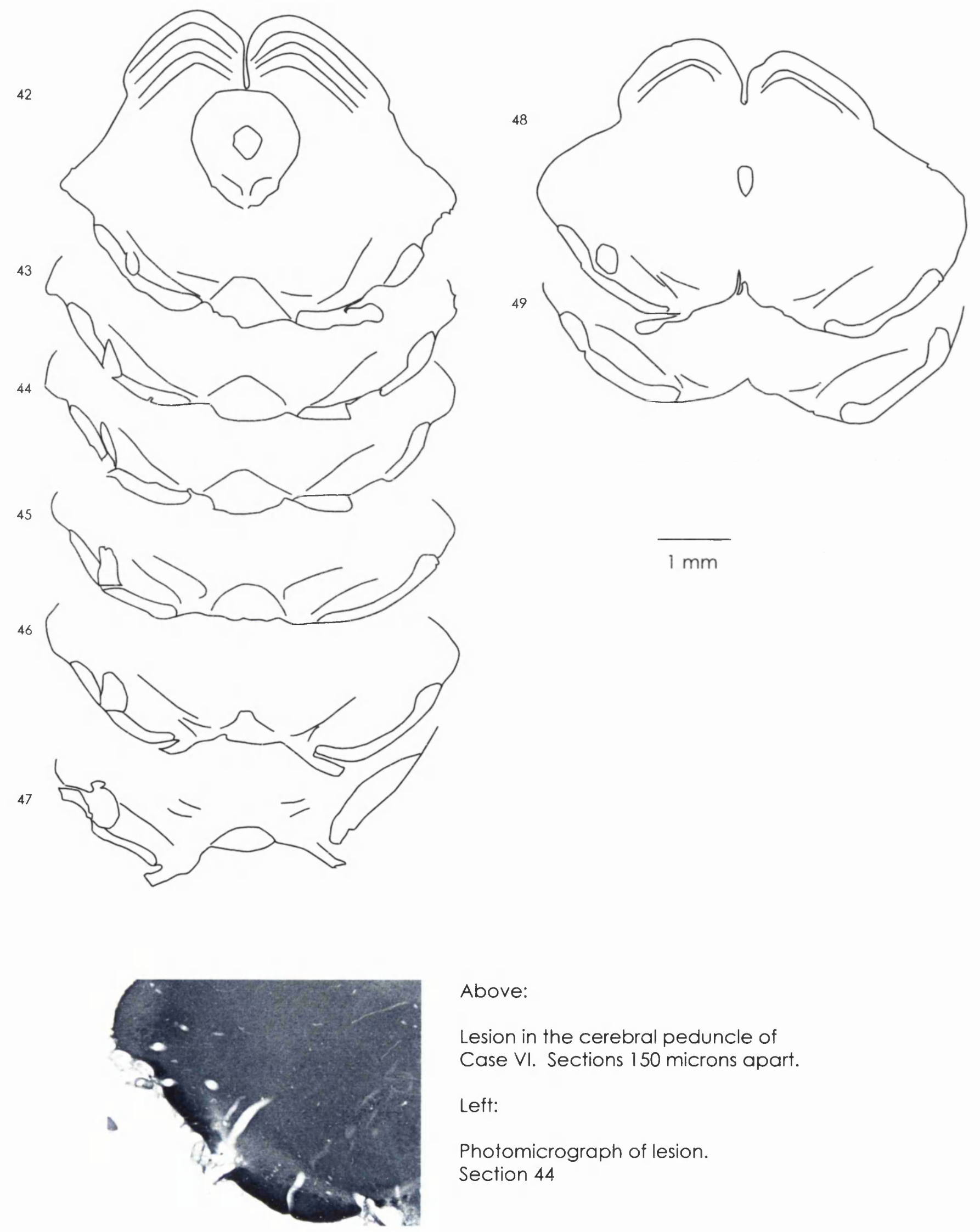

Above:

Lesion in the cerebral peduncle of

Case VI. Sections 150 microns apart.

Left:

Photomicrograph of lesion.

Section 44 
Figure 38 .

Case VI.
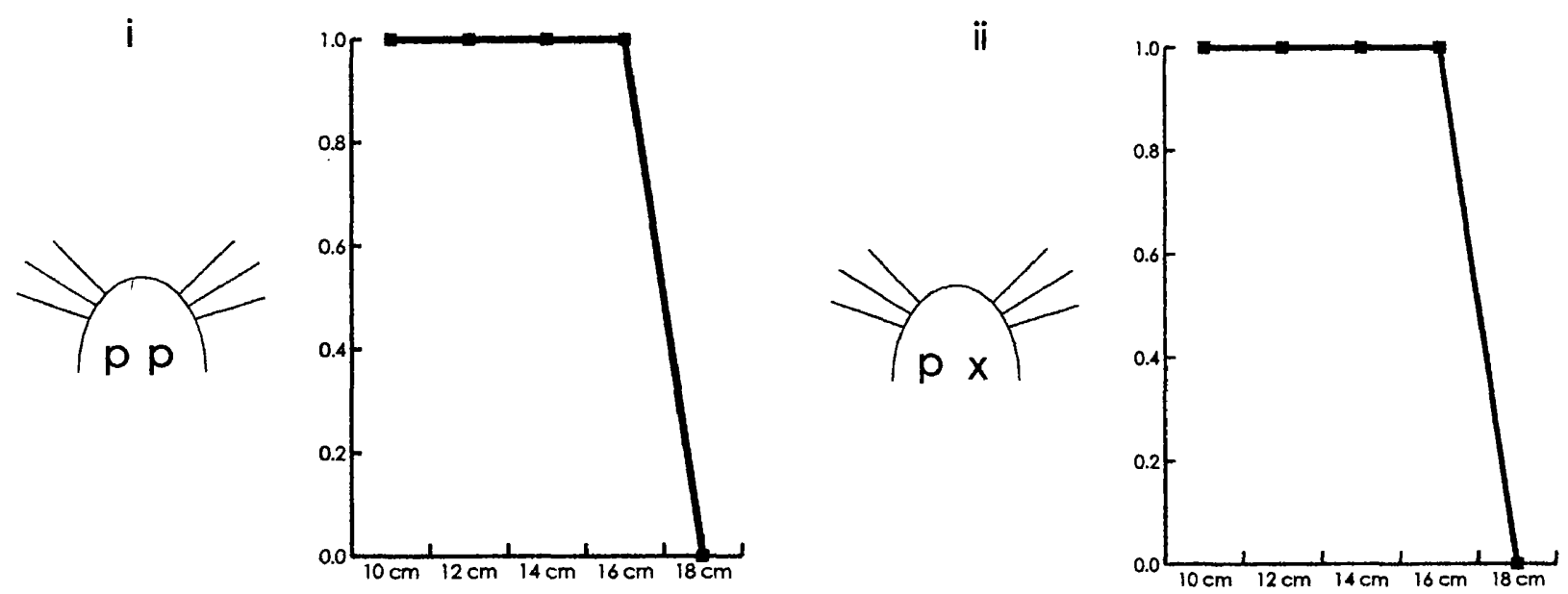

Intact

Right peduncle lesion
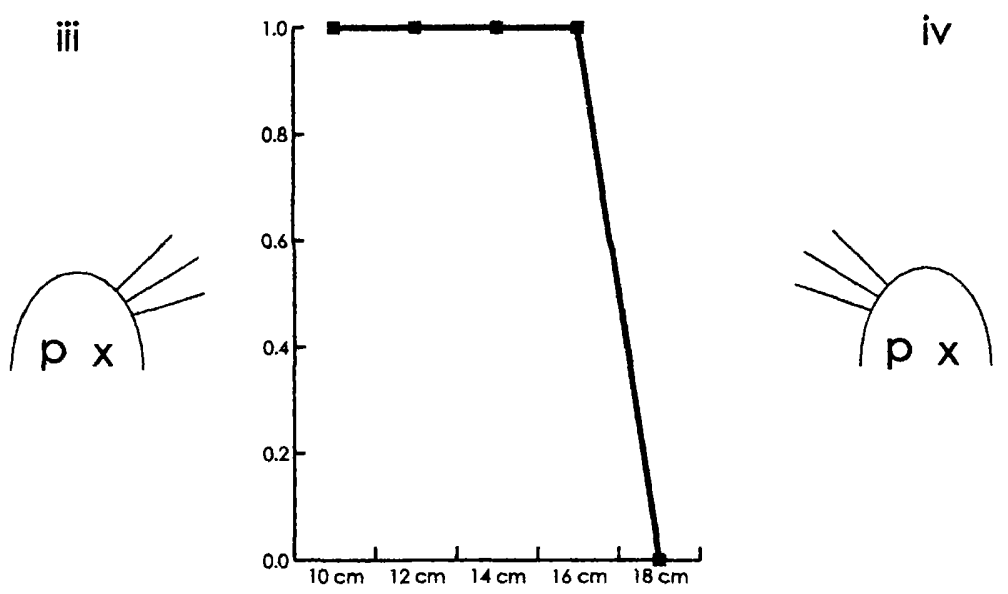

Right peduncle lesion Left whisker shave

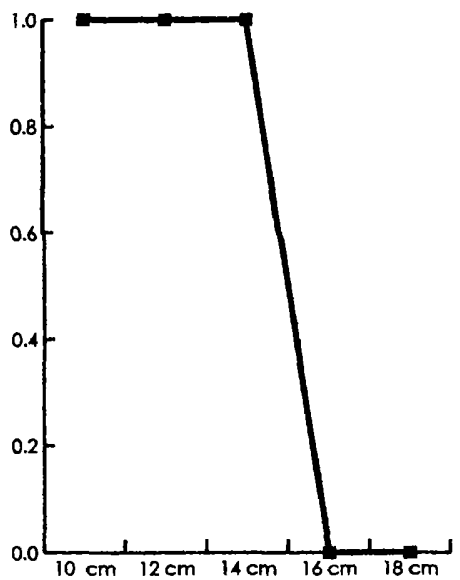

Right peduncle lesion Left whisker re-growth Right whisker shave

Key:
$X=$ Lesioned cerebral peduncle. $P=$ intact cerebral peduncle.

Graphs showing the probability of the rat crossing at different gap distances in its normal state (i), with a lesion in the right peduncle and both sets of whiskers (ii), with a lesion in the right peduncle and only its right whiskers (iii), and with a lesion in the right peduncle and only its left whiskers (iv). 
Figure 39.

Case VI.

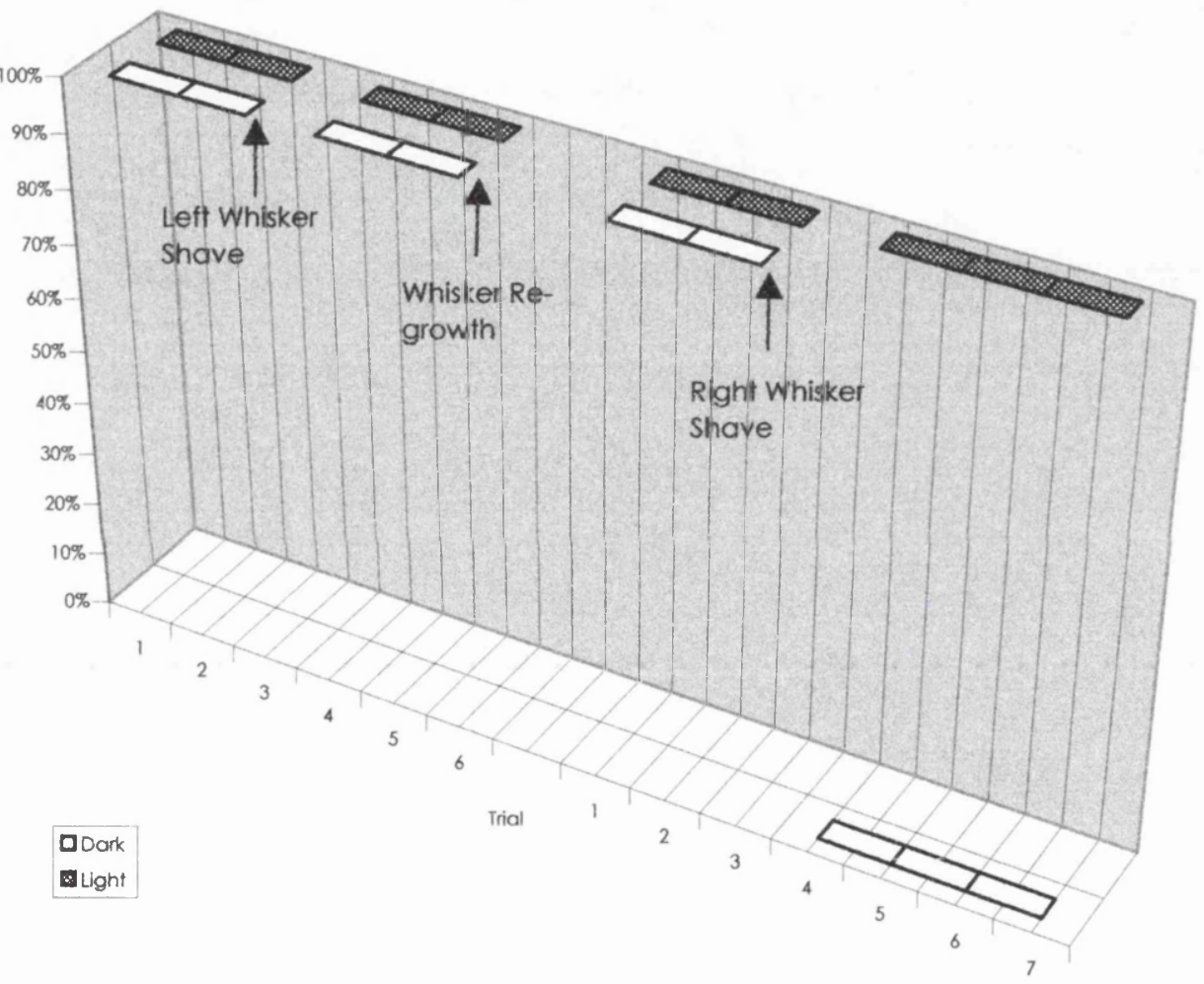

Graph showing the percentage success per trial at a gap of 16 $\mathrm{cm}$ in the dark and light. The rat was first tested as normal, then with a lesion in the right peduncle, followed by cutting all the left whiskers. The whiskers were then allowed to re-grow before the right whiskers were cut. 
Figure 40.

Case VI.

Pontine Nuclei Injection.
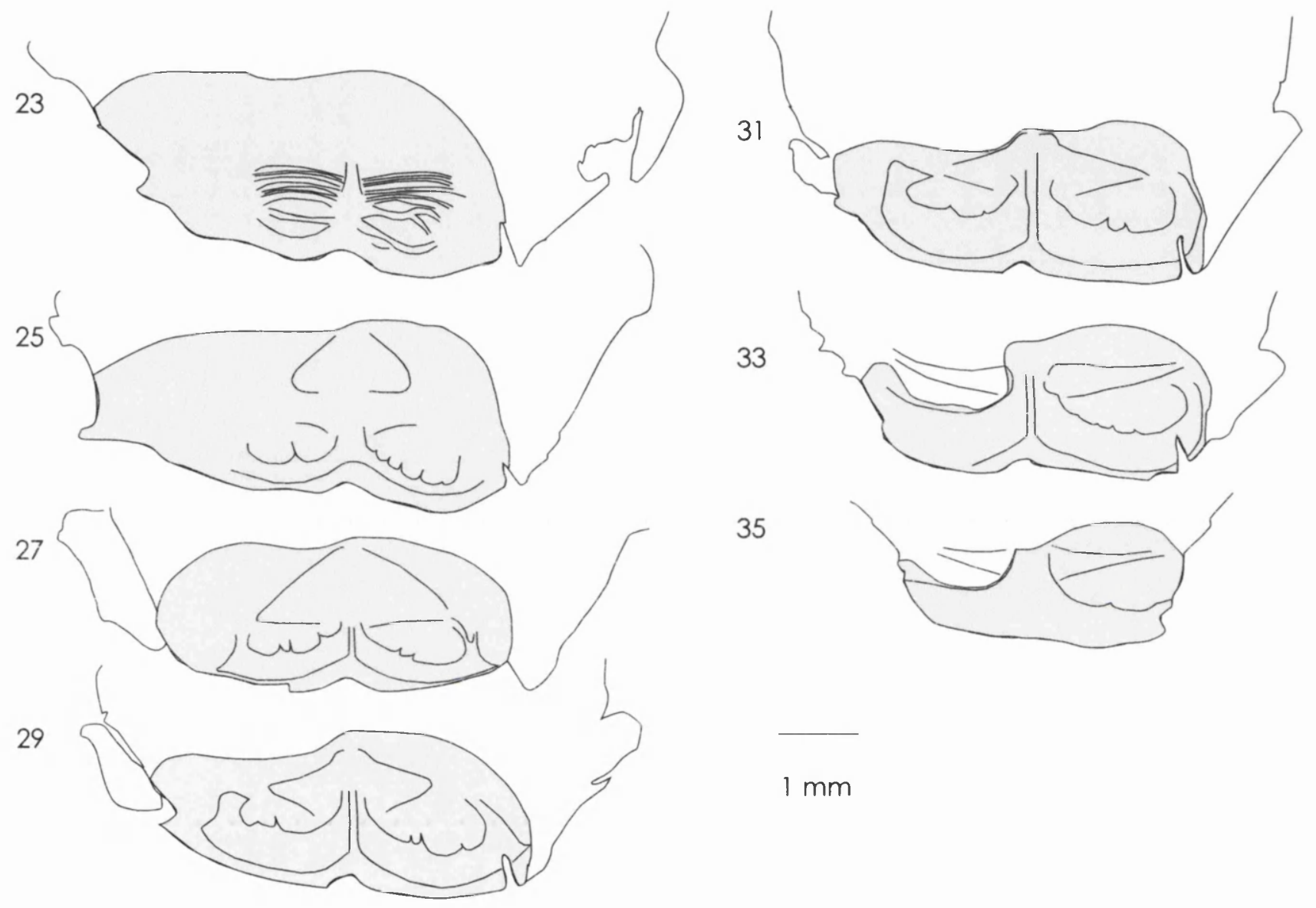

33

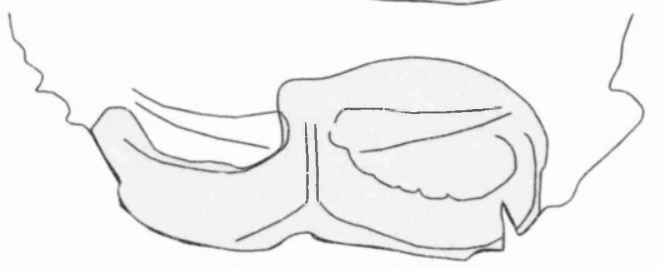

35

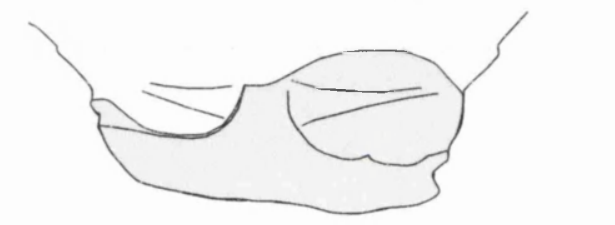

$1 \mathrm{~mm}$

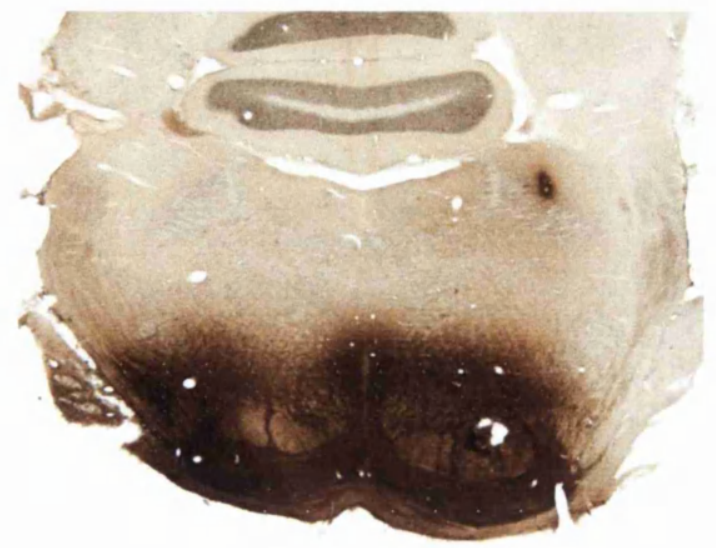

Above:

Extent of injection in pontine nuclei. Sections 300 microns apart.

Left:

Photomicrograph of injection site in $D A B$ reacted section. Section 29. 
Figure 41 .

Case VI.

Section 86.
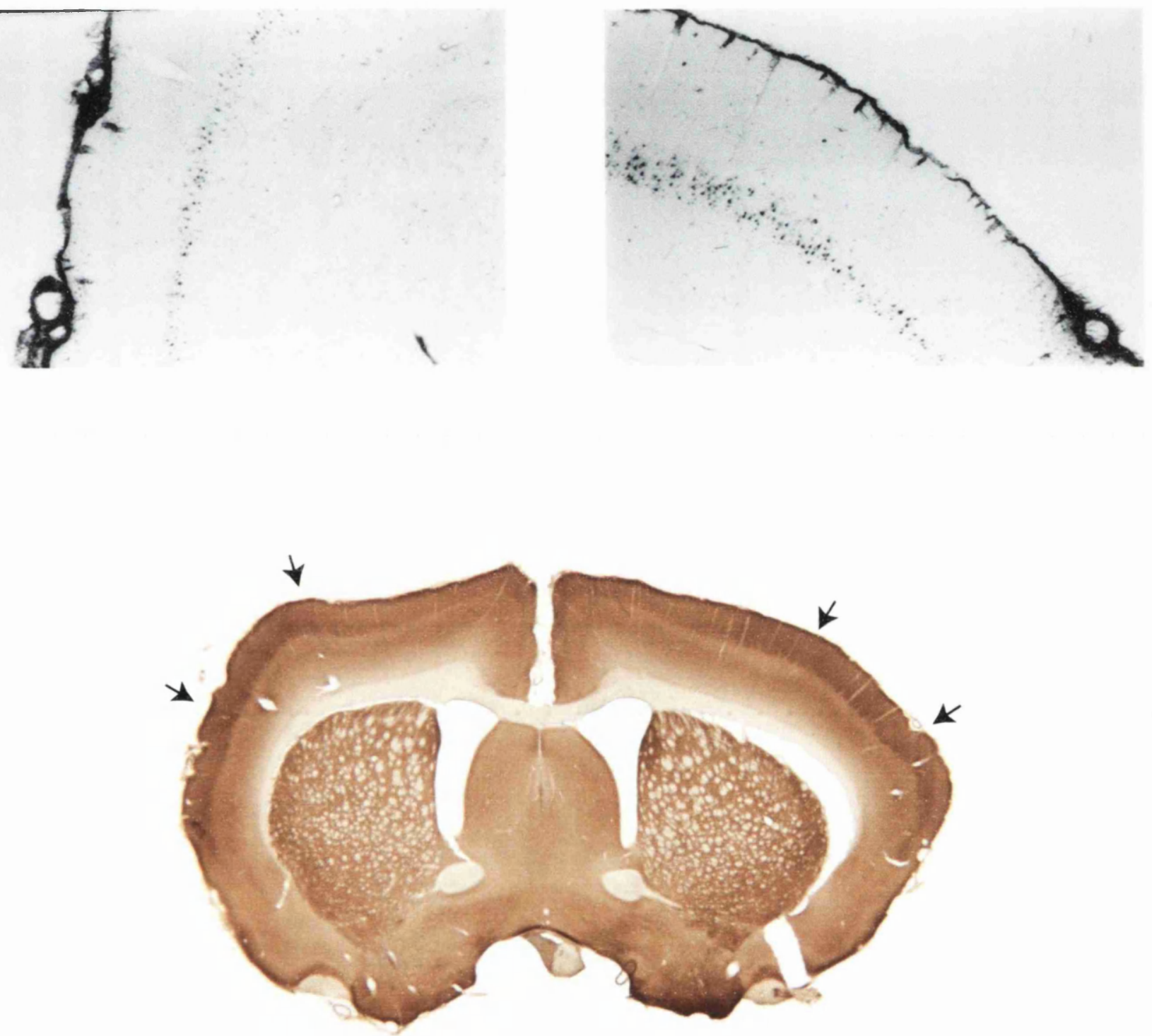

Figures showing the distribution of H.R.P. labelled cells in layer Vb of the cortex and the position of the barrels in layer IV (indicated by arrows). 
Figure 42.

Case VI.

Section 82.
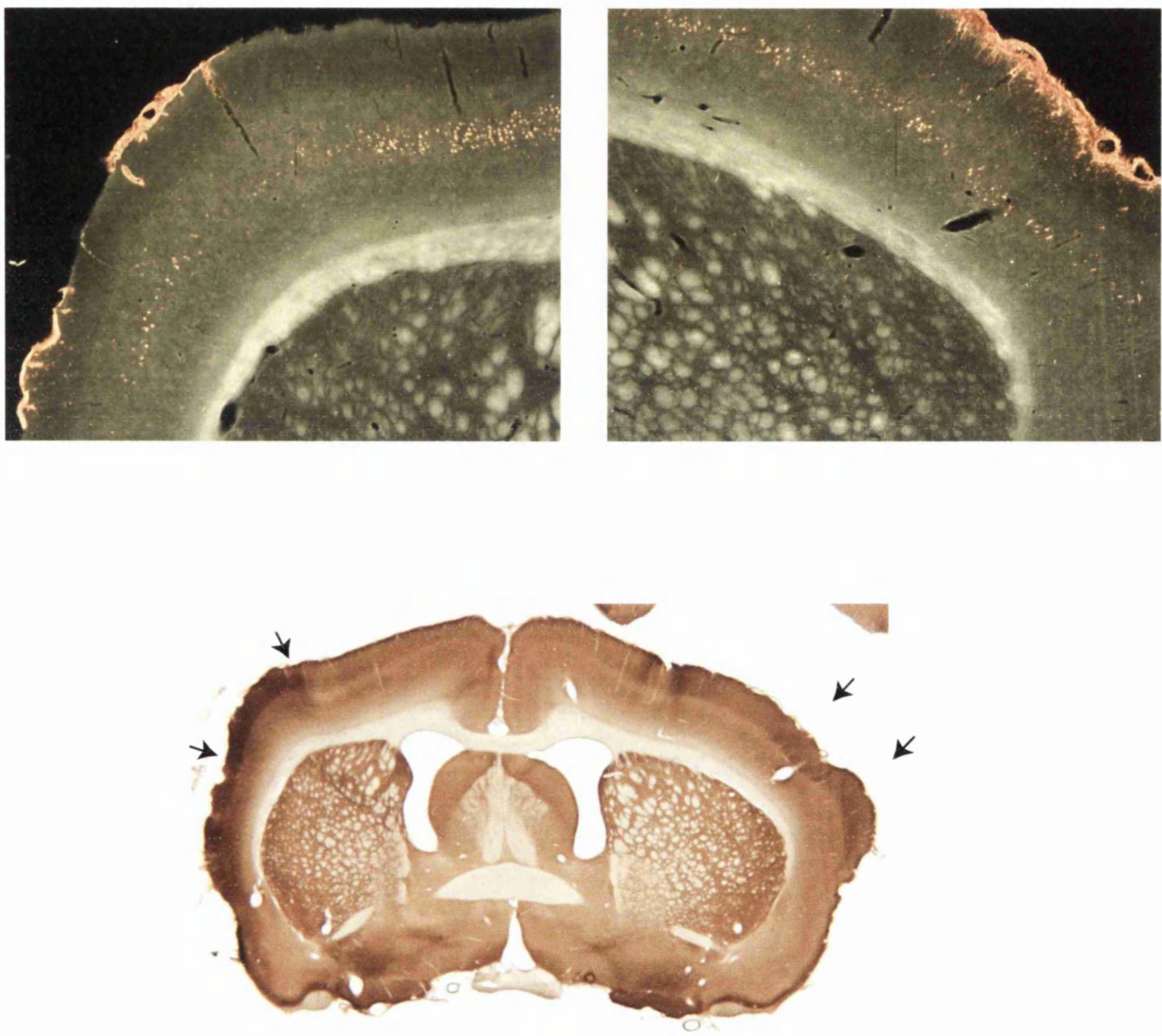

Figures showing the distributon of H.R.P. labelled cells in layer Vb of the cortex and the position of the barrels in layer IV (indicated by arrows). 
Figure 43.

Case VI.

Section 76.
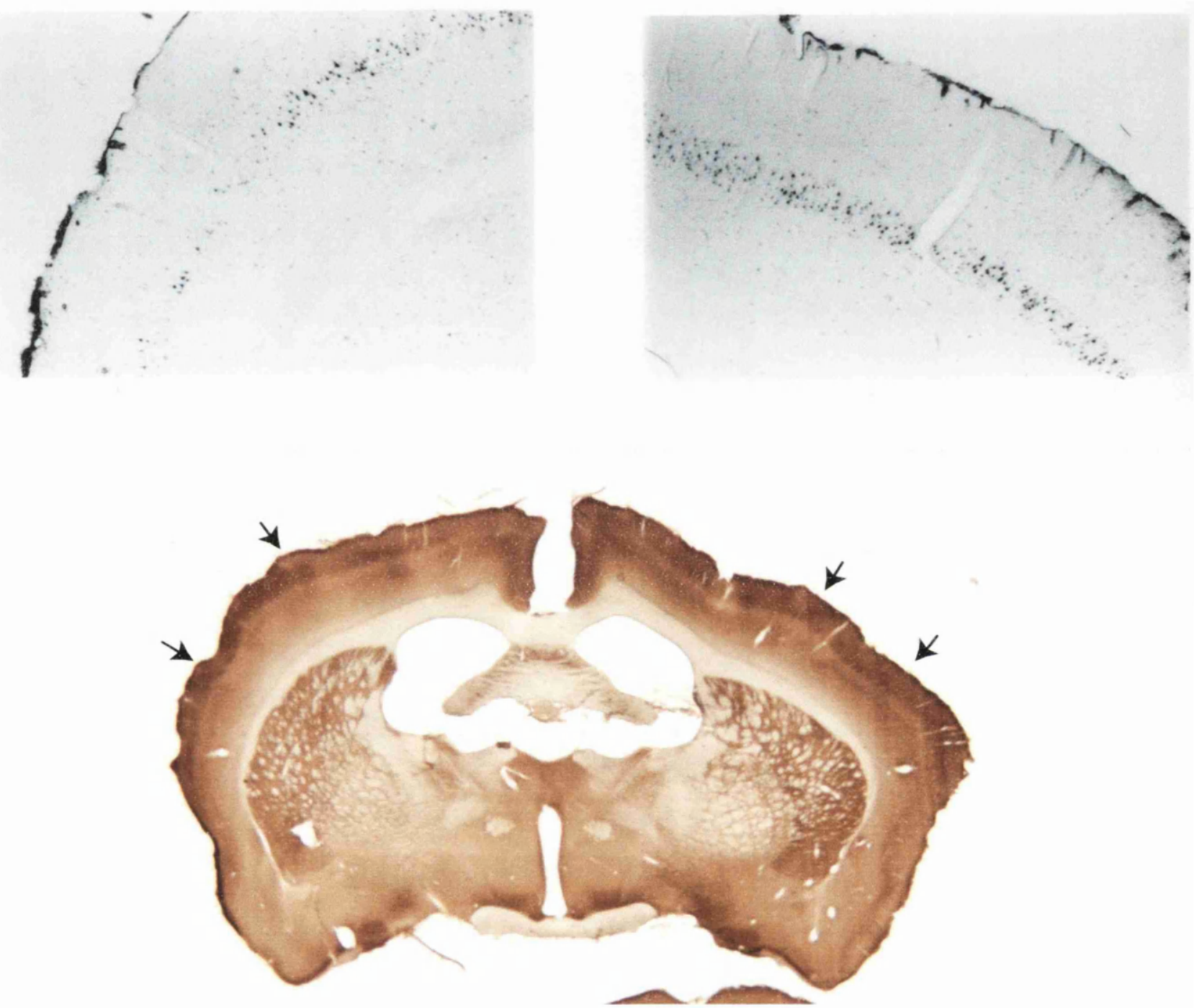

Figures showing the distributon of H.R.P. labelled ceils in layer $\mathrm{Vb}$ of the cortex and the position of the barrels in layer IV (indicated by arrows). 
Figure 44 .

Case VI.

Section 72.
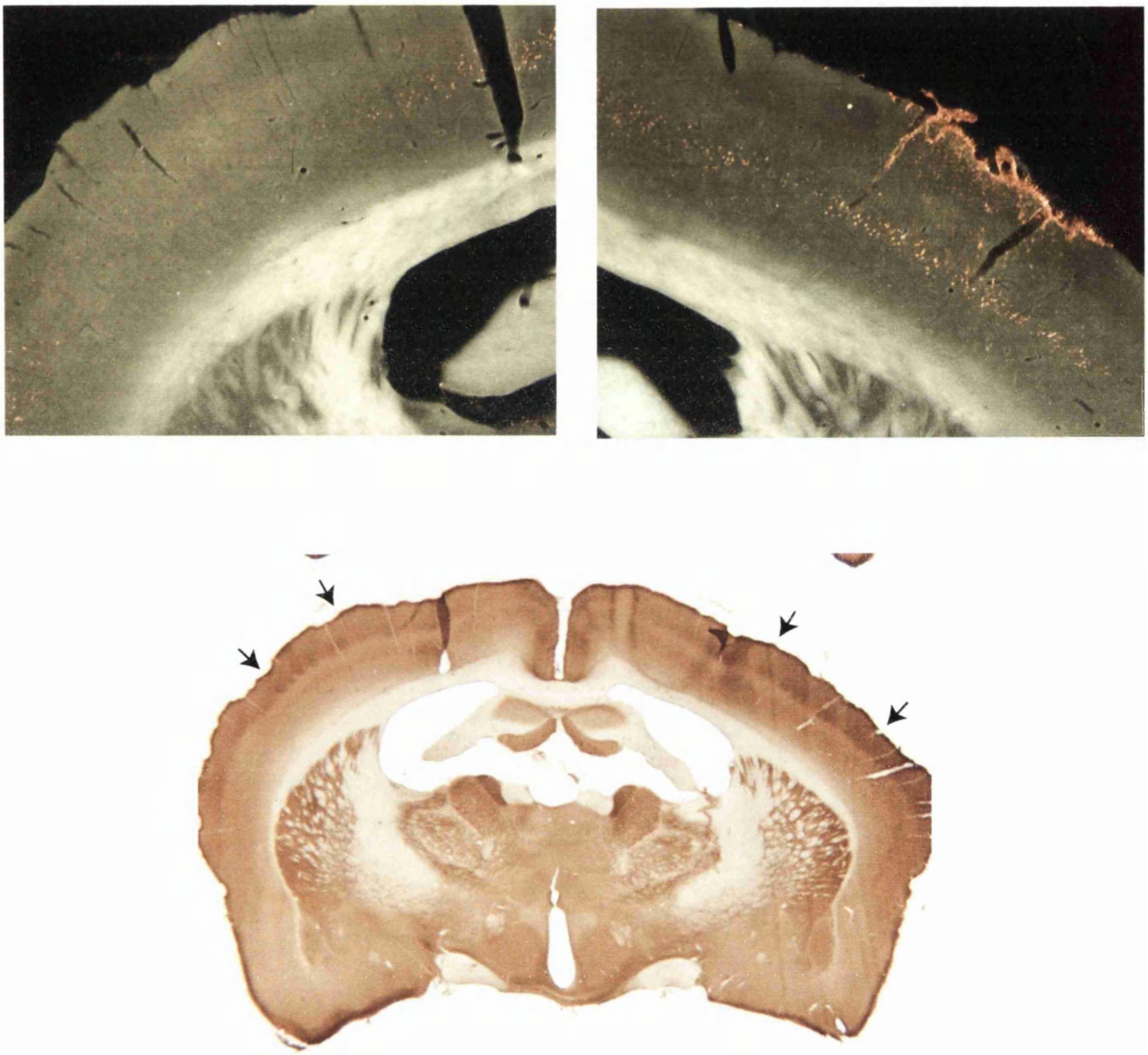

Figures showing the distributon of H.R.P. labelled cells in layer Vb of the cortex and the position of the barrels in layer IV (indicated by arrows). 
Figure 45.

Case VI.

Section 66.
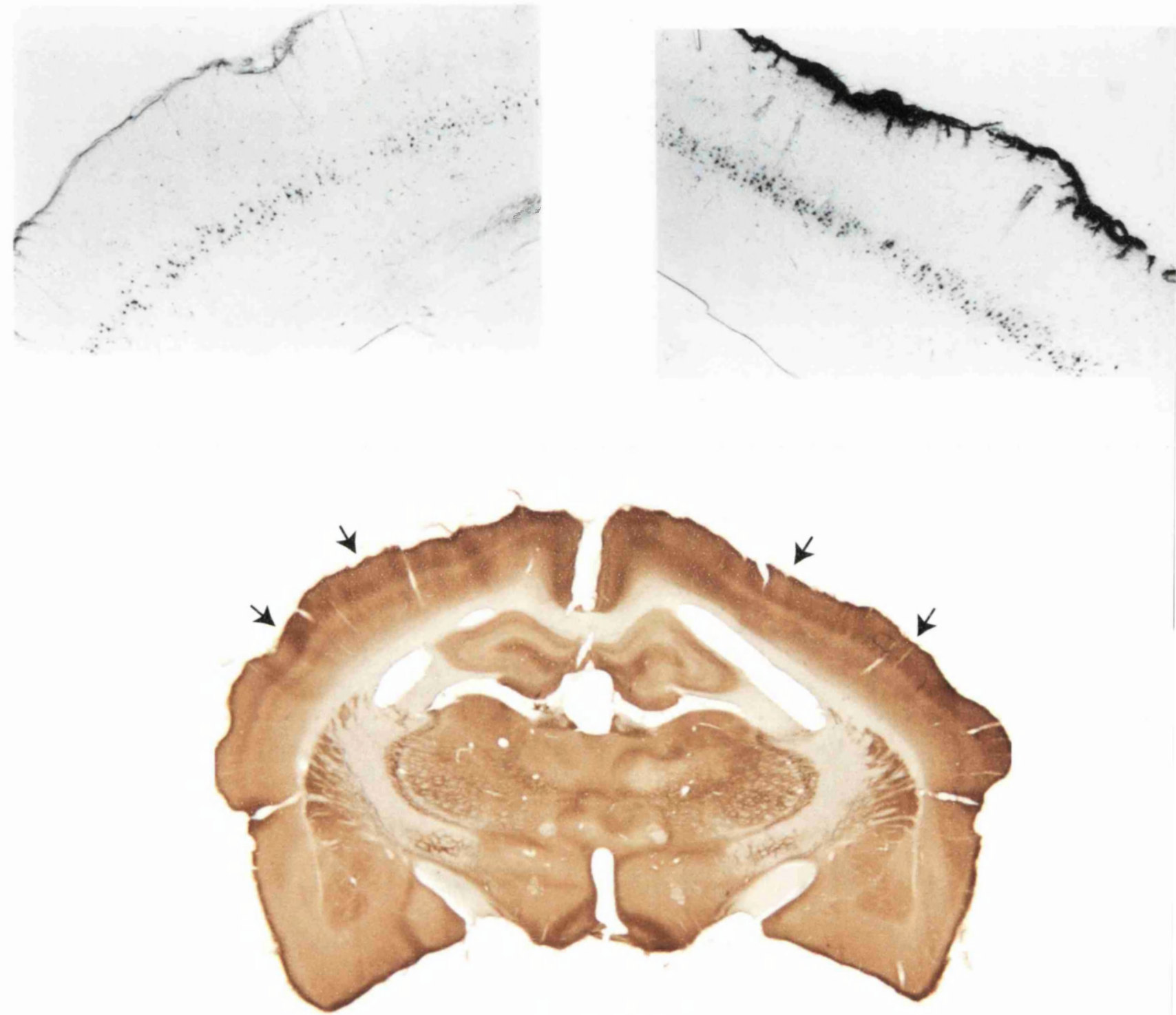

Figures showing the distributon of H.R.P. labelled cells in layer Vb of the cortex and the position of the barrels in layer IV (indicated by arrows). 


\section{Case VII}

Case VII was tested as a normal animal first with a full complement of whiskers. Then with a unilateral whisker shave of all the right whiskers. And finally with a bilateral whisker shave.

Figure 46 (i) and 47 shows that with both sets of whiskers intact the rat crossed consistently at a gap of $16 \mathrm{~cm}$ in the dark. At this distance the animal could only reach across the gap using its whiskers to gauge the distance to jump in the dark.

Figure 46 (ii) and 47 show that following a unilateral whisker shave of the right whiskers the animal continued to cross a maximum distance of $16 \mathrm{~cm}$ in the dark. The animal could be seen to make contact with the far edge of the gap with the remaining set of left whiskers before crossing.

Figure 46 (iii) and 47 show that after the remaining set of whiskers were cut to their bases the animal now refused to cross a gap of $16 \mathrm{~cm}$. After a bilateral whisker shave the animal would now cross a maximum gap distance of $14 \mathrm{~cm}$ in the dark. At this distance the animal used its nose or paw to judge the distance to the far edge before crossing. 
Figure 46.

Case VII.
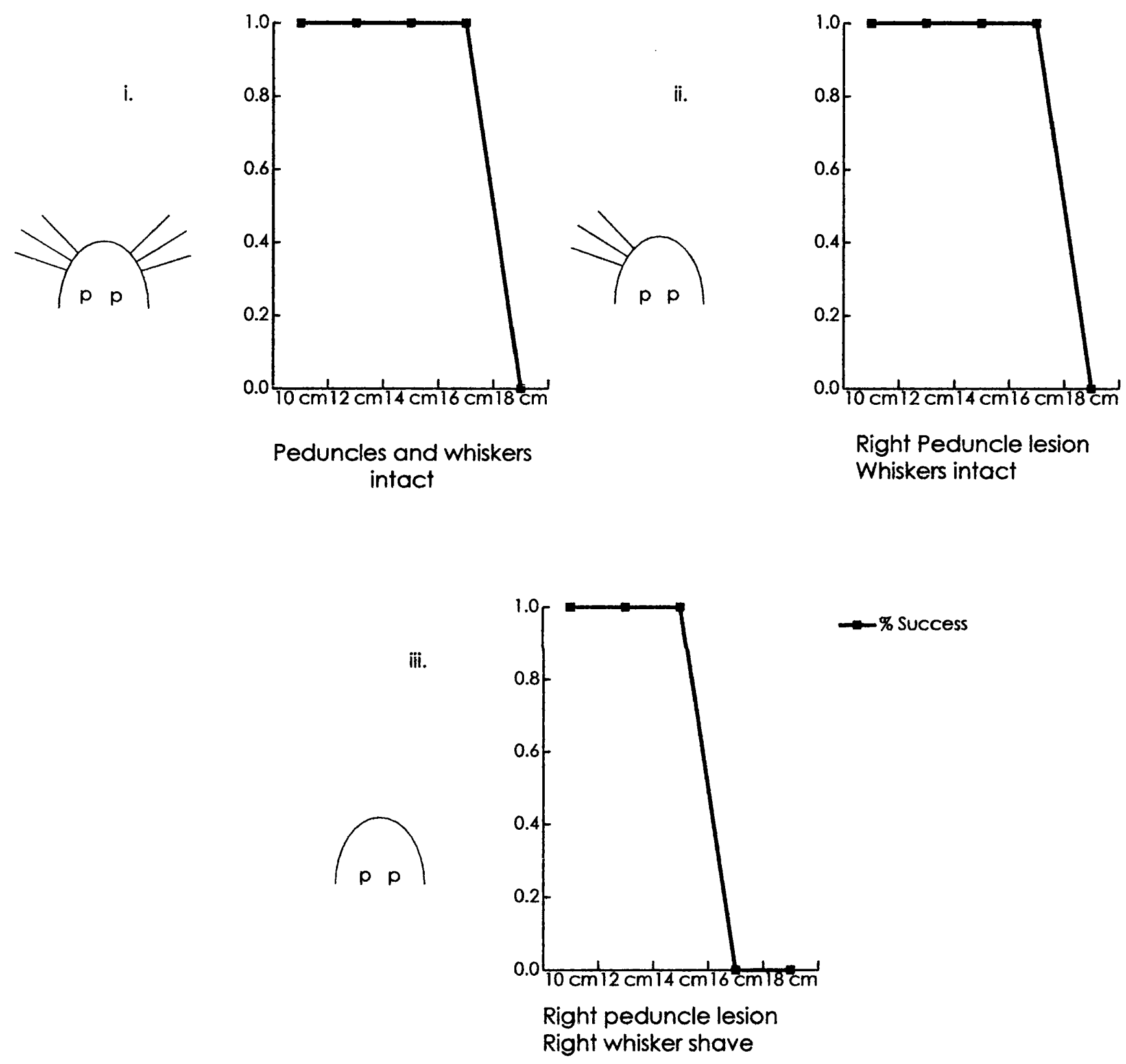

Graphs showing the probability of the rat crossing at differing gap distances in its normal state (i), with a unilateral whisker shave (ii), and with a bilateral whisker shave (iii). 
Figure 47.

Case VII.

\% Success At $16 \mathrm{~cm}$

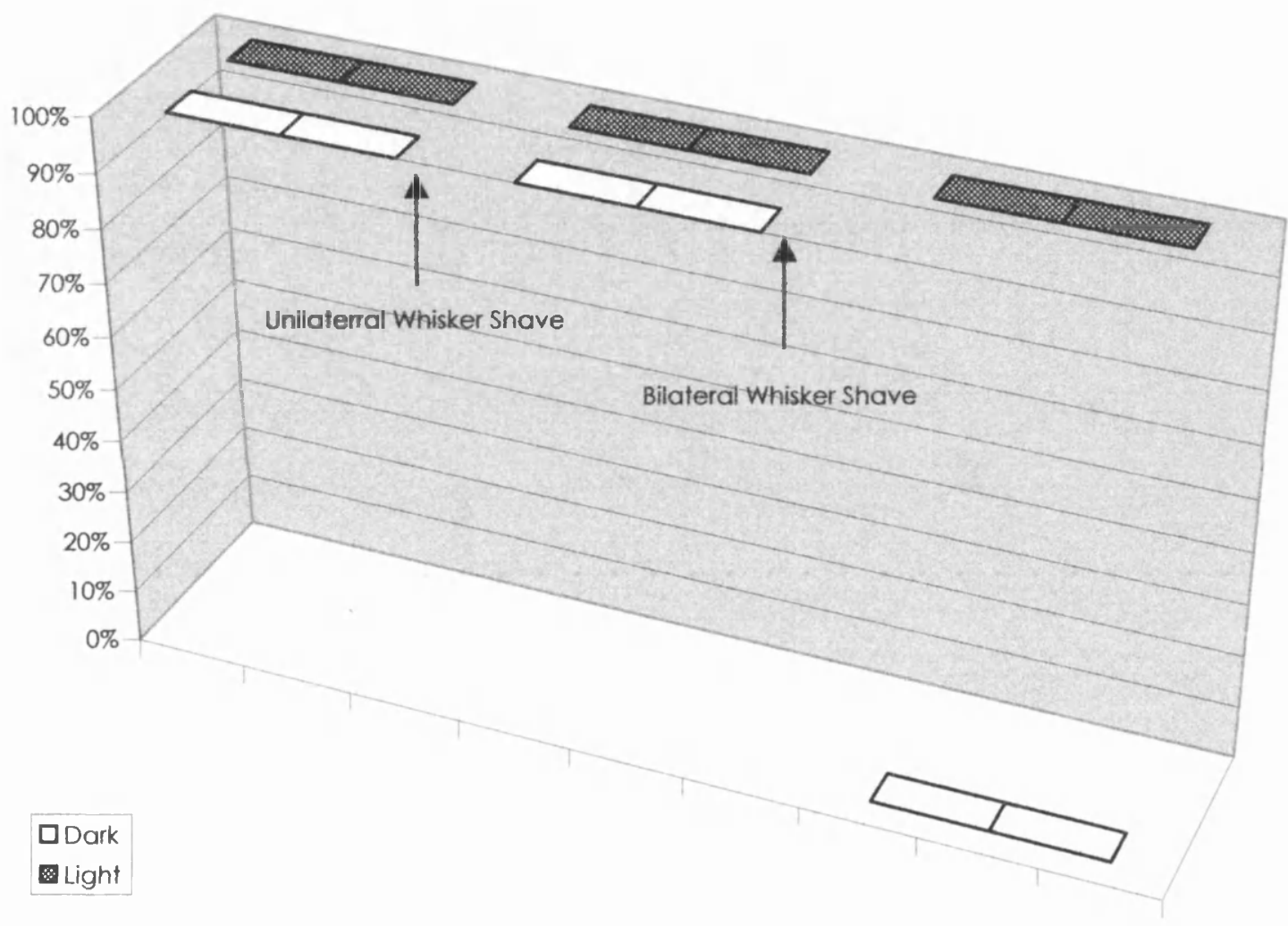

Graph showing the percentage success per trial at a gap of $16 \mathrm{~cm}$ in the light and dark of a normal rat after a unilateral whisker cut, followed by a bilateral whisker cut. 


\section{Case VIII}

Case VIII was tested as a normal rat with a full set of whiskers. The rat then had all the whiskers on both sides of the snout shaved to their bases. The whiskers were then allowed to re-grow to their natural length before the whiskers on the right side of the snout were shaved to their bases.

With a full complement of whiskers the rat was trained to consistently cross a gap of 16 $\mathrm{cm}$ in the dark. Figure 48 (i) and 49 show that with both sets of whiskers cut the animal would not cross a gap of $16 \mathrm{~cm}$. The rat crossed a gap of $14 \mathrm{~cm}$ which he could reach with its nose or paw.

Figure 48 (ii) and 49 show that when the whiskers had re-grown to their normal length the animal could again cross a gap of $16 \mathrm{~cm}$ in the dark. In the dark, at a gap of $16 \mathrm{~cm}$ the animal could only reach the far edge of the gap with its whiskers, and used the whisker to successfully judge the distance to cross.

All the whiskers on the left side of the face were then trimmed to their bases. With only the right set of whiskers remaining the animal behaved as it did with both sets of whiskers (Figure 48, iii and 49). In the dark the animal used the remaining set of whiskers on the right side of the face to reach to the far side of the gap at $16 \mathrm{~cm}$ and correctly judge the required distance to cross. 
Figure 48.

Case VIII.
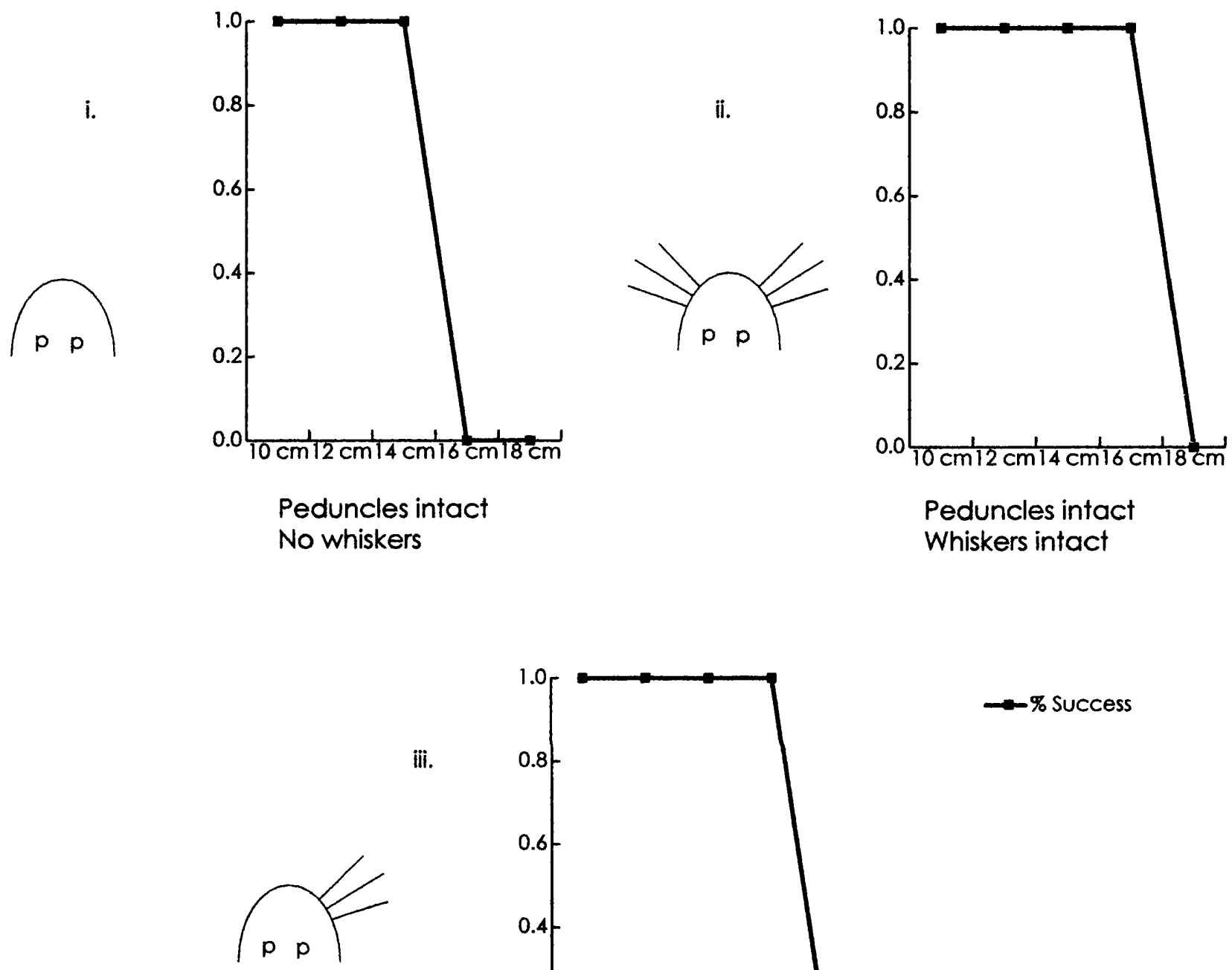

iii.

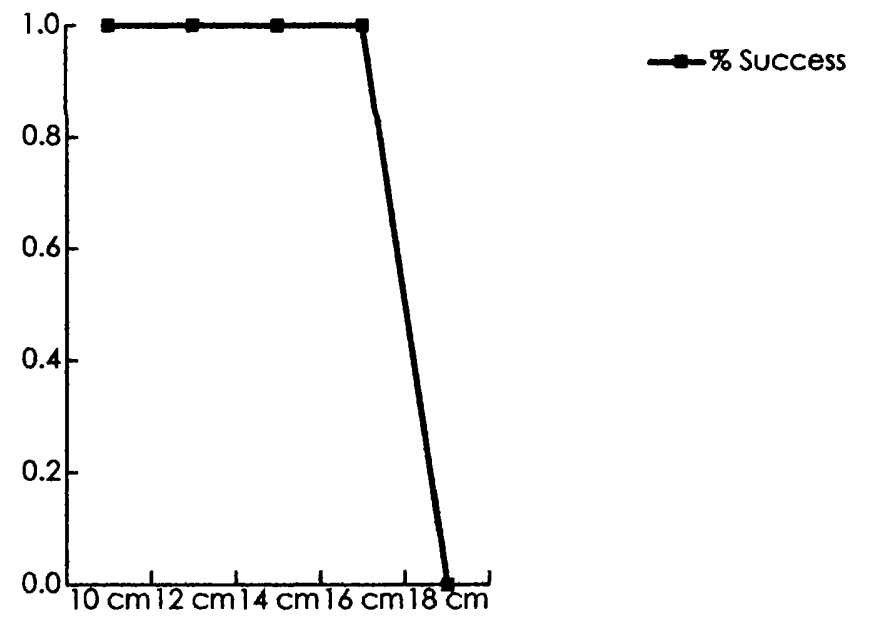

Peduncles intact

Left whisker shave

Graphs showing the probability of a normal rat crossing at differing gap distances with (i) no whiskers, (ii) with whiskers infact and, (iii) only its right whiskers. 
Figure 49.

Case VIII.

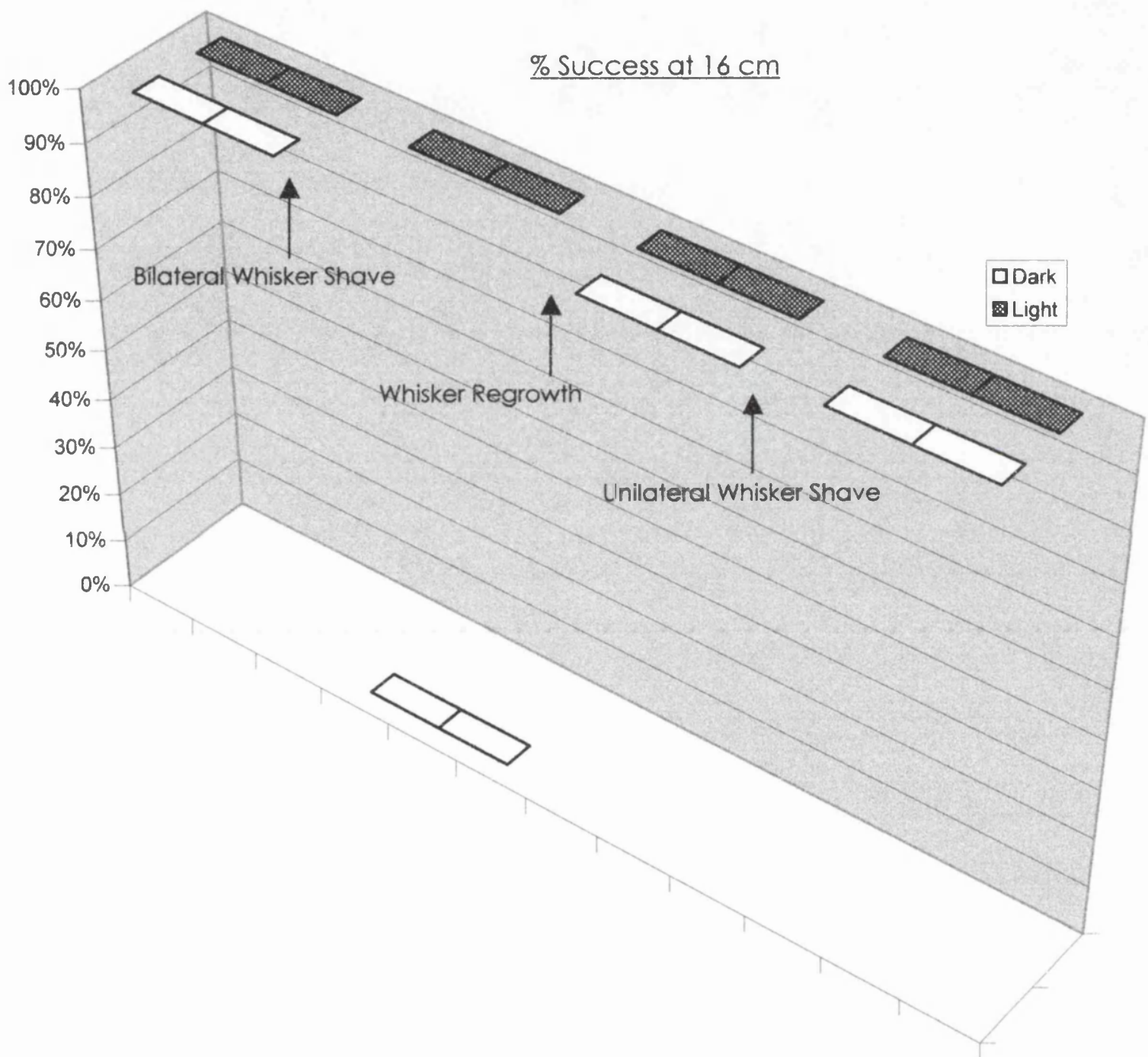

Graph showing the percentage success of Case VIII at a gap width of $16 \mathrm{~cm}$ in the dark and light. The rat was a contral that underwent a bilateral whisker cut. The whiskers were allowed to regrow and then the whiskers were cut unilaterally. 
DISCUSSION 
The introduction reviewed the neuroanatomical pathways to and from the cerebellum, emphasising the dense projections that link sensory areas of the brain to the cerebellum by way of the pontine nuclei. In rats all of the cerebral cortex, including sensory cortex projects to the pons (Legg et al., 1989). In the monkey there is considerable variability in the density of pontine projections from different cortical areas (Glickstein et al., 1985). In the case of vision for example, the pontine projections arise largely from the extrastriate visual areas that are dominated by an input from the magnocellular system and that code for the dynamic properties of a stimulus (Glickstein et al., 1990). These neurones respond at a short latency to visual stimulation. There is a similar projection to the pons from cells that are activated at short latencies from the rat PMBSF, ArmstrongJames et al. (Armstrong-James et al., 1987) found that cells in lamina Vb respond at very short latency to activation of their principle whisker. It is the $\mathrm{Vb}$ cells that relay vibrissal information to the pontine nuclei (Legg et al., 1989; Mercier et al., 1990).

The introduction also reviewed the efferent pathways from the cerebellum, and its powerful connections to structures that connect directly or indirectly to the cells of origin of the descending motor pathways. All these factors are consistent with the idea that the cerebellum receives the type of information that would subserve a system that utilises sensory information to control on-going, fast, accurate movements. Based on these anatomical features, I tested the rôle of the cerebellum in a task that requires the sensory guidance of movement, using whisker-guided movement in rats. In typical lesion studies the function of an area of the brain is tested by destroying a pathway or structure and behaviourally testing the animal. Although this approach has been used for nearly two hundred years to test the function of the cerebellum it does have some potential drawbacks. In the cerebellar cortex a particular region of the body can be 
represented by many small-scattered patches across the cerebellar cortex (Shambes et al., 1978). The divergent "fractured somatotopy" would make it difficult to remove all the areas of cerebellar cortex that receive sensory input from a given sensory area or structure such as the vibrissae. It would be difficult to interpret residual post-lesion performance. The cerebellum might still have remaining sensory input that could compensate for, and allow recovery from the effects of the lesion.

The pontine nuclei receive sensory input from the cerebral cortex and provide the largest source of sensory input to the cerebellum by way of mossy fibres. In principle it might be possible to abolish vibrissal sensory inputs arriving from the cortex by making lesions within the pons. However, like the mossy fibre input to the cerebellum, axons from the PMBSF terminate in several isolated patches throughout the pontine nuclei. Thus making a complete lesion of all the cortical vibrissal terminals in the pons would be difficult.

A direct lesion of the vibrissal representation in the primary somatosensory cortex is a relatively straightforward procedure. Such a lesion would obviously destroy all cortical neurones of the PMBSF that respond to vibrissal stimulation and project to the pontine nuclei. When Hutson and Masterton (1986) performed such a lesion in rats, and tested the animal's ability to guide their movements using only their whiskers, barrel field lesions abolished that ability.

Hutson and Masterton (1986) suggested that although the deficit may have been related to intrinsic properties of the barrel field itself, the impairment in the vibrissal guidance of movement could also have been due to a "sensory-motor disconnection". The PMBSF projects directly to many structures that are involved in the control of 
movement and its ablation would remove the input to all of those target structures. It would be ideal to specifically cut the pathway to the cerebellum whilst leaving others intact, in this way we could attempt to extract the specific rôle of the cerebro-pontocerebellar pathway in a task that requires a rat to guide its movements using only its vibrissae.

A lesion placed in the appropriate part of the cerebral peduncle to cut the descending fibres from the PMBSF would prevent vibrissal information from reaching the pontine nuclei, and therefore the cerebellum. This type of lesion would test the hypothesis based on Hutson and Masterton's (1986) suggestion that by ablating the barrel field they might have caused a "sensory-motor disconnection", and that the critical structure disconnected was the cerebellum.

\section{Experimental Results}

The rats received a lesion that cut the central region of the right cerebral peduncle that contains the fibres originating in the PMBSF of the right primary somatosensory cortex. Since whisker information is virtually entirely crossed at the level of the thalamus, these fibres carry information relating to the whiskers from the left (contralateral) side of the face. Therefore, in all cases the left whiskers barrels were partially or totally disconnected from the pontine nuclei by a lesion of the efferent fibres from the right barrel field. Connections between barrel field cortex and other brain areas, and between other brain areas and the cerebellum remained intact. 
After an appropriate lesion in the right peduncle, cortical sensory information from the left whiskers would not reach the cerebellum via the barrel fields. To test the rôle of this pathway in the animals' ability to use its whisker to judge a gap, we cut the whiskers that retained connections to the pons, sparing the whiskers whose cortical representation no longer projected directly to the pontine nuclei.

The results clearly show that interrupting the barrel field projection to the pons prevents a rat from using the vibrissae whose pontine connections had been severed to gauge the gap. Normal animals with one set of whiskers removed show no deficit, and can still use a single set of whiskers to gauge the distance to be jumped. Animals that had received a lesion to the right peduncle and then had the corresponding (left) whiskers cut, and therefore retained a viable pathway between the (right) vibrissae and the cerebellum via the (left) PMBSF and cerebral peduncle, also could use the remaining whiskers to judge the distance.

The experimental animals received a lesion that interrupted fibres in the peduncle that originated in the vibrissal area of the primary somatosensory cortex. In some of the cases (Case III, Case IV, Case VI) the origin of the cut fibres was verified by mapping retrogradely labelled cells in the PMBSF after large injections of WGA-HRP had been made to fill the entire pontine nuclei. The results were compared with those from an earlier study (Legg et al., 1989) in which the pontine nuclei had been filled with a retrograde tracer

In two of the cases (Case VI \& Case III) the number of retrogradely labelled cells in the cortex overlying the vibrissal representations in Sml was very small, suggesting that virtually all the fibres had been severed. 
In one other case (Case IV), although the number of cells in the PMBSF ipsilateral to the partially cut cerebral peduncle was greatly reduced when compared to the number seen in the cortex contralateral to the lesion, the difference was not as great as in the other two cases. The lesion was, however, well positioned, and large enough to encompass many, if not all of the fibres in the cerebral peduncle that have their origin in the vibrissal barrel field.

In two other cases (Case II \& Case V) the location and extent of the lesion in the peduncle was verified by injection of an orthograde tracer into the vibrissal barrel field on both sides. Since the great majority of cortico-pontine fibres project ipsilaterally, I compared the extent of terminal label in the pons of the two sides with published work on the projections of the sensory facial areas of cortex to the pontine nuclei (Mihailoff et al., 1985), and work in progress in our lab (Hans, personal communication).

In Case II after bilateral injections of BDA into the PMBSF, some labelled terminals were seen on the side of the pontine nuclei ipsilateral to the severed peduncle. The areas of pontine label are in a position consistent with the areas of projection from the face area of the somatosensory cortex (Mihailoff et al., 1985). Far fewer terminals were found in the pontine terminal label on the side ipsilateral to the cut basis pedunculi. The distribution of labelled terminals was reasonably symmetrical across the midline but greatly diminished on the side ipsilateral to the peduncle lesion

Cortico-pontine projections are not strictly ipsilateral. There are patches of terminal label found in the contralateral pontine nuclei after a purely unilateral injection of orthograde tracer into the vibrissal barrel field (Mihailoff et al., 1985). These patches are 
found in the medial areas of the pontine nuclei and could account for the presence of some terminal label in these positions ipsilateral to the damaged peduncle. The other label that is found at more lateral sites is symmetrical to the site of dense label on the contralateral side. This label may reflect the presence of some spared fibres linking the PMBSF to the pontine nuclei.

The injection site in the right and left hemispheres in Case II includes different amounts of the barrel fields. The right injection was almost exclusively contained within the ventral whisker rows $C, D$ and $E$. The injection site in the left hemisphere is larger than that in the right. The spread of $\mathrm{BDA}$ in the left cortex is larger extending to some of the representations of the more rostral and dorsal whiskers. The largest difference however, is in the extent to which the injection site covers the areas of barrels that represent sinus hairs. Areas of cortex that contain the representations of the sinus hairs are known to project bilaterally (Hans, personal communication) and the injection spread in this area probably contribute to the label ipsilateral to cut peduncle. The unevenness of the size of the injection sites could be a contributing factor to the bias toward more labelled fibre terminals in the left of the pontine nuclei. However, the size and position of the lesion within the peduncle and the fact that the label is greatly decreased on the side of the damaged peduncle suggest that the lesion did sever many, if not all of the fibres in the peduncle that connect the PMBSF to the pontine nuclei.

In Case V, WGA-HRP was injected into both vibrissal barrel fields. The results in this case were much easier to interpret. The injection sites were similar on both sides of the brain, centred over the barrels representing the rostral mystacial whiskers. The injection on the right side was slightly larger than that on the left, but both covered the same area of the whisker barrel field. Only the very caudal whiskers of all rows and the straddler 
whiskers were not within the injection sites on either side. The injection in the right hemisphere extends further medially out of the vibrissal barrel field.

There was a large difference in the density of labelled fibre in the longitudinal fasciculus and in the pontine terminal label on the two side of the brain. As the injection site in either hemisphere encompasses a similar amount of the PMBSF, the data suggest that the bias toward the amount of orthograde label in the left side of the brain is due to the disconnection of the pons from the PMBSF by the lesion in the right basis pedunculi. The anatomical position of the peduncle lesion is such that it would have interrupted many of the efferent fibres from the PMBSF.

In Case I there was no verification of the origin of the lesioned fibres using a neuronal tracer. The lesion however, was among the larger lesions in the study. It was large enough and the appropriate position to destroy many if not all of the fibres in the cerebral peduncle that had their origin in the PMBSF of the somatosensory cortex.

The locations of the peduncle lesion in all cases suggest that they would have damaged many or all of the fibres in the cerebral peduncle that originated in the vibrissal barrel field. In all but one case these facts were verified by using anterograde or retrograde tracing techniques. Although some cortico-pontine fibres may have been spared, none of the animals were able to use the set of whiskers that had been disconnected from the pons to judge a gap to cross in the dark.

Interestingly Hutson and Masterton (1986) found that a blind rat could use a single whisker to judge a distance in a similar gap-crossing task as long as the corresponding barrel for that whisker was intact. If the whisker's corresponding barrel was destroyed 
then the animal could no longer perform the task. Thus, we have to ask the question. Why would rats with a single barrel use the corresponding whisker to cross a gap whereas a rat with an incomplete lesion that may have spared some connection between the PMBSF and the cerebellum would not make use of the surviving connections?

Hutson and Masterton's (1986) experimental methodology differed in important respects from ours. The rats in their study were all rendered permanently blind by bilateral enucleation at the outset of the experiment. Blinding the animals would render them absolutely reliant on the use of their whiskers all the time. The whiskers were also trimmed down to a single whisker. It may be that after the loss of vision the animals were primed to become more efficient at using their remaining whisker to navigate in their environment. The animals in our study were not blinded and all could still use vision to judge the distance to jump.

It has been suggested that the different length whiskers at different positions on the face might give the animal a simple way of detecting the minimal distance to an object (Brecht et al., 1997). A possible, simpler explanation is that if some of the projections from the PMBSF to the pons remained intact they may not have belonged to whiskers that are used to detect the distance to the far edge of the gap. The maximum distance the animals crossed in the dark was at the absolute limits of the animals' ability to reach with its vibrissae, only the tips of certain whiskers touching the far edge. It would therefore only require the fibres from a certain subset of whiskers to be interrupted to render the barrels of those whiskers used in the task disconnected from the pons. 


\section{Other Possible Reasons For The Deficit}

It might be argued that the animals' inability to cross the gap post lesion was the result of a general motor deficit. The cerebellum is known to be involved in the regulation of movements and reflexes. Behavioural deficits following cerebellar damage tend to be motor in their nature. Cutting a portion of the cerebral peduncle, which carries fibres from the cortical hemispheres to the pontine nuclei, which in turn is the major mossy fibre input to the cerebellum, could be expected to produce some general disturbances in the normal function of the cerebellum. It would not be unreasonable to expect that any form of damage to the inputs to the cerebellum could disrupt its normal function, which might in turn manifest itself in a deficit in motor performance.

There are several observations that demonstrate that the deficit can not simply be explained as the result of a general degradation of the animal's motor skills. Firstly, after unilateral lesions in the cerebral peduncles the animals were quickly re-trained to the same levels of performance they had displayed prior to the peduncle section. If a general motor deficit were present it is likely to have been at its most severe immediately after the surgery. No motor deficit was revealed after surgery. The animals rapidly achieved pre-operative levels of performance.

Also, in all cases the animals retained the ability to jump large gaps using vision to guide the jump. The size of the gaps varied from 24 to $34 \mathrm{~cm}$, regardless of peduncle lesions and, or whisker cuts. The fact that the animals showed no deterioration in this skill following surgery is additional evidence that there was no general decline in the animals' motor abilities. 
In certain cases (Case I and Case VI) in which whisker guided jumping had been abolished after the peduncle lesion and whisker shaving, training was continued while the whiskers re-grew. In these cases the animal's performance recovered when its whiskers re-grew. Specifically the trimming of the whiskers ipsilateral to the cut peduncle causes the deficit. The animal could not use the whiskers whose corresponding PMBSF had been disconnected from the pontine nucleus.

Another possible reason for the inability of the animal to use the left whiskers to judge a gap is that the lesion in the right peduncle somehow made the whiskers "numb" or caused the animal to ignore the whiskers. However, as Hutson and Masterton (1986) demonstrated, rats with a total PMBSF lesion can still detect passive stimulation of a whisker with the same sensitivity as a normal rat, it would therefore be unlikely that cutting the efferent fibres from the PMBSF in the cerebral peduncle would affect whisker sensitivity.

However, to ensure that the animals could still feel and react to their whiskers before and after the lesion two animals observed (Case $V$ and Case VI) during orientation testing. The animals were touched on their whisker and other parts of the body with forceps holding reward pellets. As described the animals appeared not to change their localisation-orientation behaviour after the lesion. Although the testing was simple and the number of animals small they were able to orientate to tactile stimulation to the whiskers and body surface both before and after surgery to cut the cerebral peduncle. Also, the animals id not show any unilateral bias in their ability to detect passive stimulation of their body. 
In all of the lesions there was some collateral damage to the substantia nigra pars reticularis, which lies immediately dorsal to the cerebral peduncle. Another question we must address is whether the lesion has caused damage to a nearby structure that could have resulted in the rat's inability to use its whiskers to judge the gap to be crossed. The substantia nigra pars reticularis along with the globus pallidus constitutes the main output of the basal ganglia. Damage to one of these structures might cause basal ganglia symptoms. Damage to the basal ganglia is characterised by a paucity of movement. Depending on the structure involved the motor disturbances can be involuntary movement i.e. tremor, disturbance in posture and muscle tone and poverty and slowness of movement, but not paralysis. It would therefore be expected that significant damage to the substantia nigra would produce some general motor deficit.

Some of the animals were seen to turn ipsiversally toward the side of the lesion for a brief period of time after surgery. Circling behaviour following a substantia nigra lesion is well noted in the literature. In the case of our animals the behaviour was transient and was seen only immediately following peduncle surgery. The behaviour was most obviously seen as the animal was recovering from the effects of the anaesthetic, and always completely ceased after twenty-four hours. The circling was not present when the animal was later re-anaesthetised in order to trim the whiskers, nor during recovery from the anaesthetic used during the surgery to inject the pons, or PMBSF later in the experiment. The deficit appears to be transient and completely recoverable.

If the lesion had in fact significantly damaged the substantia nigra pars reticularta, and hence the output of the basal ganglia, we would expect it to affect the animals overall level of motor performance. As has been described the animals' motor performance was unaffected in the immediate training period after surgery to cut the cerebral 
peduncle. The animals did not suffer a general loss of motor function. In all cases the rats successfully jumped large gaps in the light using vision to guide their movements. Jumping these gaps required highly co-ordinated, accurate movements. Any disturbance in global motor control or performance would be expected to impair the ability to perform these visually guided jumps. The small additional damage to the substantia nigra pars reticularis did not affect the animals' performance.

After the unilateral lesion of the cerebral peduncle some of the rats displayed an interesting behavioural phenomenon. When put back into training some of them changed the manner in which they leaned across the gap to whisk the far edge in the dark. Normal animals would lean out straight across the gap palpating the far side of the alley with both sets of whiskers. After the lesion of the right peduncle the animal would reach the gap and lean across diagonally to the left. I suggest that this is a strategy to expose the right whiskers, those that retain their connection between the PMBSF and the pons, to the far edge of the gap. The interpretation is reinforced by the fact that a similar behavioural manifestation is seen in normal animals that have had their left whiskers trimmed to their bases.

\section{Differences Between Vibrissal And Visually Guided Jumping}

Rats with lesions of the visual cortex can still use vision to gauge their jumps (Ellard et al., 1986). The ability of a rat to use visual information after a visual cortical lesion appears at first to be at variance with the data presented in the work of Hutson and Masterton (1986) and this study. 
However, although animals can still use vision to gauge a gap, a lesion of the visual cortex (see Introduction) degrades performance. Although the animal does retain some ability to judge the distance the performance becomes more variable (Ellard et al., 1986). The result is very different from that seen when a rat is required to use its whiskers to gauge a gap after the corresponding vibrissal barrel field has been excised. In such a case the animal's ability is abolished, not degraded (Hutson and Masterton, 1986). The differences in performance are probably related to underlying differences in the anatomical representation of vibrissal and visual information.

In rats $90 \%$ of retinal ganglion cells project directly to the superior colliculus (Dreher et al., 1985; Linden and Perry, 1983). Estimates of the population of retinal ganglion cells that project to the dorsal lateral geniculate nucleus and hence relay information to the visual cortex is in the region of $20-50 \%$ (Dreher et al., 1985; Linden and Perry, 1983; Dreher et al., 1985). In marked contrast the population of neurones in the sensory trigeminal brainstem complex that project to the cortex via the thalamus dwarfs the projections from the same nuclei to the superior colliculus (Bruce et al., 1987). The retino-tectal pathway probably underlies many visually guided behaviours (Dean et al., 1986; Ellard et al., 1986; Goodale et al., 1978; Schneider, 1969; Westby et al., 1990).

In addition to their thalamo-cortical connections, vibrissal areas of the sensory trigeminal brainstem nuclei also project directly to other areas of the brain that have influence on motor output, e.g. the superior colliculus and the cerebellum (Huerta et al., 1983). However, even if these direct pathways are intact the loss of the vibrissal barrel field is tantamount to the loss of the whiskers themselves when the animal has to use whiskers to judge a gap (1986). The cortex is either essential, or an essential link in a pathway 
whose destruction prevents the rat from using its whiskers to guide its movements over a gap. Our study suggests that it is the latter.

An extrageniculate pathway by way of subcortical structures may underpin the retention of the ability to use vision to judge a distance to be jumped after a lesion of the visual cortex. An animal will use many different cues to extract distance information from a visual scene such as motion parallax, retinal disparity cues, and loom. Of these it appears that the extrageniculate system specifically utilises retinal image size to gather distance information, since manipulating this type of cue changes the distances the animal attempts to jump (Carey et al., 1987).

Visual and tactile information may differ in their behavioural importance for guiding movement in different animals. There are many cues that the animal can use to judge a distance and different anatomical pathways underpin the extraction of these different cues.

The use of the whiskers as distance detectors may be more complex than is first apparent. Whisker use is mechanically and behaviourally complicated /Carvell and Simons, 1990; Wineski, 1983). Whisking is an active process; extraction of retinal image size from the environment is not. In some ways whisking is similar to the use of parallax information to extract distance cues. Rats produce motion cues by "head bobbing", in this way vision becomes an active, self produced stimulus that is used to gain distance information from both motion cues (Ellard et al., 1984; Legg and Lambert, 1990). Interestingly it is the use of parallax information for distance cues that is specifically impaired after cortical lesions of visual areas (Ellard et al., 1986). 
All layer Vb pyramidal neurones in the PMBSF project to the pontine nuclei (Legg et al., 1989). Many of these cells have bifurcating axons that also project to the superior colliculus (Mercier et al., 1990). These efferent cortical axons descend in the cerebral peduncle and bifurcate near the position of our lesions, and slightly more rostrally. Therefore, some of the fibres that would normally project from the PMBSF to the superior colliculus would have been interrupted.

It is conceivable that it is this cortico-tectal pathway may be critical to the animals' ability to judge the gap using only its whiskers. The cortical lesions in Hutson and Masterton's (Hutson and Masterton, 1986) study might have resulted in a "sensory-motor disconnection" between the vibrissal barrel field and the superior colliculus. There is however, evidence to suggest otherwise.

Cells of the sensory trigeminal brainstem complex that project to the thalamus and the superior colliculus form two largely segregated populations of neurones (Chiaia et al., 1991a). The majority of VPm neurones, the main thalamic relay to the barrels, have receptive fields that respond best to a single-central whisker, and less vigorously to the immediately surrounding whiskers. They also display slowly adapting response characteristics that are similar to those seen in the primary somatosensory cortex (lto, 1988; Simons and Carvell, 1989). The response properties of VPm neurones mirror those found in the cells of their main trigeminal input, the principle sensory nucleus.

Cells in the superior colliculus that are activated by the vibrissae, typically respond to several whiskers, with fast-adapting response characteristics that are poorly suited to encode for the direction, displacement and velocity attributes of a stimulus (McHaffie and Stein, 1981). Receptive fields of the cells in the superior colliculus reflect the 
response properties found in neurones in the nuclei that provide its main input, the interpolaris subdivision of the spinal trigeminal nucleus.

A small population of neurones send bifurcating fibres to both VPM and the superior colliculus, these neurones are few in number and tend to be located in interpolaris subnucleus of the spinal trigeminal nucleus (Bruce et al., 1987). Physiological studies reflect these findings. In normal rats there are few vibrissa responsive VPm thalamic cells which respond to whisker stimulation in a manner similar to cells found in the deep layers of the superior colliculus (Armstrong-James et al., 1991b; McHaffie and Stein, 1981). Collicular like response properties are more apparent in VPm following kainic acid lesions to the principle sensory nucleus (Rhoades ef al., 1987). Thus although VPm does receive a small input from cells that project to, and have colliculus like response properties, these are not the inputs that normally provide vibrissal information to cells in VPm and hence the barrel field.

So, it is clear that the superior colliculus and the somatosensory cortex receive different types of information from the periphery from largely separate populations of secondary sensory neurones. The response properties of those cells that project to, and are found in the superior colliculus code for less complicated stimulus qualities and have less refined receptive fields as those that project to the vibrissal barrel field via the thalamus.

The rôle of the descending cortico-tectal projections is unclear. In the case of the visual system of the hamster, if visual cortex is ablated fewer directionally selective neurones are found in the upper layers of the superior colliculus (Chalupa and Rhoades, 1977). In the normal rat, there are very few directionally selectively cells in the colliculus so the function of this projection is less clear. 
The rôle of the somatosensory cortico-tectal projection is even less obvious. If the primary somatosensory cortex of a hamster is removed, the cells of the superior colliculus that respond to the same somatosensory stimuli show no significant changes in their response properties (Rhoades, 1981). Cells of the superior colliculus are driven principally by ascending projections from the periphery. Hamsters that have undergone subtotal lesions of the somatosensory cortex do not display any of the orientation or postural deficits that are associated with lesions of the superior colliculus (Barth and Schallert, 1987; Schneider, 1967).

Although the superior colliculus is a common pathway to orientation systems controlled by both central and peripheral mechanisms, Schneider (1967) pointed out that after superficial superior colliculus lesions, hamsters appeared blind to food that was present in their visual field until the animal made contact with its whiskers. But they had no trouble in localising the food after ablation of the visual cortex. The only time the animals revealed a deficiency was when required to follow the food which was moved rapidly from side to side. In other words when the task was a more challenging test of visually guided movement than a simple orientation localisation task.

The rats' deficit in the present experiments matches that found by Hutson and Masterton (1986). Yet, unlike their experiments, in our study there was no damage to the cerebral hemispheres that contain the barrel representations. Therefore we can say that it is not simply the integrity of the vibrissal representations in the PMBSF that are required for the animals to be able to actively palpate across a gap to ascertain the distance required to cross. 
The cerebellum appears to be crucial to the animals' ability in this task. Thus, both cerebellum and the barrel field appear to be necessary (Hutson and Masterton, 1986). The cortico-ponto-cerebellar pathway underpins the system that underlies the fast, ongoing, sensory guidance of movement required for this behavioural task. If this pathway is interrupted, by ablating the cortex, the system fails. If the efferent fibres in the cerebral peduncle are cut the system also fails.

How general are the effects of peduncle lesion? In humans there are only isolated bits of information that might be relevant. For example, Classen and his colleagues (Classen et al., 1995) demonstrated a similar inability of a patient to use sensory information to guide the motor systems following the interruption of the cortico-pontocerebellar pathway. In their case the descending cortico-pontine fibres from the extrastriate visual areas were interrupted by a subcortical infarct, which destroyed part of the internal capsule. The lesion caused a deficit in the patient's ability to use visual information to guide motor output.

These results suggest that the simple idea that damage to parietal lobe visual areas cause visuomotor apraxia due to severing of cortico-cortical links may need to be reconsidered (see Glickstein et al., 1998). In fact, the ablation of the parietal areas that contribute heavily to the cortico-ponto-cerebellar pathway may be performing "sensory motor disconnection" of the visual system, analogous to the deficit in gap crossing.

One test of the rôle of sensory input to the pons would be to compare the performance of animals in which all of the fibres of the cerebral peduncle had been cut with those in which only the cortico-spinal fibres were damaged. 
In a study to investigate the effect of severing the pyramidal tract on movement of monkeys Bucy and his colleagues (1966) attempted to completely sever the cerebral peduncles. Even animals that had almost total or complete degeneration of the pyramids would recover some skilled use of their limbs and hands. After the animals' recovery had stabilised they were tested on several specific motor tasks. One of the tests had the animals required to perform was to pick food off a turntable. The test was designed to test the animals' ability to guide the hand accurately. The ability of the animal to perform this task was indicated by the maximum speed at which the animal was successful at taking pellets from the turntable. Animals with lesions that cut both the cerebral peduncles almost totally or completely were massively impaired at this task.

One animal with a bilateral ablation of the cerebral peduncle showed a much smaller post-surgical deficit performing this task. This animal underwent serial peduncle cuts. The first peduncle cut almost completely destroyed the pyramidal tract, after which the animal could use the unaffected hand to take food as well as a normal animal. The second cut only destroyed the ventral part of the middle half of the peduncle, the part of the peduncle that contains the cortico-spinal fibres. Following the second surgery the animal could use both arms equally well and much better than the preferred arm of any of the animals that had received more extensive damage to both cerebral peduncles.

The lesions in each peduncle had caused different amounts of damage to the motor cortex output to the spinal cord. If each motor cortex were the structure that predominantly controlled the contralateral arm, the deficit in each arm's performance 
would reflect the amount of damage to the cortico-spinal tract in the peduncle. In fact the two limbs performed equally despite the fact that the lateral two thirds of one peduncle were completely lesioned and the pyramids on this side were totally degenerated, whilst in the other peduncle the lesion destroyed the ventral half of the cortico-spinal fibres.

This animal that retained much of its performance in both limbs had a second lesion that preserved all of the descending fibres from the parietal cortex. Many of these fibres are connected to the pontine nucleus and cerebellum in turn, and carry visual information about the dynamic nature of stimuli. The cerebellum has bilateral access to descending motor pathways other than the cortico-spinal pathway that control limb movement. It may be that it is this pathway that underlies the residual performance of the animal.

In conclusion, there is direct evidence presented in this thesis that sensory input used for the sensory guidance of movement is processed in the cortex and is connected through the cerebellum to motor elements of the brain. The evidence suggests that if this cortico-ponto-cerebellar pathway is interrupted at either the level of the cortex (Hutson and Masterton, 1986) or at the level of the descending sensory cortical fibres to cerebellum an animal can no longer guide its movements using sensory information.

The direct evidence of this thesis is supported by clinical evidence in humans /Classen et al., 1995) and by re-interpretation of other results (Bucy et al., 1966; Hutson and Masterton, 1986)and suggests that the cerebellum is a major route through which sensory information is processed to produce relevant motor action. 
APPENDIX I 


\section{$\underline{\text { Surgical Protocol }}$}

Individual Peduncle Lesion Co-ordinates

\begin{tabular}{|l|l|l|l|l|l|}
\hline & AP & $\mathrm{ML}$ & Depth & Temp. & Duration \\
\hline Case I & $+3.3 \mathrm{~mm}$ & $-2.8 \mathrm{~mm}$ & $-7.1 \mathrm{~mm}$ & $85^{\circ} \mathrm{C}$ & $4 \frac{1}{2} \mathrm{~min}$ \\
\hline Case II & $+3.3 \mathrm{~mm}$ & $-2.8 \mathrm{~mm}$ & $-7.4 \mathrm{~mm}$ & $95^{\circ} \mathrm{C}$ & $10 \mathrm{~min}$ \\
\hline Case III & $+3.3 \mathrm{~mm}$ & $-2.8 \mathrm{~mm}$ & $-7.3 \mathrm{~mm}$ & $90^{\circ} \mathrm{C}$ & $5 \mathrm{~min}$ \\
\hline Case IV & $+3.3 \mathrm{~mm}$ & $-2.8 \mathrm{~mm}$ & $-7.3 \mathrm{~mm}$ & $90^{\circ} \mathrm{C}$ & $5 \mathrm{~min}$ \\
\hline Case V & $+3.3 \mathrm{~mm}$ & $-2.8 \mathrm{~mm}$ & $-7.3 \mathrm{~mm}$ & $90^{\circ} \mathrm{C}$ & $8 \mathrm{~min}$ \\
\hline Case $\mathrm{VI}$ & $+3.3 \mathrm{~mm}$ & $-2.8 \mathrm{~mm}$ & $-7.3 \mathrm{~mm}$ & $90^{\circ} \mathrm{C}$ & $5 \mathrm{~min}$ \\
\hline
\end{tabular}

Individual Pons Injection Co-ordinates

All animals received eight injections. All co-ordinates were calculated from ear bar zero. These injections were at the following co-ordinates unless otherwise stated. A $4 \%$ solution of WGA-HRP was used in all cases.

\begin{tabular}{|l|l|l|l|l|}
\hline AP & $+0.5 \mathrm{~mm}$ & $+0.5 \mathrm{~mm}$ & $+0.5 \mathrm{~mm}$ & $+0.5 \mathrm{~mm}$. \\
ML & $-1.8 \mathrm{~mm}$ & $-1.0 \mathrm{~mm}$ & $+1.0 \mathrm{~mm}$ & $+1.8 \mathrm{~mm}$ \\
DV & $-0.3 \mathrm{~mm}$ & $-0.3 \mathrm{~mm}$ & $-0.3 \mathrm{~mm}$ & $-0.3 \mathrm{~mm}$ \\
\hline AP & $+1.5 \mathrm{~mm}$ & $+1.5 \mathrm{~mm}$ & $+1.5 \mathrm{~mm}$ & $+1.5 \mathrm{~mm}$ \\
ML & $-1.8 \mathrm{~mm}$ & $-1.0 \mathrm{~mm}$ & $+1.0 \mathrm{~mm}$ & $+1.8 \mathrm{~mm}$ \\
DV & $-0.3 \mathrm{~mm}$ & $-0.3 \mathrm{~mm}$ & $-0.3 \mathrm{~mm}$ & $-0.3 \mathrm{~mm}$ \\
\hline
\end{tabular}


Notes

Case III: All injections as above, $50 \mathrm{nl}$ of WGA-HRP at each co-ordinate.

Case IV: All injections as above, $50 \mathrm{nl}$ of WGA-HRP at each co-ordinate.

Case VI: All injections as above, $50 \mathrm{nl}$ of WGA-HRP at each co-ordinate.

Individual PMBSF Injection Co-ordinates

Case Il: $50 \mathrm{nl}$ of $10 \%$ BDA was injected bilaterally $1 \mathrm{~mm}$ under the cortical surface at an angle of $40^{\circ}$ from the midline vertical. The co-ordinates were $2.5 \mathrm{~mm}$ posterior to bregma and $5.4 \mathrm{~mm}$ from the midline bone suture.

Case V: $30 \mathrm{nl}$ of $4 \%$ WGA-HRP was injected bilaterally $1 \mathrm{~mm}$ under the cortical surface at an angle of $40^{\circ}$ from the midline vertical. The co-ordinates were $2.5 \mathrm{~mm}$ posterior to bregma and $5.6 \mathrm{~mm}$ from the midline bone suture. 


\section{$\underline{\text { Histological Protocol }}$}

\section{Horse-Radish Peroxidase Histology}

\section{Fixation}

The animal is perfused with $1000 \mathrm{ml}$ of normal saline with 1000 units of heparin added, at room temperature, followed by $1000 \mathrm{ml}$ of $4 \%$ paraformaldehyde solution at $4^{\circ} \mathrm{C}$, both pumped at a rate of $40 \mathrm{ml}$ per minute. The fix is flush from the brain with $500 \mathrm{ml}$ of $10 \%$ buffered sucrose solution followed by a $20 \%$ buffered sucrose solution both at $4^{\circ} \mathrm{C}$, pumped at a rate of $60 \mathrm{ml}$ per minute. These final sucrose solutions also cryoprotect the brain.

Normal Saline: $0.9 \%$ sodium chloride ( $\mathrm{NaCl}$ ) solution: $9 \mathrm{~g} \mathrm{NaCl}$ dissolved in $1000 \mathrm{ml}$ of distilled water $\left(\right.$ d. $\left.\mathrm{H}_{2} \mathrm{O}\right)$.

$4 \%$ Paraformaldehyde: $40 \mathrm{~g}$ of paraformaldehyde dissolved in $100 \mathrm{ml}$ of d. $\mathrm{H}_{2} \mathrm{O}$ at $70^{\circ}$ C. Add drops of $1 \mathrm{~N}$ sodium hydroxide $(\mathrm{NaOH})$ until solution clears. Cool to below $40^{\circ}$ C, add distilled water to $500 \mathrm{ml}$, and filter. Add $500 \mathrm{ml}$ of $0.2 \mathrm{M}$ phosphate buffer, and check the $\mathrm{pH}$ is between $7.2-7.6$.

$10 \%$ Buffered Sucrose Solutions: Dissolve $50 \mathrm{~g}$ of sucrose in $500 \mathrm{ml}$ of $0.1 \mathrm{M}$ phosphate buffer solution.

$20 \%$ Buffered Sucrose Solutions: Dissolve $100 \mathrm{~g}$ of sucrose in $500 \mathrm{ml}$ of $0.1 \mathrm{M}$ phosphate buffer solution. 


\subsection{Phosphate Buffer Solution:}

Make up two separate solutions:

A) Dissolve $27.6 \mathrm{~g}$ sodium dihydrogen orthosulphate in $1000 \mathrm{ml} \mathrm{d} . \mathrm{H}_{2} \mathrm{O}$.

B) Dissolve $113.6 \mathrm{~g}$ di-hydrogen hydrogen orthosulphate in $4000 \mathrm{ml}$ of d. $\mathrm{H}_{2} \mathrm{O}$.

Mix solutions $\mathrm{A}$ and $\mathrm{B}$.

0.1 M Phosphate Buffer Solution: Mix equal volumes of $0.2 \mathrm{M}$ phosphate buffer solution and d. $\mathrm{H}_{2} \mathrm{O}$.

\section{Section Processing}

\section{Tetramethylbenzidine (TMB) Chromogen}

Process the sections through the following solutions in mesh bottomed bins:
A) Six changes of d. $\mathrm{H}_{2} \mathrm{O}$ at $4^{\circ} \mathrm{C}$, for 15 seconds each.
B) Pre-incubation solution -40 minutes at $4^{\circ} \mathrm{C}$.
C) Incubation solution -10 minutes at $4^{\circ} \mathrm{C}$.
D) Move reaction to orbital shaker, change incubation solution after each 10 minutes a total of three times.
E) Rinse six times in rinsing solution, 5 minutes each at $4^{\circ} \mathrm{C}$ with agitation.
F) Mount sections onto subbed slides from fresh rinsing solution and left to air dry before dehydration.

\section{$\underline{\text { Required Solutions }}$}




\section{Pre-incubation Solution}

I used a total of $1600 \mathrm{ml}$ of solution for each reaction:

A) $1440 \mathrm{mg}$ of Sodium Ferracyanate and $80 \mathrm{ml}$ of acetate buffer dissolved in $1480 \mathrm{ml}$ of d. $\mathrm{H}_{2} \mathrm{O}$.

B) $80 \mathrm{mg} \mathrm{TMB}$ dissolved in $80 \mathrm{ml}$ of absolute alcohol with ultrasound.

The solution was used in batches of $400 \mathrm{ml}$ made up just before use at a ratio of 39:1 solution A : solution B.

$\underline{\text { Incubation Solution }}$

As pre-incubation solution but activated by adding $1 \mathrm{ml}$ of $0.3 \%$ hydrogen peroxide $\left(\mathrm{H}_{2}\right.$ $\mathrm{O}_{2}$ ) solution $100 \mathrm{ml}$ of solution.

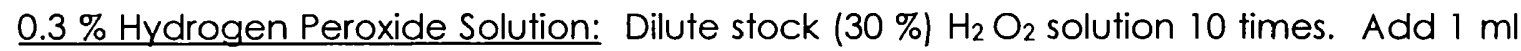
$30 \% \mathrm{H}_{2} \mathrm{O}_{2}$ to $99 \mathrm{ml}$ of d. $\mathrm{H}_{2} \mathrm{O}$.

Acetate Buffer: Mix $200 \mathrm{ml} / \mathrm{M}$ sodium acetate solution and $200 \mathrm{ml} \mathrm{d}$. $\mathrm{H}_{2} \mathrm{O}$. Add $1 \mathrm{M}$ $\mathrm{HCl}$ (up to around $190 \mathrm{ml}$ ) gradually to solution with continuous stirring whilst monitoring $\mathrm{pH}$. Stop adding $\mathrm{HCl}$ when $\mathrm{pH}$ is between 3.1 and 3.3. Store in fridge.

Rinsing Solution: Mix acetate buffer and d. $\mathrm{H}_{2} \mathrm{O}$ at a ratio of 1:19 respectively .

Dehydrating TMB containing material 
The TMB reaction product is very sensitive to exposure to alcohol therefore care has to be taken during dehydration that exposure is kept to a minimum and the solutions are appropriately buffered.

\section{Dehydration And Coverslipping}

The mounted sections are dehydrated in the following progressively stronger alcohol solutions:
A) Rinse in d. $\mathrm{H}_{2} \mathrm{O}$.
B) 10 seconds in $70 \%$ alcohol.
C) 10 seconds in $95 \%$ alcohol.
D) 10 seconds in $100 \%$ alcohol.
E) 10 seconds in absolute alcohol.
F) 5 minutes in xylene I.
G) 5 minutes in xylene II.
H) Coverslip with DPX mounting agent.

Alcohols should be diluted with acetate buffer rinsing solution (described above) for correct for correct solution $\mathrm{pH}$.

If the sections are to be counter stained with cresyl violet they should be stained for the appropriate time 2.5 - 5 minutes (depending on stains age), rinsed in d. $\mathrm{H}_{2} \mathrm{O}$ and run through the above dehydration and mounting protocol. 


\section{3,3' - Diaminobenzidine (DAB) Chromogen}

Process sections in appropriate containers through following solutions.
A) Incubate sections in DAB solution at room temp for 30 minutes.
B) Rinse in three changes 0.1 M phosphate buffer.
C) Mount onto subbed slides from fresh phosphate buffer.

\section{$\underline{\text { Solutions Required }}$}

\section{$\underline{D A B}$ Solution}

I used $100 \mathrm{ml}$ of reaction solution per reaction.

$50 \mathrm{mg}$ of $D A B$ dissolved with ultrasound in $100 \mathrm{ml} 0.1 \mathrm{M}$ phosphate buffer solution. Reaction solution is activated just before the beginning of the reaction by adding $6 \mathrm{ml}$ of $0.3 \% \mathrm{H}_{2} \mathrm{O}_{2}$.

\section{Dehydration And Coverslipping}

As the DAB reaction product is not sensitive to alcohol exposure, the sections can be dehydrated longer. The protocol is the same as for TMB material (above) but the sections should be dehydrated for at least three minutes in each alcohol. 


\section{Biotinylated Dextran Amine (BDA) Histology}

\section{Fixation}

Animals that had been injected with BDA were perfused in a similar manner to those that had been injected with WGA-HRP, except the fix contained $0.05 \%$ glutaraldehyde.

Fixation Fluids

Make up paraformaldehyde as for WGA-HRP material and add $0.05 \%$ glutaraldehyde. $0.5 \mathrm{ml}$ glutaraldehyde in $1000 \mathrm{ml}$ paraformaldehyde solution.

\section{Section Processing}

After cutting and before processing the sections are stored in phosphate buffered saline.

Phosphate Buffered Saline: Dissolve $9 \mathrm{~g}$ of $\mathrm{NaCl}$ in $1000 \mathrm{ml}$ of $0.1 \mathrm{M}$ phosphate buffer solution.

\section{Reaction}

The sections are processed through the following steps in appropriate mesh bottomed bins: 

A) Rinse sections three times for 10 minutes each in $0.1 \mathrm{M}$ phosphate buffered saline in which $0.3 \%$ of the detergent Triton has been added.
B) React the sections in $A B C$ solution for two hours with agitation.
C) Wash sections four times in $0.1 \mathrm{M}$ phosphate buffer solution 20 minutes each.
D) React sections in DAB solution for up to 15 minutes.
E) Rinse three times in $0.1 \mathrm{M}$ phosphate buffer solution, 5- 10 minutes each.
F) Mount onto subbed slides from fresh phosphate buffer solution.

\section{Reaction solutions}

ABC Solution: 5 drops of $A$ mixed with 5 drops of $B$ (from Vectastain $A B C$ kit) in $25 \mathrm{ml}$ of working solution (0.1 M phosphate buffered solution with $0.3 \%$ Triton added).

DAB Solution: As above.

Dehydration And Mounting: As described above for DAB chromogen.

\section{Fibre Stains}

Sections were processed with the following protocol to reveal myelin:

A) Place free floating sections into clean mesh bottomed bins.

B) Hydrate tissue in successively more dilute alcohols - 95\%, $80 \%, 70 \%, 50 \%$ (diluted with d. $\mathrm{H}_{2} \mathrm{O}$ ) for 5 minutes each on shaker.

C) Rinse twice in d. $\mathrm{H}_{2} \mathrm{O}$ for 5 minutes each.

D) Transfer to mordant. Leave in mordant overnight on shaker. 
E) Rinse sections in d. $\mathrm{H}_{2} \mathrm{O}$ and place in staining solution.

F) Leave in stain for $\mathrm{Thr}$ on shaker. Check under dissection microscope to determine if staining is sufficient.

G) Rinse sections in d. $\mathrm{H}_{2} \mathrm{O}$ (4/5 times) and mount onto subbed slides from d. $\mathrm{H}_{2} \mathrm{O}$, and let slides air dry.

H) Dehydrate in successive alcohols (diluted with d. $\mathrm{H}_{2} \mathrm{O}$ ) and coverslip.

\section{$\underline{\text { Solutions Required }}$}

Mordant: Unfiltered $2.5 \%$ ferric ammonium sulphate solution ( $5 \mathrm{~g}$ in $200 \mathrm{ml} \mathrm{d.} \mathrm{H}_{2} \mathrm{O}$ ).

\section{Staining Soltion:}

To $50 \mathrm{ml} \mathrm{d.} \mathrm{H}_{2} \mathrm{O}$ add $10 \mathrm{ml}$ of $1 \%$ lithium carbonate $\left(\mathrm{Li}_{2} \mathrm{CO}_{3}\right)$ solution $\left(0.5 \mathrm{~g} \mathrm{Li}_{2} \mathrm{CO}_{3}\right.$ in $50 \mathrm{ml}$ d. $\mathrm{H}_{2} \mathrm{O}$ left on shacker overnight), and $5 \mathrm{ml}$ stock haematoxylin (1 $\mathrm{g}$ haematoxylin in $10 \mathrm{ml}$ absolute alcohol).

\section{NissI Stains}

Sections were processed as follows for Nissl stain:
A) Clear in xylene -5 minutes.
B) $100 \%$ alcohol -3 minutes.
C) $95 \%$ alcohol -3 minutes.
D) $70 \%$ alcohol -3 minutes.
E) Distilled water -3 minutes.
F) Cresyl stain - 5-10 minutes depending on age of stain.
G) Distilled water - rinse. 
H) $70 \%$ alcohol - three minutes.

I) $95 \%$ alcohol - three minutes.

J) $100 \%$ alcohol - three minutes.

K) Xylene 1- five minutes.

L) Xylene II- five minutes.

M) Coverslip with DPX mounting agent

If sections were previously reacted with TMB the protocol differs (see above).

\section{Cytochrome Oxidase}

Sections are processed as follows to reveal cytochrome oxidsase:

A) Sections are placed directly into bins containing the reaction fluid, and reacted at either $37^{\circ} \mathrm{C}$ for 4 hours or overnight at $4^{\circ} \mathrm{C}$.

B) Removed from the reaction fluid and rinsed three times in $0.1 \mathrm{M}$ phosphate buffer.

C) Mount onto subbed slides from fresh 0.1 M phosphate buffer and leave to air dry.

D) Dehydrate in ascending alcohols $70 \%, 95 \%, 100 \%$, absolute acohol lat least three minutes each), clear in two changes of xylene (5 minutes).

E) Coverslip with DPX mounting agent.

\section{Solutions Required}

Reaction Fluid: In $100 \mathrm{ml}$ of $0.1 \mathrm{M}$ phosphate buffer dissolve the following using ultrasound:

i) $\quad 50 \mathrm{mg} \mathrm{DAB}$.

ii) $\quad 35 \mathrm{mg}$ cytochrome C. 
iii) $\quad 4 \mathrm{~g}$ sucrose.

iv) $20 \mathrm{mg}$ catalase. 
APPENDIX II 


\section{Other Work Carried Out During the Writing Of This Thesis}

During the writing of this thesis I spent a year in the lab of Professor Ford Ebner at Vanderbilt University in Nashville, Tennessee developing a technique to study response properties of neurones in the barrel field to whisker stimulation in the awake behaving rat. In a related series of experiments we studied the effects of enriched environmental experience on the changes seen in the response properties of barrel neurones after whisker cutting. Although these experiments were not directly related to the behavioural and anatomical studies reported in the thesis, they extended my experience in the study of this major somatosensory system in the rat.

\section{Response Properties Of Barrel Field Neurons In The Awake Behaving Rat}

Early electrophysiological studies suggested that cells within the rat whisker barrel in the PMBSF would respond only to stimulation of the whisker to which that barrel was linked (Welker, 1971). Later studies suggested that this simple story was not true under all conditions. Neurones within a barrel can respond to whiskers other than the principle whisker (Ito, 1985; Simons, 1978; Welker, 1976). The variable receptive field size is directly related to the depth and type of anaesthetic used. These factors also affect the dynamic responses of neurones in the PMBSF (Armstrong-James and Fox, 1987). Discussion has been intense as to what type of anaesthetic gives the best representation of the natural state of neuronal responses (see Simons et al., 1992; and Armstrong-James, 1995). 
It would be an advantage to be able to record from an un-anaesthetised behaving animal, an approach that has long been used in the study of primates, Evarts pioneered the study of response properties of neurones in the motor cortex, and Mountcastle in the somatosensory system of awake behaving monkeys. Simons and his colleagues (Simons et al., 1992) have recorded from un-anaesthetised rats. In their study the animals were held freely in the experimenters hands with an electrode fixed in place in the barrel field.

Working with colleagues in Ebner's lab, I helped to develop a technique in which the responses of single cells could be isolated in the PMBSF of the awake rat. In our procedure a bolt and chamber were placed onto the animals head using tiny stainless steel screws and dental cement under full surgical anaesthesia. A craniotomy had been made under the $7 \mathrm{~mm}$ opening of the chamber to expose the dura. Tungsten wires could be advanced into the brain whilst the animal's head was immobilised. Our technique had the added advantage that the animals head was still, minimising movement artefact, and allowing us to monitor whisker movement using laser based sensors (see Bermejo et al., 1996). The chamber allowed multiple entries into the brain at different sites, permitting sampling around the barrel field. Single cells could be isolated from the recording using a window discriminator, and the responses of that cell recorded and analysed using a computer with Spike II software.

Using this preparation we performed three experiments. In one we cut the animals $7^{\text {th }}$ nerve paralysing the whiskers. We could then stimulate a whisker using a controllable air puff at frequencies of $0.5,1,3,6,9,12,15$ and $18 \mathrm{~Hz}$ in an accurate, and repeatable 
manner. These results were compared to those from previous studies using the same stimulus, recording and analysis protocols in anaesthetised animals. We found that single neurones responded best to frequencies between 0.5 and $9 \mathrm{~Hz}$. At higher frequencies the number of spikes evoked decreased significantly. With stimulus frequencies less than $9 \mathrm{~Hz}$ neurons were able to follow the stimulus train. At higher frequencies neurones would respond to the air puff but they would not fire after every stimulus.

A second experiment involved the same stimulus parameters but responses were recorded with two electrodes in two different areas of the cortex. The responses were then cross correlated to look for systematic relation between them. The electrode tracts were later reconstructed to locate the exact electrode placements within the cortex. When we recorded simultaneously from two neurones in the same barrel, the responses of the cells were strongly correlated. The correlation was dependent on the frequency at which the principle whisker was stimulated. The best correlation seen between a pair of cells were at frequencies that were not the optimal frequency of either individual neurone. The position of the two cells in the barrel cortex influences the amount of cross correlation observed. If the two cells are in different barrels or in a barrel and septum the cells show weaker correlation over a restricted frequency range.

The above two experiments have been reported in preliminary form by, Sachdev, R.N.S., Jenkinson, E.W., and Ebner, F.F. Society for Neuroscience Abstracts, 24: 248.16, 1998, and Sachdev, R.N.S., Jenkinson, E.W., Melzer, P. and Ebner, F.F., Barrels XI Satellite 
Meeting of the Society for Neuroscience, Nov., 1998. Abstract: Somatosensory and Motor Research, 16: 165-166, 1999.

In a final experiment we trained animals to break a laser-based detector with its whisker for a reward of chocolate milk. In order to get the reward, the animal had to break the detector which was placed at the back of the whisker sweep and one at the very front. Thus, the animal had to execute a complete whisk before it would receive a reward. A smooth contact surface was then put in a position in front of the front sensor to record responses of the whiskers as they contacted the surface.

Short-Term Enriched Experience Potentiates Whisker-Pairing plasticity of Barrel Cortex Neurones In Adult Rats

Under normal conditions a neurone found in a barrel of the PMBSF responds best to stimulation of the whisker which that barrel represents; the principle whisker of the cell's receptive field. Cells will also respond to stimulation of surround whiskers near the principle whisker but at a longer latency and lower frequency than responses to the principle whisker. In general, cells in a given barrel respond best to the stimulation of the surround whisker with the closest barrel in the PMBSF. That is, if the cell is within the D2 barrel but on the D1 side of that barrel, it will respond to stimulation of both DI and D3 whiskers but more efficiently to DI whisker stimulation. When many cells within a 
barrel are sampled, this bias is averaged out and the population of cells as a whole is seen to respond evenly to all the surround whiskers.

The excitatory drive on a cell of the principle and non-principle whisker can be altered by cutting some of the whiskers. If all but the D1 and the D2 whiskers are trimmed to their bases and the animal is left for an appropriate amount of time, cell responses in the D2 barrel will show an increased tendency to respond to stimulation of the remaining D1 whisker. The changes seen in this paradigm reflect whisker pairing plasticity.

Similar cortical plasticity in adult animals has been seen after peripheral denervation or limb amputation in a variety of mammals. The mechanism of alteration of the cortical response to sensory input remains unclear. The whisker pairing paradigm has the advantage of altering central responses after an innocuous manipulation of peripheral sensory input and promises to help in further understanding the mechanism of plasticity in the mammalian cortex.

We investigated the possibility that the normal process of whisker pairing may occur more quickly if the animal were placed in an enriched environment rather than the standard home cage. For this experiment individual subjects were placed in an enriched environment with five other rats for two nights before recordings were made. The enriched environment consisted of a large wire cage of about $200 \mathrm{~cm}$ wide $100 \mathrm{~cm}$ tall and $75 \mathrm{~cm}$ deep. The cage was split into three levels, with runways between each, 
had two feeding platforms and was filled with plastic children's toys. On the second night at 19:00 the animal had all the vibrissae on the right side of the face apart from the D1 and D2 whiskers cut to their bases, the next morning at 09:00 they were prepared with urethane anaesthesia for single-cell recording.

In animals housed in standard home cages, 24 hours of whisker pairing produces a whisker pairing bias in the principle (D2) barrel column to stimulation of the paired surround whisker (DI) in cells in layers II and III, but not in cells within the barrel itself in layer IV (Diamond et al., 1994). Whisker pairing plasticity does occur in layer IV barrels after 65 hours in normal home cages (Diamond et al., 1993). In our experiment whisker were paired over 14 hours in the enriched environment which induced plasticity in layer IV barrel celis. The result indicates that mature cortical circuitry may be modified more rapidly under conditions that provide heightened sensory input.

The above experiment was reported in preliminary form by Rema, V., Armstrong-James, M., Jenkinson, E.W. and Ebner F.F., Society for Neuroscience Abstracts, 24: 249.5, 1998, and Rema, V., Armstrong-James, M., Jenkinson, E.W. and Ebner F.F., Barrels XI Satellite Meeting of the Society for Neuroscience, Nov., 1998. Abstract: Somatosensory and Motor research 16: 187, 1999. 


\section{REFERENCE LIST}

Adams, D. L. 1997. University Of London. 1997.

Aldes, L.D. and T.B. Boone (1985) Organization of projections from the principal sensory trigeminal nucleus to the hypoglossal nucleus in the rat: an experimental light and electron microscopic study with axonal tracer techniques. Exp.Brain Res. 59:16-29.

Andres, K.H. (1966) [On the microstructure of receptors on sinus hair]. Z.Zellforsch.Mikrosk.Anat. 75:339-365.

Andrew, R.J. (1963) The origins and evolution of the calls and facial expressions of primates. Behaviour 20:1-109.

Armstrong-James, M., J. Britto, K. Fox, and B. Mercier (1987) Short latency responses to vibrissa stimulation from layer $\mathrm{Vb}$ neurones in the rat barrel field. J.Physiol.(Lond.) $388: 43 p$

Armstrong-James, M. and C.A. Callahan (1991a) Thalamo-cortical processing of vibrissal information in the rat. II. spatiotemporal convergence in the thalamic ventroposterior medial nucleus (VPm) and its relevance to generation of receptive fields of S1 cortical "barrel" neurones. J.Comp.Neurol. 303:211-224.

Armstrong-james, M., C.A. Callahan, and M.A. Friedman (1991b) Thalamo-cortical processing of vibrissal information in the rat. I. Intracortical origins of surround but not centre-receptive fields of layer IV neurones in the rat S1 barrel field cortex. J.Comp.Neurol. 303:193-210.

Armstrong-James, M. and K. Fox (1987) Spatiotemporal convergence and divergence in the rat S1 "barrel" cortex. J.Comp.Neurol. 263:265-281.

Armstrong-James, M., K. Fox, and A. Das-Gupta (1992) Flow of excitation within rat barrel cortex on striking a single vibrissa. J Neurophysiol 68:1345-58.

Armstrong-James, M. and M.J. George (1988a) Bilateral receptive fields of cells in rat Sml cortex. Exp.Brain Res. 70:155-165. 
Armstrong-James, M. and M.J. George (1988b) Influence of anesthesia on spontaneous activity and receptive field size of single units in rat Sml neocortex. Exp.Neurol. 99:369-387.

Arvidsson, J. (1977) Retrograde axonal transport of horseradish peroxidase from cornea to trigeminal ganglion. Acta Neuropathologica 38:49-52.

Arvidsson, J. (1982) Somatotopic Organization of Vibrissae Afferents in the Trigeminal Sensory Nuclei of the Rat Studied by Transganglionic Transport of HRP. J.Comp.Neurol. $211: 84-92$.

Asanuma, C., W.T. Thach, and E.G. Jones (1983) Brainstem and spinal projections of the deep cerebellar nuclei in the monkey, with observations on the brainstem projections of the dorsal column nuclei. Brain Res. 286:299-322.

Azizi, S.A., G.A. Mihailoff, R.A. Burne, and D.J. Woodward (1981) The pontocerebellar system in the rat: an HRP study. I. Posterior vermis. J.Comp.Neurol. 197:543-8.

Baker, J., A. Gibson, M. Glickstein, and J. Stein (1976) Visual cells in the pontine nuclei of the cat. J.Physiol.(Lond.) 255:415-33.

Baker, J., A. Gibson, G. Mower, F. Robinson, and M. Glickstein (1983) Cat visual corticopontine cells project to the superior colliculus. Brain Res. 265:227-32.

Barth, T.M. and T. Schallert (1987) Somatosensorimotor function of the superior colliculus, somatosensory cortex, and lateral hypothalamus in the rat. Exp.Neurol. 95:661678.

Bates, C.A. and H.P. Killackey (1985) The organization of the neonatal rat's brainstem trigeminal complex and its role in the formation of central trigeminal patterns. J.Comp.Neurol. 240:265-87.

Batton, R.R., A. Jayaraman, D. Ruggiero, and M.B. Carpenter (1977) Fastigial efferent projections in the monkey: an autoradiographic study. J.Comp.Neurol. 174:281305.

Beaudoin, A.R. (1980) Embryology and teratology. In H.J. Baker, J.R. Lindsey, and S.H. Weisbroth (eds): The Laboratory Rat. New York: Academic Press, pp. 75-101. 
Beck, E. (1950) The origin, course, and termination of the prefrontal to pontine tract in the human brain. Brain 73:368-391.

Belford, G.R. and H.P. Killackey (1979) Vibrissae representation in subcortical trigeminal centers of the neonatal rat. J.Comp.Neurol. 183:305-21.

Bennett-Clarke, C.A., N.L. Chiaia, M.F. Jacquin, and R.W. Rhoades (1992) Parvalbumin and calbindin immunocytochemistry reveal functionally distinct cell groups and vibrissa-related patterns in the trigeminal brainstem complex of the adult rat. J.Comp.Neurol. 320:323-38.

Bermejo, R., M. Harvey, P. Gao, and H.P. Zeigler (1996) Conditioned whisking in the rat. Somatosens.Mot.Res. 13:225-33.

Bernard, J.F. (1987) Topographical organisation of olivocerebellar and corticonuclear connections in the rat - an WGA-HRP study: I. Lobules IX, X and flocculus. J.Comp.Neurol. 241-258.

Brecht, M., B. Preilowski, and M.M. Merzenich (1997) Functional architecture of the mystacial vibrissae. Behav.Brain Res. 84:81-97.

Bremer, F. (1956) Arch.Suisses.Neurol.Psychiatr. 78:31-87.

Brinkman, J. and H.G. Kuypers (1972) Splitbrain monkeys: cerebral control of ipsilateral and contralateral arm, hand, and finger movements. Science 176:536-539.

Brodal, P. (1972a) The corticopontine projection from the visual cortex in the cat. I. The total projection and the projection from area 17. Brain Res. 39:297-317.

Brodal, P. (1972b) The corticopontine projection from the visual cortex in the cat. II. The projection from areas 18 and 19. Brain Res. 39:319-35.

Brodal, P. (1978) The corticopontine projection in the rhesus monkey. Origin and principles of organization. Brain 101:251-83.

Brodal, P., E. Dietrichs, J.G. Bjaalie, T. Nordby, and F. Walberg (1983) Is lectin-coupled horseradish peroxidase taken up and transported by undamaged fibers as well as by damaged fibers in the central nervous system. Brain Res. 1-9. 
Broughton, S.D. (1823) On the Use of the Whiskers in Feline and other Animals. London Medical and Physical Journal 49:397-398.

Bruce, L.L., J.G. McHaffie, and B.E. Stein (1987) The organization of trigeminotectal and trigeminothalamic neurons in rodents: a double-labeling study with fluorescent dyes. J.Comp.Neurol. 262:315-30.

Buchbinder, S., B. Dixon, Y.W. Hwang, J.G. May, and M. Glickstein (1980) The effects of cortical lesions on visual guisdance of the hand. Society for Neuroscience Abstracts 6:675(Abstract)

Bucy, P.C., R. Ladpli, and A. Ehrlich (1966) Destruction of the pyramidal tract in the monkey. J.Neurosurg. 25:1-23.

Bugbee, N.M. and B.S. Eichelman (1972) Sensory alterations and aggressive behavior in the rat. Physiol Behav 8:981-5.

Burne, R.A., G.A. Mihailoff, and D.J. Woodward (1978) Visual corticopontine input to the paraflocculus: a combined autoradiographic and horseradish peroxidase study. Brain Res. 143:139-46.

Cajal, S.R.Y. (1909) Histologie du Systeme Nerveux de l'Homme et des Vertebres. Madrid: C.S.I.C.

Carey, D.P., L.A. Booth, and M.A. Goodale (1987) Can gerbils with lesions of primary visual cortex use retinal image size to estimate distance in a jumping task? Society for Neuroscience Abstracts 13:629(Abstract)

Carvell, G.E., S.A. Miller, and D.J. Simons (1996) The relationship of vibrissal motor cortex unit activity to whisking in the awake rat. Somatosens.Mot.Res. 13:1 15-27.

Carvell, G.E. and D.J. Simons (1990) Biometric analyses of vibrissal tactile discrimination in the rat. J Neurosci 10:2638-48.

Chalupa, L.M. and R.W. Rhoades (1977) Responses of visual, somatosensory, and auditory neurones in the golden hamster's superior colliculus. J.Physiol.(Lond.) 270:595-626. 
Chiaia, N.L., R.W. Rhoades, C.A. Bennett-Clarke, S.E. Fish, and H.P. Killackey (1991a) Thalamic processing of vibrissal information in the rat. I. Afferent input to the medial ventral posterior and posterior nuclei. J.Comp.Neurol. 314:201-16.

Chiaia, N.L., R.W. Rhoades, S.E. Fish, and H.P. Killackey (1991b) Thalamic processing of vibrissal information in the rat: II. Morphological and functional properties of medial ventral posterior nucleus and posterior nucleus neurons. J.Comp.Neurol. 314:217-36.

Chmielowska, J., G.E. Carvell, and D.J. Simons (1989) Spatial organization of thalamocortical and corticothalamic projection systems in the rat Sml barrel cortex. J.Comp.Neurol. 285:325-38.

Cicerone, C.M. (1976) Cones survive rods in the light-damaged eye of the albino rat. Science 194:1183-1185.

Classen, J., E. Kunesch, F. Binkofski, F. Hilperath, G. Schlaug, R.J. Seitz, M. Glickstein, and H.J. Freund (1995) Subcortical origin of visuomotor apraxia [published erratum appears in Brain 1996 Apr;1 19(Pt 2):697]. Brain 118:1365-74.

Courville, J. and J.A. Saint-Cyr (1978) Modification of the horseradish peroxidase method avoiding fixation. Brain Res. 551-558.

Crandall, J.E., M. Korde, and V.S. Caviness, Jr. (1986) Somata of layer V projection neurons in the mouse barrelfield cortex are in preferential register with the sides and septa of the barrels. Neurosci.Lett. 67:19-24.

Danforth, C.H. (1925) Hair in its relation to questions of homology and phylogeny. American Journal of Anatomy 36:47-68.

Darian-Smith, I. (1973) The Trigeminal System. In A. Iggo (ed): Somatosensory System. Berlin: Springer-Verlag, pp. 271-314.

Darian-Smith, I. (1984) The sense of touch: performance and peripheral neural processes. In I. Darian-Smith (ed): Vol. III. Sensory processes. American Society of Physiology, pp. 739-787. 
Darian-Smith, I. and G. Mayday (1960) Somatotopic organisation within the brainstem trigeminal complex of the cat. Exp.Neurol. 2:290-309.

Dean, P. (1976) Effects of inferotemporal lesions on the behavior of monkeys. Psychol Bull 83:41-71.

Dean, P., P. Redgrave, N. Sahibzada, and K. Tsuji (1986) Head and body movements produced by electrical stimulation of superior colliculus in rats: effects of interruption of crossed tectoreticulospinal pathway. Neuroscience 19:367-380.

Desimone, R., T.D. Albright, C.G. Gross, and C. Bruce (1984) Stimulus-selective properties of inferior temporal neurons in the macaque. J.Neurosci. 4:2051-62.

Déjérine, J. (1901) Anatomie des Centres Nerveux. Paris: Rueff.

Diamond, M.E., M. Armstrong-James, M.J. Budway, and F.F. Ebner (1992a) Somatic sensory responses in the rostral sector of the posterior group (POm) and in the ventral posterior medial nucleus (VPM) of the rat thalamus: dependence on the barrel field cortex. J.Comp.Neurol. 319:66-84.

Diamond, M.E., M. Armstrong-James, and F.F. Ebner (1992b) Somatic sensory responses in the rostral sector of the posterior group (POm) and in the ventral posterior medial nucleus (VPM) of the rat thalamus. J.Comp.Neurol. 318:462-76.

Diamond, M.E., M. Armstrong-James, and F.F. Ebner (1993) Experience-dependent plasticity in adult rat barrel cortex. Proc Natl Acad Sci U S A 90:2082-6.

Diamond, M.E., W. Huang, and F.F. Ebner (1994) Laminar comparison of somatosensory cortical plasticity. Science 265:1885-1888.

Donoghue, J.P. and S.T. Kitai (1981) A collateral pathway to the neostriatum from corticofugal neurons of the rat sensory-motor cortex: an intracellular HRP study. J.Comp.Neurol. 201:1-13.

Dorfl, J. (1982) The musculature of the mystacial vibrissae of the white mouse. J.Anat. 135:147-54.

Dorfl, J. (1985) The innervation of the mystacial region of the white mouse. A topographical study. J.Anat. 142:173-184. 
Downer, J.d.C. (1959) Changes in visually guided behaviour following mid-sagittal division of optic chiasm and corpus collosum in the monkey (Macaca mulatta). Brain 82:251-259.

Downer, J.d.C. (1958) Federation Proceedings 17:37

Dräger, U.C. (1975a) Receptive fields of single cells and topography in mouse visual cortex. J.Comp.Neurol. 160:269-90.

Dräger, U.C. and D.H. Hubel (1975b) Physiology of visual cells in mouse superior colliculus and correlation with somatosensory and auditory input. Nature 253:203-4.'

Dreher, B., A.J. Sefton, S.Y. Ni, and G. Nisbett (1985) The morphology, number. distribution and central projections of Class I retinal ganglion cells in albino and hooded rats. Brain Behav. Evol. 26:10-48.

Drooglever Fortuijn, A.B. (1914) Cortical cell-lamination of the hemispheres of some rodents. Archives of Neurological Psychiatry 6:221-354.

Drooglever Fortuijn, J. (1938) The systematic arrangement of fibres in capsula interna, thalamus opticus, and pes pedunculi. Proc Kon Nederl Acad Wet 41:3-12.

Dursteler, M.R., C. Blakemore, and L.J. Garey (1977) Uptake of horseradish peroxidase by geniculo-cortical axons in the golden hampster: analysis by computer reconstruction. Exp.Brain Res. 487-500.

Dykes, R.W. (1975) Afferent fibers from mystacial vibrissae of cats and seals. J Neurophysiol 38:650-62.

Ellard, C.G., M.A. Goodale, D.M. Scorfield, and C. Lawrence (1986) Visual cortical lesions abolish the use of motion parallax in the Mongolian gerbil. Exp.Brain Res. 64:599602.

Ellard, C.G., M.A. Goodale, and B. Timney (1984) Distance estimation in the Mongolian gerbil: the role of dynamic depth cues. Behav.Brain Res. 14:29-39.

Erzurumlu, R.S., C.A. Bates, and H.P. Killackey (1980) Differential organization of thalamic projection cells in the brain stem trigeminal complex of the rat. Brain Res. 198:427-33. 
Erzurumlu, R.S. and H.P. Killackey (1979) Efferent connections of the brainstem trigeminal complex with the facial nucleus of the rat. J.Comp.Neurol. 188:75-86.

Ettlinger, G. and H.B. Morton (1963) Callosal section: its effects on performance of bimanual skill. Science 139:485-486.

Exner, S. and J. Paneth (1889) Arch.Physiol. 44:544-555.

Fabri, M. and H. Burton (1991) Topography of connections between primary somatosensory cortex and posterior complex in rat: a multiple fluorescent tracer study. Brain Res. 538:351-7.

Fitzgerald, O. (1940) Discharges from the sensory organs of the cat's vibrissae and the modification of their activity by ions. J.Physiol.(Lond.) 98:163-178.

Flory, R.K., R. Ulrich, and P.C. Wolf (1965) The effects of visual impairment on on aggresive behavior. Psychological Record 15:185-190.

Forbes, D.J. and C. Welt (1981) Neurogenesis in the trigeminal ganglion of the albino rat: a quantitative autoradiographic study. J.Comp.Neurol. 199:133-147.

Friedberg, M. F. Receptive Field and Behavioural Analysis of Trigeminal Somesthesis. 1991. Brown University. 1991.

Fritsch, G. and E. Hitzig (1870) Uber die elektriche Erregbarkeit des Groshirns. Arch.Anat.Physiol.wissesschaftl Med. 300-332.

Fukushima, T. and F.W. Kerr (1979) Organization of trigeminothalamic tracts and other thalamic afferent systems of the brainstem in the rat: presence of gelatinosa neurons with thalamic connections. J.Comp.Neurol. 183:169-84.

Gibson, A., J. Baker, G. Mower, and M. Glickstein (1978) Corticopontine cells in area 18 of the cat. J Neurophysiol 41:484-95.

Gibson, A.R., D.I. Hansma, J.C. Houk, and F.R. Robinson (1984) A sensitive low artifact TMB procedure for the demonstration of WGA-HRP in the CNS. Brain Res. 298:235-241. 
Gibson, J.M. (1987) A quantitative comparison of stimulus-response relationships of vibrissa-activated neurons in subnuclei oralis and interpolaris of the rat's trigeminal sensory complex: receptive field properties and threshold distributions. Somatosens.Res. 5:135-55.

Gibson, J.M. and W.I. Welker (1983a) Quantitative studies of stimulus coding in first-order vibrissa afferents of rats. 1. Receptive field properties and threshold distributions. Somatosensory Research $1: 51-67$.

Gibson, J.M. and W.I. Welker (1983b) Quantitative studies of stimulus coding in first-order vibrissa afferents of rats. 2. Adaptation and coding of stimulus parameters. Somatosensory Research 1:95-117.

Glassman, R.B. (1994) Behavioral effects of SI versus SIl cortex ablations on tactile orientation-localization and postural reflexes of rats. Exp.Neurol. 125-133.

Glickstein, M., S. Buchbinder, and J.L. May (1998) Visual control of the arm, the wrist and the fingers: pathways through the brain. Neuropsychologia. 36:981-1001.

Glickstein, M., J.L. Cohen, B. Dixon, A. Gibson, M. Hollins, E. Labossiere, and F. Robinson (1980) Corticopontine visual projections in macaque monkeys. J.Comp.Neurol. 190:209-29.

Glickstein, M., N. Gerrits, I. Kralj-Hans, B. Mercier, J. Stein, and J. Voogd (1994) Visual pontocerebellar projections in the macaque. J.Comp.Neurol. 349:51-72.

Glickstein, M., I. Kralj-Hans, C. Legg, B. Mercier, M. Ramna-Rayan, and E. Vaudano (1992) The organisation of fibres within the rat basis pedunculi. Neurosci.Lett. 135:75-9.

Glickstein, M., J. May, and B. Mercier (1990) Visual corticopontine and tectopontine projections in the macaque. Arch Ital Biol 128:273-93.

Glickstein, M., J.G.d. May, and B.E. Mercier (1985) Corticopontine projection in the macaque: the distribution of labelled cortical cells after large injections of horseradish peroxidase in the pontine nuclei. J.Comp.Neurol. 235:343-59. 
Glickstein, M. and R.W. Sperry (1960) Intermanual somesthetic transfer in split-brain rhesus monkeys. J.Comp.Physiol.Psych. 53:322-327.

Glickstein, M. and R.W. Sperry (1963) Visual-motor co-ordination in the monkeys after optic tract section and commissurotomy. Federation Proceedings 22:456

Glickstein, M. and J. Stein (1991) Paradoxical movement in Parkinson's disease. Trends Neurosci 14:480-2.

Glickstein, M., J. Stein, and R.A. King (1972) Visual input to the pontine nuclei. Science 178:1110-1.

Glickstein, M. and D. Whitteridge (1987) Tatsuji Inouye and the mapping of the visual fields on the human cerebral cortex. Trends in Neuroscience 10:350-353.

Gonatas, N.K., C. Harper, T. Mizutani, and J.O. Gonatas (1979) Superior sensitivity of conugates of horse radish peroxidase with wheat germ agglutinin for studies of retrograde transport. J Histochem Cytochem 728-734.

Good, K.E. and H.P. Killackey (1991) Differential laminar distribution of corticothalamic projection neurons in rat primary somatosensory cortex. Society for Neuroscience Abstracts 17:624(Abstract)

Goodale, M.A., C.G. Ellard, and L. Booth (1990) The role of image size and retinal motion in the computation of absolute distance by the Mongolian gerbil (Meriones unguiculatus). Vision Res. 30:399-413.

Goodale, M.A., N.P. Foreman, and A.D. Milner (1978) Visual orientation in the rat: a dissociation of deficits following cortical and collicular lesions. Exp.Brain Res. 31:445-457.

Gottschaldt, K.M. and C. Vahle-Hinz (1981) Merkel cell receptors: structure and transducer function. Science 214:183-186.

Gould, B.B. (1980) Organization of afferents from the brain stem nuclei to the cerebellar cortex in the cat. Adv.Anat.Embryol.Cell Biol. 62:v-viii, 1-90.

Grant, G. and J. Arvidsson (1975) Transganglionic degeneration in trigeminal primary sensory neurons. Brain Res. 95:265-79. 
Gregg, J.M. and A.D. Dixon (1973) Somatotopic organization of the trigeminal ganglion in the rat. Arch Oral Biol 18:487-98.

Gregoire, S.E. and D.E. Smith (1975) Mouse-killing in the rat: effects of sensory deficits on attack behaviour and stereotyped biting. Anim Behav 23:186-91.

Griffiths, W.J. (1960) Responses of wild and domestic rats to forced swimming. Psychological reports 6:39-49.

Gross, C.G., C.E. Rocha-Miranda, and D.B. Bender (1972) Visual properties of neurons in inferotemporal cortex of the Macaque. J.Neurophysiol. 35:96-111.

Guic-Robles, E., C. Valdivieso, and G. Guajardo (1989) Rats can learn a roughness discrimination using only their vibrissal system. Behav Brain Res 31:285-9.

Haarsten, A.B. and W.J.C. Verhaart (1967) Cortical projections to brain stem and spinal cord in the goat by way of the pyramidal tract and the bundle of Bagley. J.Comp.Neurol. 129:189-201.

Haaxma, R. and H. Kuypers (1975) Brain 98:239-260.

Halata, Z. and B.L. Munger (1980) Sensory nerve endings in rhesus monkey sinus hairs. J.Comp.Neurol. 192:645-663.

Halsband, U. and R. Passingham (1982) The role of premotor and parietal cortex in the direction of action. Brain Res. 240:368-72.

Hartmann, F. and W. Trendelenberg (1927) Z.Gesamte Neurol.Psychiatr. 47:1449

Heidenhain, M. Über kern und protoplasma. 118. 1892. Festschr. für Kölliker.

Henschen, S.E. (1890) Klinische und anatomische Beitrage zur Pathologie des Gehirns (Pt. 1). Almquist and Wiksell.

Herkenham, M. (1980) Laminar organization of thalamic projections to the rat neocortex. Science 207:532-5. 
Hersch, S.M. and E.L. White (1982) A quantitative study of the thalamocortical and other synapses in layer IV of pyramidal cells projecting from mouse Sml cortex to the caudate- putamen nucleus. J.Comp.Neurol. 211:217-25.

Holmes, G. and W.T. Lister (1916) Disturbances of vision from cerebral lesions with special reference to the cortical representation of the macula. Brain 39:34-73.

Hoogland, P.V., E. Welker, and H. Van der Loos (1987) Organization of the projections from barrel cortex to thalamus in mice studied with Phaseolus vulgarisleucoagglutinin and HRP. Exp.Brain Res. 68:73-87.

Hore, J., J. Meyer-Lohmann, and V.B. Brooks (1977) Basal ganglia cooling disables learned arm movements of monkeys in the absence of visual guidance. Science 195:584-586.

Hore, J. and T. Vilis (1980) Arm movement performance during reversible basal ganglia lesions in the monkey. Exp.Brain Res. 39:217-228.

Huerta, M.F., A. Frankfurter, and J.K. Harting (1983) Studies of the principal sensory and spinal trigeminal nuclei of the rat: projections to the superior colliculus, inferior olive, and cerebellum. J.Comp.Neurol. 220:147-67.

Hutchins, B. and J.T. Weber (1983) A rapid myelin stain for frozen sections: modification of the Heidenhain procedure. J Neurosci Methods 7:289-94.

Hutson, K.A. and R.B. Masterton (1986) The Sensory Contribution of a Single Vibrissa's Cortical Barrel. Journal of Neurophysiology 56:1 196-1223.

Inouye, T. Die sehstörungen bei Schussverletzungen der kortikalen Sehsphäre nach Beobachtungen an Versundeten der letzten Japanische Kriege. 1909. Leipzig, W.Engelmann.

Ito, M. (1985) Processing of vibrissa sensory information within the rat neocortex. J.Neurophysiol. 46:705-715.

Ito, M. (1988) Response properties and topography of vibrissa-sensitive VPM neurons in the rat. J Neurophysiol 60:1 181-97. 
Ito, M. (1992) Simultaneous visualization of cortical barrels and horseradish peroxidaseinjected layer 5b vibrissa neurones in the rat. J.Physiol.(Lond.) 454:247-65.

Jacobs, G.H., J. Neitz, and J.F. Deegan (1991a) A retinal receptor in rodents maximally sensitve to ultraviolet lights. Invest.Opthalmol.Vis.Sci. 32:698(Abstract)

Jacobs, G.H., J. Neitz, and J.F. Deegan (1991b) Retinal receptors in rodents maximally sensitive to ultraviolet light. Nature 353:655-656.

Jacquin, M.F., M. Barcia, and R.W. Rhoades (1989) Structure-function relationships in rat brainstem subnucleus interpolaris: IV. Projection neurons. J.Comp.Neurol. 282:4562.

Jacquin, M.F., N.L. Chiaia, J.H. Haring, and R.W. Rhoades (1990) Intersubnuclear connections within the rat trigeminal brainstem complex. Somatosens.Mot.Res. 7:399-420.

Jacquin, M.F., J. Golden, and W.M. Panneton (1988) Structure and function of barrel 'precursor' cells in trigeminal nucleus principalis. Brain Res. 471:309-14.

Jacquin, M.F., J. Golden, and R.W. Rhoades (1989) Structure-function relationships in rat brainstem subnucleus interpolaris: III. Local circuit neurons. J.Comp.Neurol. 282:24-44.

Jacquin, M.F., J.S. McCasland, T.A. Henderson, R.W. Rhoades, and T.A. Woolsey (1993) 2-DG uptake patterns related to single vibrissae during exploratory behaviors in the hamster frigeminal system. J.Comp.Neurol. 332:38-58.

Jacquin, M.F., R.D. Mooney, and R.W. Rhoades (1986) Morphology, response properties, and collateral projections of trigeminothalamic neurons in brainstem subnucleus interpolaris of rat. Exp.Brain Res. 61 :457-68.

Jacquin, M.F. and R.W. Rhoades (1990) Cell structure and response properties in the trigeminal subnucleus oralis. Somatosens.Mot.Res. 7:265-88.

Jensen, K.F. and H.P. Killackey (1987) Terminal arbors of axons projecting to the somatosensory cortex of the adult rat. I. The normal morphology of specific thalamocortical afferents. J Neurosci 7:3529-43. 
Jones, E.G. (1975) Possible determinants of the degree of retrograde neuronal labeling with horseradish peroxidase. Brain Res. 249-253.

Jones, E.G. and B.K. Hartman (1978) Recent advances in neuroanatomical methodology. Annu.Rev.Neurosci. 215-296.

Karli, P. (1956) The Nonway rat's killing response to the white mouse, a experimental analysis. Behaviour 11:593-602.

Kassel, J. (1982) Somatotopic organization of SI corticotectal projections in rats. Brain Res. 231:247-55.

Keizer, K., H.G. Kuypers, and H.K. Ronday (1987) Branching cortical neurons in cat which project to the colliculi and to the pons: a retrograde fluorescent double-labeling study. Exp.Brain Res. 67:1-15.

Keller, A., E.L. White, and P.B. Cipolloni (1985) The identification of thalamocortical axon terminals in barrels of mouse Sml cortex using immunohistochemistry of anterogradely transported lectin (Phaseolus vulgaris-leucoagglutinin). Brain Res. 343:159-65.

Kievit, J. , and H.G.J.M. Kuypers (1972) Fastigial cerebellar projections to the venttrolateral nucleus of the thalamus and the organization of the descending pathways. In T. Frigyesi, E. Rinvik, and M.D. Yahr (eds): Corticothalamic Projections and Sensorimotor Activities. New York: Raven Press, pp. $91-114$.

Killackey, H.P. (1973) Anatomical evidence for cortical subdivisions based on vertically discrete thalamic projections from the ventral posterior nucleus to cortical barrels in the rat. Brain Res. 51:326-31.

Killackey, H.P., G. Belford, R. Ryugo, and D. Ryugo (1976) Anomolous organization of thalamocortical projections consequent to vibrissae removal in the newborn rat and mouse. Brain Res. 104:309-315.

Killackey, H.P. and R.S. Erzurumlu (1981) Trigeminal projections to the superior colliculus of the rat. J.Comp.Neurol. 201:221-42. 
Killackey, H.P., K.A. Koralek, N.L. Chiaia, and R.W. Rhoades (1989) Laminar and areal differences in the origin of the subcortical projection neurons of the rat somatosensory cortex. J.Comp.Neurol. 282:428-45.

Koralek, K.A., K.F. Jensen, and H.P. Killackey (1988) Evidence for two complementary patterns of thalamic input to the rat somatosensory cortex. Brain Res. 463:34651.

Koralek, K.A., J. Olavarria, and H.P. Killackey (1990) Areal and laminar organization of corticocortical projections in the rat somatosensory cortex. J.Comp.Neurol. 299:133-50.

Kossut, M. and P. Hand (1984) The development of the vibrissal cortical column: a 2deoxyglucose study in the rat. Neurosci.Lett. 46:1-6.

Krieg, W.J.S. (1947) Connections of the cerebral cortex, I. The albino rat c. extrinsic connections. J.Comp.Neurol. 86:267-394.

Kristensson, K. and Y. Olsson (1971) Retrograde axonal transport of protein. Brain Res. 29:363-365.

Kuypers, H.G.J.M., M.K. Schwarzbart, M. Miskin, and H.E. Rosvold (1965) Occipitotemporal corticocortical connections in the rhesus monkey. Exp.Neurol. $11: 245-262$.

Land, P.W., S.A. Buffer, Jr., and J.D. Yaskosky (1995) Barreloids in adult rat thalamus: three-dimensional architecture and relationship to somatosensory cortical barrels. J.Comp.Neurol. 355:573-88.

Land, P.W. and D.J. Simons (1985) Cytochrrome oxidase staining in the rat Sml barrel cortex. J.Comp.Neurol. 238:225-235.

Lansdell, H. (1968) Effect of extent of temporal lobe ablations on two lateralized deficits. Physiol.Behav. 3:271-273.

Larsson, K. and B.R. Komisaruk (1972) Abnormally fast vibrissa movements induced by tetrabenazine in rats. Psychopharmacologia 23:300-304. 
Lawrence, D.G. and H.G. Kuypers (1968a) The functional organization of the motor system in the monkey. I. The effects of bilateral pyramidal lesions. Brain 91:1-14.

Lawrence, D.G. and H.G. Kuypers (1968b) The functional organization of the motor system in the monkey. II. The effects of lesions of the descending brain-stem pathways. Brain 91:15-36.

Lee, K.J. and T.A. Woolsey (1975) A proportional relationship between peripheral innervation density and cortical neuron number in the somatosensory system of the mouse. Brain Res. 99:349-53.

Legg, C.R. and S. Lambert (1990) Distance estimation in the hooded rat: experimental evidence for the role of motion cues. Behav.Brain Res. 41:11-20.

Legg, C.R., B. Mercier, and M. Glickstein (1989) Corticopontine projection in the rat: the distribution of labelled cortical cells after large injections of horseradish peroxidase in the pontine nuclei. J.Comp.Neurol. 286:427-41.

Lichtenstein, S.H., G.E. Carvell, and D.J. Simons (1990) Responses of rat trigeminal ganglion neurons to movements of vibrissae in different directions. Somatosens.Mot.Res. 7:47-65.

Linden, R. and V.H. Perry (1983) Massive retinotectal projection in rats. Brain Res. 272:145-149.

Lorente de Nó, R. (1922) La corteza del raton, Trab. Lab. Invest.Biol. 20:78

LU, S.M. and R.C. Lin (1993) Thalamic afferents of the rat barrel cortex: a light- and electron- microscopic study using Phaseolus vulgaris leucoagglutinin as an anterograde tracer. Somatosens.Mot.Res. 10:1-16.

Lyne, A.G. (1959) The systematic and adaptive significance of the vibrissae in the Marsupialia. Proceedings of the Zoological Society of London 133:79-133.

Ma, P.M. (1991) The barrelettes--architectonic vibrissal representations in the brainstem trigeminal complex of the mouse. I. Normal structural organization. J.Comp.Neurol. 309:161-99. 
Ma, P.M. and T.A. Woolsey (1984) Cytoarchitectonic correlates of the vibrissae in the medullary trigeminal complex of the mouse. Brain Res. 306:374-9.

Mantle-St.John, L.A. and D.J. Tracey (1987) Somatosensory nuclei in the brainstem of the rat: independent projections to the thalamus and cerebellum. J.Comp.Neurol. 255:259-71.

Marchi, v. and G. Algeri (1885) Salle degenerazion; discondenti onsecutive a lesioni sperimentale in diverse zone della corteccia cerebrale. Riv.per.Frenatria Med.legal. Il: 492-494, 12: 208-252:848-850.

Marfurt, C.F. (1981) The central projections of trigeminal primary afferent neurons in the cat as determined by the tranganglionic transport of horseradish peroxidase. J.Comp.Neurol. 203:785-98.

Marin, O. and J. Angevine (1962) Topographical organisation of the lateral segmant of the basisi peduncli in man. J.Comp.Neurol. 118:165-183.

Mark, R.F. and R.W. Sperry (1968) Bimanual coordination in monkeys. Exp.Neurol. 21:92104.

Marshall, W.H., C.N. Woolsey, and P. Bard (1941) Observations on cortical sensory mechanisms of cat and monkey. J.Neurophysiol. 4:25-43.

Martin, X. and M. Dolivo (1983) Neuronal and transneuronal tracing in the trigeminal system of the rat using the herpes virus suis. Brain Res. 273:253-76.

McCasland, J.S. and T.A. Woolsey (1988) High-resolution 2-deoxyglucose mapping of functional cortical columns in mouse barrel cortex. J.Comp.Neurol. 278:555-69.

MCHaffie, J.G. and B. Stein (1981) Properties of cells activated by vibrissal movement in the superior colliculus of the rodent. Society for Neuroscience Abstracts 7:393(Abstract)

Menétrey, D., J. de Pommery, K.G. Baimbridge, and M. Thomasset (1992) CalbindinD28K (CaBP28k)-like immunoreactivity in ascending projections. I. Trigeminal nucleus caudalis and dorsal vagus complex projections. European Journal of Neuroscience 4:61-69. 
Mercier, B. E. 1989. University College London. 1989.

Mercier, B.E., C.R. Legg, and M. Glickstein (1990) Basal ganglia and cerebellum receive different somatosensory information in rats. Proc Natl Acad Sci U S A 87:4388-92.

Mesulam, M.M. (1982) Tracing neural connections with horseradish peroxidase. New York: Wiley.

Mihailoff, G.A. (1983) Intra- and interhemispheric collateral branching in the rat pontocerebellar system, a fluorescence double-label study. Neuroscience 10:141-60.

Mihailoff, G.A., R.A. Burne, and D.J. Woodward (1978) Projections of the sensorimotor cortex to the basilar pontine nuclei in the rat: an autoradiographic study. Brain Res. 145:347-54.

Mihailoff, G.A., H. Lee, C.B. Watt, and R. Yates (1985) Projections to the basilar pontine nuclei from face sensory and motor regions of the cerebral cortex in the rat. J.Comp.Neurol. 237:251-63.

Mihailoff, G.A., C.B. Watt, and R.A. Burne (1981) Evidence suggesting that both the corticopontine and cerebellopontine systems are each composed of two separate neuronal populations: an electron microscopic and horseradish peroxidase study in the rat. J.Comp.Neurol. 195:221-42.

Milner, A.D., E.M. Ockleford, and W. Dewar (1977) Visuo-spatial performance following posterior parietal and lateral frontal lesions in stumptail macaques. Cortex 13:350-60.

Milner, B. (1958) Psychological defects produced by temporal lobe excision. Res.Publ.Assoc.Res.Nerv.Ment.Dis. 36:244-257.

Mosconi, T.M., F.L. Rice, and M.J. Song (1993) Sensory innervation in the inner conical body of the vibrissal follicle- sinus complex of the rat. J.Comp.Neurol. 328:232-51.

Munger, B.L. and Z. Halata (1983) The sensory innervation of primate facial skin. I. Hairy skin. Brain Res. 286:45-80. 
Munk, H. Über die Functionen der Grosshirnrinde. English Translation, 28-53. 1881. Berlin, Hirschwald.

Munk, H. (1896) Sitzungber.K.Preuss.Akad.Wiss. Nov.5:

Myers, R.E. (1956) Brain 19:358

Myers, R.E., R.W. Sperry, and N.M. McCurdy (1962) Neural mechanisms in visual guidance of limb movements. Arch.Neurol. 7:195-202.

Nauta, W.J.H. and P.A. Gygax (1954) Silver impregnation of degenerating axons in the central nervous system: a modified technic. Stain Technology 29:91-93.

Neitz, J. and G.H. Jacobs (1986) Reexamination of spectral mechanisms in the rat (Rattus norvegicus). J Comp.Psychol. 100:21-29.

Newcombe, F. and W.R. Russell (1969) Dissociated visual perceptual and spatial deficits in focal lesions of the right hemisphere. Journal of Neurology Neurosurgery and Psychiatry 32:72-81.

Newsome, W.T. and R.H. Wurtz (1982) Identification of architectonic zones containing visual tracking cells in the superior temporal sulcus (STS) of macaque monkeys. Invest.Opthal.Vis.Sci.Supp. 22:238

Nord, S.G. (1967) Somatotopic organization in the spinal trigeminal nucleus, the dorsal column nuclei and related structures in the rat. J.Comp.Neurol. 130:343-56.

Nord, S.G. (1968) Receptor field characteristics of single cells in the rat spinal trigeminal complex. Exp.Neurol. $21: 236-243$.

Nyby, O. and J. Jensen (1951) An experimental investigation of the cortico-pontine projection in Macaca mulata. Skr.Nor.Vidensk.Akad.Oslo I Mat.Naturvidensk.KI. 3:1-47.

Pandya, D.N. and H.G.J.M. Kuypers (1969) Cortico-cortical connections in the rhesus monkey. Brain Res. 13:13-36. 
Patrick, G.W. and M.A. Robinson (1987) Collateral projections from trigeminal sensory nuclei to ventrobasal thalamus and cerebellar cortex in rats. J.Morphol. 192:229236.

Paxinos, G. , and C. Watson (1982) The rat brain in stereotaxic coordinates. Academic Press.

Pellegrino, L.J., A.S. Pellegrino, and A.J. Cushman (1979) A stereotaxic atlas of the rat brain. New York: Plenum.

Peschanski, M. (1984) Trigeminal afferents to the diencephalon in the rat. Neuroscience 12:465-87.

Peterson, B.E. and D. Goldreich (1994) A new approach to optical imaging applied to rat barrel cortex. J Neurosci Methods 54:39-47.

Phelan, K.D. and W.M. Falls (1989) An analysis of the cyto- and myeloarchitectonic organization of trigeminal nucleus interpolaris in the rat. Somatosens.Mot.Res. 6:333-66.

Pocock, R.I. (1914) On the Facial Vibrissae of Mammalia. Proceedings of the Zoological Society of London 60:889-912.

Pohl, W. (1973) Dissociation of spatial discrimination deficits following frontal and parietal lesions in monkeys. J.Comp.Physiol.Psychol. 82:227-39.

Pubols, B.H., Jr., P.J. Donovick, and L.M. Pubols (1973) Opossum trigeminal afferents associated with vibrissa and rhinarial mechanoreceptors. Brain Behav.Evol. $7: 360-81$.

Rema, V., M. Armstrong-James, E.W. Jenkinson, and F.F. Ebner (1998) Short-term enriched experience potentiates whisker-pairing plasticity of barrel cortex neurons in adult rats. Society for Neuroscience Abstracts 24:(Abstract)

Rema, V., M. Armstrong-James, E.W. Jenkinson, and F.F. Ebner (1999) Short-term enriched experience potentiates whisker-pairing plasticity of barrel cortex neurons in adult rats. Somatosens.Mot.Res. 16:187(Abstract) 
Renehan, W.E. and B.L. Munger (1986) Degeneration and regeneration of peripheral nerve in the rat trigeminal system. I. Identification and characterization of the multiple afferent innervation of the mystacial vibrissae. J.Comp.Neurol. 246:12945.

Rhoades, R.W. (1981) Organization of somatosensory input to the deep collicular laminae in hamster. Behav.Brain Res. 3:201-222.

Rhoades, R.W., G.R. Belford, and H.P. Killackey (1987) Receptive-field properties of rat ventral posterior medial neurons before and after selective kainic acid lesions of the trigeminal brain stem complex. Journal of Neurophysiology 57:1577-600.

Rice, F.L., A. Mance, and B.L. Munger (1986) A comparative light microscopic analysis of the sensory innervation of the mystacial pad. I. Innervation of vibrissal folliclesinus complexes. J.Comp.Neurol. 252:154-74.

Richardson, F. (1909) A study of sensory control in the rat. Psychological Review 7:1-124.

Richter, C.P. (1957) On the phenomenon of sudden death in animals and man. Psychosomatic Medicine 19:191-198.

Robinson, D.L., M.E. Goldberg, and G.B. Stanton (1978) Parietal association cortex in the primate: sensory mechanisms and behavioral modulations. J.Neurophysiol. 41:910-32.

Rose, M. (1912) Histologische Lokalisation der Grosshirnrinde bei kleinen Säugetieren (Rodentia, Insectivoria, Chiroptera). J.Psychol.Neurol., Lpz. 19:389-479.

Rosina, A., L. Provini, M. Bentivoglio, and H.G. Kuypers (1980) Ponto-neocerebellar axonal branching as revealed by double fluorescent retrograde labeling technique. Brain Res. 195:461-466.

Russell, J.T. (1932) Depth discrimination in the rat. Journal of Genetic Psychology 167:136-159.

Sachdev, R.N.S., E.W. Jenkinson, and F.F. Ebner (1998) Response properties of barrel field neurons in the awake behaving rat. Society for Neuroscience Abstracts 24:(Abstract) 
Sachdev, R.N.S., E.W. Jenkinson, P. Melzer, and F.F. Ebner (1999) Dual electrode recording from the awake rat S1 barrel cortex. Somatosens.Mot.Res. 16:165166.(Abstract)

Sakata, H., H. Shibutani, and K. Kawano (1983) Functional properties of visual tracking neurons in posterior parietal association cortex of the monkey. J.Neurophysiol. 49:1364-80.

Savaki, H.E., C. Kennedy, L. Sokoloff, and M. Mishkin (1993) Visually guided guided reaching with the forelimb contralateral to a "blind hemisphere: a metabolic mapping study in monkeys. J.Neurosci. 13:2772-2789.

Savaki, H.E., C. Kennedy, L. Sokoloff, and M. Mishkin (1996) Visually guided guided reaching with the forelimb contralateral to a "blind hemisphere in the monkey: contribution of the cerebellum. Neuroscience 75:143-159.

Schäfer, E.A. (1898) J.Physiol.(Lond.) 23:310

Schiffman, H.R., R. Lore, J. Passafiume, and R. Neeb (1970) Role of vibrissae for depth perception in the rat (Rattus norvegicus). Anim Behav 18:290-2.

Schneider, G.E. (1967) Contrasting visuomotor functions of tectum and cortex in the golden hamster. Psychol.Forsch. 31:52-62.

Schneider, G.E. (1969) Two visual systems. Science 163:895-902.

Shambes, G.M., J.M. Gibson, and W. Welker (1978) Fractured somatotopy in granule cell tactile areas of rat cerebellar hemispheres revealed by micromapping. Brain Behav.Evol. 15:94-140.

Shammah-Lagnado, S.J., N. Negrao, and J.A. Ricardo (1985) Afferent connections of the zona incerta: a horseradish peroxidase study in the rat. Neuroscience 15:109-134.

Shipley, M.T. (1974) Response characteristics of single units in the rat's trigeminal nuclei to vibrissa displacements. J.Neurophysiol. 37:73-90.

Shosaku, A. (1985) A comparison of receptive field properties of vibrissa neurons between the rat thalamic reticular and ventro-basal nuclei. Brain Res. 347:36-40. 
Simons, D.J. (1978) Response properties of vibrissa units in rat SI somatosensory neocortex. J Neurophysiol 41:798-820.

Simons, D.J. and G.E. Carvell (1989) Thalamocortical response transformation in the rat vibrissa/barrel system. J Neurophysiol 61:311-30.

Simons, D.J., G.E. Carvell, A.E. Hershey, and D.P. Bryant (1992) Responses of barrel cortex neurons in awake rats and effects of urethane anesthesia. Exp.Brain Res. 91:25972.

Singer, W., H. Höllander, and H. Vanegas (1977) Decreased peroxidase labelling of the lateral geniculate neurons following deafferentation. Brain Res. 120:133-137.

Smith, R.L. (1973) The ascending fiber projections from the principal sensory trigeminal nucleus in the rat. J.Comp.Neurol. 148:423-45.

Sobel, E.C. (1990) The locust's use of motion parallax to measure distance. J.Comp.Physiol.[A]. 167:579-88.

Sperry, R.W. (1958) Anat.Rec. 131:297

Staines, W.A., H. Kimura, H.C. Fibiger, and E.G. McGeer (1980) Peroxidase-labelled lectin as a neuroaatomical tracer: evaluation in a CNS pathway. Brain Res. 197:490

Stanton, G.B. (1980) Topographical organization of ascending cerebellar projections from the dentate and interposed nuclei in Macaca mulatta: an anterograde degeneration study. J.Comp.Neurol. 190:699-731.

Stein, J.F. and M. Glickstein (1992) Role of the cerebellum in visual guidance of movement. Physiol Rev 72:967-1017.

Steindler, D.A. (1977) Trigeminocerebellar projections in normal and reeler mutant mice. Neurosci.Lett. 6:293-300.

Stöckel, K., M. Dumas, and H. Thoenen (1978) Uptake and subsequent retrograde axonal transport of nerve growth factor (NGF) are not influenced by neuronal activity. Neurosci.Lett. 61-64. 
Strick, P.L. (1985) How do the basal ganglia and cerebellum gain access to the cortical motor areas? Behav.Brain Res. 18:107-23.

Sugimoto, T., N. Mizuno, and K. Uchida (1982) Distribution of cerebellar fiber terminals in the midbrain visuomotor areas: an autoradiographic study in the cat. Brain Res. 238:353-370.

Sugitani, M., J. Yano, T. Sugai, and H. Ooyama (1990) Somatotopic organization and columnar structure of vibrissae representation in the rat ventrobasal complex. Exp.Brain Res. 81:346-52.

Sutherland, N.S. , and N.S. Mackintosh (1971) Animal Discrimination Learning. London: Academic Press.

Suzuki, D.A. and E.L. Keller (1984) Visual signals in the dorsolateral pontine nucleus of the alert monkey: their relationship to smooth-pursuit eye movements. Exp.Brain Res. 53:473-8.

Szel, A. and P. Rohlich (1992) Two cone types of rat retina detected by anti-visual pigment antibodies. Exp.Eye Res. 55:47-52.

Tello, J.F. (1923) Genese des terminaaisons motrices et sensitives. II. Terminaisons dans les poils de la souris blanche. Trav.Lab.Rech.Biol.Univ.Madrid 21:257-384.

Thier, P., W. Koehler, and U.W. Buettner (1988) Neuronal activity in the dorsolateral pontine nucleus of the alert monkey modulated by visual stimuli and eye movements. Exp.Brain Res. 70:496-512.

Thomas, H.M. (1910) Decussation of the pyramids - an historical inquiry. Johns Hopk.Hosp.Bull. $21: 304-311$.

Thor, D.H. (1975) Total suppression of irritable aggression in rats by sensory deprivation. Psychol.Rep. 37:432-4.

Thor, D.H. and W.B. Ghiselli (1973) Prolonged suppression of irritable aggression in rats by facial anesthesia. Psychol.Rep. 33:815-20.

Tiao, Y.C. and C. Blakemore (1976) Functional organization in the superior colliculus of the golden hamster. J.Comp.Neurol. 168:483-503. 
Ugolini, G. and H.G. Kuypers (1986) Collaterals of corticospinal and pyramidal fibres to the pontine grey demonstrated by a new application of the fluorescent fibre labelling technique. Brain Res. 365:211-227.

Ungerleider, L.G. and B.A. Brody (1977) Extrapersonal spatial orientation: the role of posterior parietal, anterior frontal, and inferotemporal cortex. Exp.Neurol. 56:265-80.

Ungerleider, L.G. , and M. Mishkin (1982) Two cortical visual systems. In D.G. Ingle, M.A. Goodale, and R.J.W. Mansfield (eds): Analysis of Visual Behaviour. Cambridge, MA.: MIT Press, pp. 459-486.

Van der Loos, H. (1976) Barreloids in mouse somatosensory thalamus. Neurosci.Lett. 2:16.

Van der Loos, H., J. Dorfl, and E. Welker (1984) Variation in pattern of mystacial vibrissae in mice. A quantitative study of ICR stock and several inbred strains. J Hered $75: 326-36$.

Van der Loos, H. and T.A. Woolsey (1973) Somatosensory cortex: structural alterations following early injury to sense organs. Science 179:395-398.

Veenman, C.L., A. Reiner, and M.G. Honig (1992) Biotinylated dextran amine as an anterograde tracer for single- and double-labeling studies. J.Neurosci.Methods $41: 239-254$.

Verhaart, W.J.C. and K. Mechelse (1954) The pedunculus cerebri and the capsula interna. Monatschr.Psychiat.Neurol. 127:65-88.

Vincent, S.B. (1912) The Function Of The Vibrissae of The White Rat. Behavior Monographs 1:1-86.

Vincent, S.B. (1913) The Tactile Hair Of The White Rat. J.Comp.Neurol. 23:1-36.

von Koranyi, A. (1890) J.Comp.Physiol.Psych. 53:322-327.

Waite, P.M. (1973) The responses of cells in the rat thalamus to mechanical movements of the whiskers. J.Physiol.(Lond.) 228:541-61. 
Waite, P.M. and B.G. Cragg (1982) The peripheral and central changes resulting from cutting or crushing the afferent nerve supply to the whiskers. Proc.R.Soc.Lond.B.Biol.Sci. 214:191-211.

Walker, E.P. (1968) Mammals of the world. Baltimore: Johns Hopkins.

Wall, G.L. (1934) The visual cells of the white rat. J Comp.Psychol. 18:363-366.(Abstract)

Wallace, G.K. (1959) Visual scanning in the dessert locust Schistocerca gregaria (Forskål). J.Exp.Biol 36:512-525.

Watson, C.R.R. and R.C. Switzer (1978) Trigeminal projections to cerebellar tactile areas in the rat - Origin mainly from $n$. interpolaris and n.principalis. Neurosci.Lett. 10:77-82.

Watson, J.B. (1907) Kinaesthetic And Organic Sensations. Psychological Review Monograph Supplement 8:75-75.

Welker, C. (1971) Microelectrode delineation of fine grain somatotopic organization of (Sml) cerebral neocortex in albino rat. Brain Res. 26:259-75.

Welker, C. (1976) Receptive fields of barrels in the somatosensory neocortex of the rat. J.Comp.Neurol. 166:173-89.

Welker, C. and T.A. Woolsey (1974) Structure of layer IV in the somatosensory neocortex of the rat: description and comparison with the mouse. J.Comp.Neurol. 158:43753.

Welker, E., P.V. Hoogland, and H. Van der Loos (1988) Organization of feedback and feedforward projections of the barrel cortex: a PHA-L study in the mouse. Exp.Brain Res. 73:411-35.

Welker, W.I. (1959) Escape, exploratory, and food-seeking responses of rats in a novel situation. J.Comp.Physiol.Psych. 52:106-111.

Welker, W.I. (1964) Analysis of sniffing of the albino rat. Behaviour 22:223-244. 
Westby, G.W., K.A. Keay, P. Redgrave, P. Dean, and M. Bannister (1990) Output pathways from the rat superior colliculus mediating approach and avoidance have different sensory properties. Exp.Brain Res. 81:626-38.

White, E.L. (1976) Ultrastructure and synaptic contacts in barrels of mouse SI cortex. Brain Res. 105:229-51.

White, E.L. and R.A. DeAmicis (1977) Afferent and efferent projections of the region in mouse SmL cortex which contains the posteromedial barrel subfield. J.Comp.Neurol. 175:455-82.

White, E.L. and A. Peters (1993) Cortical modules in the posteromedial barrel subfield (Sml) of the mouse. J.Comp.Neurol. 334:86-96.

Wiesendanger, R. and M. Wiesendanger (1982a) The corticopontine system in the rat. I. Mapping of corticopontine neurons. J.Comp.Neurol. 208:215-226.

Wiesendanger, R. and M. Wiesendanger (1982b) The corticopontine system in the rat. II. The projection pattern. J.Comp.Neurol. 208:227-238.

Wiesendanger, R., M. Wiesendanger, and D.G. Ruegg (1979) An anatomical investigation of the corticopontaine projection in the primate (Macaca fascicularis and Saimiri sciureus)--1l. The projection from frontal and parental association areas. Neuroscience 4:747-765.

Williams, M.N. and R.L. Faull (1987) The distribution and morphology of identified thalamocortical projection neurons and glial cells with reference to the question of interneurons in the ventrolateral nucleus of the rat thalamus. Neuroscience 21:767-80.

Wineski, L.E. (1983) Movements of the cranial vibrissae in the Golden hamster (Mesocricetus auratus). J.Zool.,Lond. 200:261-280.

Wineski, L.E. (1985) Facial morphology and vibrissal movement in the golden hamster. J Morphol 183:199-217.

Wise, S.P. and E.G. Jones (1977a) Cells of origin and terminal distribution of descending projections of the rat somatic sensory cortex. J.Comp.Neurol. 175:129-57. 
Wise, S.P. and E.G. Jones (1977b) Somatotopic and columnar organization of the corticotectal projection of the rat somatic sensory cortex. Brain Res. 129:

Wise, S.P. and E.G. Jones (1978) Developmental studies of thalamocortical and commissural connections in the rat somatic sensory cortex. J.Comp.Neurol. 178:187-208.

Wong-Riley, M.T. (1989) Cytochrome oxidase: an endogenous metabolic marker for neuronal activity. Trends Neurosci 12:94-101.

Wong-Riley, M.T.T. and C. Welt (1980) Histochemical changes in cytochrome oxidase of cortical barrels after vibrissal removal in neonatal and adult mice. Proc.Natl.Acad.Sci.USA 77:2333-2337.

Woolsey, C.N. (1947) Patterns of sensory representation in the cerebral cortex. Federation Proceedings 6:437-441.

Woolsey, C.N. (1952) Patterns of localisation in sensory and motor areas of the cerebral cortex. In AnonymousMilbank Memorial Fund: The biology of mental health and disease. New York: P. B. Hoeber,

Woolsey, C.N. (1958) Organisation of somatic sensory and motor areas of the cerebral cortex. In H.F. Harlow and C.N. Woolsey (eds): Biological and Biochemical Basis of Behavior. Madison: University of Wisconsin Press, pp. 63-82.

Woolsey, T.A., M.L. Dierker, and D.F. Wann (1975) Mouse Sml cortex: qualative, quantitive classification of Golgi-impregnated barrel neurons. Proc.Natl.Acad.Sci.USA 72:2165-2169.

Woolsey, T.A. and H. Van der Loos (1970) The structural organization of layer IV in the somatosensory region (SI) of mouse cerebral cortex. The description of a cortical field composed of discrete cytoarchitectonic units. Brain Res. 17:205-42.

Woolsey, T.A., C. Welker, and R.H. Schwartz (1975) Comparative anatomical studies of the SmL face cortex with special reference to the occurrence of "barrels" in layer IV. J.Comp.Neurol. 164:79-94. 
Woolston, D.C., J.R. La Londe, and J.M. Gibson (1982) Comparison of response properties of cerebellar- and thalamic- projecting interpolaris neurons. J Neurophysiol 48:160-73.

Woolston, D.C., J.R. La Londe, and J.M. Gibson (1983) Corticofugal influences in the rat on responses of neurons in the trigeminal nucleus interpolaris to mechanical stimulation. Neurosci.Lett. 36:43-48.

Wouterlood, F.G. and B. Jorritsma-Byham (1993) The anterograde neuroanatomical tracer biotinylated dextran- amine: comparison with the tracer Phaseolus vulgaris- leucoagglutinin in preparations for electron microscopy. J.Neurosci.Methods 48:75-87.

Yang, X., F. Hyder, and R.G. Shulman (1996) Activation of single whisker barrel in rat brain localized by functional magnetic resonance imaging. Proc.Natl.Acad.Sci.USA 93:475-478.

Yoshida, A., J.O. Dostrovsky, B.J. Sessle, and C.Y. Chiang (1991) Trigeminal projections to the nucleus submedius of the thalamus in the rat. J.Comp.Neurol. 307:609-25.

Yoshida, A., B.J. Sessle, J.O. Dostrovsky, and C.Y. Chiang (1992) Trigeminal and dorsal column nuclei projections to the anterior pretectal nucleus in the rat. Brain Res. 590:81-94.

Yuan, B., T.J. Morrow, and K.L. Casey (1985) Responsiveness of ventrobasal thalamic neurons after suppression of $S 1$ cortex in the anesthetized rat. J.Neurosci. 5:29718.

Zeki, S.M. (1974) Functional organization of a visual area in the posterior bank of the superior temporal sulcus of the rhesus monkey. J.Physiol.(Lond) 236:549-73.

Zeki, S.M. (1978) Functional specialisation in the visual cortex of the rhesus monkey. Nature 274:423-8.

Zeki, S.M. (1980) The responses of cells in the anterior bank of the superior temporal sulcus in macaque monkeys. J.Physiol.(Lond.) 308:85 
Zucker, E. and W.I. Welker (1969) Coding of somatic sensory input by vibrissae neurons in the rat's trigeminal ganglion. Brain Res. 12:138-56. 